\title{
Spatio-Temporal Dynamics of Pattern Formation in the Cerebral Cortex:
}

\author{
Visual Maps, Population Response and \\ Action Potential Generation
}

\section{PhD Thesis}

\author{
in partial fulfilment of the requirements \\ for the degree "Doctor of Philosophy $(\mathrm{PhD}) /$ Dr. rer. nat" \\ in the Neuroscience Program \\ at the Georg August University Göttingen, \\ Faculty of Biology
}

submitted by

Min Huang

born in

Fujian, P. R. China 
First Referee / Advisor:

Second Referee:

Third Referee:
Prof. Dr. Fred Wolf

Prof. Dr. Stefan Treue

Prof. Dr. Tobias Moser

Date of submission of the PhD thesis: March 16th, 2009

Date of thesis defence:

April 24th, 2009 
Herewith I declare, that I prepared the PhD Thesis

"Spatio-Temporal Dynamics of Pattern Formation in the Cerebral Cortex:

Visual Maps, Population Response and Action Potential Generation"

on my own and with no other sources and aids than quoted.

Göttingen, March 16th, 2009 
Dedicated to my little goose, for your dynamic growth 


\section{Contents}

Contents 3

List of Figures $\quad 5$

I Dynamics of Orientation_Preforence Maps in Feature Space Models of Visual Cortical Development 1

1 Introduction 3

1.1 Functional Architecture of the Mammalian Cerebral Cortex . . . . 3

1.2 Orientation Preference Maps . . . . . . . . . . . . . . . . . 3

1.3 Development and Plasticitv of OPMs . . . . . . . . . . . 5

1.4 How to Model Map Development . . . . . . . . . . . . . . . 5

1.4.1 Modeling the Collective Dvnamics of Cortical Plasticity . . 5

1.4.2 Pattern Formation as a Phase Transition . . . . . . . . 7

1.4.3 Time Scales of Map Development . . . . . . . . . . . . . 9

1.5 The Problem of Pinwheel Stability . . . . . . . . . . . . . 10

1.6 An Overview of mv Work . . . . . . . . . . . . . . 12

2 Analvsis of the Kohonen Model 15

2.1 Model Description . . . . . . . . . . . . . . . . . . . . 15

2.2 Linear Stability Analvsis . . . . . . . . . . . . . . . . . 17

3 Numerical Methods 23

3.1 Simulation Procedure . . . . . . . . . . . . . . . . . . 23

3.2 Choosing the Learning Rate . . . . . . . . . . . . . . . . . . 24

3.3 Principal Component Analvsis . . . . . . . . . . . . . . 25

3.4 Rotation of the Feature Coordinate Svstem . . . . . . . . . . 25

3.5 Map Analysis . . . . . . . . . . . . . . . . . . . . 25

4 Dvnamics of OPMs in 2D Models 29

4.1 Spontaneous Map Formation . . . . . . . . . . . . . . . . . . 29

4.2 Mans 'Frozen' bv a Small Set of Stimuli ... . . . . . . . . . . . . 31

4.3 Pinwheel Dvnamics Depends on Svstem Size . . . . . . . . . . . 34 
4.3 .1 Dvnamics in Small Svstems . . . . . . . . . . . . . 34

4.3 .2 Dvnamics in Large Svstems _. . . . . . . . . . . . . . 34

4.4 The Kinetics of Pinwheel Annihilation . . . . . . . . . . . . 37

4.5 Summary $\ldots \ldots \ldots \ldots \ldots$

5 Map Dynamics with Higher Dimensional Feature Spaces 41

5.1 Dimension Suppression Close to Svmmetrv-breaking Threshold . . 41

5.2 Pinwheel Annihilation for Low Dimensional Feature Spaces . . . . 44

5.3 Pinwheel Crvstallization for High Dimensional Feature Spaces . . 47

5.4 Summary . . . . . . . . . . . . . . . . . . . 47

6 Map Dynamics with Non-Gaussian Stimulus Distributions 51

6.1 Models with a Spherical Feature Space . . . . . . . . . . . . 51

6.2 Models with a Toroidal Feature Space . . . . . . . . . . . . . . 54

$\begin{array}{lll}7 & \text { Discussion and Conclusion of Part I } & 57\end{array}$

\begin{tabular}{ll}
\hline Bibliography & 63
\end{tabular}

II Action Potential Dynamics and Response Function of $\begin{array}{ll}\text { Cortical Neuron Models } & 69\end{array}$

$\begin{array}{lll}8 & \text { Introduction } & 71\end{array}$

8.1 Action Potential Dvnamics in Phase Plots . . . . . . . . . . . 71

8.2 Population Coding . . . . . . . . . . . . . . . . . . 73

8.3 Fast Onset Dvnamics of AP Initiation . . . . . . . . . . . 73

8.4 AP Initiation and Back-Propagation in Multi-Compartment Models 74

8.5 An Overview of mv Work . . . . . . . . . . . . . . 74

9 Basics of Dvnamic Response in Conductance Based Models $\quad 77$

9.1 Computational Models . . . . . . . . . . . . . . . . . . 77

9.1.1 Wang-Buzsaki Models of AP Generation . . . . . . . . 77

9.1.2 Modeling Background Noise in the Cortex . . . . . . . 78

9.2 Subthreshold Response to Noisv Inputs . . . . . . . . . . . . . 80

9.3 Firing-rate Response to Noisv Inputs . . . . . . . . . . . . . 84

9.3.1 Stationarv Response Function . . . . . . . . . . . . . 84

9.3.2 Frequencv Dependence of the Population Response . . . . 86

9.3.3 Fast Response Properties with Fast AP Onset? . . . . . . 87

Appendix: Channel Kinetics of CB models . . . . . . . . . . . . . 90

10 Dynamic Response in Models with Cooperative Channel Gating 91

10.1 Introduction . . . . . . . . . . . . . . . . . . 91

10.2 AP Generator with Channel Cooperativity . . . . . . . . . . . 93 
10.3 AP Waveforms and Onset Dvnamics . . . . . . . . . . . . . . . . 97

10.4 Impact on Dvnamic Response Properties . . . . . . . . . . . . . . 100

10.5 Summarv and Discussion . . . . . . . . . . . . . . . . . . 101

11 AP Propagation and Neuron Geometry 105

11.1 Introduction . . . . . . . . . . . . . . . . . . . . . 105

11.2 Methods . . . . . . . . . . . . . . . . 106

11.2 .1 Spatiotemporal Dvnamics . . . . . . . . . . . . 106

11.2.2 Model Neuron Geometry . . . . . . . . . . . . . . . . . 108

11.2 .3 Computational Methods . . . . . . . . . . . . . . 109

11.3 Simulation Results . . . . . . . . . . . . . . . . . . . . 110

11.4 Onset Rapidness vs. Propagation Velocity . . . . . . . . . . . . . 114

11.5 Effects of Lateral and Local Currents . . . . . . . . . . . . . . . . 116

11.6 Decomposing the Somatic AP . . . . . . . . . . . . . . . . . . . . 119

11.7 Effects of Delaved Sodium Channel Kinetics . . . . . . . . . . . . 121

11.8 Summary and Discussion . . . . . . . . . . . . . . . 126

12 Dynamics of AP Initiation in the Soma-Proximal Axon-Complex 129

12.1 Introduction . . . . . . . . . . . . . . . . . . . 129

12.2 Model Description

12.2 .1 Model Neuron Morphology . . . . . . . . . . . . . . . . . . 130

12.2.2 Passive Electrical Parameters . . . . . . . . . . . . . . 131

12.2.3 Channel Distribution . . . . . . . . . . . . . . . 132

12.3 Models with Active Dendrites . . . . . . . . . . . . . . . . . 133

12.3.1 Sites of AP Initiation . . . . . . . . . . . . . . . 134

12.3.2 Onset Dvnamics of the Somatic AP . . . . . . . . . . . 135

12.4 Models with Passive Dendrites . . . . . . . . . . . . . . . . . 136

12.4.1 Sites of AP Initiation . . . . . . . . . . . . . . . . 137

12.4.2 Onset Dvnamics of the Somatic AP . . . . . . . . . . . . . 138

12.5 Impact on Dvnamic Response Properties . . . . . . . . . . . . . . 139

12.5.1 Voltage Dvnamics at the Soma and the AIS . . . . . . . . 140

12.5.2 Frequency Response to Oscillatorv Noisv Inputs . . . . . . 140

12.6 Summary and Discussion . . . . . . . . . . . . . . . . . . . . 143

13 Discussion and Conclusion of Part II 145

\begin{tabular}{lr}
\hline Bibliography & 151
\end{tabular} 
\begin{tabular}{ll}
\hline Summary & 159
\end{tabular}

$\begin{array}{ll}\text { Acknowledgements } & 161\end{array}$

$\begin{array}{ll}\text { Curriculum Vitae } & 162\end{array}$ 


\section{List of Figures}

1.1 Spatial pattern of the OPMs .............. . . 4

1.2 OPM in rewired ferret auditory cortex . . . . . . . . . . 6

1.3 OPMs and cortical function on different spatial scales . . 8

1.4 Map dvnamics in one simulation of the Kohonen model . 11

1.5 Segregated ODCs and OPM in strabismic cat V1 . . . . 13

2.1 Coordinate transform . . . . . . . . . . . . . . . . . . 18

2.2 Representative spectra of pattern growth rates $\lambda(k)] \ldots 21$

4.1 Emergence of orientation preference . . . . . . . . . 30

4.2 The tuning strength of OPMs varies with size of CCDs . 31

4.3 Maps 'frozen' by a small set of stimuli . . . . . . . . . . . 32

4.4 Dvnamics varv with size of the presented stimulus set . . 33

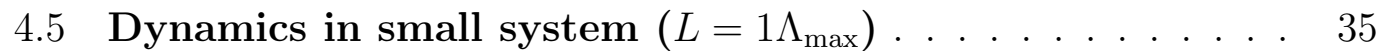

4.6 Svstem size dependence of OPM dvnamics . . . . . . . . 36

4.7 Mechanical analog: the svstem size effect . . . . . . . . . 37

4.8 Kinetics of pinwheel annihilation . . . . . . . . . . . . . . . . 39

5.1 Dimension suppression in small svstems (2D+3D) . . . . 42

5.2 Bifurcation diagrams (Gaussian distributions) . . . . . . . 43

5.3 Example maps of different feature dimensions $\left(\sigma=0.9 \sigma^{*}\right) \quad 45$

5.4 Pinwheel dvnamics $\left(2 \mathrm{D}+3 \mathrm{D}, \sigma=0.667 \sigma^{*}\right) \ldots \ldots \ldots \ldots$

5.5 Pinwheel dvnamics (2D $\left.+4 \mathrm{D}, \sigma=0.667 \sigma^{*}, L=4 \Lambda_{\mathrm{max}}\right)$. . . 48

5.6 Pinwheel crvstallization $\left(2 \mathrm{D}+4 \mathrm{D}, \sigma=0.667 \sigma^{*}\right) \ldots \ldots \ldots$

5.7 Pinwheel crystallization $\left(2 \mathrm{D}+5 \mathrm{D}, \sigma=0.667 \sigma^{*}\right) \ldots \ldots .49$

6.1 Bifurcation diagrams for the spherical model . . . . . . . . 52

6.2 Pinwheel dvnamics in $S^{2}$ feature space model . . . . . . . 53

6.3 Bifurcation diagrams for the toroidal models . . . . . . . . 54

6.4 Maps generated from toroidal feature spaces . . . . . . . 55

8.1 AP dynamics in neocortical neurons and in HH models . 72

9.1 Noisy input from presvnaptic neurons . . . . . . . . . . . . 79 
9.2 MP fluctuation and mean firing rates . . . . . . . . . . . . 85

9.3 Neuronal response to noisy sinusoidal inputs . . . . . . . . 86

9.4 Frequency modulation of population firing rate . . . . . . . 87

9.5 Fast response speed with fast AP onset . . . . . . . . . . . . 88

10.1 Channel clustering and cooperative gating in experiments 92

10.2 Fixed points of the cooperative activation variable . . . . . 94

10.3 Cooperative gating with varving degrees of coupling strength 96

10.4 AP dvnamics with a subpopulation of cooperative channels 98

10.5 Phase diagram of the AP waveforms . . . . . . . . . . 99

10.6 Threshold variability insensitive to channel cooperativity 100

10.7 Cooperative gating improves the high-frequency response 102

11.1 Voltage dynamics in a cable and in a point neuron . . . . 107

11.2 Morphology of the geometric model . . . . . . . . . . . . . . 108

11.3 Two simulation examples in the geometric model . . . . . 111

11.4 Parameter dependence of somatic AP onset rapidness . . 112

11.5 Phase diagrams of somatic AP onset rapidness . . . . . . . 113

11.6 AP onset rapidness and the propagation velocity . . . . . 115

11.7 The big AP in space and the lateral current. . . . . . . . . 117

11.8 AP waveforms affected by termination of current injection 118

11.9 Two components of the somatic action potential . . . . . 119

11.10 Contributions of lateral current and local sodium current 120

11.11 Sodium currents with delaved channel kinetics. . . . . . . . 123

$11.12 \mathrm{AP}$ generation in WB models with $\mathrm{m}^{1}$ and $\mathrm{m}^{3}$ kinetics . . 124

11.13 Somatic AP onset in $\mathrm{WB} \mathbf{m}^{\mathbf{1}}$ and $\mathrm{m}^{\mathbf{3}}$ models . . . . . . . 125

11.14 Somatic AP onset in the Mainen-Sejnowski model . . . . 126

12.1 Modeling the morphology of a mvelinated cortical neuron 130

12.2 Simulation of neurons with active dendrites . . . . . . . . 133

12.3 AP initiation sites in neurons with active dendrites . . . . 134

12.4 Somatic AP onset in neurons with active dendrite . . . . 135

12.5 Simulation example of neurons with passive dendrite . . . 136

12.6 AP initiation site in neurons with passive dendrites . . . 137

12.7 Somatic AP onset in neurons with passive dendrite . . . . 138

12.8 Phase diagram in models with passive dendrites . . . . . . 139

12.9 Response to noisv inputs in models with $\overline{\mathbf{g}}_{\mathrm{Na}} \times 10$ at AIS 141

12.10 Frequency modulation in multi-compartment models . . . 142 


\section{Part I}

\section{Dynamics of Orientation Preference Maps in Feature Space Models of Visual Cortical Development}





\section{Chapter 1}

\section{Introduction}

\subsection{Functional Architecture of the Mammalian Cere- bral Cortex}

The cerebral cortex is a key structure of our brain that plays an important role in cognitive functions such as perception, attention, thought and language. In the functional modules of cerebral cortex, so called cortical columns, neurons are arranged in a slab of tissue extending perpendicular to the cortical surface. Neurons within a column usually share similar functional properties. In a cortical layer parallel to the surface, neuronal selectivities vary systematically. Such two-dimensional(2D) patterns of functional maps have been discovered in many cortical areas, e.g., a map of our body surface found in somatosensory cortex, a map of sound frequency in the primary auditory cortex and a retinotopic map together with other functional maps in the primary visual cortex.

For our understanding of brain mappings, visual maps provide especially good examples not only because the in vivo cortical activities can be recorded along with precise control of visual stimuli, but also due to the rich patterns of multiple features they encode. Neurons in the primary visual cortex are selective to the complex structure of a natural scene, including visual field position, contour orientation, direction of motion, spatial frequency, and binocular visual cues such as ocular dominance. Most primates and carnivores show a highly organized spatial pattern of preferred stimulus features across the visual cortex (Hubel \& Wiesel, 1962; Frostig et al., 1990; Hubel, 1995; Welikv et al., 1996; Ohki et al., 2005). The spatial complexity of these visual maps challenges the theory of map formation to coordinate between different feature maps in one cortical area. 


\subsection{Orientation Preference Maps}

Orientation columns are among the most thoroughly investigated patterns of cortical functional architecture (Blasdel \& Salama. 1986: Bosking et al.. 1997: Bonhoeffer \& Grinvald. 1991: Crair et al., 1997al.b: Hübener et al.. 1997: Kaschube et al. 2000: Kaschube. 2005: Löwel et al.. 1987. 1998: Mooser et al., 2004: Müller et al., 2000; Ohki et al., 2006). Figure 1.11A shows a typical example of an orientation preference map (OPM) observed in the primary visual cortex of cat. The OPM is smooth almost everywhere in the sense that neighboring neurons normally prefer similar orientations. The continuous change of orientation selectivity is disrupted at local regions with a radial arrangement of iso-orientation domains around a common center. Such regions have been termed 'pinwheels' and are ubiquitous structural elements of the orientation map. Applying two-photon calcium imaging in vivo, a recent study (Ohki et al., 2006) has visualized that pinwheel centers are highly ordered down to the level of single cells (Figure 1.1B).
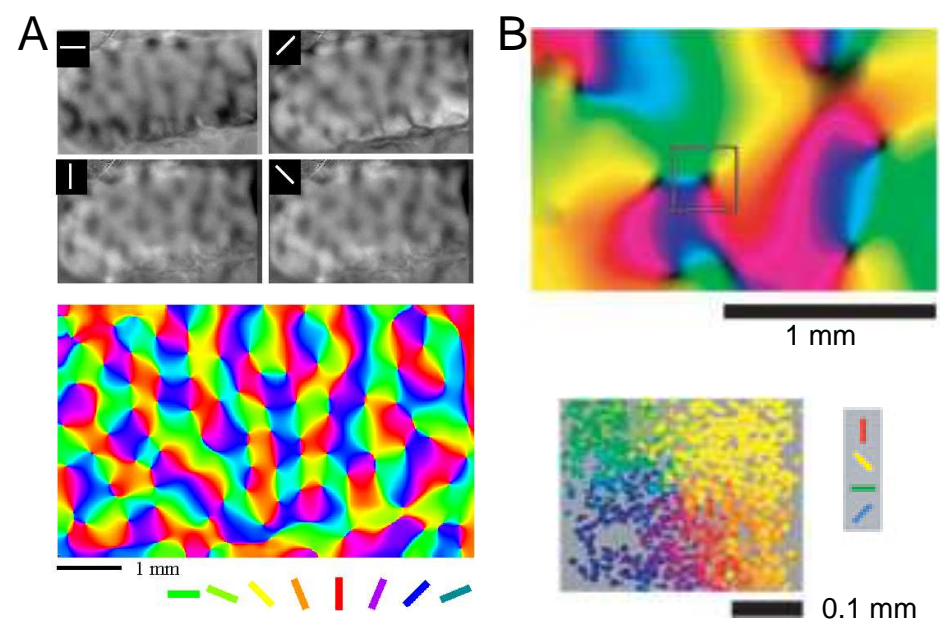

Figure 1.1: Spatial pattern of the OPMs (A) The map was recorded by optical imaging in a $7 \mathrm{~mm} \times 4 \mathrm{~mm}$ area of cat V1. Top: Cortical activity patterns respond to four stimulus angles $\left(0^{\circ}, 45^{\circ}, 90^{\circ}, 135^{\circ}\right.$, shown in inset of each panel) from one animal. Black areas of each panel indicate areas of cortex that were preferentially activated by a given stimulus, and light gray areas indicate areas that were active during presentation of the orthogonal angle. Bottom: OPM obtained by vector summation of data obtained for each angle. The colors represent different orientation preferences. Data provided by Dr. Kisvarday (University of Bochum). (B) Microstructure of orientation pinwheels. Top: OPM from optical imaging, recorded in area 18 of cat visual cortex. The square region indicates the typical structure of pinwheels. Bottom: Two-photon calcium imaging demonstrated the same pinwheel center with single cell resolution. Figure modified from Ohki et al. (2006). 
In the striate cortex of an adult cat, several hundreds of pinwheels connected by iso-orientation lines form a roughly repetitive pattern. The column spacing $\Lambda$ measures the average distance of adjacent iso-orientation domains, which is in the range of $1 \mathrm{~mm}$. A hypercolumn of size $\Lambda \times \Lambda$ can be considered as an information processing unit, which represents all kinds of orientations at a given visual field position.

\subsection{Development and Plasticity of OPMs}

How do such cortical maps emerge during development? The neural network involved in one cortical hypercolumn is extremely large. On average 100,000 neurons and a billion synapses are packed into $1 \mathrm{~mm}^{3}$ of cortex. This enormous number of neurons and connections makes it unlikely to completely specify neural connectivity patterns prenatally on the genetic level.

It has been a long-standing hypothesis that cortical maps emerge through a self-organizing process, which is to some extent driven by sensory experience. One of the most fascinating evidences in favor of this hypothesis comes from the cortex of so called rewired animals (Sharma et al., 2000). In ferrets, thalamocortical projections from the retina were experimentally redirected to drive the auditory pathway at a very early stage of development (see Figure 1.2). As a consequence similar patterns of OPMs have been recorded in the area that would normally be the primary auditory cortex. This finding indicates a general capability of pattern formation in the cerebral cortex to generate a sensory representation as found in V1 when merely given appropriate inputs.

To which extent visual experience might shape the development of OPMs in the normal cortex is yet to be uncovered. It seems that the appearance of crude patterns in V1 of primates at birth and in cats at the time of natural eye opening does not require structured visual experience (for a review see Sur \& Leamev (2001)). However, patterns of spontaneous activity have been recorded in the developing dorsal lateral geniculate nucleus (LGN) that may play an important role in the initial establishment of the cortical patterns. (Weliky \& Katz, 1999).

Moreover, visual inputs are essential in the next stage for the maintenance of the map dynamics. Long term deprivation of visual experience by binocular suture can cause a loss of orientation selectivity and degradation of the visual cortical maps (Crair et al., 1998). In addition, an influence of restricted visual experience has been reported in kittens reared in a striped environment consisting of one single orientation. Here the experienced orientation is over-represented in the cortical area up to twice compared to the orthogonal orientation (Sengpiel et al., 1999).

Taken together, these observations suggest that OPMs are subject to dynamical changes and susceptible to the visual experience. 

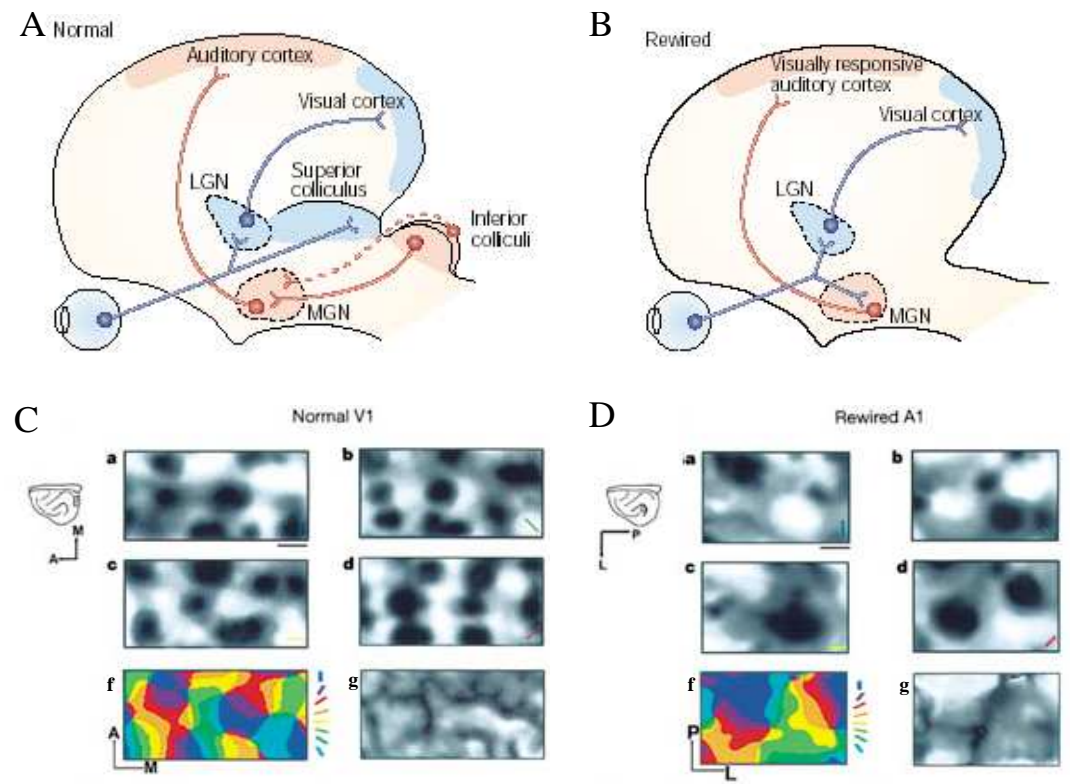

Figure 1.2: OPM in rewired ferret auditory cortex (A) In normal animals, the retina projects to the primary visual cortex via the lateral geniculate nucleus (LGN) and superior colliculus (blue pathway). The medial geniculate nucleus (MGN) innervating the primary auditory cortex receives most of its subcortical afferents from the ipsilateral inferior colliculus (red line) and from the contralateral inferior colliculus (red dashed line). (B) In rewired animals, extensive neonatal deafferentation of the MGN induces the retino-MGN projections to innervate the primary auditory cortex. This effect can be obtained by destroying the inferior colliculus unilaterally along with a lesion of the superior colliculus which carries inputs from the contralateral inferior colliculus. (C, D) Orientation maps in normal V1 and 'rewired A1'; scale bar: $1 \mathrm{~mm}$. (a-d) Cortical activity patterns evoked by vertical, left oblique, horizontal and right oblique grating stimuli, respectively. (f) Composite map of orientation preference. Color bar, key for representing orientations. (g) Map of orientation vector magnitude. Dark regions indicate low magnitude. Modified from Sharma et al. (2000): Sur \& Leamey (2001). 


\subsection{How to Model Map Development}

\subsubsection{Modeling the Collective Dynamics of Cortical Plas- ticity}

Cortical maps reflect computations operating in relatively large scale networks. As revealed by single-orientation maps (Figure [1.3A), cortical neurons are active in local groups, called co-activated cortical domains (CCDs) in the following. Each domain is composed of tens of thousands of neurons. These neurons not only receive feed forward inputs but also extensive synaptic input from intra-cortical connections. Such connections are predominantly found in domains with similar orientation preference (Figure 1.3B ). Hence the competitive forms of Hebbian learning (Hebb, 1949) of synaptic plasticity ('Neurons that fire together wire together') are often used to model cortical map development. One of the paradigm models for the activity-dependent map formation is Kohonen's self-organizing feature map model.

The Kohonen model describes the development of cortical maps on a mesoscopic level (Kohonen, 1982, 1993; Obermaver et al., 1990, 1992). The cortical network is described by formal units representing hundreds of neurons. Neurons within each unit share a common profile of selectivity such that competitive Hebbian learning is performed on the level of these units. This mesoscopic level of description improves the computational efficiency of simulating large scale networks. Numerical simulations of this model have shown that orientation prefer-

ence maps can develop from an initial unselective state (Obermaver et al., 1990; Swindale, 2000, 2004).

\subsubsection{Pattern Formation as a Phase Transition}

How does a spatial pattern emerge from an initial homogenous state and how should this process be characterized? Pattern formation as spontaneous symmetry breaking has been studied in many physical systems undergoing e.g. liquid-gas transitions or a ferromagnetic phase transition (Manneville. 1990: Cross \& Hohenberg, 1993). In these systems, a new organization emerges at a critical value of certain control parameters like the critical temperature.

A collective behavior mimicking phase transitions is expected in visual map development given that the cortical activity patterns are strongly shaped by intracortical interactions (Ritter \& Schulten. 1988: Miller et al.. 1989: Obermayer et al., 1992; Wolf et al., 2000). The pattern formation of OPMs is controlled by the size $\sigma$ of co-activated cortical domains (CCDs). If the CCDs are extremely large so that the entire cortex is co-active, no segregation of OPMs can occur. A previous theoretical study (Wolf et al., 2000) suggested that the transition from such a homogenous state to the spontaneous formation of a map is not gradual but occurs at a discrete instability point with a critical size $\sigma^{*}$ of CCDs. At this 

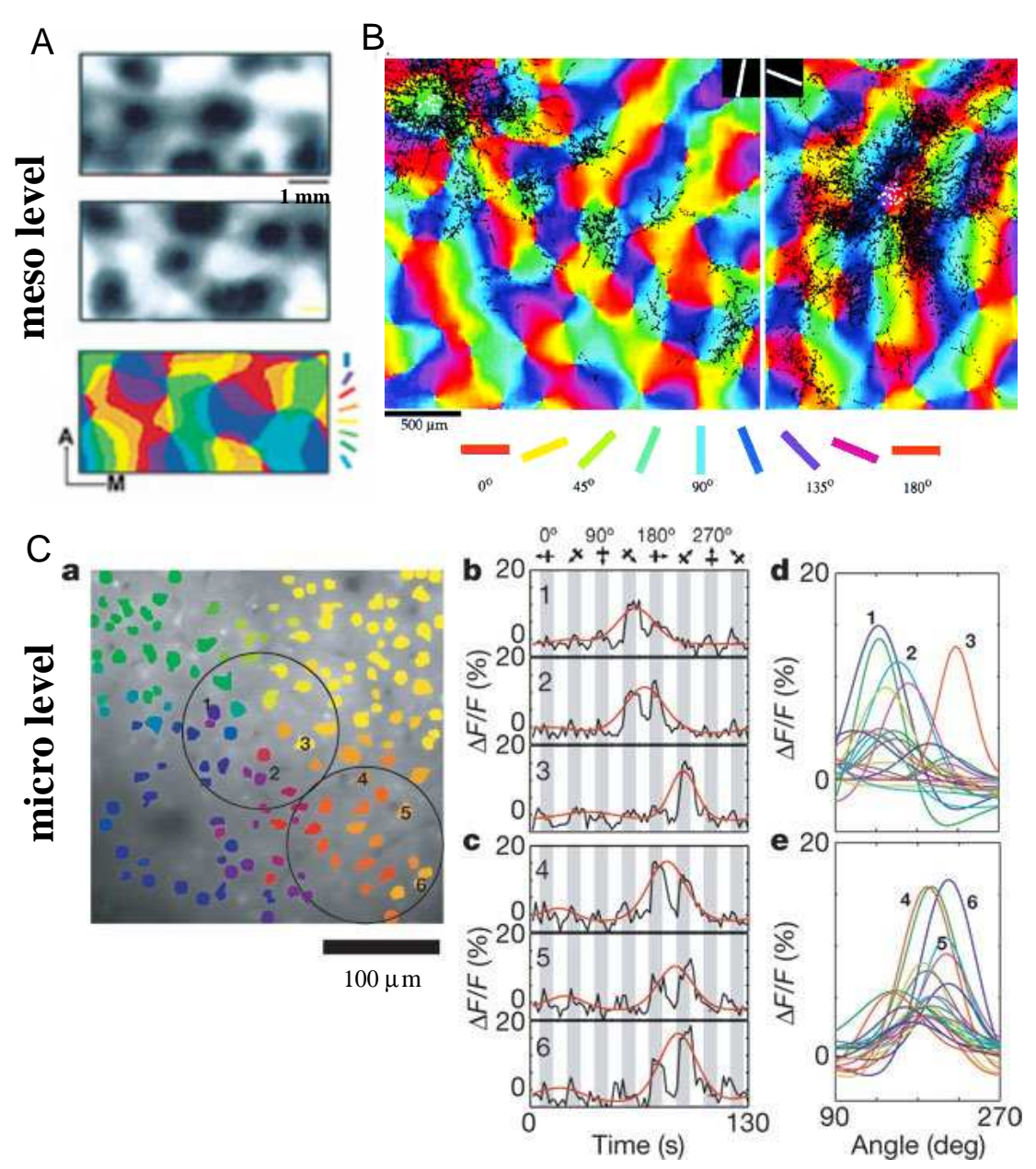

Figure 1.3: OPMs and cortical function in different spatial scales (A) The single condition maps for vertical (upper panel), for horizontal orientation (middle panel) and the composite OPM (bottom panel) recorded in ferret V1. Maps taken from Sharma et al. (2000). (B) The horizontal connections in two maps of tree shrew striate cortex. White dots: Biocytin taken up by cells at injection site; Black dots: Labeled boutons found in the neighborhood of injection site and in the domains of similar orientation preference. Taken from Bosking et al. (1997). (C) The highly ordered orientation preference of cells near a pinwheel center recorded in cat V2 by two-photon microscopy and tuning curves of individual neurons. Taken from Ohki et al. (2006). 
point of instability, a small deviation of $\sigma$ (less than 1\%) dramatically change the behavior of the system. If we consider the synaptic connections of neurons in a locally co-activated group, one percent of difference in the interaction range is not expected to affect the properties of individual neurons significantly. The phase transition that leads to the emergence of orientation selectivity is thus one of the collective behaviors that can hardly be anticipated from the study of cellular and subcellular processes.

In the theory of pattern formation, systems close to threshold or far from threshold are known to behave quite differently (Cross \& Hohenberg, 1993). Close to threshold systems deal with small deviations from a stationary homogeneous state, which breaks down at the instability point with a certain growth rate of the emerging pattern. Systems far from threshold lie in so-called highly nonlinear regimes. Their temporal evolution involves extensive pattern rearrangement to match different parts of the system. However, little attention has been dedicated to distinguishing such different dynamical regimes in modeling map development.

Previous studies often chose a small size $\sigma$ of co-activated cortical domains (CCDs), about one order of magnitude lower than the pattern wavelength (Goodhill \& Cimponeriu, 2000; Swindale, 2000, 2004; Yu et al.,, 2005; Farlev et al., 2007). In some of these studies (e.g., Swindale (2004); Goodhill \& Cimponeriu (2000)), the size $\sigma$ of co-activated cortical domains (CCDs) was continually reduced in simulations to facilitate map convergence, a process referred to as annealing.

What is the realistic size of CCDs in the activity patterns of OPMs? As illustrated in Figure 1.3A, the size of co-activated domains in single condition maps is similar to that of the iso-orientation domains in the OPM, a value of the same order of magnitude as the pattern wavelength. An independent measure to estimate the value of $\sigma$ is based on the tuning width of individual neurons (Figure $1.3 \mathrm{C}$ ), which covers an orientation difference of about $90^{\circ}$. In other words, half of each hypercolumn is activated by a single orientation given the smoothness of the map. These observations thus question the use of very small sized CCDs in simulations. They demand to carefully characterize the dynamics of developmental models in particular in the regime of larger CCD sizes.

Our work aims to provide a quantitative study on the cortical map development in different dynamical regimes with respect to the critical threshold of pattern formation. For this purpose, we first performed a stability analysis around the homogeneous state to reveal the critical point of pattern formation and the growth rate of the emerging pattern.

\subsubsection{Time Scales of Map Development}

Spatially extended dynamical systems often exhibit processes occurring on various time scales. This fact reflects that in a spatially extended system, subsystems separated by a large spectrum of distances need to coordinate their behavior, which proceeds on larger time scales for more distant subsystems. Are such 
long time scales important for map development in the visual cortex? Taking the primary visual cortex of a cat as an example, selective activity patterns are established within a few days between the second and the third postnatal week, which is followed by a critical period of map plasticity and refinement lasting for three months in kittens (for a review see Sur \& Leamev (2001)). The minimum duration of strong plasticity is thus at least one or two orders of magnitude larger than the initial period needed for establishment of a first map.

Numerical simulations have shown the structure of visual maps developed in an activity-dependent process of synaptic plasticity (Durbin \& Mitchison, 1990; Ferster \& Miller. 2000: Miller. 1992. 1994: Ferster \& Miller. 2000: CarreiraPerpiñán et al., 2005; Swindale, 2000, 2004; Yu et al., 2005; Farlev et al., 2007). However, most of these studies have treated the emerging pattern as a static layout with little concern on the time scales relevant for cortical development.

To appropriately resolve the temporal dynamics in a developmental model, we analytically derived the intrinsic time scale of the emergence of a first map. Each step of synaptic learning was then performed on a much smaller time scale compared with this intrinsic time scale of map development and the dynamics of the entire system was tracked in simulations for a period much longer than the intrinsic time scale.

\subsection{The Problem of Pinwheel Stability}

Modeling the dynamics of OPMs is central for understanding the pattern of pinwheels in the map. On the microscopic level, a recent study using two-photon calcium imaging (Ohki et al., 2006) has revealed a highly ordered arrangement of neurons at pinwheel centers. A pinwheel is not a functional defect with unresponsive neurons but rather consist of neurons selective for all different orientations (Figure $1.3 \mathrm{C}$ :a). Orientation tuning curves of individual neurons in the pinwheel center showed similar response strength and tuning width compared to those in the iso-orientation domain (Figure 1.3 C:b-e).

It is important to note that such pinwheels do not invariably occur in models of cortical map development. Simulations of various models have shown that an initially pinwheel-rich state can decay towards a pinwheel-sparse pattern of orientation stripes. a process known as spontaneous pinwheel annihilation (Wolf

\& Geisel, 1998; Koulakov \& Chklovskii, 2001; Lee et al., 2003; Cho \& Kim, 2004).

To illustrate this problem, we performed a simulation of the Kohonen model applying the parameters used in Farlev et al. (2007) but presenting the system with a 20-fold number of stimuli (30 million versus 1.5 million) compared to the original study. As shown in Figure 1.4 the dynamics of the OPM appeared to be unstable, where the pinwheel numbers reduced continually as more and more stimuli were presented to train the model cortex (Figure 1.4B). Simultaneously, the column spacing increased over time so that the size of iso-orientation 
domains expanded (Figure 1.4C). Multiplication of these two measures yields a dimensionless quantity, the scaled pinwheel density $\rho \Lambda^{2}$, which represents the average number of pinwheels in one hypercolumn (Figure 1.4D). None of the spatial characteristics of the OPM had become stationary (Figure 1.4 B-D, dashed line) for the results reported in the studv bv Farlev et al. (2007). Intriguinglv. Farley et al. (2007) did not mention and appeared unaware of this dramatic dynamical rearrangement.
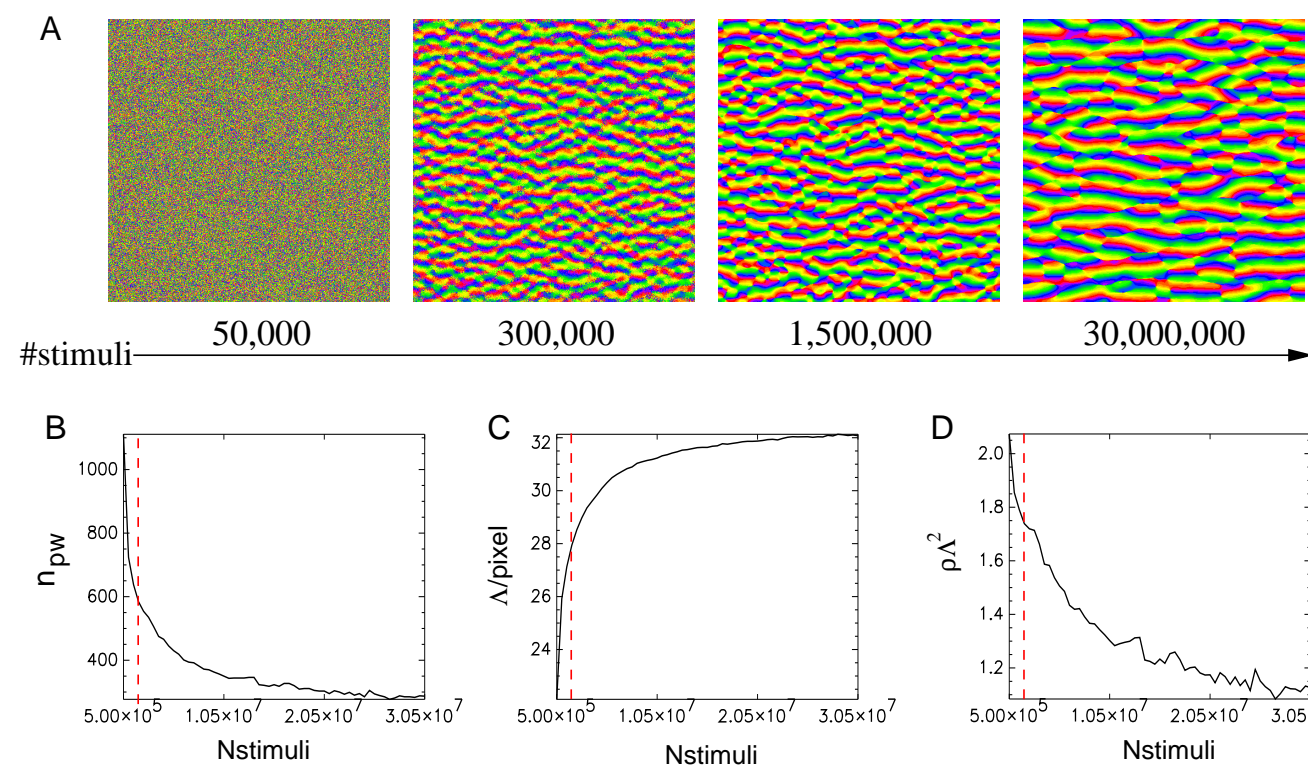

300,000

$1,500,000$

$30,000,000$
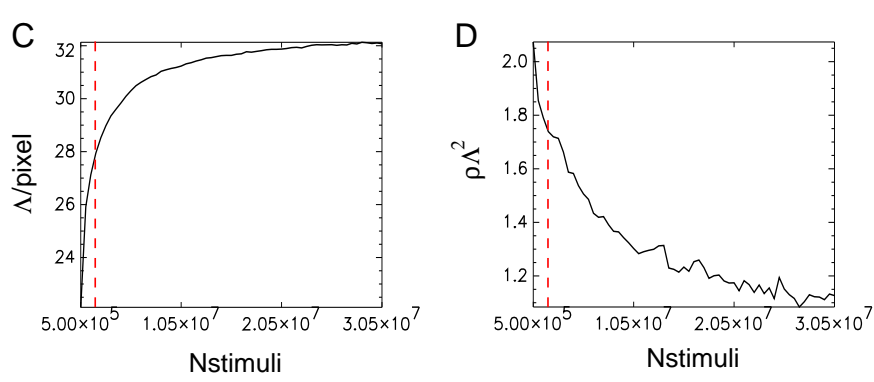

Figure 1.4: Map dynamics in one simulation of the Kohonen model (A) The patterns of OPMs evolved in one simulation as the number of presented stimuli increases. (B) The number of pinwheels decreased during the simulation. (C) Increase of the average column spacing. (D) The scaled pinwheel density $\rho \Lambda^{2}$ decreased to a value much lower than 2. Dash lines: the stage of the simulation at the same amount of stimuli presented in Farley et al. (2007).

One possible explanation for the persistence of pinwheels in the visual cortex might be that, after the initial establishment of an early pinwheel-rich pattern, the dynamical development of OPMs is terminated as the 'critical period' comes to an end which is about the first couple of months after birth (Katz \& Crowlev, 2002). However, there is no direct evidence of molecules or genes that terminate this dynamical process. In contrast, a stimulus induced non-local reorganization of the maps has been reported even in adult cats (Godde et al., 2002). This indicates that map plasticity is maintained in the adult. Even if OPM plasticity is restricted to the critical period, this period lasts many months and peaks only four weeks after an OPM is initially established (Sur \& Leamev, 2001). As the initial establishment of an OPM requires only a period of a few days (Chapman 
et al., 1996), realistic simulations of map development should cover time scales much longer than that needed for the initial map formation.

\section{Two Hypotheses}

From a theoretical perspective, two hypotheses have been proposed to solve the problem of pinwheel stability. (1) Pinwheels are stabilized by interactions of different features under the constraint of coverage optimization (Wolf \& Geisel, 1998). (2) The spatial layout of OPM is shaped by intrinsic processes such as long-range intracortical interactions (Wolf, 2005).

The first hypothesis is based on the observed spatial relationships between different feature maps in the visual cortex (Crair et al., 1997b a; Hübener et al., 1997; Löwel et al., 1998; Müller et al., 2000). Previous studies revealed a tendency for pinwheel center singularities of OPMs to lie in the center regions of the ocular dominance columns(ODCs), and for iso-orientation bands to cross ocular dominance borders at right angles (Figure [1.5). A general framework of dimension reduction (Durbin \& Mitchison, 1990) has been proposed for understanding cortical mappings that preserve neighborhood relations in the feature space. To avoid functionally 'blind spots' in the visual field, the concept of coverage uniformity constrains the maps to fill the input space with near-uniform density while maintaining continuity. This leads to maps where rapid changes in one feature component are correlated with slow changes in other components. Numerical studies further suggested that strong ocular dominance segregation can slow down the process of pinwheel annihilation in developmental models (Wolf \& Geisel, 1998).

However, recent quantitative studies (Kaschube, 2005; Kaschube et al., 2006) found a universal pinwheel density in adult animals of several species despite substantially different organizations of ocular dominance columns. This appears inconsistent with theoretical prediction that suggested a correlation between the numbers of pinwheels per hypercolumn and the degree of ocular dominance segregation in different species (for discussion see Wolf \& Geisel (1998)). This raises the question whether the OPMs are stabilized by interaction with other maps, or whether the ubiquitous pinwheel structure is selected by a universal pattern forming process independent of the number of mapped features.

\subsection{An Overview of my Work}

The objective of this project is to develop a methodology for analysis and simulations of cortical dynamics from the perspective of nonlinear dynamical systems and applying this method to comprehensively study the problem of pinwheel stability in models of interacting columnar patterns. The model behavior was characterized in different dynamical regimes, for various system sizes, feature 

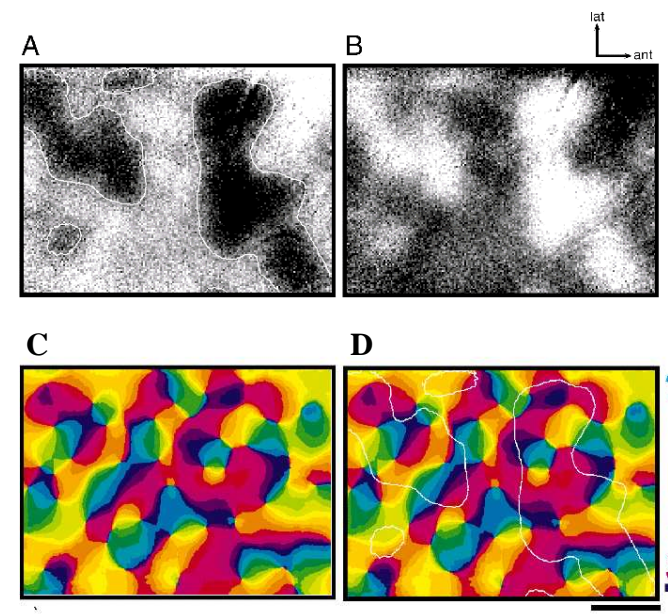

D

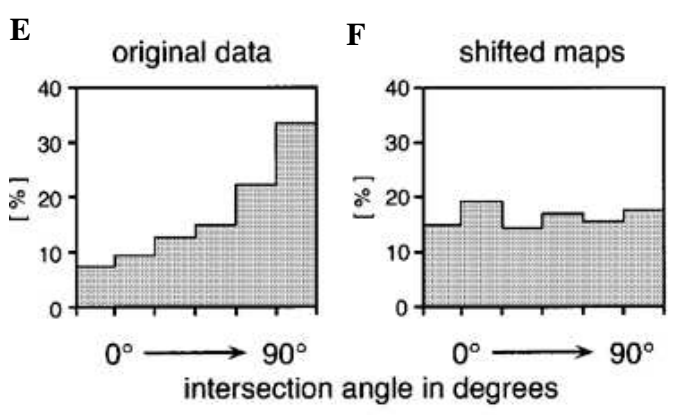

Figure 1.5: Segregated ODCs and OPM in strabismic cat V1 (A,B) Activity patterns for the left(A) and right eye (B) are complementary. Regions highly activated by left eye (dark regions in A, outlined in white) are only weakly activated by the right eye (light grey regions in B). (C) Orientation preference map obtained in the same area. (D) Superposition of the angle map and the outlined borders of adjacent ocular dominance columns (white contours in A). (E) Histograms of intersection angles between iso-orientation and ocular dominance columns in the same animal. Note that intersection angles between 75 and 90 are most abundant in the original data. (F) Data from shifted maps: iso-orientation contours of one animal superimposed with the ocular dominance borders of another animal. Note in addition that the histograms are always flat after shifting the maps (Data not shown here). Modified from Löwel et al. (1998). 
space dimensionalities, and visual stimulus distributions.

The phase transition to spontaneous pattern formation of visual maps was characterized by a stability analysis of the Kohonen model. The bifurcation diagrams were obtained numerically by varying the control parameter $\sigma$, which corresponds to the size of co-activated cortical domains. Large scale simulations were then performed in different dynamical regimes identified from the bifurcation diagrams.

In simulations the model cortex was initialized with an unselective state and was trained by random stimuli drawn from Gaussian distributions. We first considered the development of orientation preference interacting only with retinotopy. The dynamics of OPMs was found to rely on the size of the system. In small systems of one hypercolumn, a checkerboard pattern consisting of four pinwheels was always maintained. However, in the larger systems the initially pinwheel-rich patterns were typically unstable. The kinetics of pinwheel annihilation was quantified by the average numbers of pinwheels per hypercolumn, which decayed below 2 in various parameter regimes.

In simulations of high-dimensional feature space models, more feature dimensions were included to test whether pinwheel annihilation could be stopped as suggested by the dimension reduction framework. Intriguingly, only two active feature dimensions were represented close to the bifurcation threshold. The other feature dimensions were suppressed and became represented only beyond a secondary bifurcation point. Beyond this secondary bifurcation point, the generated patterns of different feature maps were either pinwheel-free stripes or a repetitive checkerboard pattern of pinwheel crystals.

To establish the robustness of this behavior we further compared simulations performed with different stimulus statistics. Similar results were found for spherical uniform distributions and for products of angular variables with circular uniform distributions. We conclude that the dynamics of OPMs is generally unstable in current developmental models of interacting columnar systems.

The first part of this thesis is organized as follows: In Chapter 22, we first describe the model of Kohonen's self-organizing feature mapping. Then we readdress the question of pattern formation by a linear stabilitv analvsis (Wolf et al., 2000) and derive the initial growth rate and the maximum unstable wavelength (related to the column spacing) of the emerging pattern. Based on this mathematical analysis, we designed a precisely controlled numerical method in Chapter 3, In Chapter 4, we study the dynamics of OPM interacting only with a retinotopic map. More feature dimensions are included in Chapter 5 to track the development of OPMs coupled to other feature maps. Finally, simulation results with stimuli of non-Gaussian distributions are described in Chapter 6. We discuss the main findings of our study in Chapter 7. 


\section{Chapter 2}

\section{Analysis of the Kohonen Model}

\subsection{Model Description}

The cortical surface is modeled as a two-dimensional layer which is divided into units of functional modules with common response properties. Each unit has an associated receptive field, which defines how it responds to different visual stimuli. Properties of receptive fields at the cortical location $\mathbf{x}$ are characterized by a feature vector $\mathbf{w}(\mathbf{x})$, whose components denote the preference for certain stimulus features.

In the following, we will use a two-component vector $\mathbf{p}=\left(p_{x}, p_{y}\right)$ to indicate the position of the receptive field centers in the visual space. We choose another two-component vector $\mathbf{z}=\left(z_{1}, z_{2}\right)$ to represent the orientation selectivity, with the angle of the vector $\phi(\mathbf{z})$ specifying the preferred orientation $\theta=\phi / 2$ and the length of the vector $|\mathbf{z}|$ indicating the corresponding tuning strength. The order parameter $\mathbf{z}(\mathbf{x})$ as a function of cortical position $\mathbf{x}$, describes the spatial pattern of orientation preferences. These properties are encoded by a four-dimensional feature vector

$$
\mathbf{w}(\mathbf{x})=\left(p_{x}, p_{y}, z_{1}, z_{2}\right)=(\mathbf{p}, \mathbf{z}),
$$

which can be simply extended for more features like ocular dominance and frequency selectivity, etc..

The feature properties of a stimulus are described by a vector $\mathbf{v}$ of the same dimensionality as $\mathbf{w}(\mathbf{x})$, with the corresponding components

$$
\mathbf{v}=\left(r_{x}, r_{y}, s_{1}, s_{2}\right)=(\mathbf{r}, \mathbf{s}) .
$$

After each presentation of the stimulus vector $\mathbf{v}$, the cortical activity pattern $e(\mathbf{x})$ as described below induces a modification of cortical receptive fields $\delta \mathbf{w}(\mathbf{x})$, which is driven towards the stimulus pattern

$$
\delta \mathbf{w}(\mathbf{x}) \propto[(\mathbf{v}-\mathbf{w}(\mathbf{x})) e(\mathbf{x})] .
$$


Eq.(2.3) can be derived from Hebbian dynamics of synaptic connections refined by correlated pre- and post-synaptic activity, and non-Hebbian terms which ensure that a measure of total svnaptic strength is conserved (Löwel \& Wolf.,1999: Wolf et al., 2000).

To enforce a continuous cortical mapping such that neighboring cortical columns have similar selectivities, co-activated cortical domains (CCDs) are assumed to dominate the cortical activity pattern $e(\mathbf{x})$. In response to an individual stimulus, the excitation of cortical neurons spreads locally within a CCD, which is simply taken as a stereotyped activity blob

$$
e(\mathbf{x})=\frac{1}{2 \pi} \exp \left(-\frac{\left|\mathbf{x}-\mathbf{x}^{*}\right|^{2}}{2 \sigma^{2}}\right),
$$

where $\mathbf{x}^{*}$ is the position of the most activated neuron and $\sigma$ measures the size of the CCD. For simplicity, we assume that afferent stimuli determine only the center location $\mathbf{x}^{*}$ of the CCD but not its shape and size. A competitive learning rule is used, choosing the winner position $\mathbf{x}^{*}$ with the feature vector $\mathbf{w}(\mathbf{x})$ closest to the current input $\mathbf{v}$ :

$$
\left|\mathbf{v}-\mathbf{w}\left(\mathbf{x}^{*}\right)\right| \leq|\mathbf{v}-\mathbf{w}(\mathbf{x})| \quad(\text { for all } \mathbf{x})
$$

Note that Eq.(2.4) is adopted from Kohonen's algorithm; however, other learning rules could easily be incorporated to update the receptive fields of CCDs, and the concrete forms of excitation pattern are not expected to affect the key feature of the activity dependent mechanism.

To study the dynamics of OPMs, we rewrite Eq.(2.3) decomposing orientation preference $\mathbf{z}(\mathbf{x})$ from the feature vector $\mathbf{w}(\mathbf{x})$ and $\mathbf{s}$ from the stimulus vector $\mathbf{v}$ :

$$
\delta \mathbf{z}(\mathbf{x}) \propto[(\mathbf{s}-\mathbf{z}(\mathbf{x})) e(\mathbf{x})]
$$

If modifications induced by individual stimuli are small enough in the sense that presenting a single input stimulus should not globally disturb the present spatial pattern, $\mathbf{z}(\mathbf{x})$ changes slowly through the cumulative effect of a large number of activity events. Its temporal evolution dynamics is then described by a deterministic equation:

$$
\frac{\partial}{\partial t} \mathbf{z}(\mathbf{x})=\langle(\mathbf{s}-\mathbf{z}(\mathbf{x})) e(\mathbf{x})\rangle
$$

where $t$ denotes time and \langle\rangle represents the average over an ensemble of afferent activity patterns. Cortical activity $e(\mathbf{x})$ takes the form of Eq. (2.4) with the winner position

$$
\mathbf{x}^{*}=\arg \min \left(|\mathbf{s}-\mathbf{z}(\mathbf{x})|^{2}+|\mathbf{r}-\mathbf{p}(\mathbf{x})|^{2}\right)
$$

such that the closest distance between the stimulus vector and the receptive field feature vector is found at cortical position $x^{*}$. 
The dynamics of $\mathbf{z}(\mathbf{x})$ defined by Eqs.(2.4), (2.7) and (2.8) has a homogenous stationary solution $\mathbf{z}_{0}(\mathbf{x})$. If we assume the stimulus ensemble has no bias to any orientation, the homogeneous state is unselective with respect to different orientations:

$$
\mathbf{z}_{0}(\mathbf{x})=\langle\mathbf{s}\rangle=(0,0)
$$

\subsection{Linear Stability Analysis}

To understand the condition for the generation of an orientation preference map and to determine the initial rate of pattern growth, we explored whether the stationary state $\left(\mathbf{z}_{0}(\mathbf{x})=\mathbf{0}\right)$ was stable or not in the learning process defined above. When the homogeneous solution loses stability, orientation selectivity will emerge spontaneously. Following Wolf's work on the dynamics of cortical pattern formation (Wolf \& Geisel, 1998; Wolf et al., 2000), we used linear stability analysis to investigate the dynamics of OPM starting from an unselective homogenous state.

From Section 2.1 the dynamics of OPM $\mathbf{z}(\mathbf{x})$ can be summarized as

$$
\left\{\begin{array}{l}
\frac{\partial}{\partial t} \mathbf{z}(\mathbf{x})=\langle(\mathbf{s}-\mathbf{z}(\mathbf{x})) e(\mathbf{x})\rangle \\
e(\mathbf{x})=\frac{1}{2 \pi} \exp \left(-\frac{\left|\mathbf{x}-\mathbf{x}^{*}\right|^{2}}{2 \sigma^{2}}\right) \\
\mathbf{x}^{*}=\arg \min \left(|\mathbf{s}-\mathbf{z}(\mathbf{x})|^{2}+|\mathbf{r}-\mathbf{x}|^{2}\right)
\end{array}\right.
$$

where $\sigma$ measures the size of a co-activated cortical domain (CCD) and \langle\rangle represents the temporal average over an ensemble of afferent activity patterns.

Without loss of generality, the retinotopic map $\mathbf{p}(\mathbf{x})$ is rescaled to share a common coordinate system with cortical position $\mathbf{x}$. At the first step, we assume that initially there exists an isotopic topological organization which would not be disrupted by the emergence of the orientation map

$$
\mathrm{p}(\mathrm{x})=\mathrm{x} .
$$

To determine the local stability, we linearize the dynamics of $\mathbf{z}(\mathbf{x})$ around $\mathbf{z}_{0}(\mathbf{x})$. Because the resulting linear equation must be translation invariant in the cortical layer, its eigenfunctions are plane waves. It is therefore sufficient to study the stability of the model in one spatial dimension

$$
\begin{aligned}
\frac{\partial}{\partial t} z(x) & =\frac{1}{2 \pi}\left\langle(s-z(x)) \exp \left(-\frac{\left|x-x^{*}\right|^{2}}{2 \sigma^{2}}\right)\right\rangle \\
& =\frac{1}{2 \pi} \int \mathrm{d} s \mathrm{~d} r P(s)(s-z(x)) \exp \left(-\frac{\left|x-x^{*}\right|^{2}}{2 \sigma^{2}}\right),
\end{aligned}
$$


where $x^{*}=x^{*}(r, s, z(\cdot))$ is the center of the activated domain.

To eliminate the implicit dependence of the activity center $x^{*}$ on the stimulus parameters $r, s$ and $z(x)$,we perform a coordinate transform, and represent $r$ and $s$ with new variables $u$ and $v$.

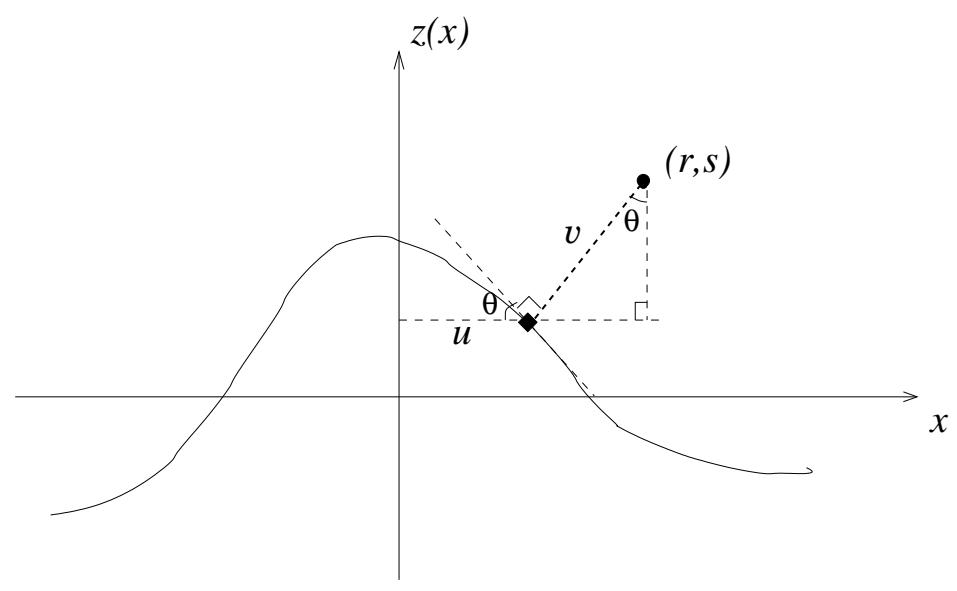

Figure 2.1: Coordinate transform The stimulus parameters $r$ and $s$ are represented by new variables $u$ and $v$, where $v=\min \left(\sqrt{|\mathbf{s}-\mathbf{z}(\mathbf{x})|^{2}+|\mathbf{r}-\mathbf{x}|^{2}}\right)$ re-evaluates the smallest Euclidean distance between the stimulus vector and all feature vectors, while $u=x^{*}$ is the corresponding winner position

From Figure 2.1 we rewrite $r, s$ as

$$
\begin{aligned}
& r(u, v)=u+v \sin \theta=u-\frac{v z_{x}(u)}{\sqrt{z_{x}^{2}(u)+1}}, \\
& s(u, v)=z(u)+p \cos \theta=z(u)+\frac{v}{\sqrt{z_{x}^{2}(u)+1}} .
\end{aligned}
$$

To know the corresponding Jacobian

$$
J=\operatorname{det}\left(\begin{array}{ll}
r_{u} & r_{v} \\
s_{u} & s_{v}
\end{array}\right),
$$

we first compute the partial derivatives:

$$
\begin{array}{ll}
r_{u}=1-\frac{v z^{\prime \prime}}{\Delta}+\frac{v z^{\prime 2} z^{\prime \prime}}{\Delta^{3}}, & r_{v}=-\frac{z^{\prime}}{\Delta}, \\
s_{u}=z^{\prime}-\frac{v z^{\prime \prime} z^{\prime}}{\Delta^{3}}, & s_{v}=\frac{1}{\Delta},
\end{array}
$$

where $z^{\prime}=z_{x}(u), z^{\prime \prime}=z_{x x}(u), \Delta=\sqrt{z_{x}(u)^{2}+1}$. 
By substituting the partial derivatives, performing a Taylor expansion in $z(x)$ and neglecting the higher order terms of $z^{\prime}$ and $z^{\prime \prime}$, we obtain

$$
\begin{aligned}
J=r_{y} s_{p}-r_{p} s_{y} & =\sqrt{z^{\prime 2}+1}-\frac{p z^{\prime \prime}}{1+z^{\prime 2}} \\
& \simeq 1+z^{\prime}-p z^{\prime \prime}=1+z_{x}(u)-p z_{x x}(u) .
\end{aligned}
$$

The dynamics close to the homogeneous stationary state $z_{0}(x)$ can therefore be written as

$$
\begin{aligned}
\frac{\partial}{\partial t} z(x)=\frac{1}{2 \pi} \int & \mathrm{d} u \mathrm{~d} v \hat{P}(u, v) J\left(v, z_{x}(u), z_{x x}(u)\right)(s(u, v)-z(x)) \\
& \times \exp \left(-\frac{|x-u|^{2}}{2 \sigma^{2}}\right) .
\end{aligned}
$$

Since we are interested in the dynamics near the fixed point, i.e. $z_{x}(u) \ll 1$, we linearize the coordinate transform

$$
\begin{aligned}
& r(u, v)=u-\frac{v z_{x}(u)}{\sqrt{z_{x}^{2}(u)+1}} \simeq u-v z_{x}(u), \\
& s(u, v)=z(u)+\frac{u}{\sqrt{z_{x}^{2}(u)+1}} \simeq z(u)+v .
\end{aligned}
$$

The probability density function of the stimulus set can be represented with new variables:

$$
\hat{P}(u, v):=P\left(u-v z_{x}(u), z(u)+v\right) .
$$

We then perform a Taylor expansion and neglect the higher order terms of $z(u), z_{x}(v)$

$$
\hat{P}(u, v) \simeq P(u, v)-P_{r}(u, v) v z_{x}(u)+P_{s}(u, v) z(u) .
$$

Because of translation symmetry, $P(s, r)$ is independent of the position $P(s, r)=$ $P(s)$, hence the second term should vanish:

$$
\hat{P}(u, v) \simeq P(u, v)+P_{s}(u, v) z(v) .
$$

The right hand side of the integro-differential Eq.(2.15) can then be linearized by linearizing the integrand

$$
\begin{aligned}
& \hat{P}(u, v) J\left(v, z_{x}(u), z_{x x}(u)\right)(s(u, v)-z(x)) \\
= & \left(P(u, v)+P_{s}(u, v) z(u)\right)\left(1+z_{x}(u)-v z_{x x}(u)\right)(z(u)+v-z(x)), \\
= & P(u, v)(z(u)-z(x))-P(u, v) v^{2} z_{x x}(u)+P_{s}(u, v) v z .
\end{aligned}
$$

After performing the $v$ integration in Eq.(2.15) we obtain

$$
\begin{aligned}
\frac{\partial}{\partial t} z(x) & =\frac{1}{2 \pi} \int \mathrm{d} u \exp \left(-\frac{|x-u|^{2}}{2 \sigma^{2}}\right)\left(-z(x)-\left\langle v^{2}\right\rangle z_{x x}(u)\right) \\
& =-\frac{\sigma}{\sqrt{2 \pi}} z(x)-\frac{\left\langle v^{2}\right\rangle}{2 \pi} \int \mathrm{d} u \exp \left(-\frac{|x-u|^{2}}{2 \sigma^{2}}\right) z_{x x}(u)
\end{aligned}
$$


The growth rates of $z(x)$ are given by the Fourier transform of Eq.(2.16) as the eigenvalues of the rhs operator:

$$
\lambda(k)=\frac{\sigma}{\sqrt{2 \pi}}\left(-1+\sigma_{s}^{2} k^{2} \exp \left(-\frac{k^{2} \sigma^{2}}{2}\right)\right) .
$$

The spectrum (Figure 2.2) has a single maximum at wavenumber

$$
k_{\max }=\frac{\sqrt{2}}{\sigma}
$$

and is positive when

$$
\sigma<\sigma^{*}=\sqrt{\frac{2\left\langle v^{2}\right\rangle}{e}} .
$$

The corresponding maximum unstable wavelength

$$
\Lambda_{\max }=\frac{2 \pi}{k_{\max }}=\sqrt{2} \pi \sigma
$$

with the fastest growth rate will initially dominate the emerging pattern, and is called the critical wavelength in the following.

Next we consider the development of $\mathbf{z}(\mathbf{x})$ coupled to changes of the retinotopic map, the resulting linearized dynamics may in principle contain an additional first order term of $\delta \mathbf{z}(\mathbf{x})$ :

$$
\frac{\partial}{\partial t} \delta \mathbf{z}(\mathbf{x})=\widehat{L_{\mathbf{z}}} \delta \mathbf{z}(\mathbf{x})+\widehat{L_{\mathbf{p}}} \delta \mathbf{p}(\mathbf{x})
$$

Imagine that we inverse the coordinate of the retinotopic map $\delta \mathbf{p}(\mathbf{x}) \rightarrow-\delta \mathbf{p}(\mathbf{x})$. This leaves invariant the uniform distribution of afferent stimuli on retinal position. Based on the orthogonal assumption between different feature directions, the dynamics of $\delta \mathbf{z}(\mathbf{x})$ should remain unchanged:

$$
\frac{\partial}{\partial t} \delta \mathbf{z}(\mathbf{x})=\widehat{L_{\mathbf{z}}} \delta \mathbf{z}(\mathbf{x})-\widehat{L_{\mathbf{p}}} \delta \mathbf{p}(\mathbf{x})
$$

From Eq.(2.21), (2.22), we thus know that

$$
\widehat{L_{\mathbf{p}}}=0
$$

Thus, in the linear regime, changes of the retinotopic map do not affect the dynamics of the orientation map. The existence of a critical size $\sigma^{*}$ of coactivated cortical domain calculated above can be applied to more general cases with coupled retinotopic dynamics. 


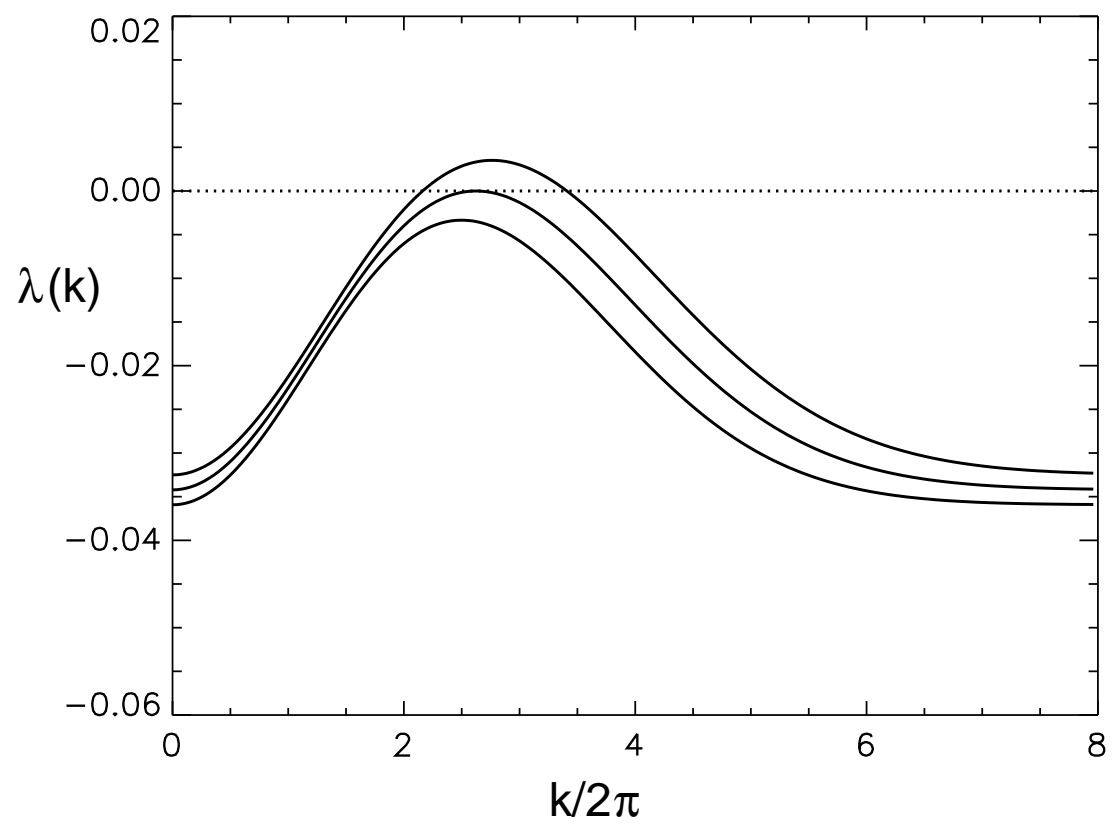

Figure 2.2: Representative spectra of pattern growth rates $\lambda(k)$ for $\left\langle p^{2}\right\rangle=$ 0.01 and $\sigma=1.05 \sigma^{*}, \sigma^{*}, 0.95 \sigma^{*}$ from bottom to top. For $\sigma$ smaller than $\sigma^{*}$, the largest growth rate is positive and hence leads to instability of the homogeneous state and the spontaneous emergence of a finite wavelength pattern. 


\section{Chapter 3}

\section{Numerical Methods}

\subsection{Simulation Procedure}

The two-dimensional model cortex as previously described, was represented on an $N \times N$ square grid indexed by $(i, j)$ with $0 \leq i, j \leq N-1$. To achieve a good approximation of the spatially continuous pattern, the density of grid points was chosen to include at least 10 pixels per column spacing. In the primary visual cortex of a cat, the typical spacing of iso-orientation domains is roughly $1 \mathrm{~mm}$; the corresponding spatial resolution is thus equivalent to $100 \mu \mathrm{m}$. Periodic boundary conditions were applied to avoid edge effects and to conserve the translation invariance of the spatial pattern.

The receptive field of each grid point consisting of two retinal dimensions plus $n$ additional feature dimensions $(2 \mathrm{D}+\mathrm{nD})$, is given by $\mathbf{w}_{i, j}=\left(x, y, z_{1}, z_{2}, \ldots, z_{n}\right)$. Initially, a roughly ordered retinotopic map was assumed to be present, with $x_{i, j}=i L /(N-1)+\xi_{x}$ and $y_{i, j}=j L /(N-1)+\xi_{y}$, where $\xi_{x}$ and $\xi_{y}$ are Gaussian random numbers with a mean of zero and a standard deviation of 0.005 . $L$ measures the linear extent of the retinal region mapped onto the cortex, which was normalized to 1 in our simulation. All the other feature values $z_{1}, z_{2}, \ldots$, and $z_{n}$ were initialized as zero.

Stimuli defined by a vector $\mathbf{v}_{i, j}=\left(x_{s}, y_{s}, s_{1}, s_{2}, \ldots, s_{n}\right)$ were chosen at random according to a predefined density in the feature space: retinal position values $x_{s}$ and $y_{s}$ were distributed uniformly in the interval $[0, L]$. The values of $s_{1}, s_{2}, \ldots$, and $s_{n}$ were either independently drawn from the same Gaussian distribution with the mean of zero and the standard deviation of $\sigma_{s}$ or from a non-Gaussian stimulus distributions as discussed in more details in Chapter 6. For maps of spherical feature space, the stimulus values were uniformly distributed on the $n$-dimensional sphere of radius $\sqrt{2} \sigma_{s}$. For maps of multiple circular variables, the values of $s_{1}, s_{2}, \ldots$, and $s_{n}$ were grouped into $n / 2$ pairs and each pair was uniformly distributed on a circle of radius $\sqrt{2} \sigma_{s}$.

At each step one random stimulus was presented, whereas the cortical recep- 
tive fields changed by an amount

$$
\delta \mathbf{w}(\mathbf{x})=\varepsilon(\mathbf{v}-\mathbf{w}(\mathbf{x})) e(\mathbf{x})
$$

where $\varepsilon$ is the 'learning' rate factor that defines the size of an integration time step $\delta t=\varepsilon$. To choose $\varepsilon$ appropriately, this time step must be compared to the intrinsic time scale $\tau$ of the dynamics that is the inverse of the fastest growth rate $\lambda_{\max }$ with which the emerging pattern grows exponentially

$$
\tau=\frac{1}{\lambda_{\max }}
$$

The maximum eigenvalue $\lambda_{\max }$ was obtained from the linear stability analysis around the initial homogenous state (see Section 2.2). The total amount of stimuli presented for a period of $\tau$ is then given by $\tau / \varepsilon$.

Simulations were performed repeatedly by seeding the random number generator to obtain different stimulus sets. Time was measured in the units of $\tau$. It is important to note that, in order to answer the question whether the pinwheels generated in the initial symmetry breaking phase are stable or not, one must integrate the dynamics of Eq.(3.1) over a time period much longer than the intrinsic time scale $\tau$.

\subsection{Choosing the Learning Rate}

For the result of such a stochastic updating to approximate the deterministic dynamics in Eqs 2.10, we chose the learning rate factor $\varepsilon$ to fulfill two statistical requirements within a time interval of $\tau$. First, every patch on the cortical layer has to be activated by a sufficient number of stimuli. Second, a sufficient number of stimuli should be drawn from each voxel of the feature space to estimate the input vector statistics.

Since input vectors have equal variances $\sigma_{s}^{2}$ in all the $n$-dimensions of the feature space, we partitioned the relevant volume of the feature space $V_{\text {total }}=$ $\left(2 \sigma_{s}\right)^{n}$ into voxels of size $V_{\text {voxel }}=\left(\sigma_{s} \epsilon_{s}\right)^{n}$, where $\epsilon_{s}$ is the spatial resolution in the feature space. If we further assumed the temporal average of the afferent activity patterns had a uniform distribution across the cortical layer, the number of stimuli from a typical voxel in the feature domain to activate each cortical hypercolumn is

$$
N_{s}=\frac{\tau}{\varepsilon \Gamma^{2}}\left(\frac{\epsilon_{s}}{2}\right)^{n}
$$

where the aspect ratio of the system

$$
\Gamma=\frac{L}{\Lambda_{\max }}
$$

measures the system size of the cortical domain in the scale of its characteristic column spacing $\Lambda_{\max }$. 
To resolve the overall structure of the input vector distribution in the feature space, we required $\epsilon_{s}=0.2, N_{s}=100$. According to Eq. (3.5), the learning rate $\varepsilon$ has to be reduced when increasing the aspect ratio $\Gamma$ of the cortical domain or the dimensionality $n$ of the feature space according to

$$
\varepsilon=\frac{\tau}{N_{s} \Gamma^{2}}\left(\frac{\epsilon_{s}}{2}\right)^{n}
$$

in order to maintain the same noise level.

\subsection{Principal Component Analysis}

Principal component analysis (PCA) was used to characterize the representation of high dimensional feature spaces. Based on linear transformation, PCA chooses a coordinate system for a data set such that the greatest variance by any projection of the data set comes to lie on the first axis (so called the first principal component), the second greatest variance on the second axis, and so on. By such a statistical approach, we identified dimensions in the simulated receptive field features that contribute most to its variance.

We first computed the covariance of different feature components averaged over the entire cortex. The covariance matrix $\mathbf{A}$ is a $n \times n$ matrix given by $A_{i j}=\left\langle z_{i} z_{j}\right\rangle$. The method of singular value decomposition was applied to evaluate the eigenvalues of $\mathbf{A}$ :

$$
\mathbf{A}=\mathbf{B M B}^{T}
$$

where $\mathbf{M}=\operatorname{diag}\left(m_{1}, m_{2}, \ldots, m_{n}\right)$ yields the variances of the data under the coordinate transform $\mathbf{z}^{\prime}=\mathbf{B}^{T} \mathbf{z}$.

\subsection{Rotation of the Feature Coordinate System}

To avoid the computer round-off error accumulating on the main axes which might cause breaking of the symmetry, orthogonal transformations were applied during the simulation shown in Figure 5.1D to choose different coordinate systems for computation. The feature vector was multiplied by an arbitrary orthogonal matrix and its inverse before and after every hundred step of numerical integration, respectively, such that the lengths of feature vectors and the angles between vectors were preserved.

\subsection{Map Analysis}

\section{Color Code}

We used a standard linear scaled color code of 16 colors to visualize orientation maps. Due to the symmetry assumption, all different orientations are expected 
to be equally represented on the model cortex. The map dynamics remains unaffected by shift of orientations. Hence in principle the color code of individual maps can be shifted by an arbitrary angle yielding an equivalent solution.

\section{Pinwheel Finding}

The preferred orientation was deduced from the vector field $\mathbf{z}(\mathbf{x})$ according to $\theta=\frac{1}{2} \arctan \left(z_{1} / z_{2}\right)$. Pinwheels were identified as the crossing points between the contour lines defined by $z_{1}=0$ and $z_{2}=0$. The number of pinwheels $n_{p w}$ and the location of pinwheels were calculated using code developed bv Wolf (Löwel et al., 1998).

\section{Topological Charge}

The topological charge $q$ is a topologically conserved quantity for continuously changing maps. In the case of OPMs, it is defined as the change of prefered orientation integrated along a closed circle $C$ :

$$
q=\frac{1}{2 \pi} \oint_{C} \nabla \theta(\mathbf{x}) \mathrm{d} \mathbf{s} .
$$

Since $\theta(\mathbf{x})$ is a continuous function of $\mathbf{x}$ up to the isolated singular points of pinwheels, the topological charge of pinwheel-free regions is 0 . Around a pinwheel center, the change of prefered orientations sums up to $\pi$, which yields a topological charge of $\pm 1 / 2$. Thus there are only two types of pinwheels in the model, around which either the orientation preference increases clockwise $(q=-1 / 2)$ or counterclockwise $(q=1 / 2)$. Higher topological charges of $q= \pm 1, \pm 3 / 2, \ldots$ are unstable under small perturbations (called structurally unstable in mathematical terms) and have never been observed in experiments.

If the dynamics of OPMs is continuous over time, the total topological charge $Q_{A}$ of a given bounded area A is conserved. Since the map contains pinwheels with $q= \pm 1 / 2$, only two possible mechanisms can alter the number of pinwheels in a given area A: either generation of two pinwheels showing opposite charges, or annihilation of two pinwheels with opposite charges.

\section{Nearest Neighbor Distance}

In some of the maps, we calculated the distance $h$ of each pinwheel to its nearest neighbours. We first computed the distance between each other from the location of pinwheels. Next, for each pinwheel, we found the closest distance to another pinwheel, either with the same sign of charge $\left(h_{+}\right)$or with opposite sign of charge $\left(h_{-}\right)$. The distances $h$ taking the smaller of the two distances, measures the closest distances among all neighbors, regardless of topological charges. Distributions of $h_{+}, h_{-}$, and $h$ indicate the regularity and periodicity of the maps. 


\section{Column Spacing and Pinwheel Density Estimation}

The typical column spacing $\Lambda$ can be obtained by calculating the average wave number based on the Fourier transform:

$$
\begin{gathered}
\Lambda=2 \pi / \bar{k}, \\
\bar{k}=\int \mathrm{d} k k P(k) / \int \mathrm{d} k P(k),
\end{gathered}
$$

where $P(k)=|\widetilde{\mathbf{z}}(k)|^{2}$ is the power spectral density of $\mathbf{z}(\mathbf{x})$.

Pinwheel density was measured as the numbers of pinwheels per hypercolumn. Hence, pinwheel density is weighted by the size of maps in units of $\Lambda^{2}$. The scaled pinwheel density is then given by

$$
\hat{\rho}=n_{p w} \Lambda^{2} / L^{2}
$$




\section{Chapter 4}

\section{Dynamics of OPMs in 2D Models}

In this chapter we study the development of OPMs based on the numerical methods described in the previous chapter. The linear stability analysis in Chapter 2 predicts that maps develop from an initial unselective state only if the size of co-activated cortical domains is below a critical value $\sigma^{*}=\sigma_{s} \sqrt{2 / e}$.

In the following we first study this critical dependance of pattern formation in simulations varying the value of $\sigma$. The bifurcation diagram is obtained by plotting the average orientation tuning strength versus $\sigma$. Further simulations are performed to characterize different parameter regimes identified in the bifurcation diagram. The temporal dynamics of OPMs is found dependent on the size of stimulus set. Moreover, different behaviors are observed in different dynamical regimes and in systems of different sizes. At the end we quantify the kinetics of pinwheel annihilation in different systems.

\subsection{Spontaneous Map Formation}

In order to reveal the critical point for map formation, we fixed the stimulus variance $\sigma_{s}=0.133$ and performed simulations with different values of $\sigma$. From the theoretical prediction, the pattern should spontaneously emerge at $\sigma^{*}=$ $\sigma_{s} \sqrt{2 / e}=0.114$.

Figure 4.1 shows two simulation examples. Given a larger value of $\sigma=0.2$ (Figure 4.1A, upper panel) the OPM has no repetitive structure and the pattern changes abruptly and globally after presentation of each stimulus. In contrast, the map generated with a value of $\sigma=0.1$ (Figure 4.1, lower panel) seems more stable and the pattern resembles features of an experimentally observed map.

For a quantitative comparison, we plotted the temporal evolution of the average tuning strength of these two maps in Figure 4.1B, which shows a significant degree of orientation selectivity with $\sigma=0.1$ but only small fluctuation with $\sigma=0.2$.

We also tracked the changes of orientation selectivity at an arbitrarily chosen 
position on the map (Figure 4.17A, open circles), plotted in Figure 4.17C. In the simulation with $\sigma=0.2$, the cortical unit had no consistently preferred orientation but rather jumped between all different orientations. In contrast to that, a specific orientation (around 130 degree in this example) was selected in the simulation with $\sigma=0.1$.
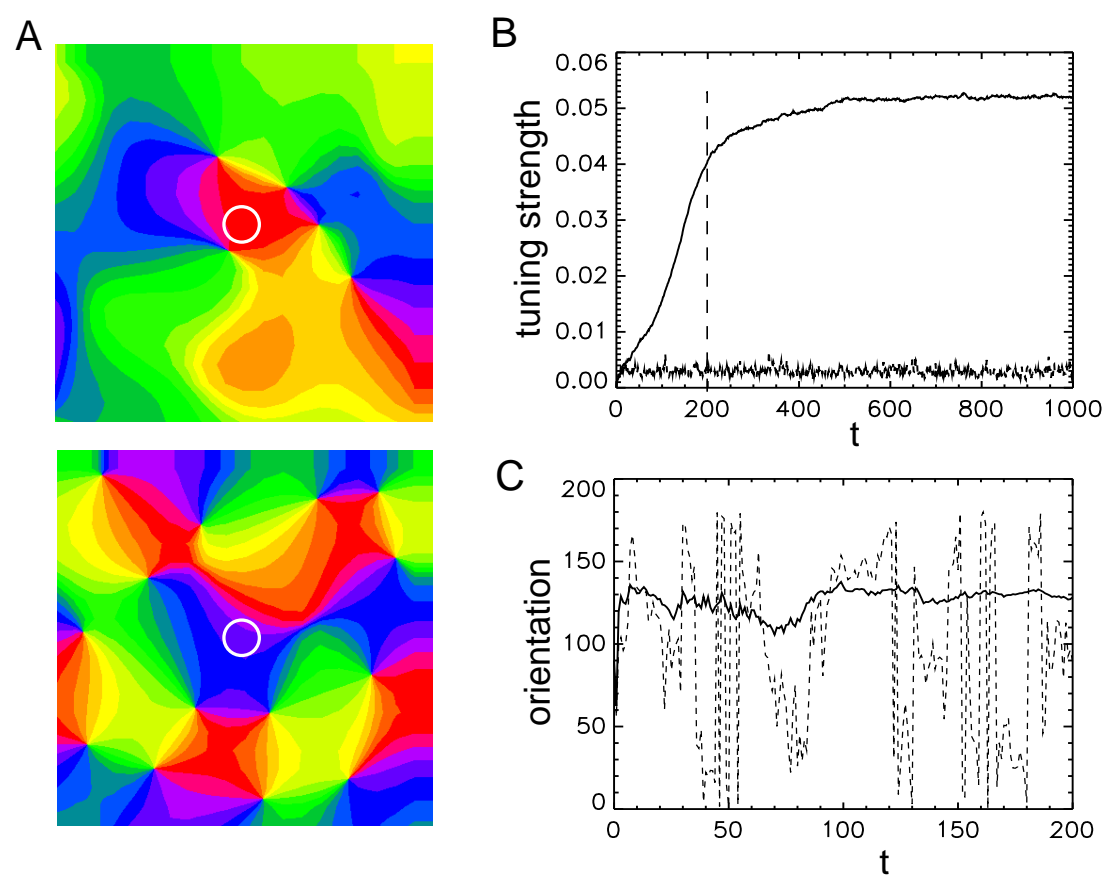

Figure 4.1: Emergence of orientation preference. (A) Examples of angle maps generated by simulations with $\sigma_{s}=0.133, \sigma=0.2$ (upper panel), and $\sigma=0.1$ (lower panel), at integration time $t=200$. (B) Orientation tuning strengths averaged over the entire grid are plotted against time. The dotted curve is from the simulation with $\sigma=0.2$ while the solid curve is from the simulation with $\sigma=0.1$. The vertical dashed line indicates the time when maps in (A) are recorded. (C) At an arbitrarily selected position indicated with open circles on the angle maps, the changes of preferred orientations are represented by the dash line for $\sigma=0.2$ and the solid line for $\sigma=0.1$. Time is measured in units of simulation steps multiplied by the learning rate $t=\varepsilon \cdot i$.

In order to construct a bifurcation diagram, we computed the changes of the average tuning strength in simulations with different values of $\sigma$. Figure $4.2 \mathrm{~A}$ shows that near the threshold $\sigma^{*}=0.114$, the strength of orientation selectivity abruptly decreases with increasing values of $\sigma$. We plot the plateau values of the average tuning strength versus $\sigma$ in Figure $4.2 \mathrm{~B}$. The tuning strength reduces to about zero at $\sigma=0.115$, which precisely agrees with the theoretical prediction of $\sigma^{*}=0.114$ 
To reveal the qualitatively different behaviors above and below the critical point, we performed further simulations with smaller learning steps $\varepsilon$ to reduce the noise level in the simulations. The amplitude of orientation tuning for $\sigma<$ 0.115 remained unaffected; with larger values of $\sigma$ it decreased proportional to $\varepsilon$, which is characteristic of fluctuations around a stable homogenous state.
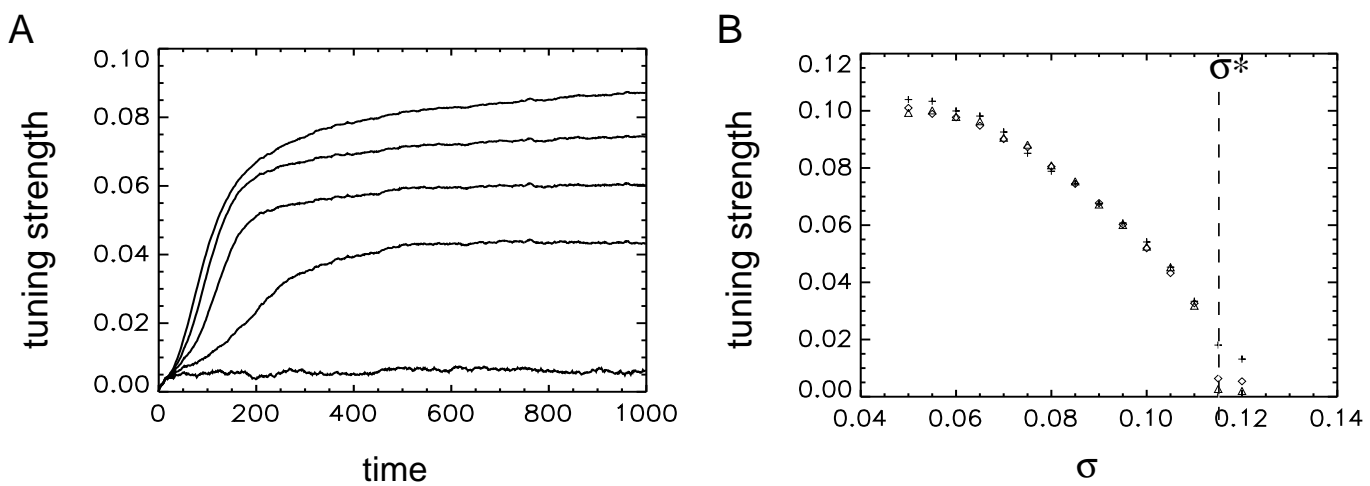

Figure 4.2: The tuning strength of OPMs varies with size of CCDs (A) Orientation tuning strengths that averaged over the entire grid are plotted against the simulation time. From top to bottom: $\sigma=0.075,0.085,0.095,0.105,0.115$; $\sigma_{s}=0.133$. (B) The plateau values of the average tuning strength are plotted against the values of $\sigma$ between 0.05 and 0.12 . The crosses, open diamonds and triangles indicate simulations with different learning rates, $\varepsilon=0.01,0.001,0.0001$, respectively. Time is measured in units of simulation steps multiplied by the learning rate $t=\varepsilon \cdot i$.

Most of our subsequent simulations were performed with $\sigma=0.9 \sigma^{*}$ or $\sigma=$ $0.667 \sigma^{*}$. These two choices represented systems close to, or far from the symmetry breaking threshold, respectively.

\subsection{Maps 'Frozen' by a Small Set of Stimuli}

In cortical development the initially established circuitry is believed to require a progressive refinement that goes on for a couple of months to reach full maturity. Visual input plavs an instructive role during this period (for reviews see Sur \& Leamey (2001) and White \& Fitzpatrick (2007)). Motivated by this observation, we studied map dynamics on a time scale much longer than the intrinsic time scale for map formation.

For reasons of computational efficiency, a small set of fixed stimuli has often been used to train the network bv competitive Hebbian rules (Durbin \& Mitchison, 1990; Goodhill \& Cimponeriu, 2000). In these studies, a fixed set of stimuli 
were drawn only once and used repeatedly for the entire simulation. Such procedure might not adequately represent the biological activity-dependent process. Indeed in our simulation, the temporal evolution of map dynamics was found to be heavily influenced by the size of stimulus set.

Figure 4.3A shows a simulation initialized with a pinwheel-rich pattern. When trained with a small stimulus set, the map dynamics stopped evolution after $t \approx 100 \tau$ such that several pinwheels were preserved in the system. However, this pattern was unstable when using a larger stimulus set.

We started from the same initial condition as in Figure 4.3. $\mathrm{A}$ but instead presented the network with randomly generated stimuli (Figure 4.3B). Pinwheels vanished quite fast with only orientation stripes left.

Figure 4.3 $\mathrm{C}$ compares the kinetics of both simulations. The scaled pinwheel density dropped to zero with random stimuli but remained above 3 with a fixed set of stimuli. We used the analog of a physical term 'quenched disorder' here to describe such a phenomenon that maps were 'frozen' by presenting the same set of stimuli repeatedly.

We further analyzed the effect of quenched disorder by varying the size of the stimulus set. As shown in Figure 4.2, initialized with the same pinwheelrich pattern, the orientation maps underwent a learning process in which the characteristic structure of pinwheels was gradually lost. The process of pinwheel annihilation could be stopped if no new stimulus was recruited.

We conclude that presenting a small set of stimuli is insufficient to represent the entire feature space of random inputs and qualitatively changes the nature of the emerging map. It causes freezing of the map, stopping its temporal dynamics. In such simulations, the density of pinwheels in the frozen pattern depends strongly on the size of the stimulus set. Only few pinwheels are preserved when presenting a stimulus set resembling the complexity and richness of visual environment.

\subsection{Pinwheel Dynamics Depends on System Size}

The size of the system $\Gamma$, called aspect ratio, is measured by the number of repetitive units in the initial established pattern:

$$
\Gamma=\frac{L}{\Lambda_{\max }}=\frac{L}{\sqrt{2} \pi \sigma}
$$

In this section we simulated systems of different size by changing the value of $\sigma$ which determines the most unstable wavelength $\Lambda=\sqrt{2} \pi \sigma$ (see Section 2.2 for detailed calculation). The parameters of $\sigma$ and $\sigma_{s}$ were chosen to conserve their ratio fixing the parameter regime in the bifurcation diagram (Figure 4.2 B). Spatial patterns at the initial stage of map formation were compared with those at later stages in systems of various sizes. 

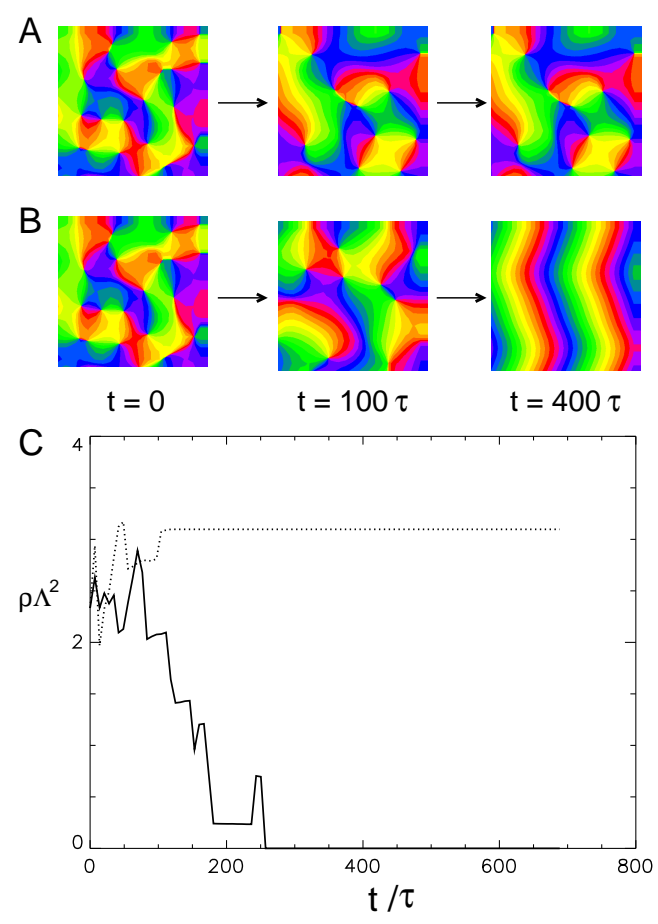

Figure 4.3: Maps 'frozen' by a small set of stimuli (A) The development of an orientation map $\left(\sigma=0.667 \sigma^{*}\right)$ initialized with a pinwheel-rich pattern. A fixed set of 1000 stimuli was presented repeatedly to train the system. (B) The initial condition was the same as in (A). Trained with non-repeating random stimuli, the pattern became unstable and stripes took over pinwheels. (C)The changes of scaled pinwheel density; the dashed line shows the process with a fixed stimulus set, while the solid line shows the process with random stimuli. 


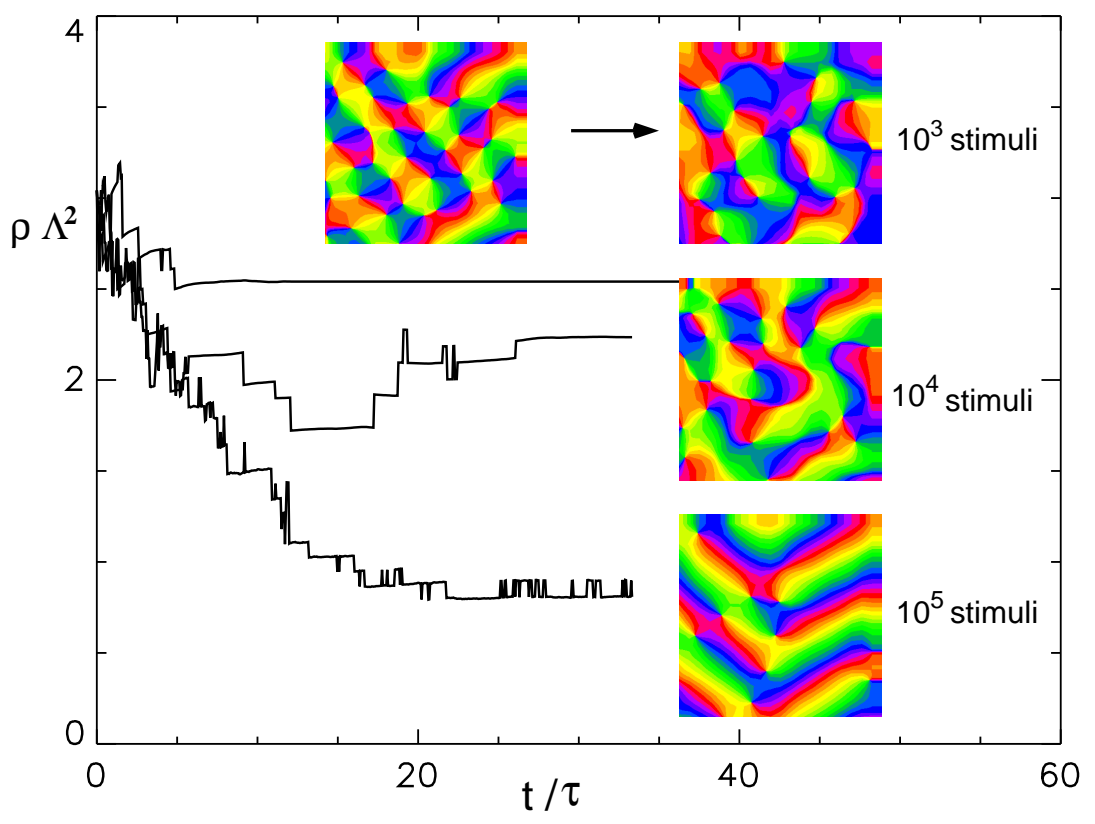

Figure 4.4: Dynamics vary with the size of the presented stimulus set. Kinetics of pinwheel annihilation $\left(\sigma=0.9 \sigma^{*}\right)$ for different sizes of stimulus set, starting from the same initial condition. From top to bottom, the system was trained with $10^{3}, 10^{4}$, and $10^{5}$ stimuli; the scaled pinwheel density dropped below 1.0 when more random stimuli were presented. Insets show the initial OPM (left column) and the map configurations at the end of each simulation (right column). The map appears more stripelike for a larger number of stimuli. 


\subsubsection{Dynamics in Small Systems}

We first investigated the dynamics of systems with a size of one single column spacing $\left(L=1 \Lambda_{\max }\right)$, i.e., only one hypercolumn represented in the model cortex.

The OPMs in these simulations resembled simple and regular checkerboardtype patterns, as shown in Figure 4.5A. Maps generated in different simulations showed quantitatively similar patterns, which could be superimposed by translation, rotation, and orientation shift of different maps.

In simulations such patterns converged quite fast to a steady state, with hardly any change on the spatial layout after $t>10 \tau$. Figure $4.5 \mathrm{~B}$ plots the growth of the average amplitude of the feature vectors. With $\sigma=0.667 \sigma^{*}$, the system was far away from the bifurcation point, resulting in a pattern with larger amplitude (Figure 4.5B B upper trace). The system close to the critical point $\left(\sigma=0.9 \sigma^{*}\right)$ showed a smaller magnitude of orientation tuning averaged over the map (Figure $4.5 \mathrm{~B}$, lower trace) as expected from the bifurcation diagram (Figure 4.2).

In both parameter regimes, the pattern of four-pinwheel configuration was very stable in every simulation, with the scaled pinwheel density $\rho \Lambda^{2}$ at a constant value of 4 (Figure 4.5C).
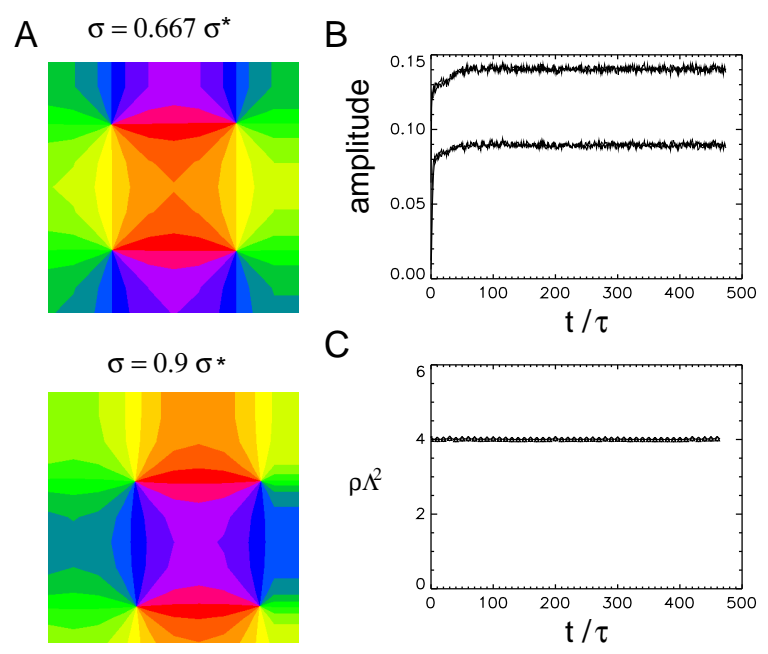

Figure 4.5: Dynamics in small systems $\left(L=1 \Lambda_{\max }, \mathbf{2}+\mathbf{2 D}\right.$ feature space) (A) Typical examples of OPMs obtained with different values of $\sigma$. (B) The amplitude of each feature component averaged over the whole grid are plotted against the intrinsic time. The upper trace is from a simulation with $\sigma=0.667 \sigma^{*}$, whereas the lower trace is from one with $\sigma=0.9 \sigma^{*}$. (C) The scaled pinwheel densities are plotted against the intrinsic time, with a constant value of 4 observed in both simulations. 


\subsubsection{Dynamics in Large Systems}

Instead of a single orientation hypercolumn, the area V1 contains much more repetitive units. In other words, the dynamics of OPMs in the visual cortex resembles the behavior of a large system. Intriguingly, more complex patterns with a progressively longer period of dynamical evolution were observed in simulations of systems from $L=1 \Lambda_{\max }$ to $L=5 \Lambda_{\max }$.

We have shown above that for $L=1 \Lambda_{\max }$, the checkerboard pattern consisting of four pinwheels was fairly stable. However, we observed different behaviors of long-term rearrangement in larger systems (Figure 4.6). For all system sizes we investigated $\left(\sigma=0.9 \sigma^{*}, 0.667 \sigma^{*}\right.$, and $\left.0.3 \sigma^{*}\right)$, the initial pinwheel configurations were typically unstable, with orientation stripes taking over almost all pinwheel components. The number of pinwheels generally decreased in long term simulation.

Figure 4.6 shows typical maps at early and late stages generated in different regimes of the bifurcation diagram for different system sizes. Close to the threshold $\left(\sigma=0.9 \sigma^{*}\right)$, the initial growth rate of map formation is small, resulting in a large intrinsic time scale $\tau$. In this regime the map dynamics converged fast to a checkerboard pattern in the small system of $L=1 \Lambda_{\max }$ and a stripe pattern in the larger systems. The 'final' patterns of stripes seemed rather stable after the first $50 \tau$. The time scale for map convergence was substantially longer for systems far from threshold. In these simulations we had to follow hundreds of intrinsic time scales in order to track the pattern rearrangement after the initial map establishment.

Another general behavior of the map dynamics is a reduction of the number of repetitive units. In systems far from threshold $\left(\sigma \ll \sigma^{*}\right)$, the spectrum of positive eigenvalues is broader then near threshold (see Figure 2.2). This apparently enables a process called domain coarsening (Figure 4.6C). The significant domain coarsening in systems of sizes $L=2 \sim 3 \Lambda_{\text {max }}$ might be influenced by the periodic boundary condition. However, the phenomenon of domain coarsening was also found in simulations with fixed boundary condition (see Figure 1.4), which seemed unlikely to be an artifact of the boundary effect.

To understand why the system size can induce qualitative different behaviors, it is instructive to consider a mechanical analog. Imagine there are four balls of different colors in a box (Figure 4.7). If the average spacing between two balls is smaller than the radius of an individual ball, it is impossible to move a ball from one side to another. Thus, the relative positions of each ball will not change. Now let's consider four repetitions of such units in the box, although the spacing and the radius of each ball remain the same, we can easily move one ball to another side and reorganize the system. It is not surprising that dynamical change and pattern reorganization are feasible in larger systems. 

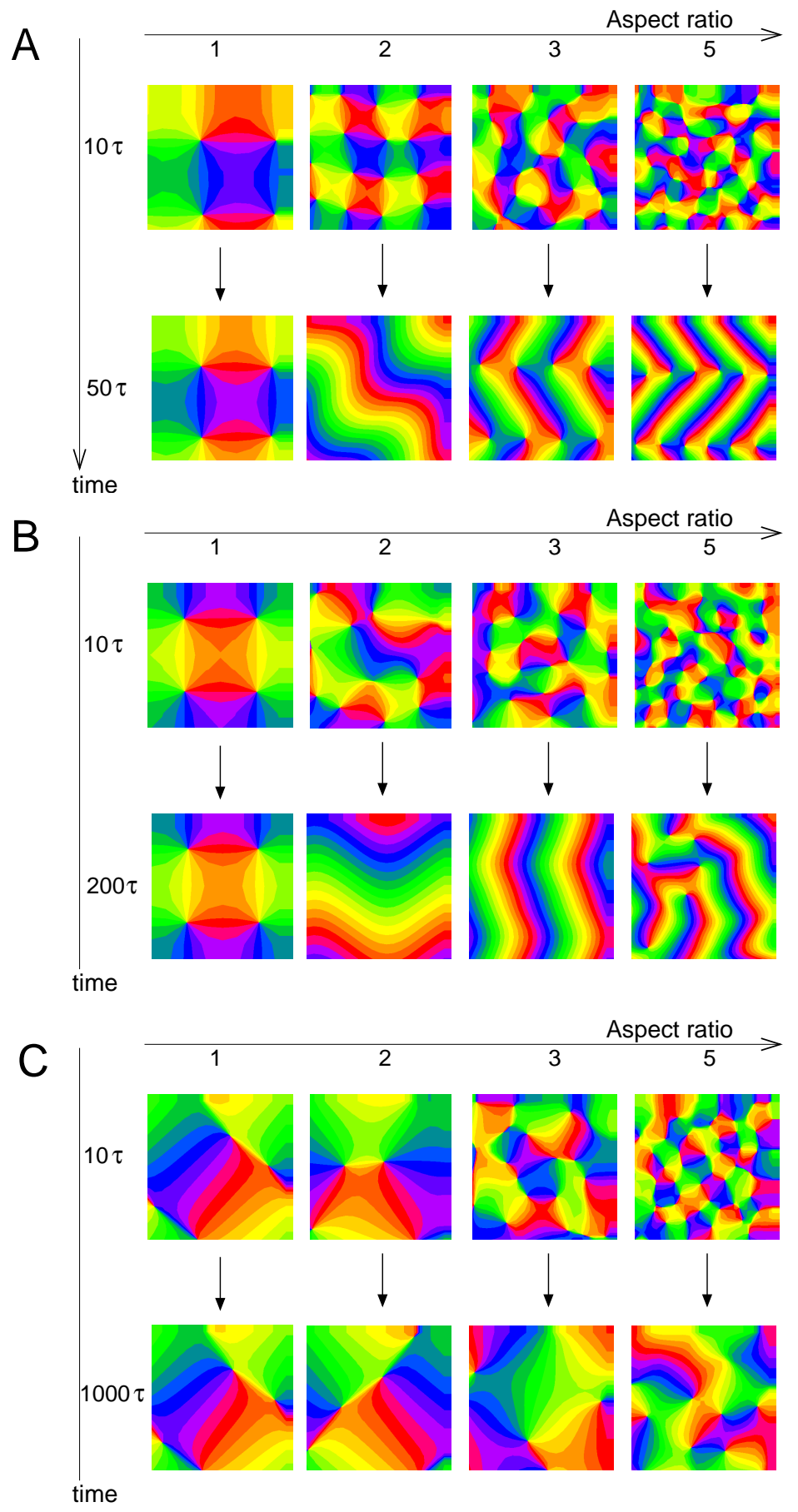

Figure 4.6: System size dependence of OPM dynamics $(2 \mathrm{D}+2 \mathrm{D})(\mathrm{A})$ $\sigma=0.9 \sigma^{*}$; (B) $\sigma=0.667 \sigma^{*}$; (C) $\sigma=0.3 \sigma^{*}$. The upper row shows representative patterns at $t=10 \tau$ for $L=1 \Lambda_{\max }, 2 \Lambda_{\max }, 3 \Lambda_{\max }$, and $5 \Lambda_{\max }$, respectively. The bottom row shows results from the same simulation but at $(\mathrm{A}) t=50 \tau$, (B) $t=200 \tau$ and $(\mathrm{C}) t=1000 \tau$. 


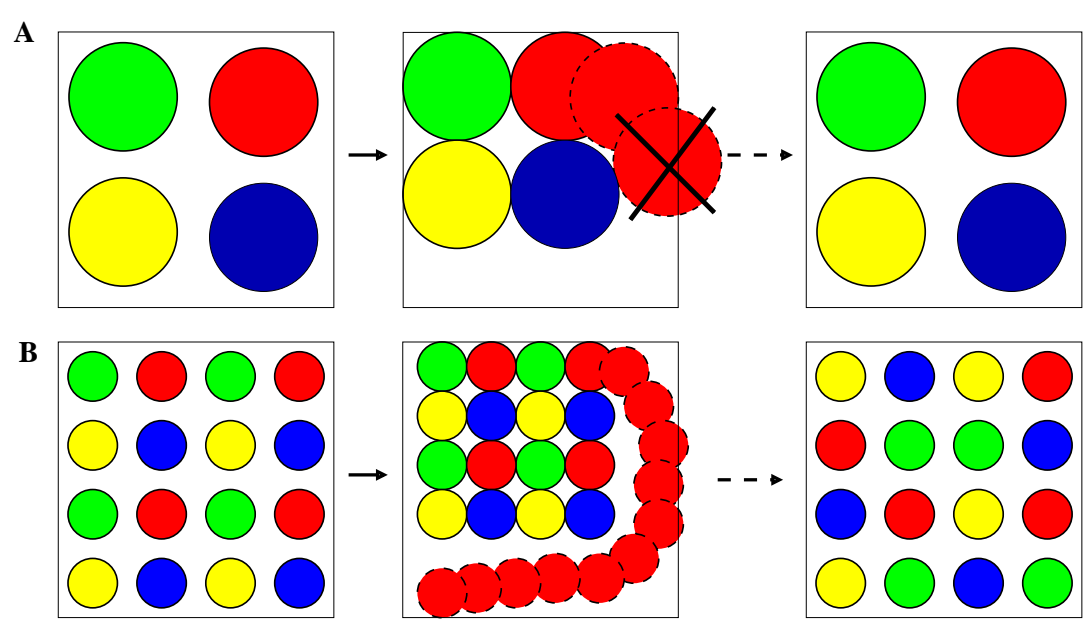

Figure 4.7: Mechanical analog: the system size effect (A) No reorganization can happen in the small box which is tightly packed by four balls of different colors. (B) In a big box consisting of four repetitive units as in (A), one can easily move the balls to get a new pattern of different colors.

\subsection{The Kinetics of Pinwheel Annihilation}

We quantified the kinetics of pinwheel annihilation in the Kohonen model (Figure 4.8) by the number of pinwheels $n_{\mathrm{pw}}$, the average column spacing $\Lambda$, and the scaled pinwheel density $\rho \Lambda^{2}$ (for a description of methods see Chapter 3).

In simulations, we observed generations of pinwheels from the initially homogenous state, which was followed by a second phase of pinwheel annihilation with a dramatic reduction of pinwheel numbers (Figure 4.8 A,B). The first phase of pattern growth was rather fast in systems far from threshold $\left(\sigma=0.667 \sigma^{*}\right)$ such that only the second phase is visible in Figure 4.8B. The size of a hypercolumn is measured by the average column spacing $\Lambda$ of the pattern (Figure 4.8C,D), while the scaled pinwheel density $\rho \Lambda^{2}$ is a dimensionless quantity that measures the average number of pinwheels in an area of size $\Lambda^{2}$ (Figure 4.8E,F). Except for $L=1 \Lambda_{\max }$, in which the scaled density was always 4 , the other systems with more than one column spacing gradually lost pinwheels and the final pinwheel densities were generally below 2 .

In addition, there seems to be a limit curve in the plot of the scaled densities. The kinetics for $L=4 \Lambda_{\max }$ and for $L=5 \Lambda_{\max }$ became indistinguishable in Figure 4.8. This indicates that systems of size $4 \Lambda_{\max }$ or $5 \Lambda_{\max }$ are presumably representative of the larger system behavior. 

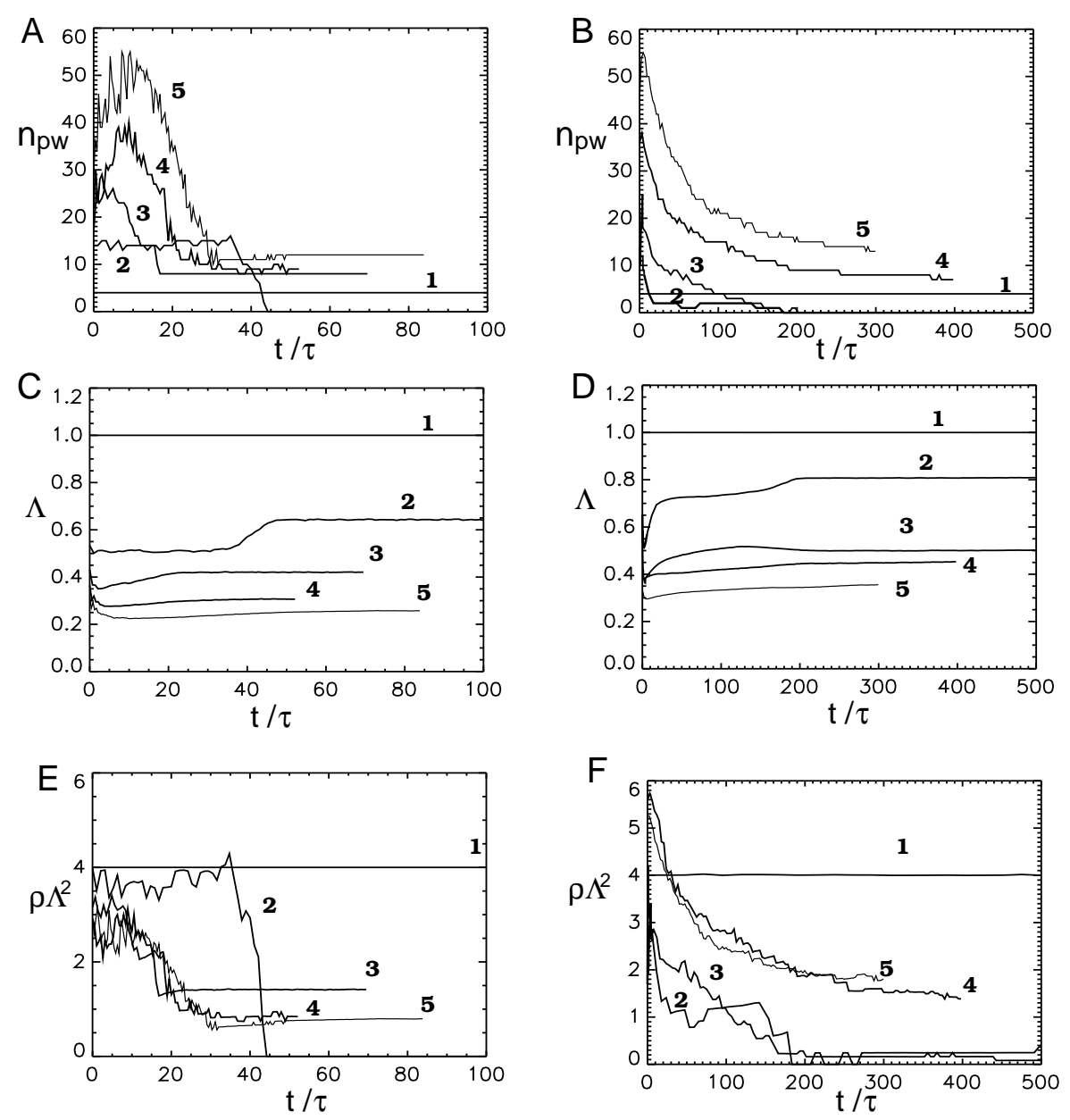

Figure 4.8: The Kinetics of pinwheel annihilation for $\sigma=0.9 \sigma^{*}$ (left column) and $\sigma=0.667 \sigma^{*}$ (right column) (A,B) Pinwheel numbers counted over the entire model cortex. (C,D) The average column spacing and (E,F) the scaled pinwheel density. Each trace shows the average value for three different initializations of the random stimulus generator. Numbers on the trace indicate the aspect ratio $\left(L / \Lambda_{\max }\right)$ of the systems. Time is measured in units of $\tau$, the intrinsic timescale of the initial symmetry breaking. 


\subsection{Summary}

In this chapter we first demonstrated the spontaneous map formation in simulations at a critical point of $\sigma$, whose value is in consistence with the theoretical prediction. A bifurcation diagram was constructed such that the subsequent simulations could be performed close to or far away from the critical bifurcation point. We simulated the map dynamics over durations much longer than the intrinsic time scale of initial pattern formation. Presenting only a small set of fixed stimuli was found to stop the temporal evolution of OPMs. Then we characterized the model behavior in systems of different sizes. Small systems of one hypercolumn $\left(L=1 \Lambda_{\max }\right)$ exhibited a stable checkerboard pattern containing four pinwheels. However, the map dynamics became unstable when we increased the system size from $L=1 \Lambda_{\max }$ to $L=5 \Lambda_{\max }$. In the large systems, pinwheels collided and annihilated with each other. Throughout the parameter regimes we investigated $\left(\sigma=0.9 \sigma^{*}, 0.667 \sigma^{*}\right.$, and $\left.0.3 \sigma^{*}\right)$, the initial pinwheel-rich patterns were replaced by orientation stripes in long term simulations.

Simulation time was measured in units of the intrinsic time scale $\tau$ of pattern formation. The dynamics of systems closed to the critical bifurcation point were comparatively faster than those far below the critical threshold. Generally, substantial pattern reorganization took place during a period on the order of $10 \tau$.

The kinetics of pinwheel was quantified by the pinwheel density, which measures the average numbers of pinwheel per hypercolumn. The scaled pinwheel density remained 4 in small systems $\left(L=1 \Lambda_{\max }\right)$ but decayed to a value below 2 in all larger systems. As a comparison, a statistical quantification of OPMs in different species such as tree shrew, ferret and galago has recently suggested that the pinwheel density is around 3.14 per hvpercolumn (Kaschube, 2005: Kaschube et al.,2006). Our results demonstrated that such a pinwheel-rich pattern is largely unstable in mappings of orientation preference to model cortex by competitive Hebbian learning. 


\section{Chapter 5}

\section{Map Dynamics with Higher Dimensional Feature Spaces}

So far, the models only mapped retinotopy and orientation $(2 \mathrm{D}+2 \mathrm{D})$ to the visual cortex. However, at a given visual field location, all possible features of orientations, spatial frequencies, and binocular visual cues should be represented in the corresponding cortical area. Effectively, the constraint of coverage uniformity might partition the entire cortex to small areas in which pinwheel dynamics might be stabilized as in the $\Gamma=1$ systems discussed above. For example, pinwheels have often been observed in the center regions of the ocular dominance columns (Crair et al., 1997b; Hübener et al., 1997).

In this chapter, we thus include more feature dimensions in the model to test the hypothesis that interactions of different feature maps will stabilize pinwheel patterns in OPMs. The resulting feature space consists of two retinal dimensions plus $n$ feature dimensions $(2 \mathrm{D}+\mathrm{nD})$. For simplicity, we assumed an isotropic distribution of visual stimuli in the $n$-dimensional feature space and constructed orientation preference maps from combinations of every two feature dimensions (for methods see Chapter 31).

\subsection{Dimension Suppression Close to Symmetry- breaking Threshold}

To our surprise, dimension suppression was observed in simulations close to the critical point of pattern formation $\left(\sigma=0.9 \sigma^{*}\right)$ such that only two feature dimensions emerged at the symmetry breaking threshold; the other feature dimensions were suppressed until a secondary bifurcation point.

First, we added one more feature dimension to the system, to consider the mapping from $2 \mathrm{D}+3 \mathrm{D}$ feature space (e.g., two dimensions for retinotopy, two dimensions for orientation, and one additional dimension for ocular dominance).

Close to the symmetry breaking threshold $\left(\sigma=0.9 \sigma^{*}\right)$, only two feature 


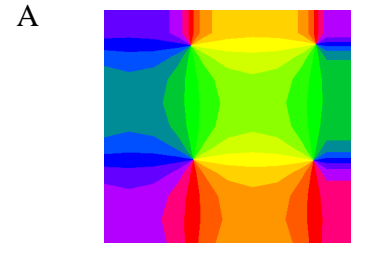

B

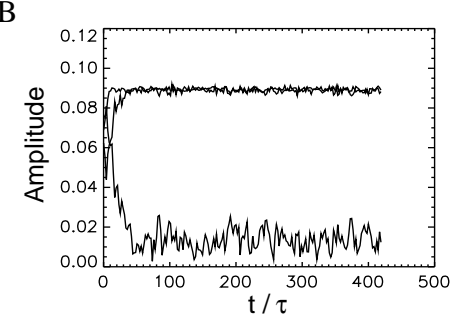

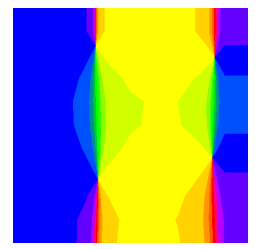
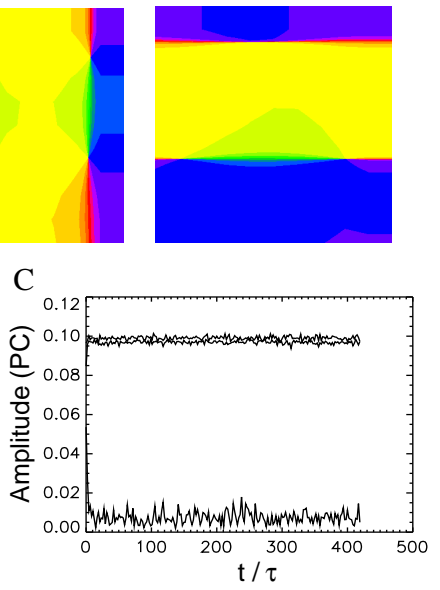

Figure 5.1: Dimension suppression in small systems $(2 \mathrm{D}+3 \mathrm{D}$ feature space) $\sigma=0.9 \sigma^{*}, L=1 \Lambda_{\max }$. (A) OPMs generated with combination of every two feature components. (B) The amplitude for three feature dimensions averaged over the entire model cortex. (C) The average amplitude for the three principle components obtained by PCA.

dimensions were virtually active in simulations. We observed spontaneous separation of the three feature dimensions to the first two with larger amplitudes (Figure 5.1B, upper two traces) and another one with a smaller amplitude (Figure $5.1 \mathrm{~B}$, lower two traces).

The patterns of OPMs shown in Figure 5.1A were generated by mapping of the half angle of a $2 \mathrm{D}$ feature vector to its corresponding cortical location $\theta(\mathbf{x})=0.5 \arg \left(\mathrm{z}_{\mathrm{i}}+\mathrm{i} \mathrm{z}_{\mathrm{j}}\right)$. Only the combination of the two feature components $z_{i}$ and $z_{j}$ with largest amplitude showed a stable pattern of four pinwheels (Figure 5.11A, left panel). In other combinations, the resulting orientation maps exhibit abrupt transitions from a square patch of one orientation to another patch of orthogonal orientation (Figure 5.1A, middle and right panels). Our interpretation is that the map degenerates to one axis in these regions with positive or negative values corresponding to two orthogonal orientations.

To rule out the possibility that the breaking of rotational symmetry is due to the computer round-off error accumulating on the main axes, we performed simulations with the same parameters, but rotated the coordinate system every $\tau / 2$. Principle component analysis (PCA) of the data showed a clear separation of represented and suppressed feature space dimensions (Figure [5.1C), which suggested the tendency for the distribution of the feature vectors to collapse onto a 2D submanifold of the high-dimensional feature space.

Next, we systematically changed the bifurcation parameter $\sigma$ to explore the dynamics of a high dimensional feature maps. In principle, we can use the same method as that presented in Figure 4.2 to obtain the bifurcation diagram. For 


\subsection{Dimension Suppression Close to Symmetry-breaking Threshold 43}

A

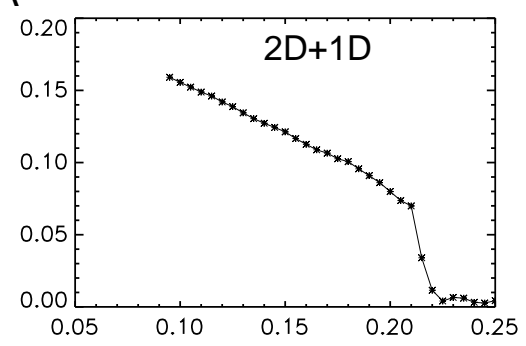

C

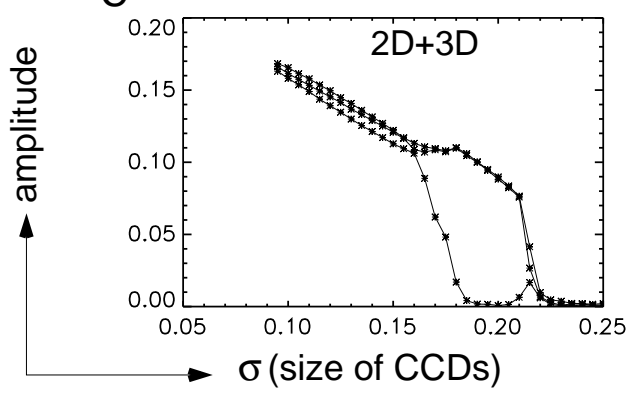

$\mathrm{B}$

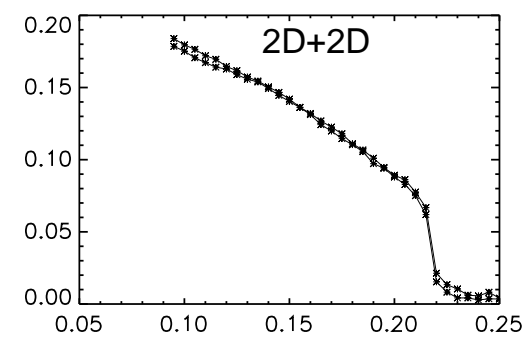

D

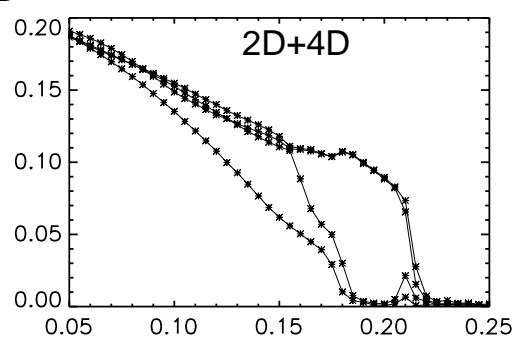

Figure 5.2: Bifurcation diagrams (Gaussian distributions) The stimulus variance $\sigma_{s}=0.25$ whereas the size of co-activated cortical domains varied subsequently in one simulation. The amplitude of the principal component measures the greatest variance by any projection of the data set; when the values are significantly above 0 , it indicate pattern emergence in the feature space. (A) Maps from 2D $+1 \mathrm{D}$ feature space; (B) Maps from 2D $+2 \mathrm{D}$ feature space (C) Maps from $2 \mathrm{D}+3 \mathrm{D}$ feature space; (D) Maps from $2 \mathrm{D}+4 \mathrm{D}$ feature space. The first two dimensions for the retinotopy; the amplitudes of additional feature dimensions are plotted here. 
computational efficiency, we performed one simulation with a fixed value of stimulus variance $\sigma_{s}=0.25$ while reducing the size of CCDs by 0.001 after each intrinsic time scale of $1 \tau$. The bifurcation diagrams shown in Figure 5.2 plot at each time point the largest variance of the feature vector distribution as revealed by PCA. In each panel, different feature spaces of different dimensionalities are mapped to the model cortex, increasingly from $2 \mathrm{D}+1 \mathrm{D}$ to $2 \mathrm{D}+4 \mathrm{D}$. As predicted by the linear stability analysis, the pattern spontaneously emerged at the first bifurcation point, around the value of $\sigma^{*}=0.215$, irrespective of feature spaces dimensionality. For feature space dimension larger than two, a secondary bifurcation was observed at $\sigma^{* *}=0.180$. A value of $\sigma^{* *}<\sigma<\sigma^{*}$ resulted in only two active feature dimensions. All other feature dimensions were suppressed and only started to grow if we further decreased the value of $\sigma$ below the secondary bifurcation point $\sigma^{* *}$.

\section{Map Dynamics in the Dimension Suppression Regime}

Figure 5.3 shows representative patterns of orientation maps with $\sigma=0.9 \sigma^{*}$ generated with $2 \mathrm{D}+2 \mathrm{D}, 2 \mathrm{D}+3 \mathrm{D}$ and $2 \mathrm{D}+4 \mathrm{D}$ feature spaces respectively. Maps at early stages of initial pattern establishment are compared with those at later stages. In these simulations, pinwheel structures were again unstable for all combinations of different feature components. The final pattern was typically a mixture of stripes and square patches. There are only two colors dominating the regions of a square patch alternatively, which implies that one feature dimension is suppressed locally. Orientation stripes represent regions in which both feature dimensions are active.

\subsection{Pinwheel Annihilation for Low Dimensional Feature Spaces}

Due to dimension suppression close to the symmetry breaking threshold (for $\sigma^{* *}<$ $\sigma<\sigma^{*}$ ), the model maps failed to encode more than two feature dimensions. The behavior is thus expected to be similar to the $2 \mathrm{D}$ case as previously described. Hence we are more interested in the parameter regimes far from the symmetry breaking threshold where all the feature dimensions are active. In the following we show simulations of the systems one-third below the threshold $\left(\sigma=0.667 \sigma^{*}<\right.$ $\left.\sigma^{* *}\right)$.

In the model cortex mapping from $2 \mathrm{D}+3 \mathrm{D}$ feature space, different combinations of feature components generate similar patterns of OPMs at the initial phase of pattern growth (Figure 5.4A, upper row). Yet, all the maps lost pinwheel singularities in long term simulation (Figure 5.4A, bottom row). The number of pinwheels reduces to less than one third at $t<200 \tau$ (Figure 5.4B), a time scale 


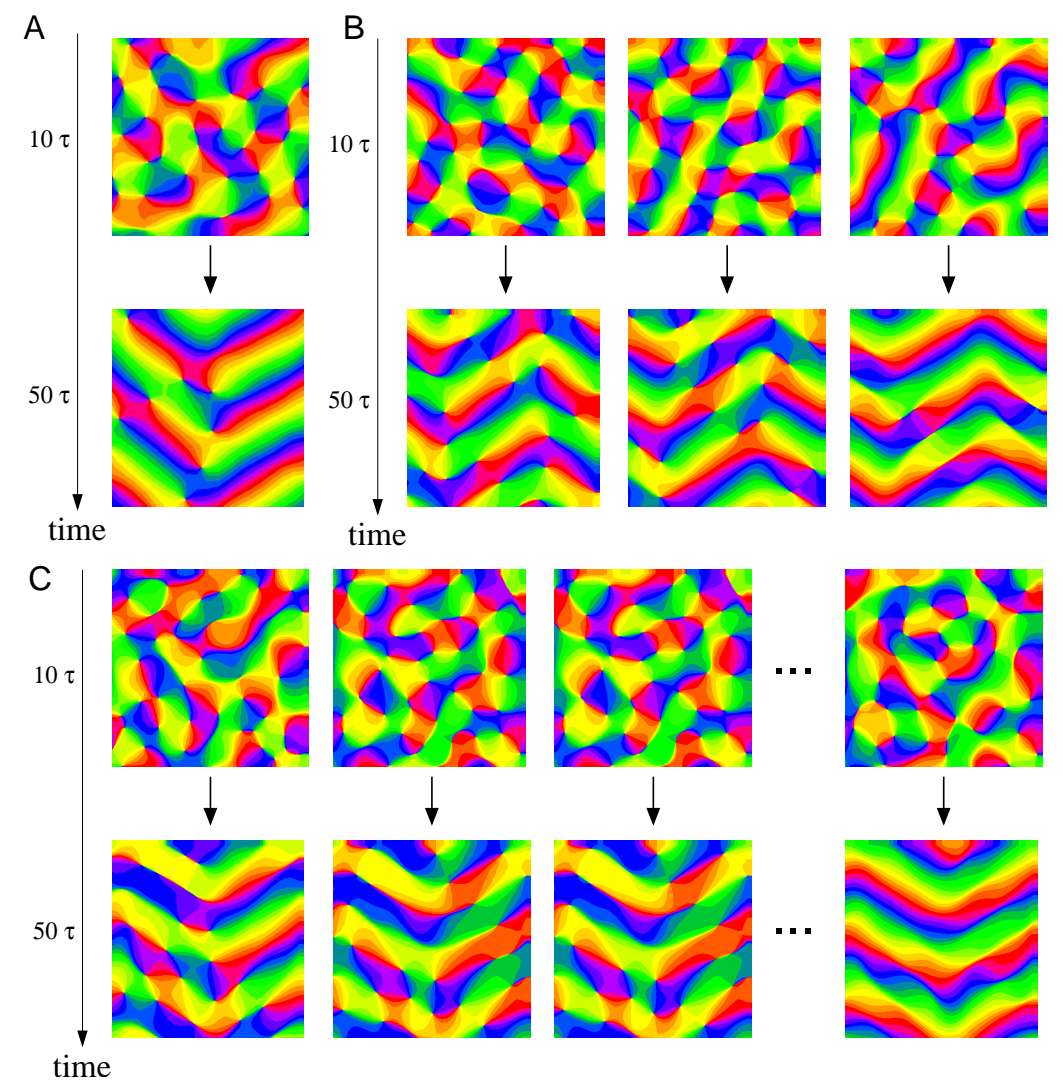

Figure 5.3: Example maps of different feature dimensions ( $\left.\sigma=0.9 \sigma^{*}\right)$ The orientation preference maps generated at an early stage $(t=10 \tau)$ contained a large number of pinwheels. The rearrangement of each map removed most of the pinwheels at $t=50 \tau$. Qualitatively similar patterns were observed for (A) the $2 \mathrm{D}+2 \mathrm{D}$ model, $(\mathrm{B})$ the $2 \mathrm{D}+3 \mathrm{D}$ model and $(\mathrm{C})$ the $2 \mathrm{D}+4 \mathrm{D}$ model. 

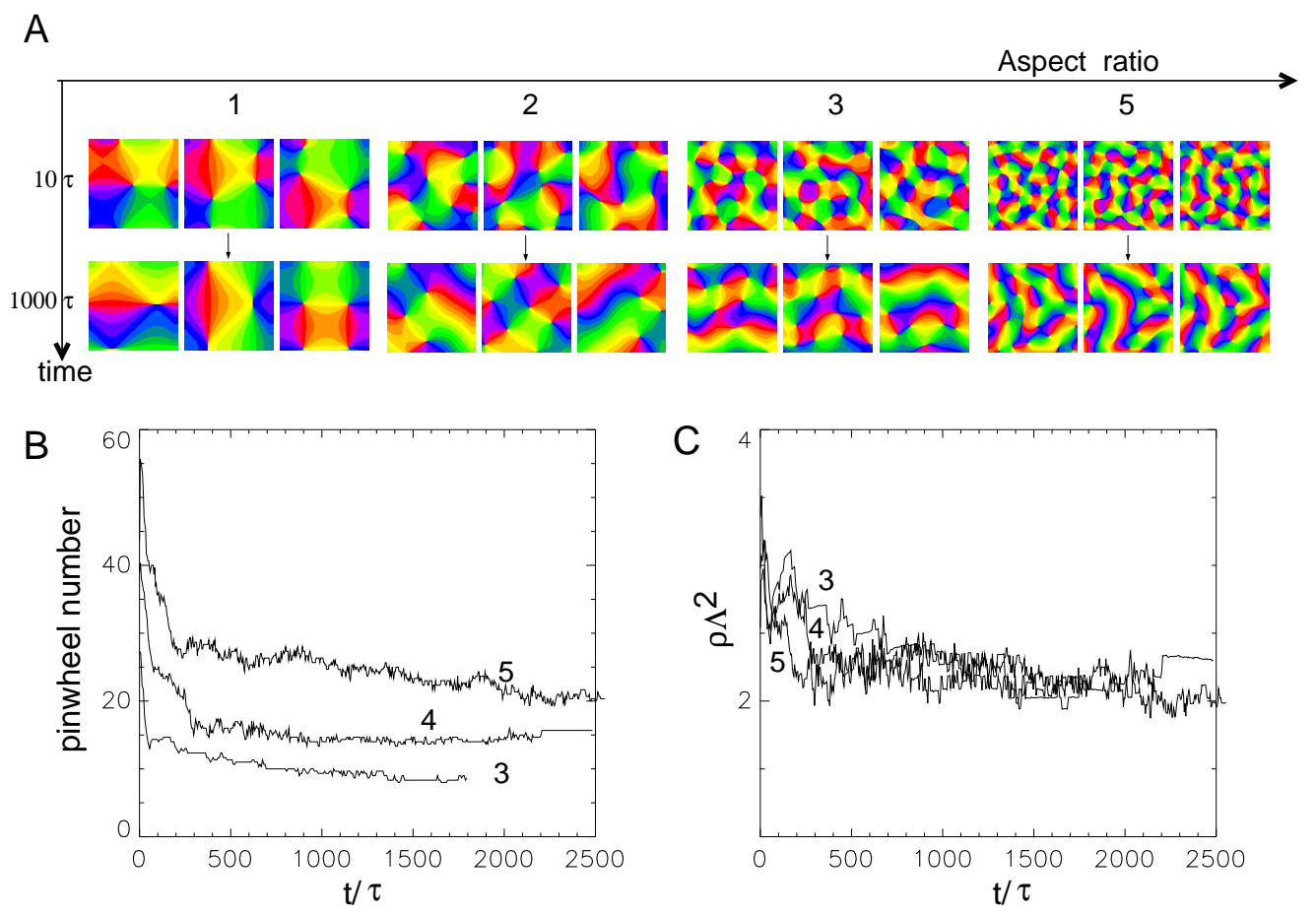

Figure 5.4: Pinwheel dynamics $\left(2 \mathrm{D}+3 \mathrm{D}\right.$ feature space, $\left.\sigma=0.667 \sigma^{*}\right)(\mathrm{A})$ Maps generated in systems of $L=1 \Lambda_{\max }, 2 \Lambda_{\max }, 3 \Lambda_{\max }$ and $L=5 \Lambda_{\max }$, at $t=10 \tau$ (upper row) and $t=1000 \tau$ (bottom row). (B) The number of pinwheels averaged over three maps. (C) The scaled pinwheel density averaged over three maps. Numbers indicate aspect ratio of the system. 
of pinwheel annihilation comparable to the simulations of uncoupled OPMs (Figure 4.8). However, the scaled pinwheel density plotted in Figure 5.4C decays less strongly, which might be due to more prominent domain coarsening with a broader spectrum of positive eigenvalues. The increasing column spacing counteracts the reduction of pinwheel numbers in the measure of pinwheel density per hypercolumn. Above all, the scaled density falls below two, which suggests the development of OPMs coupled to one additional feature (e.g., ocular dominance) cannot maintain a large number of pinwheels in the Kohonen model.

\subsection{Pinwheel Crystallization for High Dimensional Feature Spaces}

Intriguingly, while mapping from $2 \mathrm{D}+4 \mathrm{D}$ feature space, four feature dimensions spontaneously fall into two pairs. The final pattern can be described as two plane waves in the four-dimensional feature space. Figure 5.5A shows the six patterns generated in the system of size $L=4 \Lambda_{\max }$. Combinations within a pair generate maps of stripes, whereas inter-pair combinations produce patterns of pinwheel crystals. In the former case, the dynamics of OPMs undergoes a continual process of pinwheel annihilation until almost all the singularities vanish; however, in the latter case, the initial drop-down of pinwheel numbers stopped and was followed by a regeneration and stabilization of pinwheel crystals (Figure 5.5B). The scaled density reaches either zero or a value close to 4 (Figure 5.5C).

Similar behaviors were found in different sizes of systems (Figure 5.3). In contrast to the large variability of map layouts in experimentally observed OPMs, the pinwheel crystals show a regular structure with a constant wavelength in different sub-regions of the map. In systems larger than $3 \Lambda \times 3 \Lambda$, we found that patterns of stripes and pinwheel crystals intermingle in one map.

We tested whether the separation of feature dimensions and the formation of pinwheel crystals resulted from mappings of an even dimensional feature space (Figure 5.5) compared with an odd dimensionality in 2D $+3 \mathrm{D}$ models (Figure 5.4). They turned out to be a general property of high dimensional feature spaces of $n>3$. Figure 5.3 plots maps generated in mappings of $2 \mathrm{D}+5 \mathrm{D}$ feature spaces. The initial pinwheel-rich patterns are replaced by either stripes or crystals in different combinations of feature dimensions.

\subsection{Summary}

In the mappings of a high-dimensional feature space model, we found that close to the critical point of pattern formation, only two feature dimensions grew from the initially unselective state whereas the additional feature dimensions were suppressed. The model behaved as if there was only two relevant feature dimensions, 

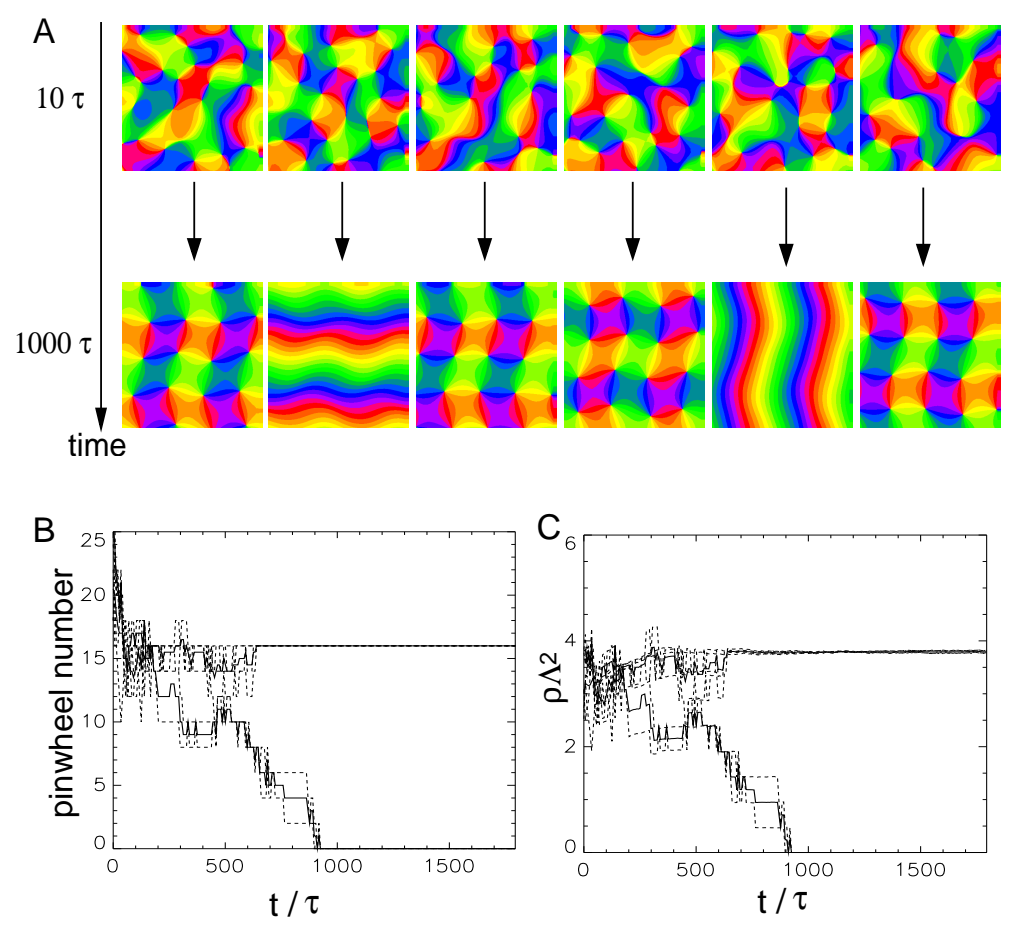

Figure 5.5: Pinwheel dynamics $\left(2 \mathrm{D}+4 \mathrm{D}, \sigma=0.667 \sigma^{*}, L=4 \Lambda_{\max }\right)(\mathrm{A})$ The six patterns of OPMs generated by each pair of feature dimensions. The top row depicts maps generated at $t=10 \tau$; The bottom row shows the pattern of each map at $t=1000 \tau$. (B) The number of pinwheels plotted in time. (C) The scaled pinwheel density. Dashed line: individual maps; solid line: the average of three maps. Time is weighted by the intrinsic timescale $\tau$. 


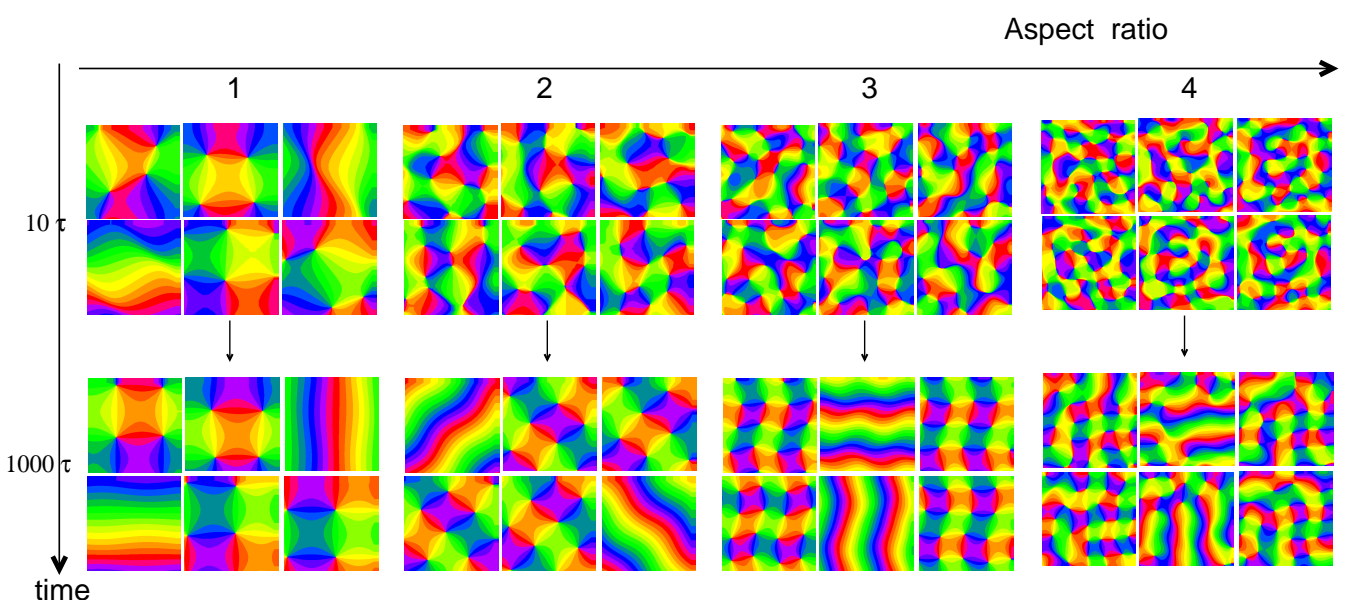

Figure 5.6: Pinwheel crystallization $\left(2 \mathrm{D}+4 \mathrm{D}\right.$ feature space, $\left.\sigma=0.667 \sigma^{*}\right)$ The upper row shows the maps generated at $t=10 \tau$ for $L=1 \Lambda_{\max }, 2 \Lambda_{\max }, 3 \Lambda_{\max }$, and $4 \Lambda_{\max }$, respectively. The bottom row shows the patterns from the corresponding simulations as the first row, but at $t=1000 \tau$.

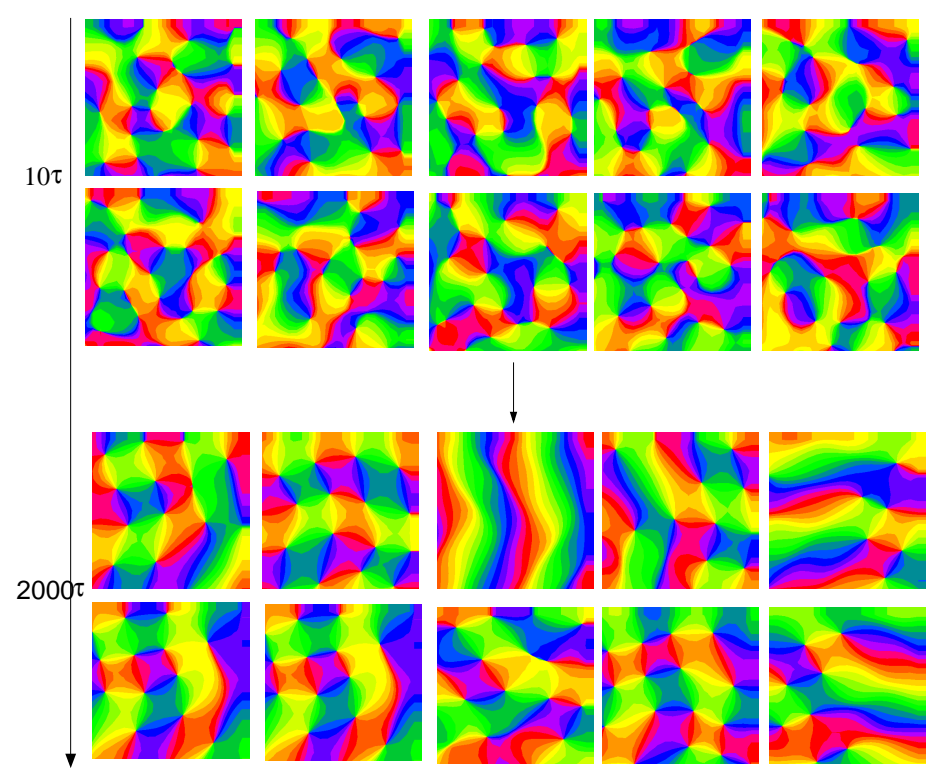

Figure 5.7: Pinwheel Crystallization $\left(2 \mathrm{D}+5 \mathrm{D}\right.$ feature space, $\left.\sigma=0.667 \sigma^{*}\right)$ The upper row shows the maps generated at $t=10 \tau$ for $3 \Lambda_{\max }$. The bottom row shows the corresponding patterns from the same simulations, but at $t=2000 \tau$. 
and thus showed an unstable pinwheel dynamics as the 2D systems irrespective of the feature space dimensionality. When we further reduce the size $\sigma$ of coactivated cortical domains, a sequence of bifurcations occur such that more and more feature dimensions become active.

In this regime far from threshold, we observed pinwheel annihilation in lower dimensional feature spaces and pinwheel crystallization in higher dimensional feature spaces. The results revealed the development of OPMs as a dynamical process where different feature dimensions select either pinwheel-free stripes or pinwheel crystals in the final patterns. Both of them do not resemble the observed patterns of OPMs in the visual cortex. 


\section{Chapter 6}

\section{Map Dynamics with Non-Gaussian Stimulus Distributions}

Since the model cortex updates the feature values of its receptive field based on the Hebbian plasticity of input-output correlations, another factor that needs to be considered is the type of stimulus distribution. Besides Gaussian statistics, we thus used two other types of stimulus distribution which are naturally associated with different feature spaces.

For the model with a spherical feature space, we picked stimuli from uniform distribution on a n-dimensional sphere of radius $\sqrt{2} \sigma_{s}$. To distinguish from the Gaussian feature space, we used $S^{n}$ to specify the feature space on an ndimensional sphere, so that $S^{2}$ represented a $2 \mathrm{D}$ sphere in a $3 \mathrm{D}$ space whereas $S^{3}$ indicated a $3 \mathrm{D}$ sphere in a $4 \mathrm{D}$ space.

Another model with toroidal feature space comprised products of circular variables (such as orientation and direction). The values of feature vectors were grouped into $n / 2$ pairs; each pair was used to define an angular variable indicating the preferred orientation, which was distributed uniformly on the circle of radius $\sqrt{2} \sigma_{s}$. We denote this feature space as $S^{1} \times S^{1} \times \ldots \times S^{1}$ where one circle $S^{1}$ represents one circular variable.

\subsection{Models with a Spherical Feature Space}

In models with a spherical feature space, we assumed that the feature vector of each stimulus had identical length; only its orientation in the feature space should determine the activated winner position in the cortex.

Figure 6.1 plots the amplitudes of individual feature components while decreasing the size $\sigma$ of the co-activated cortical domains subsequently in one simulation. The bifurcation diagrams are quantitatively similar to those from $2 \mathrm{D}+3 \mathrm{D}$ and $2 \mathrm{D}+4 \mathrm{D}$ models with Gaussian distributed stimuli (Figure 5.2C,D). When decreasing the size $\sigma$ of coactivated domains, patterns emerged at the first bifur- 
cation point $\sigma^{*}=0.215$. There were however only two active feature dimensions until the secondary bifurcation point $\sigma^{* *}=0.180$ was reached.

Linear stability analysis around the homogenous state has deduced the first symmetry breaking threshold $\sigma^{*}=\sqrt{2 / e} \sigma_{s}$ (see Chapter 21), irrespective of the feature space dimensionality and the type of stimulus distribution. When comparing simulations of the spherical feature space model with those of the Gaussian feature space model, it seemed that the second bifurcation point $\sigma^{* *}$ was also invariant to the details of the stimulus distribution in the feature space.
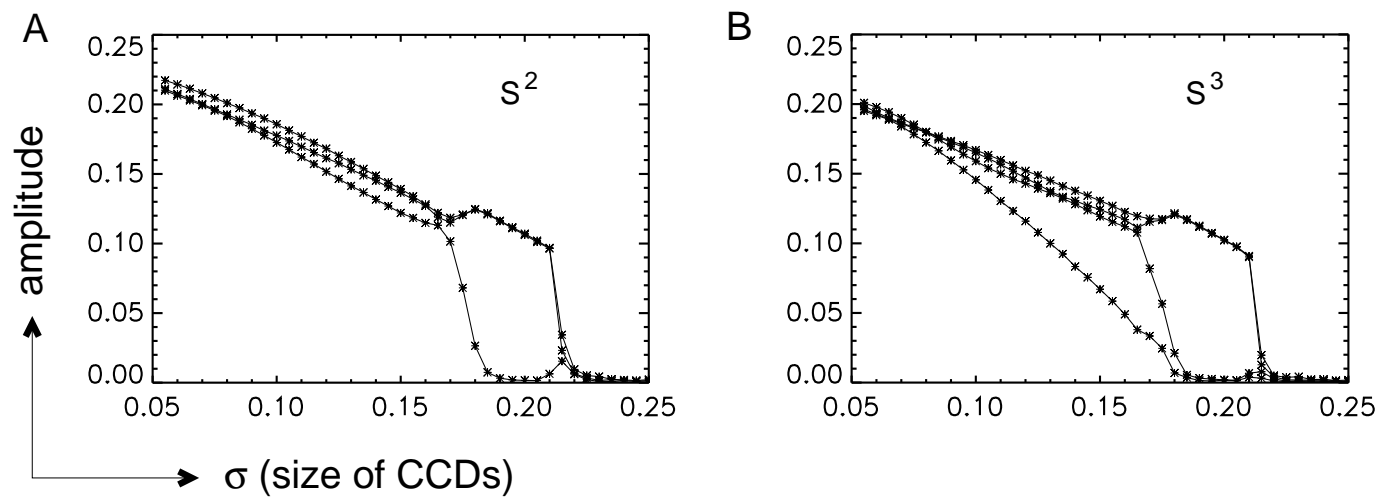

Figure 6.1: Bifurcation diagrams for the spherical model (A) Stimuli were drawn from the uniform distribution on $S^{2}$. The amplitudes of three individual feature components plotted against the size $\sigma$ of CCDs. (B) Stimuli were drawn from uniform distribution on $S^{3}$. The amplitudes of four individual feature components plotted against the size $\sigma$ of CCDs.

The dynamics of orientation maps in the model with spherical feature space was studied in different regimes of the bifurcation diagram. The unstable pinwheel dynamics were qualitatively similar compared to those in the Gaussian feature spaces (see Chapter 4)

In Figure 6.2, we show a simulation of the $S^{2}$ model with $\sigma=0.667 \sigma^{*}$ far from the threshold so that all feature dimensions were active. Few pinwheels were preserved in the long term. The number of pinwheels dropped to one third of the initial value at $t<100 \tau$ (Figure 6.2 B). The process of pinwheel annihilation was faster than with Gaussian distributed stimuli. The domain coarsening was more prominent in the sense that the average column spacing increased significantly in time. Hence, we observed less decrease of the scaled pinwheel density $\rho \Lambda^{2}$ in the $S^{2}$ feature space model. 

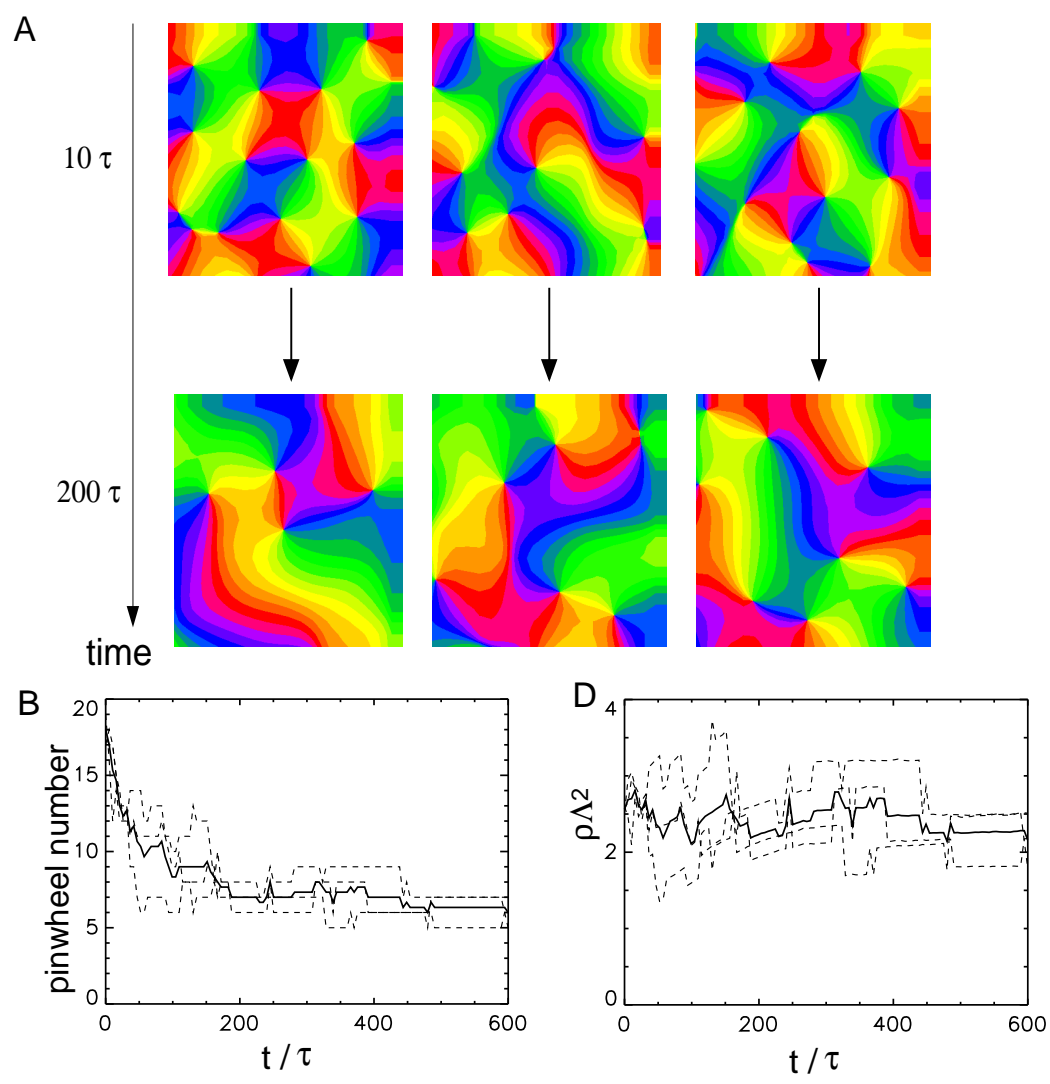

Figure 6.2: Pinwheel dynamics in model of $S^{2}$ feature space, $\sigma=0.667 \sigma^{*}$ (A) Representative orientation preference maps at early and late stages of one simulation. The top row depicts three maps generated at $t=10 \tau$; The bottom row shows the pattern of each map at $t=200 \tau$. (B) The number of pinwheels over simulation time. (C) The scaled pinwheel density. Time is measured in the unit of the intrinsic timescale $\tau$. Dashed line: individual maps; solid line: the average of three maps. 


\subsection{Models with a Toroidal Feature Space}

Figure 6.3 plots the bifurcation diagrams for the toroidal model. The behavior was similar to that of former models with other stimulus distributions, with the first bifurcation point around $\sigma^{*}=0.215$. The value of the secondary bifurcation point varied with the feature space dimensionalities (see Figure 6.3).

A
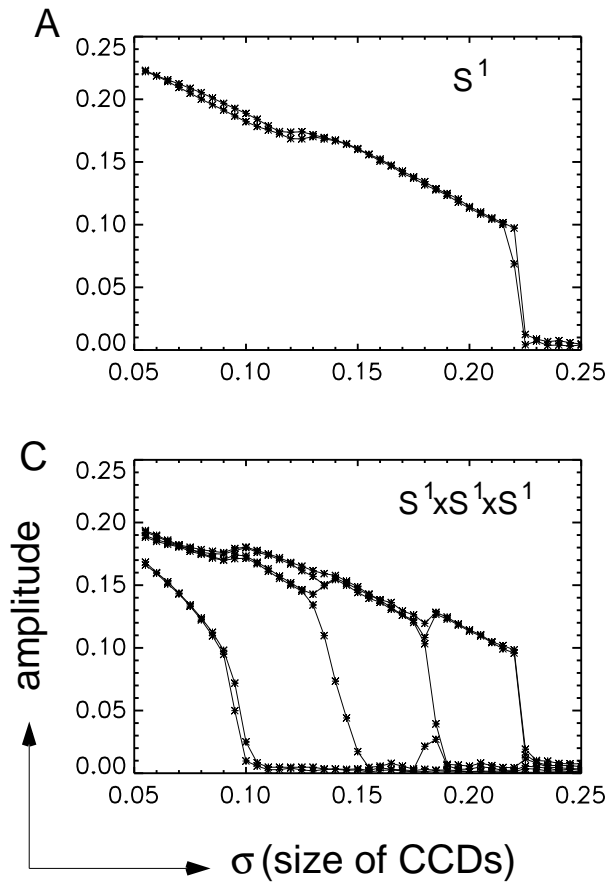

$\mathrm{B}$

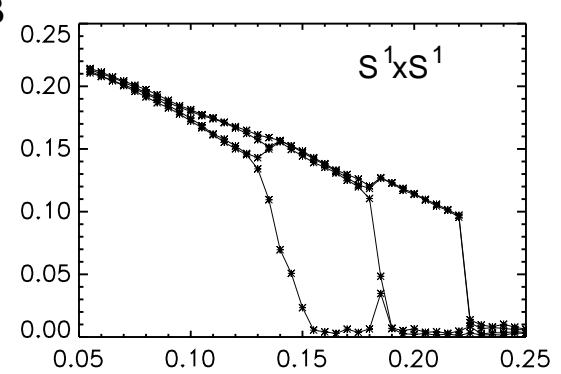

D

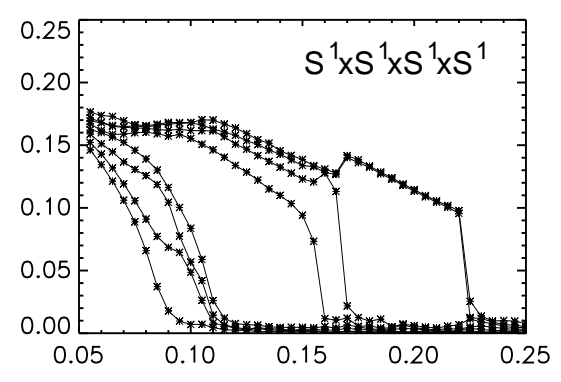

Figure 6.3: Bifurcation diagrams for the toroidal models The feature space comprises products of circular variables. Stimuli were drawn from the uniform distribution on each circle. The amplitudes of individual feature components are plotted against the size $\sigma$ of CCDs. The corresponding number of feature components is $2,4,6,8$ in $(A),(B),(C),(D)$, respectively.

The orientation preference maps generated in the $S^{1} \times S^{1}$ with arbitrary combinations of two feature dimensions are depicted in Figure 6.4. Close to the symmetry breaking threshold $\left(\sigma=0.9 \sigma^{*}\right)$, only two feature dimensions were active. The obtained patterns of OPMs were similar to those from mappings of Gaussian feature spaces. Pinwheels annihilated with each other even faster compared to Gaussian stimulus distributions. In later stages, the maps exhibited regions with large patches of one dominant orientation. The regions representing all orientations had a stripe-like organization of orientation domains. Although the feature values of the stimuli were set in two pairs, the final patterns of orientation stripes could also emerge from inter-pair combinations (Figure 6.4A, bottom 
panel), which suggested that the model might not treat the circular variable of two components as one parameter of the vector angles.
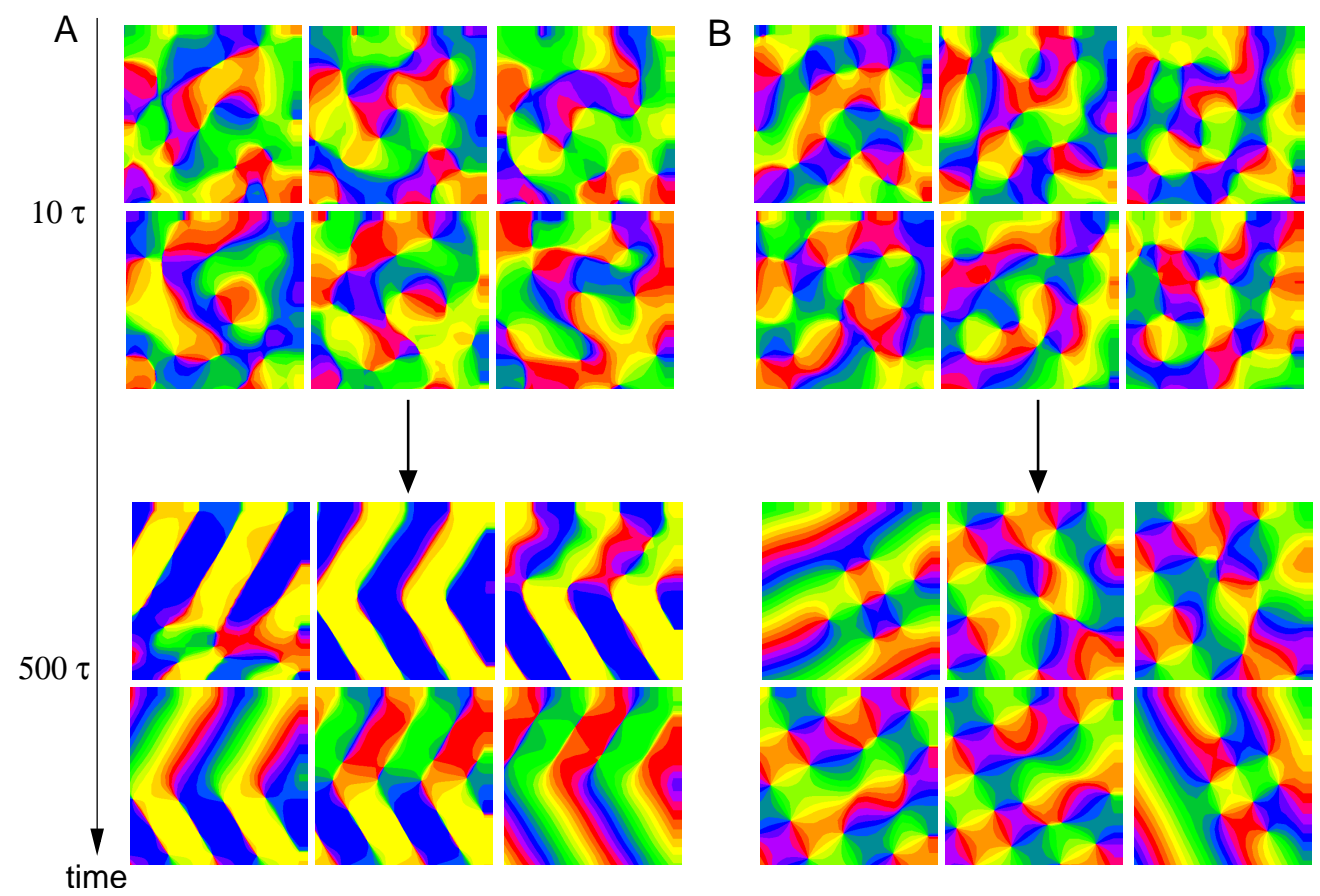

Figure 6.4: Maps generated form $S^{1} \times S^{1}$ feature space (A) The orientation maps generated with $\sigma=0.9 \sigma^{*}$. (B) The orientation maps generated with $\sigma=$ $0.667 \sigma^{*}$. The top panel is from simulation at an early stage $(t=10 \tau)$. The rearrangement of each map at $t=500 \tau$ is shown in the bottom panel.

Figure 6.4 $\mathrm{B}$ plots the patterns of OPMs in one simulation with $\sigma=0.667 \sigma^{*}$. The maps evolved to a pattern of either stripes or pinwheel crystals. The behavior was qualitatively similar to $2 \mathrm{D}+\mathrm{nD}$ Gaussian feature space models with $n \geq 4$.

As a conclusion, neither in spherical feature space models or in toroidal feature space models any qualitative difference have been found in our simulations. 


\section{Chapter 7}

\section{Discussion and Conclusion of Part I}

Our study has revealed a multitude of dynamical phenomena and parameter dependencies in models of visual cortex development. These phenomena were previously overlooked although the models of feature mapping have been studied for more than 20 years (Kohonen, 1982, 1993; Obermaver et al., 1990; Ritter, 1990). In our work these phenomena became apparent by using a newly introduced quantitatively controlled method for simulating stimulus driven models of cortical development. Here we developed and presented this method that enables a partially analytical treatment and relatively convenient long-term simulations of the competitive Hebbian learning models. Nevertheless this method can and should be used generally.

\section{Methodology of modeling the developmental dy- namics}

In the study of a canonical developmental model for visual map formation presented above, we established a quantitatively controlled method for analysis and simulation of spatiotemporal dynamics, using key concepts of nonlinear dynamical systems theory. Our recipe for the analysis and simulation of a cortical developmental model can be summarized in the following steps:

1. Analyze the critical behavior at phase transitions to reveal the bifurcation threshold and the basic temporal and spatial scales of spontaneously symmetry breaking.

2. Systematically explore the dependence of pattern layouts on the system size so that system sizes can be chosen that are representative of large aspect ratio and weak confinement.

3. Perform numerical simulations in different dynamical regimes measuring 
simulation time in units of the intrinsic timescale such that long term stability can be accessed.

Each of the steps is reviewed below from our study of map formation and pinwheel dynamics in the Kohonen model. The mesoscopic level of description in the Kohonen model simplifies our problem, however, the type of phenomena we found are expected to be present fairly generally. Our method can be applied irrespective of model complexity and analytical tractability, especially with increased computing power and when dealing with a large complex spatio-temporal dynamics.

\section{Critical behavior at the phase transition}

In a dynamical system pattern formation was first analyzed close to the pattern formation threshold. Linear stability analysis of the Kohonen model (Chapter 2) revealed the pattern formation as a phase transition at a critical value of the coactivated cortical size $\sigma=\sigma^{*}$. The growth rate of the emerging patten (Eq. 2.20) defined an intrinsic time scale of the dynamics.

We worked out a methodology in order to quantitatively control simulations of models of different size, feature spaces of different dimensions, and different dynamical regimes. In order to approximate the deterministic dynamics from stochastic updating in the model, we chose the learning rate factor $\epsilon$ and the number of stimuli $N_{\text {stim }}$ such that a constant low noise level was maintained. We found that $\epsilon$ and $N_{\text {stim }}$ per se did not tell whether a simulation is to be considered to be long or short, noisy or deterministic. It turned out that these parameters had to be chosen according to the size of the system, the dimensionality of the feature spaces and the intrinsic timescale of the dynamics.

Often enough in a complicated dynamical system the critical threshold of pattern formation and the linear growth rate may not be derived analytically. In this case one can still apply this procedure by varying the control parameter in simulations and obtaining the threshold of phase transition and the time scale of intrinsic dynamics numerically as a basis for parameter choice and appropriate simulation time.

\section{Effects due to different system sizes}

Our simulations revealed that qualitatively different functional maps emerge in systems of different sizes. For small systems as $L=1 \Lambda_{\max }$, the layout of OPMs is quite regular with one simple stable pinwheel configuration. In all the other cases for $L \geq 2 \Lambda_{\max }$, initially irregular and pinwheel-rich spatial patterns only occur transiently. Either pinwheel annihilation or pinwheel crystallization occurred at the later stage. These observations show that the cortical network as a whole 
behaves in a way that is strongly influenced by factors beyond the properties of individual neurons.

To estimate the realistic systems size in the process of the cortical map development, we take an example of the striate cortex of Macaque Monkey. The size of system is measured by the aspect ratio $\Gamma$ such that

$$
\Gamma^{2}=\Pi / \Lambda^{2}
$$

where $\Pi$ measures the area of the monkey striate cortex, which is about $1000 \mathrm{~mm}^{2}$, and $\Lambda$ is the typical spacing of OPM recorded in area V1 of monkey, around $0.68 \mathrm{~mm}$ (Obermaver \& Blasdel, 1997). The calculated value of $\Gamma$ is about 50 .

In general it is computationally very demanding to solve the dynamics of such a large system with acceptable spatial resolution. Hence it was our strategy to first characterize behaviors of different-size systems and then work with a large enough system size so that it was sufficient to reveal the key features representing systems of realistic size.

In simulations we found strong confinement of map dynamics in a small system of $\Gamma=1$. Models of one hypercolumn have been studied before for the cortical network dynamics (see e.g. Hansel \& Sompolinsky (1996)). However, from our result such small systems appear not large enough to model the dynamics of map layout. Qualitatively different behaviors of pattern rearrangement were observed for $\Gamma \geq 2$. Our simulations revealed no qualitatively different behaviors in systems of $\Gamma=4$ and $\Gamma=5$, with similar kinetics of pinwheel annihilation in the low dimensional feature space models (Figure $4.8 \mathrm{E}, \mathrm{F}$ ). These results show that a system size of $\Gamma=4$ or $\Gamma=5$ is sufficient to reveal the phenomena and dynamical instability and rearrangement in the Kohonen model, which would be computationally very demanding to be resolved in a system of realistically large size.

\section{Qualitatively different regimes of the map dynamics}

Using this method, we performed a systematic study of the map dynamics in the Kohonen model. We found the model behaviors depended on the feature space dimensionality and the distance of the system from threshold.

In simulations with feature space dimension $n \leq 3$, the number of pinwheels was dramatically reduced by a dynamical process called pinwheel annihilation. Domain coarsening of spatial patterns was found to be more pronounced in systems far from threshold. The scaled pinwheel density measuring the abundance of pinwheels per hypercolumn decayed below 2 in all tested parameter regimes.

Dimension suppression was observed in systems close to threshold. In this regime only two feature dimensions were represented in the model cortex. The

model always behaved as low dimensional mapping exhibiting stripe-like patterns resulting from pinwheel annihilation. 
In feature space models of dimensionality $n \geq 4$, pinwheel crystallization was observed such that an initially pinwheel-rich aperiodic pattern was replaced by either stripes or crystals in different combinations of feature dimensions.

\section{Pinwheel crystals in the developmental model}

In simulations of systems far from threshold mapping a high dimensional feature space, we generally observed formation of regular 'checkerboard' patterns of pinwheel crystals. Pinwheel crystals have been previously reported in abstract models of OPMs aiming to minimize the wiring length (Koulakov \& Chklovskii, 2001) or to fulfill specific symmetry assumptions (Lee et al., 2003). Since such repetitive patterns have never been observed before in biologically plausible developmental models based on synaptic plasticity, many researchers in the field suspected that the regularity of pinwheel crystals is due to an oversimplification of these abstract 'physical' models. Our finding of pinwheel crystals in the Kohonen model solves this discrepancy and reveals that pinwheel crystallization represents a general property of stimulus driven models of Hebbian plasticity.

\section{The significance of pinwheel annihilation and pin- wheel crystallization}

Pinwheel annihilation (PWA) and pinwheel crystallization (PWC) appear to be dynamical phenomena robustly predicted by competitive Hebbian models of map development. So far neither of these has been observed in experiments. What are the implications of our finding for experimental studies of visual cortical dynamics? In principle there are three alternative interpretations of our results.

First, it is important to note that PWA and PWC are dynamical processes that take long time in map development. If they occur in the brain, it will require tracing maps in individual animals for weeks and months or potentially longer to demonstrate them in experiments. So far no such studies have been published. It is also interesting to note that most of the reported maps were recorded in juvenile animals due to technical reasons. It is conceivable that maps only exhibit signatures of PWC in the fully mature brain. From this perspective, our results raise the urgency of chronic long-term imaging in developing and mature animals.

Second, there might be unknown biological mechanisms that stop the rearrangement of OPMs early in development potentially before the end of the critical period. It is however more plausible to imagine molecular cues that set up an initially crude pattern of cortical maps, whereas the further maturation and refinement of the maps involves an activity dependent process driven by sensory experience. The function of this stimuli-driven process of pattern refinement becomes ambiguous if an internal signal would be needed to stop this process. 
Finally, some essential ingredient might be missing in the considered model of feature mapping. A phenomenological model based on an abstract order parameter field (Wolf, 2005) has recently been used to examine conditions for the stabilization of aperiodic patterns. This model converges towards multi-stable attractors when pinwheels are stabilized by long-range interactions. More biologically plausible implementations of this type of model are required to further explore this possibility.

\section{Dimension suppression versus dimension reduction}

One of the most surprising results in our study was the dimension suppression observed in systems close to the critical point, where the model cortex only encoded two features of the visual stimuli, no matter how many dimensions were present in the feature space. A new bifurcation point $\sigma^{* *}$ was found in our simulations of high dimensional feature space models such that additional feature dimensions were suppressed until the size of co-activated cortical domains was below the secondary bifurcation $\sigma<\sigma^{* *}$.

Thus how far the dynamics is away from the critical point determines how many independent maps are encoded in the cortex. If the system lies in the dimension suppression regime $\left(\sigma^{* *}<\sigma<\sigma^{*}\right)$, only two feature dimensions are active, which contradicts a core assumption of the dimension reduction framework. The model cortex does not equally represent all conceivable combinations of stimulus features, regardless of the fully symmetric stimulus distribution in the feature spaces that has been used.

From this perspective it is intriguing that a recent experimental study has questioned the conventional view that visual cortical activity pattern consists of a superposition of different feature maps in one cortical area (Basole et al., 2003). The authors argued that the observed intersection of different maps was biased by the use of grating stimuli, in which the examined range of motion and spatial frequency is limited to those varying along an axis orthogonal to the grating's orientation. Applying texture stimuli of different bar lengths, motion directions and speeds, they found that stimuli of the same orientation can elicit responses of different neural populations and the same neural populations can be activated by multiple combination of orientation, length, motion direction and speed. Rather than overlapping multiple maps of different features, an alternative framework of stimulus energy model was proposed to describe the cortical activity as a single map of spatiotemporal energy (Basole et al., 2003; Mante \& Carandini, 2005).

Our results show that dynamical models of cortical development naturally exhibit a regime where such a functional architecture with a reduced number of stimulus feature dimensions is predicted to form. 


\section{Conclusion}

The Kohonen model has been used in many studies to explain the formation of topographic maps such as the tonotopic map, the retinotopic map and the functional maps like ODCs and OPMs (Erwin et al., 1992a b; Graepel \& Obermaver, 1999; Kohonen, 1982; Mitchison \& Swindale, 1999; Swindale, 2000, 2004). It is considered representative for a large class of competitive Hebbian models for the development of orientation columns in the visual cortex.

In this thesis, we characterized the dynamics of OPMs using a newly developed quantitative controlled numerical methodology. We studied $2 \mathrm{D}+2 \mathrm{D}$ model behaviors varying system sizes and quantified pinwheel dynamics and stability. If only orientation preference and retinotopy were mapped on the cortex, the initially emerging OPMs are generally unstable due to a process known as pinwheel annihilation.

Our results correct previous simulation studies that followed an ad hoc methodology. In one set of such simulations (e.q.. Swindale (2004): Goodhill \& Cimponeriu (2000)), the size $\sigma$ of co-activated cortical domains was reduced as the map developed. Since more co-activated domains were recruited, it would require very long simulation time (Eq.(3.5) ) to track the dynamics. As $\sigma$ tends to zero, this approach leads not to a final convergence of the model but an artificial termination of map dynamics. Other studies restricted the dynamics of the system by using a small set of fixed stimuli repeated over and over to train the network (Durbin \& Mitchison, 1990; Goodhill \& Cimponeriu, 2000). We demonstrated here that the resulting 'frozen' patterns of OPMs become unstable when more random stimuli are presented.

A previous study proposed that interactions with ODCs or other maps may stabilize pinwheels (Wolf \& Geisel, 1998). Our study comprehensively investigated this hypothesis by including more feature dimensions in the model and systematically examining their impact. Close to threshold, dimension suppression was observed in simulations so that the system behaved similarly to a system with a 2D feature space exhibiting unstable pinwheel dynamics. Pinwheel crystallization was observed in systems far from threshold. Similar results were observed for Gaussian distributions, for spherical uniform distributions, and for products of circular uniform distributions.

We conclude that nonlinear dynamical systems theory provides a quantitative method to study the developmental behaviors of cortical models. Using this newly developed method we systematically simulated the dynamical processes of competitive Hebbian learning in map formation. The processes were characterized by the kinetics of pinwheel annihilation and the phenomena of dimension suppression and pinwheel crystallization in different dynamical regimes. These new phenomena appeared to be robust in the developmental models but have not yet been discovered in experimental recordings. To test the model prediction of pinwheel crystallization will require chronic long-term imaging in developing and 
adult animals. Alternatively, if pinwheels show no indication of crystallization in animals, further theoretical studies are required to uncover the important missing feature in competitive Hebbian models of cortical development. 


\section{Bibliography}

Basole, A., White, L. E., \& Fitzpatrick, D. (2003). Mapping multiple features in the population response of visual cortex. Nature, 423, 986-990.

Blasdel, G. G. \& Salama, G. (1986). Voltage-sensitive dyes reveal a modular organization in monkey striate cortex. Nature, 321, 579-585.

Bonhoeffer, T. \& Grinvald, A. (1991). Iso-orientation domains in cat visual cortex are arranged in pinwheel-like patterns. Nature, 353, 429-431.

Bosking, W. H., Zhang, Y., Schofield, B., \& Fitzpatrick, D. (1997). Orientation selectivity and the arrangement of horizontal connections in tree shrew striate cortex. J Neurosci, 17, 2112-2127.

Carreira-Perpiñán, M. A., Lister, R. J., \& Goodhill, G. J. (2005). A computational model for the development of multiple maps in primary visual cortex. Cereb Cortex, 15, 1222-1233.

Chapman, B., Stryker, M. P., \& Bonhoeffer, T. (1996). Development of orientation preference maps in ferret primary visual cortex. J Neurosci, 16, 6443-6453.

Cho, M. W. \& Kim, S. (2004). Understanding visual map formation through vortex dynamics of spin hamiltonian models. Phys. Rev. L, 92, 018101.

Crair, M. C., Gillespie, D. C., \& Stryker, M. P. (1998). The role of visual experience in the development of columns in cat visual cortex. Science, 279, $566-570$.

Crair, M. C., Ruthazer, E. S., Gillespie, D. C., \& Stryker, M. P. (1997a). Ocular dominance peaks at pinwheel center singularities of the orientation map in cat visual cortex. J Neurophysiol, 77, 3381-3385.

Crair, M. C., Ruthazer, E. S., Gillespie, D. C., \& Stryker, M. P. (1997b). Relationship between the ocular dominance and orientation maps in visual cortex of monocularly deprived cats. Neuron, 19, 307-318.

Cross, M. C. \& Hohenberg, P. (1993). Pattern formation out of equilibrium. Reviews of Modern Physics, 65, 851-1112. 
Durbin, R. \& Mitchison, G. (1990). A dimension reduction framework for understanding cortical maps. Nature, 343, 644-647.

Erwin, E., Obermayer, K., \& Schulten, K. (1992a). Self-organizing maps: ordering, convergence properties and energy functions. Biol Cybern, 67, 47-55.

Erwin, E., Obermayer, K., \& Schulten, K. (1992b). Self-organizing maps: stationary states, metastability and convergence rate. Biol Cybern, 67, 35-45.

Farley, B. J., Yu, H., Jin, D. Z., \& Sur, M. (2007). Alteration of visual input results in a coordinated reorganization of multiple visual cortex maps. J Neurosci, 27, 10299-10310.

Ferster, D. \& Miller, K. D. (2000). Neural mechanisms of orientation selectivity in the visual cortex. Annu Rev Neurosci, 23, 441-471.

Frostig, R. D., Lieke, E. E., Ts'o, D. Y., \& Grinvald, A. (1990). Cortical functional architecture and local coupling between neuronal activity and the microcirculation revealed by in vivo high-resolution optical imaging of intrinsic signals. Proc Natl Acad Sci U S A, 87, 6082-6086.

Godde, B., Leonhardt, R., Cords, S. M., \& Dinse, H. R. (2002). Plasticity of orientation preference maps in the visual cortex of adult cats. Proc Natl Acad Sci U S A, 99, 6352-6357.

Goodhill, G. J. \& Cimponeriu, A. (2000). Analysis of the elastic net model applied to the formation of ocular dominance and orientation columns. Network, 11, $153-168$.

Graepel, T. \& Obermayer, K. (1999). A stochastic self-organizing map for proximity data. Neural Comput, 11, 139-155.

Hansel, D. \& Sompolinsky, H. (1996). Chaos and synchrony in a model of a hypercolumn in visual cortex. J Comput Neurosci, 3, 7-34.

Hebb, D. (1949). The Organization of Behavior. (New York: Wiley).

Hubel, D. (1995). Eye, brain and vision. New York, Sci.Am.Library.

Hubel, D. H. \& Wiesel, T. N. (1962). Receptive fields, binocular interaction and functional architecture in the cat's visual cortex. J Physiol, 160, 106-154.

Hübener, M., Shoham, D., Grinvald, A., \& Bonhoeffer, T. (1997). Spatial relationships among three columnar systems in cat area 17. J Neurosci, 17, 9270-9284.

Kaschube, M. (2005). Pattern selection in the visual cortex. PhD thesis. 
Kaschube, M., Schnabel, M., Löwel, S., Coppola, D. M., White, L. E., \& Wolf, F. (2006). Universal pinwheel statistics in the visual cortex. Society for Neuroscience Abstract, Program No.545.9.

Kaschube, M., Wolf, F., Geisel, T., \& Löwel, S. (2000). Quantifying the variability of patterns of orientation domains in the visual cortex of cats. Neurocomputing, $32-33,415-423$.

Katz, L. C. \& Crowley, J. C. (2002). Development of cortical circuits: lessons from ocular dominance columns. Nat Rev Neurosci, 3, 34-42.

Kohonen, T. (1982). Self-organized formation of topologically correct feature maps. Biol. Cybern., 43, 59-69.

Kohonen, T. (1993). Physiological interpretation of the self-organizing map algorithm. Neural Netw., 6, 895-905.

Koulakov, A. A. \& Chklovskii, D. B. (2001). Orientation preference patterns in mammalian visual cortex: a wire length minimization approach. Neuron, 29, $519-527$.

Lee, H. Y., Yahyanejad, M., \& Kardar, M. (2003). Symmetry considerations and development of pinwheels in visual maps. Proc Natl Acad Sci U S A, 100, 16036-16040.

Löwel, S., Freeman, B., \& Singer, W. (1987). Topographic organization of the orientation column system in large flat-mounts of the cat visual cortex: a 2deoxyglucose study. J Comp Neurol, 255, 401-415.

Löwel, S., Schmidt, K. E., Kim, D. S., Wolf, F., Hoffsümmer, F., Singer, W., \& Bonhoeffer, T. (1998). The layout of orientation and ocular dominance domains in area 17 of strabismic cats. Eur J Neurosci, 10, 2629-2643.

Löwel, S. \& Wolf, F. (1999). Pattern formation in the developing visual cortex. Lecture Notes in Physics, 532, 1-29.

Manneville, P. (1990). Dissipative Structure and Weak Turbulence (Perspectives in Physics). (San Diego: Academic Press).

Mante, V. \& Carandini, M. (2005). Mapping of stimulus energy in primary visual cortex. J Neurophysiol, 94, 788-798.

Miller, K. D. (1992). Development of orientation columns via competition between on- and off-center inputs. Neuroreport, 3, 73-76. 
Miller, K. D. (1994). A model for the development of simple cell receptive fields and the ordered arrangement of orientation columns through activitydependent competition between on- and off-center inputs. J Neurosci, 14, 409-441.

Miller, K. D., Keller, J. B., \& Stryker, M. P. (1989). Ocular dominance column development: analysis and simulation. Science, 245, 605-615.

Mitchison, G. J. \& Swindale, N. V. (1999). Can hebbian volume learning explain discontinuities in cortical maps? Neural Comput, 11, 1519-1526.

Mooser, F., Bosking, W. H., \& Fitzpatrick, D. (2004). A morphological basis for orientation tuning in primary visual cortex. Nat Neurosci, 7, 872-879.

Müller, T., Stetter, M., Hübener, M., Sengpiel, F., Bonhoeffer, T., Gödecke, I., Chapman, B., Löwel, S., \& Obermayer, K. (2000). An analysis of orientation and ocular dominance patterns in the visual cortex of cats and ferrets. Neural Comput, 12, 2573-2595.

Obermayer, K. \& Blasdel, G. G. (1997). Singularities in primate orientation maps. Neural Comput, 9, 555-575.

Obermayer, K., Blasdel, G. G., \& Schulten, K. (1992). Statistical-mechanical analysis of self-organization and pattern formation during the development of visual maps. Physical Review. A, 45, 7568-7589.

Obermayer, K., Ritter, H., \& Schulten, K. (1990). A principle for the formation of the spatial structure of cortical feature maps. Proc Natl Acad Sci U S A, 87, 8345-8349.

Ohki, K., Chung, S., Ch'ng, Y. H., Kara, P., \& Reid, R. C. (2005). Functional imaging with cellular resolution reveals precise micro-architecture in visual cortex. Nature, 433, 597-603.

Ohki, K., Chung, S., Kara, P., Huebener, M., Bonhoeffer, T., \& Reid, R. C. (2006). Highly ordered arrangement of single neurons in orientation pinwheels. Nature, 442, 925-928.

Ritter, H. (1990). Self-organizing maps for internal representations. Psychol Res, 52, 128-136.

Ritter, H. \& Schulten, K. (1988). Convergence properties of kohonen's topology conserving maps: fluctuations, stability, and dimension selection. Biol. Cybern., 60, 59-71.

Sengpiel, F., Stawinski, P., \& Bonhoeffer, T. (1999). Influence of experience on orientation maps in cat visual cortex. Nat Neurosci, 2, 727-732. 
Sharma, J., Angelucci, A., \& Sur, M. (2000). Induction of visual orientation modules in auditory cortex. Nature, 404, 841-847.

Sur, M. \& Leamey, C. A. (2001). Development and plasticity of cortical areas and networks. Nat Rev Neurosci, 2, 251-262.

Swindale, N. V. (2000). How many maps are there in visual cortex? Cereb Cortex, 10, 633-643.

Swindale, N. V. (2004). How different feature spaces may be represented in cortical maps. Network, 15, 217-242.

Weliky, M., Bosking, W. H., \& Fitzpatrick, D. (1996). A systematic map of direction preference in primary visual cortex. Nature, 379, 725-728.

Weliky, M. \& Katz, L. C. (1999). Correlational structure of spontaneous neuronal activity in the developing lateral geniculate nucleus in vivo. Science, 285, 599604 .

White, L. E. \& Fitzpatrick, D. (2007). Vision and cortical map development. Neuron, 56, 327-338.

Wolf, F. (2005). Symmetry, multistability, and long-range interactions in brain development. Phys. Rev. Lett., 95.

Wolf, F. \& Geisel, T. (1998). Spontaneous pinwheel annihilation during visual development. Nature, 395, 73-78.

Wolf, F., Pawelzik, K., Scherf, O., Geisel, T., \& Löwel, S. (2000). How can squint change the spacing of ocular dominance columns? J Physiol Paris, 94, 525-537.

Yu, H., Farley, B. J., Jin, D. Z., \& Sur, M. (2005). The coordinated mapping of visual space and response features in visual cortex. Neuron, 47, 267-280. 


\section{Part II}

\section{Action Potential Dynamics and Response Function of Cortical Neuron Models}





\section{Chapter 8}

\section{Introduction}

\subsection{Action Potential Dynamics in Phase Plots}

To study the function of central nervous system, it is vital to concentrate not only on its functional architecture of cortical columns but also on the properties of its constituents, that allow for a plethora of cognitive functions. A key problem in modeling the behavior of individual neurons is to identify their input-output function translating synaptic inputs into spike trains by the action potential (AP) generator under in vivo-like conditions of operation. Biophysically detailed models have a large number of parameters that are often manually tuned to fit some set of experimental data, which might be neither unique nor effective in predicting a neuron's behaviors. One simplified but remarkably informative approach is provided by phase plots of action potentials.

As shown in Figure 8.1 a phase plot is derived from the voltage-time record of spikes such that the time derivative of the voltage $(\mathrm{d} V / \mathrm{d} t)$ is displayed as a function of the voltage. An action potential is represented by a loop in phase plots. The threshold potential for the AP onset can be immediately read out from the phase plot as the voltage with a sudden increase of $\mathrm{d} V / \mathrm{d} t$.

In vivo cortical neurons are bombarded by ten thousands of synaptic inputs every second but fire APs only very infrequently (Greenberg et al., 2008). To examine the rules by which cortical neurons turn fluctuating inputs into a precise pattern of outgoing spikes, various groups have used phase plots obtained from intracellular voltage recordings (Naundorf et al., 2005b; Volgushev et al., 2008; Jolivet et al., 2008). These studies indicated that there is no simple procedure to predict the timing of the outgoing APs from the subthreshold responses preceding the spikes. For instance, Naundorf et al. (2006) showed that there apparently is no unique threshold potential for APs in visual cortical neurons observed in vivo. Badel et al. (2008) used phase plots to fit simple neuron models to similar intracellular recordings. They showed that models matching the key features of phase plots are currently the best models for predicting the precise spike sequences 

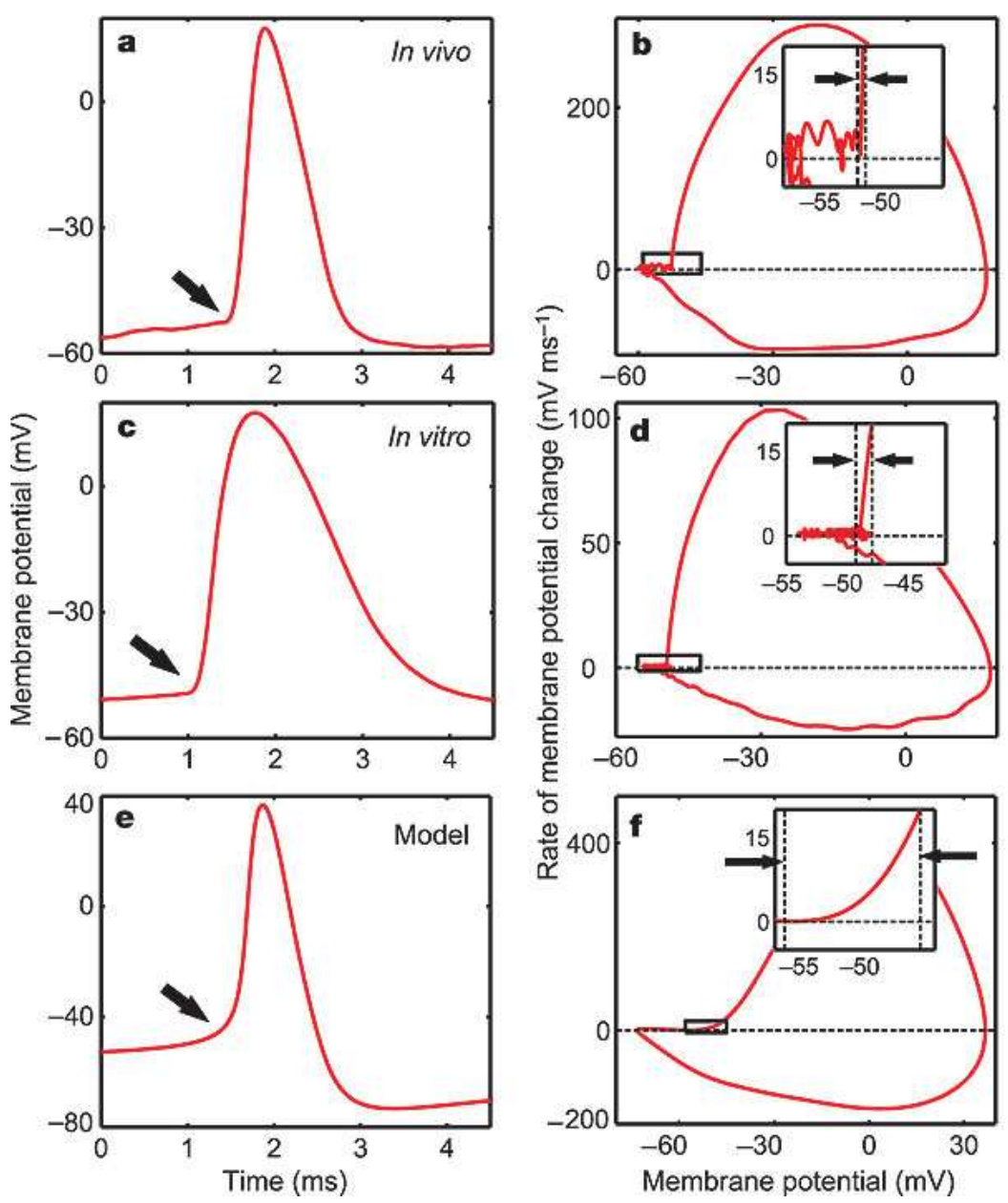

Figure 8.1: Dynamics of action potential initiation in neocortical neurons and in a Hodgkin-Huxley type model. (a), Action potential measured in a cat's visual cortical neuron in vivo. The arrow shows the characteristic kink at action potential onset. (b), Phase plots $(\mathrm{dV} / \mathrm{dt}$ versus $\mathrm{V})$ of the action potential from (a). Inset shows the initial phase of the action potential. (c, d), Action potential from a cat's visual cortical slice in vitro at $20^{\circ} \mathrm{C}$. (e, f). Action potential from a Hodgkin-Huxley-type model. Figure taken from Naundorf et al. $(2006)$ 
of cortical neurons for in vivo-like inputs.

\subsection{Population Coding}

Cognitive functions such as visual attention require fast, reliable processing of stimuli (Thorpe et al., 1996; Hopfield, 1995; Steinmetz et al., 2000), e.g. . to recognize a natural image the human visual svstem reauires less than $150 \mathrm{~ms}$ (Thorpe et al., 1996). On the other hand, individual neurons in the visual cortex are silent most of the time and fire only a few APs per second under many conditions (Greenberg et al., 2008).

In order to follow fast changing stimuli containing temporal frequencies much higher than the firing rate of a single neuron, it requires coordinated activity of a large population of neurons. The population activity revealed by the local field potentials recorded in the cerebral cortex in fact exhibits much faster rhythms than the firing frequency of individual neurons (Buzsáki \& Draguhn, 2004). Whereas a single neuron may not fire in each cycle, the instantaneous firing rate averaged over an neuronal ensemble sustains a pattern of fast oscillation. To understand such a collective behavior of cortical activity, it is crucial to study the spike generation mechanism of individual neurons in the presence of time-varying inputs and background synaptic noise. Only if a neuron can time its spikes with sufficient temporal precision can it participate in a fast population rate coding.

Recent experimental evidence has revealed that cortical neurons in vitro reliably transmit a noisv sinusoidal input up to a frequency of $200 \mathrm{~Hz}$ (Köndgen et al., 2008). In contrast, responses were found to be much slower in conductance based (CB) models compared to the cortical neurons' response (FourcaudTrocmé et al., 2003; Naundorf et al., 2005b). Such a discrepancy calls for deep examination of the spike generation mechanism in biophysical neuron models.

\subsection{Fast Onset Dynamics of AP Initiation}

What is the intrinsic property of a single neuron that endows a population of cortical neurons with the ability to follow high frequency input? Previous theoretical studies have suggested that the properties of single neuron AP generation determine the response speed of a neuron ensemble to fast changing stimuli, with a fast onset of the spikes leading to a more reliable response to high frequency inputs (Fourcaud-Trocmé et al., 2003; Naundorf et al., 2005a, 2006).

Such a rapid rate of rise at the onset of somatic APs (Figure 8.1) has been indeed observed in in vitro and in vivo recordings of cortical neurons (Naundorf et al., 2006; Volgushev et al., 2008). The dynamics of AP initiation is characterized by an abrupt onset in phase plots, which significantly deviated from the 
behavior of canonical Hodgkin-Huxley-type models.

The biological processes leading to a fast onset of action potentials measured at the soma (an experimentally more accessible region compared to axon) have been a topic of heated debate in recent theoretical and experimental studv (Naundorf et al.. 2006: Volgushev et al.|.2008: Kole et al.. 2008: Yu et al..|2008). Naundorf et al. (2006) proposed one possible mechanism to induce a rapid rate of rise at spike onset as cooperative gating of sodium channels, which are presumed to act independently in Hodgkin-Huxley-type models. The mechanism of channel cooperativity is first implemented in biophysical models for AP generation in our study. We further explore the firing patterns of these models in response to time-varying noisy inputs.

\subsection{AP Initiation and Back-Propagation in Multi- Compartment Models}

Neurophysiologists however have raised strong criticisms against the use of phase plots to study action potential dynamics (McCormick et al., 2007; Bean, 2007). This criticism concerns the assumption that $\mathrm{d} V / \mathrm{d} t$ derived from somatic recording can be effectively used as a proxy of membrane current in such studies. This simplifying assumption may be violated in real neurons, which are spatially extended systems that are more accurately described using multi-compartment models coupled by lateral currents. If these lateral currents were overwhelmingly strong, examination of phase plots from the somatic recording could be misleading.

A model of multi-compartment Hodgkin-Huxley-type neurons has been recently published to support the 'lateral current' hypothesis (Yu et al., 2008). The authors claimed that a spike initiated at the axon initial segment (AIS) rises as smoothly as described by the Hodgkin-Huxley-model; due to spatial inhomogeneity and the large lateral current supplied by the axonal spike, the AP back-propagating into soma resulted in a rapid rising phase.

Whether or not this is the case however is a quantitative rather than a qual-

itative question. It critically depends on the exact amount and the timing of lateral currents in real neurons, a question that requires exact knowledge of the dependence of lateral currents on a neurons's morphology and physiological parameters. To address this problem systematically, we performed extensive simulations of multi-compartment models and critically examine to what extent key properties of phase plots are affected by lateral currents.

\subsection{An Overview of my Work}

The main purpose of this study is to investigate the phase plot dynamics of action potentials in different conductance-based models, and moreover, to explore the 
spike generation mechanism of cortical neurons with fast response properties. The second part of this thesis is organized as follows:

We first describe the Wang-Buzsaki model and other Hodgkin-Huxley-type models for cortical AP generation in Chapter 9. We further introduce the OrnsteinUhlenbeck stochastic process used to mimic cortical synaptic noise. The neuron's response function to noisy inputs is characterized by both subthreshold MP fluctuation and superthreshold firing patterns of a population.

In Chapter 10, we construct a cooperative Wang-Buzsaki ('cWB') model that implements the mechanism of channel cooperativity. We characterize the AP dynamics and coding properties in this model with variable coupling strength of cooperative channels.

The phase plot dynamics of the somatic APs are first studied in a multicompartment neuron model with back propagation of antidromic APs (Chapter 11). Using this simplified model, we ask how the AP waveforms, in particular the onset dynamics in the phase plots, are influenced by the neuron morphology such as soma geometry and propagation distance from the AP initiation site to the soma.

In Chapter 12, we further address the questions of AP initiation sites and somatic AP waveforms using more realistic neuron models with inhomogeneous physiological properties across the soma, axon and dendrite. Then we explore the frequency coding properties of different multi-compartment models characterized by different onset dynamics of somatic APs in the phase plots.

The main results of our study are discussed in Chapter 13. 


\section{Chapter 9}

\section{Basics of Dynamic Response in Conductance Based Models}

This chapter first introduces the Wang-Buzsaki model for AP generation in cortical neurons. The background synaptic noise is mimicked by injecting the model with a stochastic Ornstein-Uhlenbeck current. We characterize the properties of neuronal response to noisy inputs by both the subthreshold MP fluctuation and the superthreshold frequency modulation of spikes.

\subsection{Computational Models}

\subsubsection{Wang-Buzsaki Models of AP Generation}

We use the Wang-Buzsaki model as a representative of conductance-based (CB) models in most of our study. This model, first introduced by Wang \& Buzsáki (1996), follows the original formalism of Hodgkin-Huxlev equations (Hodgkin \& Huxley, 1952), where the AP generator consists of voltage-dependent $\mathrm{Na}^{+}$and $\mathrm{K}^{+}$currents. The Wang-Buzsaki model makes a simplification that $\mathrm{Na}^{+}$channels response to a voltage step with instantaneous activation. This model exhibiting type I excitability has been explored in a line of theoretical studies on the dynamical firing properties of cortical neurons (Wang \& Buzsáki. 1996: Hansel \& Mato, 2003; Fourcaud-Trocmé \& Brunel, 2005; Naundorf et al., 2005b).

The dynamics of the membrane potential $V(t)$ is governed by the current balance equation:

$$
C_{\mathrm{M}} \frac{\mathrm{d} V}{\mathrm{~d} t}=-I_{\mathrm{Na}}-I_{\mathrm{K}}-I_{\mathrm{L}}+I_{\mathrm{app}},
$$

where $C_{\mathrm{M}}$ is the membrane capacitance, $I_{\mathrm{Na}}$ and $I_{\mathrm{K}}$ are ionic currents from voltage-gated sodium and potassium channels, $I_{\mathrm{L}}$ are ionic currents from an unspecific leak conductance, and $I_{\text {app }}$ are the applied currents from the electrode.

The ionic currents are calculated from Ohm's law, where the conductance is a product of the maximum conductance and the opening probability of individual 
channels:

$$
\begin{aligned}
\mathrm{I}_{\mathrm{Na}} & =\bar{g}_{\mathrm{Na}} m^{3} h\left(V-E_{\mathrm{Na}}\right), \\
\mathrm{I}_{\mathrm{k}} & =\bar{g}_{\mathrm{K}} n^{4}\left(V-E_{\mathrm{K}}\right), \\
\mathrm{I}_{\mathrm{L}} & =g_{\mathrm{L}}\left(V-E_{\mathrm{L}}\right),
\end{aligned}
$$

and $E_{\mathrm{Na}}, E_{\mathrm{K}}$ and $E_{\mathrm{L}}$ are reversal potential of the corresponding ion channels. The fast activation of $\mathrm{Na}^{+}$channels is assumed to be instantaneous such that $m$ can be substituted by its steady-state function $m_{\infty}$. The dynamical equation for the gating variables $h$ and $n$ is represented by

$$
\tau_{x} \frac{\mathrm{d} x}{\mathrm{~d} t}=x_{\infty}-x
$$

where $x=h, n$. The steady state value $x_{\infty}$ and time constant $\tau_{x}$ are given by

$$
\begin{gathered}
x_{\infty}=\frac{\alpha}{\alpha+\beta}, \\
\tau_{x}=\frac{1}{\alpha+\beta},
\end{gathered}
$$

where $\alpha$ and $\beta$ represent forward and backward reaction rates, respectively. They are assumed to be exclusively dependent on the local membrane potential, which are explicitly specified as functions of $V$ (see table 9.1).

Another type of widely used models for AP generation in cortical neurons is the Mainen-Sejnowski model (Mainen et al., 1995; Mainen \& Sejnowski, 1996). The model equations are similar as those in Wang-Buzsaki model except that the $\mathrm{K}^{+}$channel opening has only one instead of four subunit gates. In addition, the gating variable $m$ for $\mathrm{Na}^{+}$channel activation follows $\mathrm{Eq} 9.3$ with a finite activation time constant.

All the voltage-dependent functions describing channel kinetics and physiological parameters are summarized in Table 9.1 and Table 9.2 in the appendix to this chapter.

\subsubsection{Modeling Background Noise in the Cortex}

In the cerebral cortex neurons are embedded in a large network with numerous connections of excitatory and inhibitory synapses. The background noise from spontaneous cortical activity can be characterized by Gaussian statistics (Figure 9.1) and by an autocorrelation function exponentially decaying with time constant $\tau_{I}$. Such intense network activity in awake animals is supposed to cause a remarkable membrane potential fluctuation and to alter the neuronal response property as compared to the resting states induced bv TTX or anesthesia (Destexhe \& Paré, 1999; Steriade, 2001; Greenberg et al., 2008). 


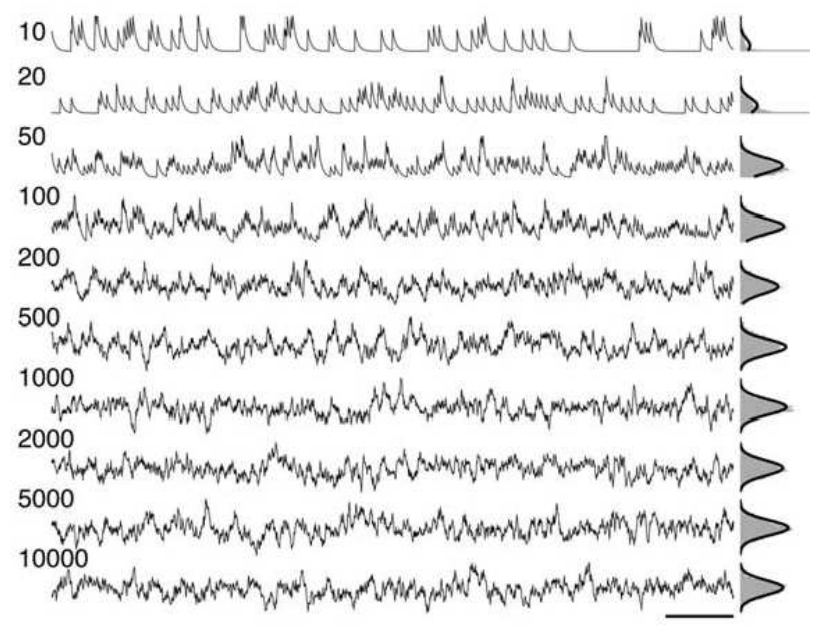

Figure 9.1: The total synaptic inputs from a presynaptic neuronal population of increasing size. The figure shows a model simulation of a generic neuron that receives independent inputs from the intra-cortical network through identical AMPA synapses $\left(\tau_{I}=10 \mathrm{~ms}\right)$. Numbers of synaptic connections are indicated on the left column, while each of the presynaptic neurons spontaneously fires at a rate as low as $5 \mathrm{~Hz}$. The post-synaptic currents approach a Gaussian distribution by the central-limit theorem for more than 100 synapses. (Adapted from Giugliano et al. (2006)) 
Recently there have been several studies using computer-synthesized noisy current $I_{\text {syn }}(t)$ to mimic the in vivo synaptic input, which was then injected through current-clamp to the soma in slice preparation (Rauch et al., 2003: Silberberg et al., 2004; Köndgen et al., 2008). This procedure allows one to study the capability of cortical neurons to track time varying signals and the impact of the background noise on the neuronal response property.

Following a classical approach in the theory of stochastic system (Gardiner, 1994), the background noise $I_{\text {syn }}(t)$ is modeled as statistically stationary input generated from an Ornstein-Uhlenbeck process:

$$
\tau_{I} \dot{I}_{\mathrm{syn}}=-I_{\mathrm{syn}}+A^{\prime} \xi_{t}
$$

where $\xi_{t}$ is a random variable drawn at every time step from a Gaussian distribution with a mean of zero, called white noise. By intergrating the deterministic part of Eq. 9.5.

$$
I_{\text {syn }}(t+\Delta t)=I_{\text {syn }}(t) \exp \left(-\Delta t / \tau_{I}\right)+A \xi_{t}
$$

The variance of the variable $I_{\text {syn }}(t)$ is given by

$$
\begin{gathered}
\left\langle I_{\mathrm{syn}}^{2}\right\rangle=\left\langle\left[I_{\mathrm{syn}}(t) \exp \left(-\Delta t / \tau_{I}\right)+A \xi_{t}\right]^{2}\right\rangle=\sigma_{I}^{2}, \\
\left\langle I_{\mathrm{syn}}^{2}\right\rangle=\left\langle I_{\mathrm{syn}}^{2}\right\rangle \exp \left(-2 \Delta t / \tau_{I}\right)+2 A\left\langle I_{\mathrm{syn}} \cdot \xi_{t}\right\rangle \exp \left(-\Delta t / \tau_{I}\right)+A^{2}\left\langle\xi_{t}^{2}\right\rangle .
\end{gathered}
$$

Since $I_{\text {syn }}$ and $\xi_{t}$ are independent variables their correlation $\left\langle I_{\text {syn }} \cdot \xi_{t}\right\rangle=0$. The prefactor A can be adjusted by setting the variance of $I_{\mathrm{syn}}(t)$ as $\sigma_{I}$ :

$$
A=\sigma_{I} \sqrt{1-\exp \left(-2 \Delta t / \tau_{I}\right)}
$$

After applying the Taylor expansion and neglecting the higher order terms, $I_{\text {syn }}(t)$ defined in Eq. 9.5 can be obtained by time discrete iteration scheme:

$$
I_{\mathrm{syn}}(t+\Delta t)=I_{\mathrm{syn}}(t)\left(1-\Delta t / \tau_{I}\right)+\sigma_{I} \sqrt{1-\exp \left(-2 \Delta t / \tau_{I}\right)} \xi_{t} .
$$

\subsection{Subthreshold Response to Noisy Inputs}

\section{Direct Solutions of the Linear Dynamics}

We first study the subthreshold fluctuation of membrane potential, where voltage dependent $-\mathrm{Na}^{+}$and $-\mathrm{K}^{+}$channels are not yet active, hence the original HodgkinHuxley-type equations can be reduced to a linear model of a leak conductance $g_{L}$, an injected constant current $I_{0}$, and a synaptic current $I_{\text {syn }}(t)$ :

$$
c \frac{\mathrm{d} V}{\mathrm{~d} t}=-g_{L} V+I_{0}+\sigma_{I} I_{\mathrm{syn}}(t)
$$


where $c$ is the membrane capacitance.

The synaptic current is modeled as an Ornstein-Uhlenbeck process:

$$
\frac{\mathrm{d} I_{\text {syn }}}{\mathrm{d} t}=-\frac{I_{\text {syn }}}{\tau_{I}}+\xi(t)
$$

where $\tau_{I}$ is the decay time constant and $\xi(t)$ is a Gaussian random variable. Hence the temporal correlation of the synaptic current is described by

$$
C(\tau)=\exp \left(-\frac{|\tau|}{\tau_{I}}\right)
$$

The solution to Eq.(9.7) can be written as

$$
V(t)=V_{0} \exp \left(-\frac{t}{\tau_{M}}\right)+\frac{1}{c} \int_{0}^{t} \mathrm{~d} t^{\prime}\left(I_{0}+\sigma_{I} I_{\text {syn }}\left(t^{\prime}\right)\right) \exp \frac{t^{\prime}-t}{\tau_{M}} .
$$

The temporal average of $V(t)$ is given by

$$
\langle V(t)\rangle=\left(V_{0}-\frac{I_{0}}{g_{L}}\right) \exp \left(-\frac{t}{\tau_{M}}\right)+\frac{I_{0}}{g_{L}} .
$$

The initial state of $\mathrm{V}(\mathrm{t})$ is forgotten if $t \gg \tau_{M}$ so that the mean of $\mathrm{V}(\mathrm{t})$ is determined by the constant input $\langle V(t)\rangle=I_{0} / g_{L}$. The variance of MP fluctuation is given by $\sigma_{V}^{2}=\left\langle(V(t)-\langle V(t)\rangle)^{2}\right\rangle$, where

$$
\begin{aligned}
& V(t)-\langle V(t)\rangle=\frac{\sigma_{I}}{c} \int_{0}^{t} \mathrm{~d} t^{\prime} I_{\text {syn }}\left(t^{\prime}\right) \exp \left(\frac{t^{\prime}-t}{\tau_{M}}\right) . \\
& \sigma_{V}^{2}=\frac{\sigma_{I}^{2}}{c^{2}}\left\langle\int_{0}^{t} I_{\text {syn }}\left(t^{\prime}\right) \exp \left(\frac{t^{\prime}-t}{\tau_{M}}\right) \mathrm{d} t^{\prime} \int_{0}^{t} I_{\text {syn }}\left(t^{\prime \prime}\right) \exp \left(\frac{t^{\prime \prime}-t}{\tau_{M}}\right) \mathrm{d} t^{\prime \prime}\right\rangle, \\
&=\frac{\sigma_{I}^{2}}{c^{2}} \int_{0}^{t} \int_{0}^{t} \exp \left(\frac{t^{\prime}+t^{\prime \prime}-2 t}{\tau_{M}}\right)\left\langle I_{\text {syn }}\left(t^{\prime}\right) I_{\text {syn }}\left(t^{\prime \prime}\right)\right\rangle \mathrm{d} t^{\prime} \mathrm{d} t^{\prime \prime} \\
&= \frac{2 \sigma_{I}^{2}}{c^{2}} \int_{0}^{t} \mathrm{~d} t^{\prime} \int_{0}^{t^{\prime}} \exp \left(\frac{t^{\prime}+t^{\prime \prime}-2 t}{\tau_{M}}\right) \exp \left(\frac{-\left(t^{\prime}-t^{\prime \prime}\right)}{\tau_{I}}\right) \\
&= \frac{2 \sigma_{I}^{2}}{c^{2}} \int_{0}^{t} \mathrm{~d} t^{\prime} \int_{0}^{t^{\prime}} \exp \left(-\frac{2 t}{\tau_{M}}+\left(\frac{1}{\tau_{M}}-\frac{1}{\tau_{I}}\right) t^{\prime}+\left(\frac{1}{\tau_{M}}+\frac{1}{\tau_{I}}\right) t^{\prime \prime}\right) \mathrm{d} t^{\prime \prime}, \\
&= \frac{2 \sigma_{I}^{2}}{c^{2}} \cdot \frac{\tau_{M} \tau_{I}}{\tau_{M}+\tau_{I}}\left(\frac{\tau_{M}}{2}-\frac{\tau_{M}}{2} \exp \left(-\frac{2 t}{\tau_{M}}\right)\right. \\
&\left.-\frac{\tau_{M} \tau_{I}}{\tau_{I}-\tau_{M}}\left[\exp \left(-\frac{\tau_{M}+\tau_{I}}{\tau_{M} \tau_{I}} t\right)-\exp \left(-\frac{2 t}{\tau_{M}}\right)\right]\right) .
\end{aligned}
$$

The stationary solution is computed by taking the limit:

$$
t \rightarrow \infty, \quad \sigma_{V}^{2} \rightarrow \frac{\sigma_{I}^{2}}{c^{2}} \cdot \frac{\tau_{M}^{2} \tau_{I}}{\tau_{M}+\tau_{I}}
$$




\section{Correlation Function in Terms of Impulse Response}

More generally, in a linear stationary system, the response to any input $I(t)$ is given by the convolution of the input and the impulse response:

$$
V(t)=\int_{-\infty}^{\infty} G\left(t-t^{\prime}\right) I\left(t^{\prime}\right) \mathrm{d} t^{\prime}
$$

where $G=V(t)$ is the impulse response to $I(t)=\delta(t)$.

The auto-correlation function of $V(t)$ can be written as:

$$
\begin{aligned}
C_{v}(\tau) & =\langle V(0) V(\tau)\rangle, \\
& =\int_{-\infty}^{\infty} \int_{-\infty}^{\infty} G\left(0-t^{\prime}\right) G\left(\tau-t^{\prime \prime}\right)\left\langle I\left(t^{\prime}\right) I\left(t^{\prime \prime}\right)\right\rangle \mathrm{d} t^{\prime} \mathrm{d} t^{\prime \prime} \\
& =\int_{-\infty}^{\infty} \int_{-\infty}^{\infty} G\left(0-t^{\prime}\right) G\left(\tau-t^{\prime \prime}\right) C_{I}\left(t^{\prime}-t^{\prime \prime}\right) \mathrm{d} t^{\prime} \mathrm{d} t^{\prime \prime}
\end{aligned}
$$

Now we introduce new variables $s$ and $r$ :

$$
\begin{gathered}
s=t^{\prime}+t^{\prime \prime}, \quad r=t^{\prime}-t^{\prime \prime}, \\
t^{\prime}=\frac{1}{2}(s+r), \quad t^{\prime \prime}=\frac{1}{2}(s-r) . \\
\mathrm{d} t \mathrm{~d} t^{\prime \prime}=\left|\begin{array}{cc}
\frac{\partial t^{\prime}}{\partial s} & \frac{\partial t^{\prime}}{\partial r} \\
\frac{\partial t^{\prime \prime}}{\partial s} & \frac{\partial t^{\prime \prime}}{\partial r}
\end{array}\right| \mathrm{d} s \mathrm{~d} r=\left|\begin{array}{cc}
\frac{1}{2} & \frac{1}{2} \\
\frac{1}{2} & -\frac{1}{2}
\end{array}\right| \mathrm{d} s \mathrm{~d} r=-\frac{1}{2} \mathrm{~d} s \mathrm{~d} r . \\
C_{v}(\tau)=-\frac{1}{2} \int_{-\infty}^{\infty} \int_{-\infty}^{\infty} G\left(-\frac{1}{2}(s+r)\right) G\left(\tau-\frac{1}{2}(s-r)\right) C_{I}(r) \mathrm{d} s \mathrm{~d} r .
\end{gathered}
$$

If we define

$$
G^{(2)}(\tau, r)=-\frac{1}{2} \int_{-\infty}^{\infty} G\left(-\frac{1}{2}(s+r)\right) G\left(\tau-\frac{1}{2}(s-r)\right) \mathrm{d} s
$$

and use $t^{\prime \prime}$ instead of $s$ :

$$
\begin{aligned}
t^{\prime \prime} & =\frac{1}{2}(s-r), \quad \mathrm{d} t^{\prime \prime}=\frac{1}{2} \mathrm{~d} s, \\
s & =r+2 t^{\prime \prime}, \quad s+r=2 r+2 t^{\prime \prime} .
\end{aligned}
$$

Then

$$
\begin{aligned}
G^{(2)}(\tau, r) & =-\frac{1}{2} \int_{-\infty}^{\infty} G\left(-\frac{1}{2}(s-r)\right) G\left(\tau-\frac{1}{2}(s+r)\right) \mathrm{d} s \\
& =-\int_{-\infty}^{\infty} G\left(-r-t^{\prime \prime}\right) G\left(\tau-t^{\prime \prime}\right) \mathrm{d} t^{\prime \prime} \\
& =\int_{-\infty}^{\infty} G(t) G(\tau+r+t) \mathrm{d} t
\end{aligned}
$$


The resulting correlation function is:

$$
C_{v}(\tau)=\int_{-\infty}^{\infty} G^{(2)}(\tau+t) C_{I}(t) \mathrm{d} t
$$

where

$$
G^{(2)}(\tau+t)=\int_{-\infty}^{\infty} G\left(t^{\prime}\right) G\left(\tau+t+t^{\prime}\right) \mathrm{d} t^{\prime}
$$

\section{Subthreshold Response to Ornstein-Uhlenbeck Currents}

The response function $G(t)$ to an impulse $\delta(t)$ is derived from the linear equation:

$$
c \dot{V}=-g_{L} V+\delta(t)
$$

where we assume $V(t<0)=0$ and integrate both sides over a small interval $(-\varepsilon, \varepsilon)$ around zero:

$$
\begin{aligned}
\int_{-\varepsilon}^{\varepsilon} c \dot{V} \mathrm{~d} t & =-g_{L} \int_{-\varepsilon}^{\varepsilon} V(t) \mathrm{d} t+\int_{-\varepsilon}^{\varepsilon} \delta(t) \mathrm{d} t \\
c \Delta V & =o(\varepsilon)+1 \\
\Delta V & =\frac{1}{c} .
\end{aligned}
$$

Thus the impulse response can be written as:

$$
\begin{gathered}
G(t)=\frac{\theta(t)}{c} \exp \left(-\frac{t}{\tau_{M}}\right) . \\
G^{(2)}(\Delta)=\int_{-\infty}^{\infty} G\left(t^{\prime}\right) G\left(\Delta+t^{\prime}\right) \mathrm{d} t^{\prime} \\
=\frac{1}{c^{2}} \int_{-\infty}^{\infty} \theta\left(t^{\prime}\right) \theta\left(\Delta+t^{\prime}\right) \exp \left(-\frac{2 t^{\prime}+\Delta}{\tau_{M}}\right),
\end{gathered}
$$

where $\Delta=\tau+t$.

We rewrite $\mathrm{Eq} 9.15$ for $\Delta>0$ :

$$
G^{(2)}(\Delta)=\frac{1}{c^{2}} \int_{0}^{\infty} \exp \left(-\frac{2 t^{\prime}+\Delta}{\tau_{M}}\right)=\frac{\tau_{M}}{2 c^{2}} \exp \left(-\frac{\Delta}{\tau_{M}}\right)
$$

and for $\Delta<0$,

$$
G^{(2)}(\Delta)=\frac{1}{c^{2}} \int_{-\Delta}^{\infty} \exp \left(-\frac{2 t^{\prime}+\Delta}{\tau_{M}}\right)=\frac{\tau_{M}}{2 c^{2}} \exp \left(\frac{\Delta}{\tau_{M}}\right)
$$


The correlation function for an input of Ornstein-Uhlenbeck current is given by

$$
C_{I}(t)=\sigma_{I}^{2} \exp \left(-\frac{|t|}{\tau_{I}}\right)
$$

From Eq 9.12 the correlation function of the output MP

$$
\begin{aligned}
C_{v}(\tau) & =\int_{-\infty}^{\infty} G^{(2)}(\tau+t) C_{I}(t) \mathrm{d} t \\
& =\frac{\sigma_{I}^{2} \tau_{M}}{2 C^{2}} \int_{-\infty}^{\infty} \exp \left(\frac{-|\tau+t|}{\tau_{M}}\right) \exp \left(-\frac{|t|}{\tau_{I}}\right) \mathrm{d} t
\end{aligned}
$$

When $\tau>0$,

$$
\begin{aligned}
C_{v}(\tau)= & \frac{\sigma_{I}^{2} \tau_{M}}{2 c^{2}} \cdot\left[\int_{0}^{\infty} \exp \left(\frac{-\tau-t}{\tau_{M}}\right) \exp \left(\frac{-t}{\tau_{I}}\right) \mathrm{d} t\right. \\
& \left.+\int_{-\tau}^{0} \exp \left(\frac{-\tau-t}{\tau_{M}}\right) \exp \left(\frac{t}{\tau_{I}}\right) \mathrm{d} t+\int_{-\infty}^{-\tau} \exp \left(\frac{\tau+t}{\tau_{M}}\right) \exp \left(\frac{t}{\tau_{I}}\right) \mathrm{d} t\right], \\
= & \frac{\sigma_{I}^{2} \tau_{M}}{2 c^{2}} \cdot \tau_{M} \tau_{I}\left[\frac{\exp \left(\frac{-\tau}{\tau_{M}}\right)+\exp \left(\frac{-\tau}{\tau_{I}}\right)}{\tau_{M}+\tau_{I}}+\frac{\exp \left(\frac{-\tau}{\tau_{M}}\right)-\exp \left(\frac{-\tau}{\tau_{I}}\right)}{\tau_{M}-\tau_{I}}\right] .
\end{aligned}
$$

When $\tau<0$,

$$
C_{v}(\tau)=\frac{\sigma_{I}^{2} \tau_{M}}{2 c^{2}} \cdot \tau_{M} \tau_{I}\left[\frac{\exp \left(\frac{\tau}{\tau_{M}}\right)+\exp \left(\frac{\tau}{\tau_{I}}\right)}{\tau_{M}+\tau_{I}}+\frac{\exp \left(\frac{\tau}{\tau_{M}}\right)-\exp \left(\frac{\tau}{\tau_{I}}\right)}{\tau_{M}-\tau_{I}}\right] .
$$

Taken together, the result can be written as:

$$
C_{v}(\tau)=\frac{\sigma_{I}^{2} \tau_{M}^{2} \tau_{I}}{2 c^{2}} \cdot\left[\frac{\exp \left(-\frac{|\tau|}{\tau_{M}}\right)+\exp \left(-\frac{|\tau|}{\tau_{I}}\right)}{\tau_{M}+\tau_{I}}+\frac{\exp \left(-\frac{|\tau|}{\tau_{M}}\right)-\exp \left(-\frac{|\tau|}{\tau_{I}}\right)}{\tau_{M}-\tau_{I}}\right]
$$

The variance of MP at stationary state is obtained by taking $\tau=0$ :

$$
\sigma_{V}^{2}=\frac{\sigma_{I}^{2} \tau_{M}^{2} \tau_{I}}{c^{2}\left(\tau_{M}+\tau_{I}\right)}
$$

\subsection{Firing-rate Response to Noisy Inputs}

\subsubsection{Stationary Response Function}

Neurons fire action potentials (APs) when the membrane potential fluctuation is above a certain threshold level, which is probably the most fundamental function 
of neurons. The electric pulses of APs are actively regenerated while traveling along the axon for long distance communication. Action potential can often be considered as an 'all-or-none' event in the sense that not its concrete waveform but the firing frequency is sensitive to the strength of stimuli. In other words, sensory inputs are transformed into spike trains of different rates.

In this context a model neuron's response function is characterized by the function of firing rate given the amplitude of the injected currents called the $f-I$ curve. Moreover, in the presence of noisy synaptic input $I_{\text {syn }}(t)$ with a variance $\sigma_{I}$ and a temporal correlation $\tau_{I}(\mathrm{Eq}$ (9.6) $)$, the firing rate is a function of the mean current level $I_{0}$, and of $\sigma_{I}$ and $\tau_{I}$.
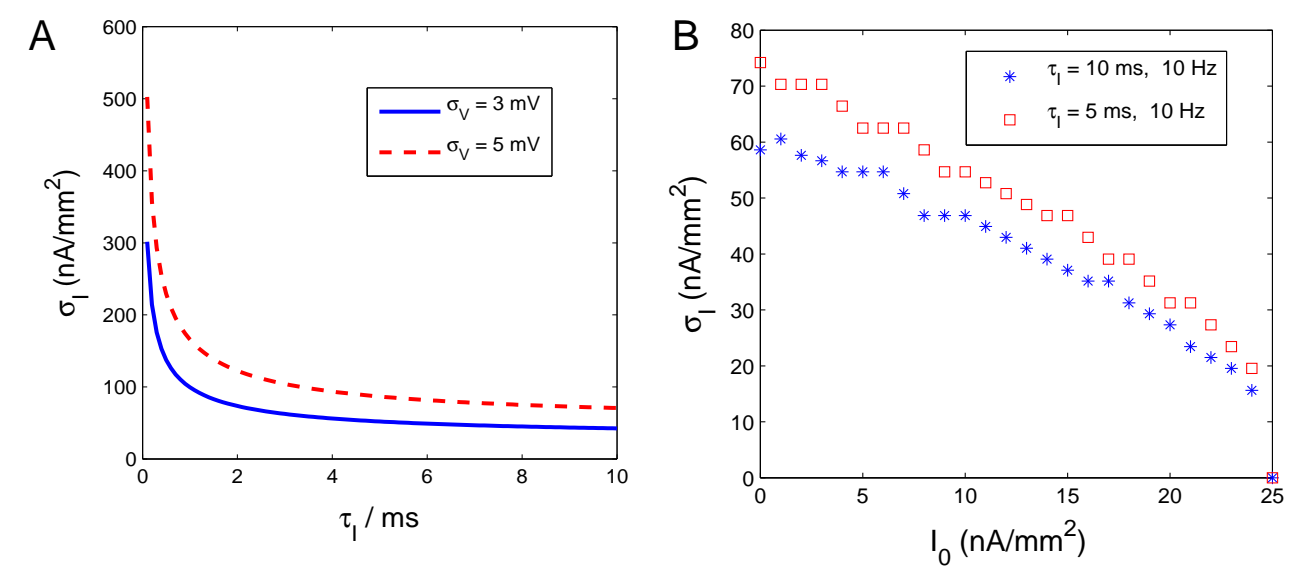

Figure 9.2: MP fluctuation and mean firing rates. (A) The contour lines of $\sigma_{V}=3 \mathrm{mV}$ (blue solid line) and $\sigma_{V}=5 \mathrm{mV}$ (red dashed line) on the $\tau_{I}-\sigma_{I}$ plane given by the analytical solution in Eq. 9.17 (B) The coutour lines of firing rate $\mathrm{f}=10 \mathrm{~Hz}$ in simulations varying $\sigma_{I}$ and $I_{0}$ simultaneously. See text for the detailed procedure of adjusting the input currents.

For our model to represent the stationary state behaviors of cortical neurons, we chose the input parameters of $\sigma_{I}$ and $\tau_{I}$ according to the subthreshold membrane potential fluctuation recorded in cortical neurons (Figure 9.3.1). In simulations we first chose $\tau_{I}$ according to the temporal correlation of MP fluctuations (Lampl et al., 1999). Next, $\sigma_{I}$ was determined from the analytical solutions obtained in Section 9.2 by setting the variance of the subthreshold membrane potential to $\sigma_{V} \simeq 3-5 \mathrm{mV}$ (Azouz \& Grav, 1999).

Finally, in order to keep the dynamics of our system in a low firing rate regime, we adjusted the constant current level $I_{0}$ to obtain a stationary firing rate of $10 \mathrm{~Hz}$ in all simulations. 


\subsubsection{Frequency Dependence of the Population Response}

One way to test a neuron's capability of tracking fast-changing stimuli is by measuring its response to temporally oscillatory inputs of different frequencies (Fourcaud-Trocmé \& Brunel, 2005; Naundorf et al., 2005b; Köndgen et al., 2008). Following these pioneering works, we revisit the frequency modulation function of the Wang-Buzsaki model in this section, to aid later comparison to cooperative gating models (Chapter 10) and to multi-compartment models (Chapter 12), and to the response functions recorded in cortical neurons (Köndgen et al., 2008).

The model neuron received an input consisting of constant current $I_{0}$, a sinusoidal input with a frequency of $f$ and a magnitude of $I_{1}$, and a synaptic noisy input $I_{\text {syn }}(t)$ of zero mean as discussed in Section 9.1.2.

$$
I(t)=I_{0}+I_{1} \sin 2 \pi f t+I_{\mathrm{syn}}(t)
$$

Figure 9.3 showed two simulation examples of neuronal responses to inputs
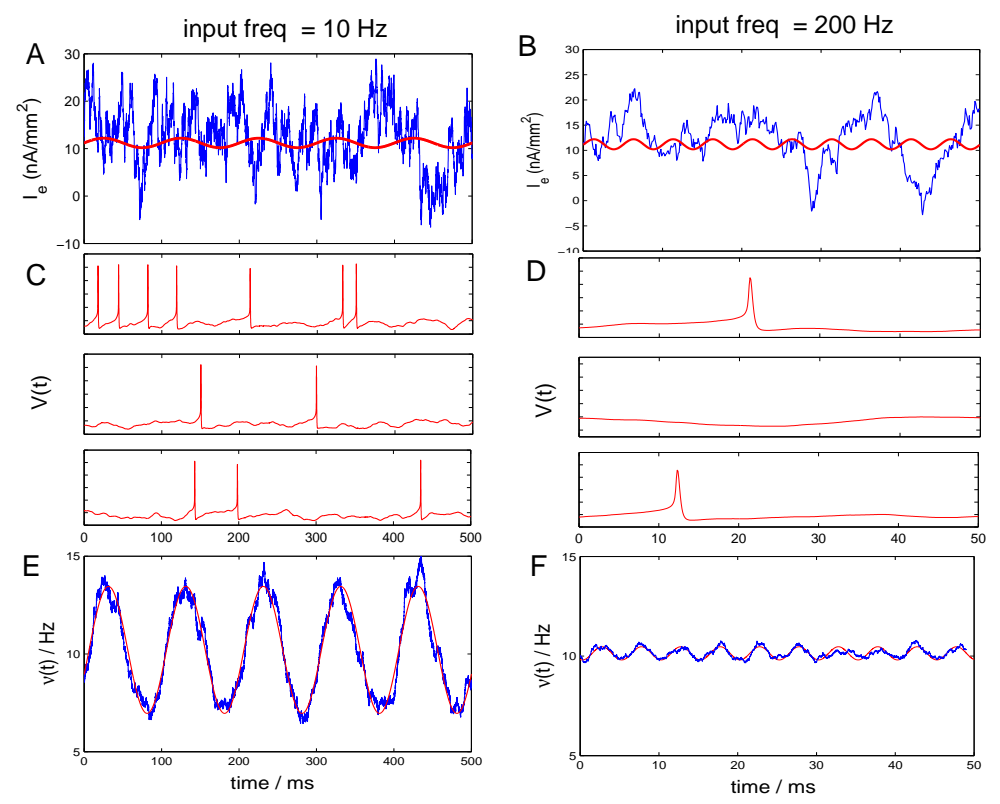

Figure 9.3: Neuronal response to noisy sinusoidal inputs (A,B) The injected currents (in blue) superimposed with a sinusoidal component (in red) of different frequencies, $\mathrm{f}=10 \mathrm{~Hz}$ and $\mathrm{f}=200 \mathrm{~Hz}$. (C,D) The voltage traces in three trials with different realizations of noisy synaptic inputs. (E,F) The instantaneous population firing rate estimated by the peristimulus time histogram(PSTH) across thousands of trials. It shows a much stronger modulation at input frequency of $\mathrm{f}=10 \mathrm{~Hz}$ compared to that of $\mathrm{f}=200 \mathrm{~Hz}$.

modulated at $\mathrm{f}=10 \mathrm{~Hz}$ and at $\mathrm{f}=200 \mathrm{~Hz}$. Although the mean firing rate of individual neurons remained constant at $\nu_{0}=10 \mathrm{~Hz}$, a large population of neurons 
was capable of tracking the input frequency up to $200 \mathrm{~Hz}$, albeit a strong damping of the modulation magnitude compared to $10 \mathrm{~Hz}$ frequency modulation.
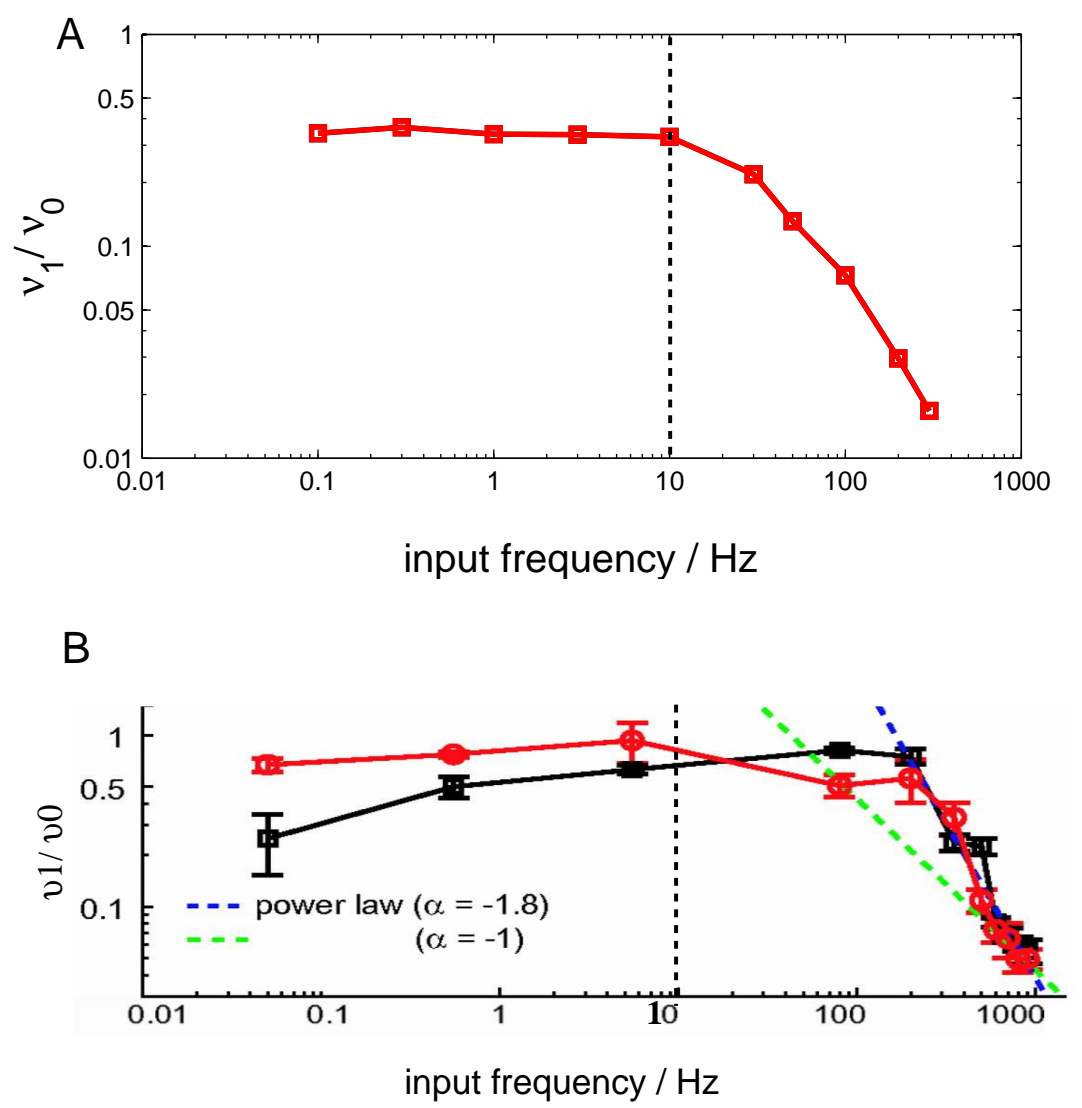

Figure 9.4: Frequency modulation of population firing rate (A) Modulation strength $\left(\nu_{1} / \nu_{0}\right)$ decayed with input frequency larger than $10 \mathrm{~Hz}$ in simulations of Wang-Buzsaki model. (B) Frequency response measured in cortical pyramidal neurons in response to sinusoidal noisv inputs (Adapted from Köndgen et al. (2008)). black: weak noise regime; red: strong noise regime. Dashed lines indicate the input frequency of $10 \mathrm{~Hz}$ for a better comparison.

The strength of the oscillatory response $\nu_{1}$ depends on the input frequency. As shown in Figure 9.3.2, the response amplitude of Wang-Buzsaki model started to attenuate when the input frequency was above $10 \mathrm{~Hz}$, which is the mean firing rate of individual neurons. This should be compared to the cut-off frequency (about $200 \mathrm{~Hz}$ ) of cortical neurons (Köndgen et al., 2008), which is much higher than that predicted by the model simulations. 


\subsubsection{Fast Response Properties with Fast AP Onset?}

Previous studies have suggested that the fast onset of AP generation would make the population response to transient signals faster (Fourcaud-Trocmé et al., 2003; Fourcaud-Trocmé \& Brunel, 2005; Naundorf et al., 2005a, 2006). In the following example we illustrate this idea by introducing an additional fast $\mathrm{Na}^{+}$channel components to the standard Wang-Buzsaki model and comparing the response speed of a neuronal population to a step change in the noisy input.
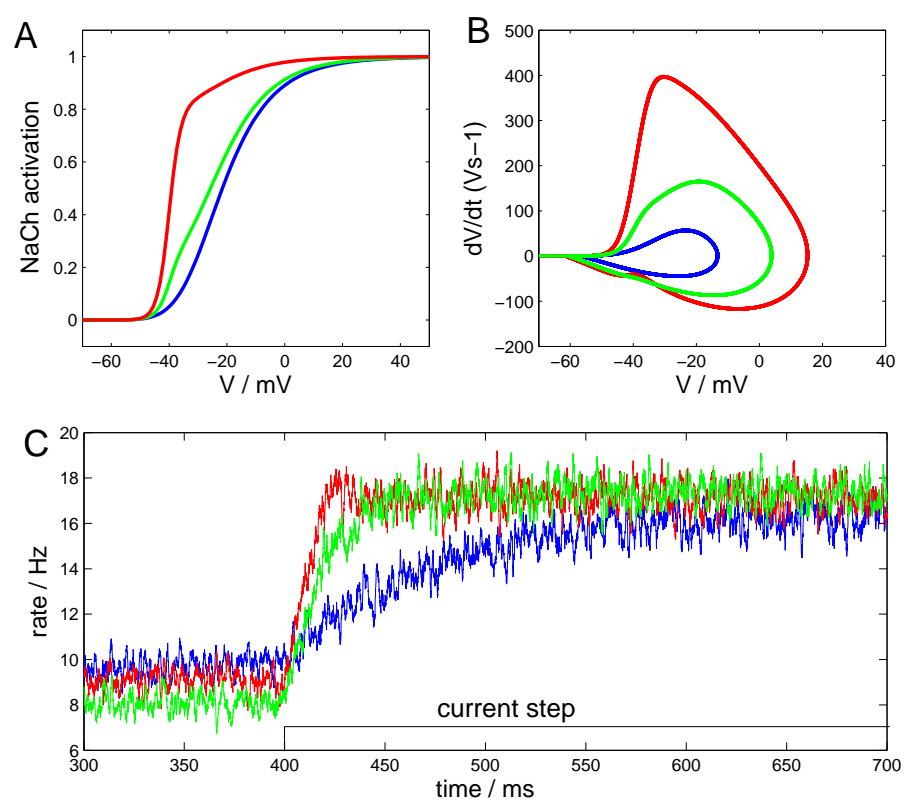

Figure 9.5: Fast response speed with fast $\mathrm{AP}$ onset (A) Activation curves of three models. blue: WB model with activation variable $m_{\mathrm{WB}}^{3}=m_{\infty}^{3}$; Green: A faster channel component with $m_{\text {fast }}=1 /(1+\exp (-V+40) / 2)$ was added into the WB model such that $m_{1}=0.8 m_{\mathrm{WB}}^{3}+0.2 m_{\text {fast }}$ (green); Red: $m_{2}=$ $0.8 m_{\mathrm{WB}}^{3}+0.2 m_{\mathrm{fast}}$. (B) Phase plot of the three models. $\bar{g}_{\mathrm{Na}}=300 \mathrm{pS} / \mathrm{\mu m}^{2}$. Faster channel kinetics result in larger peaks at $\dot{V}$. (C) Instantaneous population firing rates in response to a current step of the noisy input.

Figure 9.5A depicts the activation curves of the three models with different combinations of fast components and Wang-Buzsaki $\mathrm{Na}^{+}$currents. When the activation curves became steeper, the resulting action potentials exhibited faster onset with larger peak $\dot{V}$, which can be visualized by the phase plots shown in Figure 9.5B. We injected noisy currents into the three model neurons and computed the instantaneous firing rates. At time $t=400 \mathrm{~ms}$ a step function was applied to the constant current level of the noisy inputs. Figure $9.5 \mathrm{C}$ indicates that the model with faster AP onset dynamics responded faster to the transient signal. 
What is the physiological mechanism missing in the conventional conductance based models that makes them deviate from the fast response properties of real cortical neurons? Cooperative activation of $\mathrm{Na}^{+}$channels has been proposed by Naundorf et al. (2006) as a plausible mechanism for the fast onset dynamics of $\mathrm{AP}$ initiation and thus the fast response. In the next chapter we will construct the conductance based models with adjustable degree of channel cooperativity to examine the resulting AP waveforms and the corresponding impact on coding properties. 


\section{Appendix: Channel Kinetics of CB models}

In this thesis I explore two conductance based (CB) models of Hodgkin-Huxley type: the Wang-Buzsaki model (W-B, $m_{\infty}^{3} h-n^{4}$ ), and the Mainen-Sejnowski model $\left(\mathrm{M}-\mathrm{S}, m^{3} h-n\right)$. The equations of channel kinetics for both models are described in Table 9.1. The physiological parameters are summarized in Table 9.2 .

Table 9.1: Hodgkin-Huxleytype Models

\begin{tabular}{|c|l|l|}
\hline Model Name & Wang-Buzsaki $\left(m_{\infty}^{3} h-n^{4}\right)$ & Mainen-Sejnowski $\left(m^{3} h-n\right)$ \\
\hline \multirow{3}{*}{$\mathrm{Na}^{+}$channel } & $\alpha_{m}=\frac{0.1(V+35)}{1-\exp [-0.1(V+35)]}$ & $\alpha_{m}=\frac{0.182(V+35)}{1-\exp [-(V+35) / 9]}$ \\
& $\beta_{m}=4 \exp \frac{-(V+60)}{18}$ & $\beta_{m}=\frac{-0.124(V+35)}{1-\exp [-(V+35) / 9]}$ \\
& $\alpha_{h}=0.35 \exp \frac{-(V+58)}{20}$ & $\alpha_{h}=\frac{0.024(V+50)}{1-\exp [-(V+50) / 5]}$ \\
& $\beta_{h}=\frac{5}{1+\exp (-0.1(V+28))}$ & $\beta_{h}=\frac{-0.0091(V+75)}{1-\exp [-(V+75) / 5]}$ \\
\hline \hline \multirow{2}{*}{$\mathrm{K}^{+}$channel } & $m \equiv m_{\infty}:$ instantaneous & $h_{\infty}=\frac{1}{1+\exp [6.2(V+65)]}$ \\
& $\alpha_{n}=\frac{0.05(V+34)}{1-\exp (-0.1(V+34))}$ & $\alpha_{n}=\frac{0.02(V-20)}{1-\exp [-(V-20) / 9]}$ \\
& $\beta_{n}=0.625 \exp \frac{-(V+44)}{80}$ & $\beta_{n}=\frac{-0.002(V-20)}{1-\exp [-(V-20) / 9]}$ \\
\hline
\end{tabular}

Table 9.2: Physiological Parameters

\begin{tabular}{c|ccc} 
Parameter & symbol & Value & Unit \\
\hline Membrane capacitance & $\mathrm{C}_{\mathrm{m}}$ & 10 & $\mathrm{fF} / \mu \mathrm{m}$ \\
\hline Axial resistivity & $\mathrm{r}_{\mathrm{L}}$ & 1 & $\mathrm{M} \Omega \cdot \mu \mathrm{m}$ \\
\hline \multirow{3}{*}{ Reversal potential } & $\mathrm{E}_{\mathrm{L}}$ & -65 & $\mathrm{mV}$ \\
& $\mathrm{E}_{\mathrm{Na}}$ & 55 & $\mathrm{mV}$ \\
& $\mathrm{E}_{\mathrm{K}}$ & -90 & $\mathrm{mV}$ \\
\hline \multirow{3}{*}{ Channel density } & $\mathrm{g}_{\mathrm{L}}$ & 1 & $\mathrm{pS} / \mu \mathrm{m}^{2}$ \\
& $\overline{\mathrm{g}}_{\mathrm{Na}}$ & 350 & $\mathrm{pS} / \mu \mathrm{m}^{2}$ \\
& $\overline{\mathrm{g}}_{\mathrm{K}}$ & 150 & $\mathrm{pS} / \mu \mathrm{m}^{2}$ \\
\hline
\end{tabular}




\section{Chapter 10}

\section{Dynamic Response in Models with Cooperative Channel Gating}

\subsection{Introduction}

Voltage sensitive ion channels underly the information processing capabilities of nerve cells (Koch \& Segev, 1998; Davan \& Abbott, 2001; Hille, 2001). Ion channels are integral membrane proteins which depending on conformation can pass ionic currents and thus induce dynamic changes in transmembrane potentials (Hille, 2001). Neural and muscle cells use voltage sensitive ion channels as the fundamental nonlinear elements for electrical signaling. In these cells pulse-like electrical signals called action potentials (APs) are induced by an avalanche-like opening of channels.

Biophysical models for AP generation almost universally assume that individual channels open and close statistically independently and are coupled only through the transmembrane voltage. However, biological ion channels for a variety of physiologically important ions have been found capable of cooperative

gating when clustered (Schindler, 1984; Saito et al., 1988; Undrovinas et al., 1992; Marx et al., 1998; Molina et al., 2006). In cooperative gating the states of individual channels are not independent but coordinated such that the opening of one channel increases the opening probability of neighboring channels. Examples of cooperative gating have been found in $\mathrm{Na}^{+}$channels (Undrovinas et al., 1992), $\mathrm{K}^{+}$channels (Molina et al., 2006), $\mathrm{Ca}^{2+}$ channels (Marx et al., 1998) and in neurotransmitter receptors (Schindler, 1984). Fig. 10.1 shows the structure of clustered $\mathrm{Na}^{+}$and $\mathrm{Ca}^{2+}$ channels from electron microscopy. Intriguingly, patch recordings of such channels exhibit synchronized openings of double or triple channels.

Cooperative gating of ion channels has been proposed to represent a general capability of proteins to undergo so called 'conformational spread' (Brav \& Duke, 2004). Recently cooperative gating of $\mathrm{Na}^{+}$channels has been hypothesized to underly the observed rapid onset of APs in cortical neurons and to strongly 
influence the coding properties of cortical neurons (Naundorf et al., 2006).

(a)

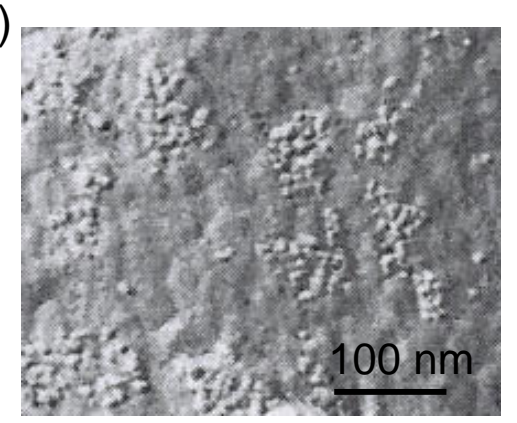

(b)
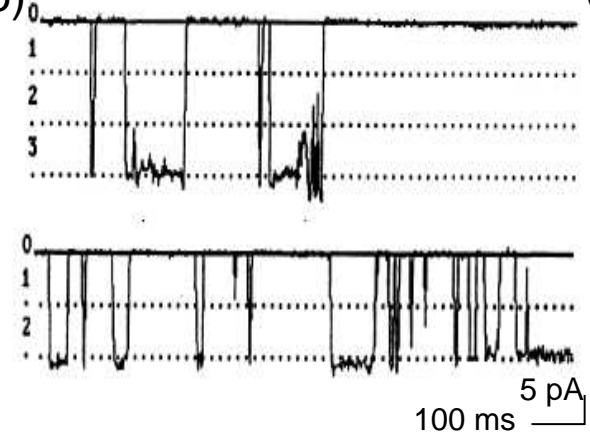

(c)

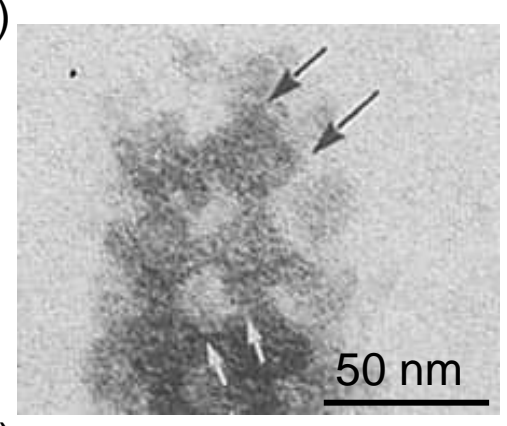

(d)
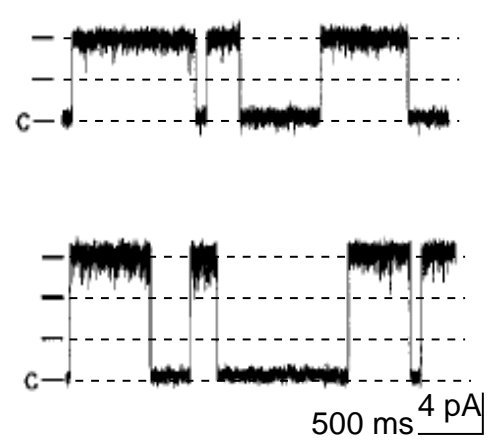

Figure 10.1: Channel clustering and cooperative gating in $\mathrm{Na}^{+}(\mathrm{a}, \mathrm{b})$ and $\mathrm{Ca}^{2+}$ channels (c,d). (a) Freeze-fracture electron microscopy reveals clustering of membrane particles in cardiac myocytes after ischaemia (Post et al., 1985). (b) Inside-out patch recordings of such cells showing simultaneous openings of pairs and triples of sodium channels (Undrovinas et al., 1992). (c) Transmission EM shows a dense crystalline array of RyR $\mathrm{Ca}^{2+}$ release channels in sarcoplasmic reticulum membrane (Saito et al., 1988). (d) Current traces through pairs and tripples of such channels exhibiting synchonized opening and closing (Marx et al., 1998). Dotted lines in (b, d) indicate single channel current steps.

Here we examine the dynamical and functional consequences of channel cooperativity in a conductance based model of neuronal AP generation in which a fraction $p$ of sodium channels exhibit cooperative gating. The model is constructed such that the strength of inter-channel coupling is quantified in voltage units and can be continuously varied between statistical independence and strong cooperativity.

We examine activation kinetics and AP waveforms predicted by the model for the entire range of cooperative channel fractions and coupling strengths. For strong cooperativity AP onsets become very rapid and for a small fraction of strongly cooperative channels APs exhibit a pronounced biphasic waveform often observed in nerve cells of the central nervous system (Eccles et al., 1958; Bean, 
2007). We point out that in this regime the AP onset is triggered by simultaneous opening of the cooperative channel fraction. We calculate the threshold for this synchronized opening and show that it depends on the fraction of noninactivated sodium channels. While increasing cooperativity lowers the threshold, the amount of threshold variability resulting from time varying levels of channel inactivation is largely insensitive to the strength of cooperativity.

To assess the functional impact of sodium channel cooperativity we characterize the ability of the neuronal firing rate to follow high frequency fluctuation in input current. Our results demonstrate that strongly cooperative sodium channel gating can boost the spike encoding of rapidly varying signals even if they represent only a small fraction of all sodium channels.

\subsection{AP Generator with Channel Cooperativity}

\section{Modeling Cooperative Gating of Sodium Channels}

To model cooperative gating of $\mathrm{Na}^{+}$channels, we assume that a channel in the cooperative population is coupled to $K$ neighboring channels such that the opening of each neighbor increases the probability of the channel to open. Using an activation variable $m(t)$ this is most simply realized by a kinetics of $m$ as

$$
\tau_{m}(V) \dot{m}^{J}(t)=m_{\infty}\left(V(t)+K J\left(m^{J}(t)\right)^{x} h\right)-m^{J}(t) .
$$

Here $m_{\infty}(V)$ is the steady state activation curve of individual channels, $\tau_{m}(V)$ is the activation time constant, $h$ is the available fraction, $\left(m^{J}(t)\right)^{x} h$ is the open probability so that $K h\left(m^{J}(t)\right)^{x}$ is the expected number of open neighbors. $J$ is a coupling constant in units of $\mathrm{mV}$ that measures the strength of coupling by the voltage shift that would increase the open probability of an isolated channel by the same amount. Eq. (10.1) represents the mean field approximation of cooperative channel gating among a coupled population in which opening of individual channels is modeled as a Makov process (Naundorf et al., 2006). In the limit of $J=0$, Eq. (10.1) reduces to the classical case of independent channel activation.

We used this approach to examine the predicted signature of channel cooperativity on the activation of a voltage clamped population of sodium channels. Assuming a fixed available fraction $H_{0}=1$, very short activation time constant $\tau_{m}(V)$ and a Bolzmannian single gate activation curve

$$
m_{\infty}(V)=\left[1+\exp \left(-\frac{V-V_{\frac{1}{2}}}{k_{A}}\right)\right]^{-1} .
$$

The fraction of open channels $m_{\infty}^{J}(V)$ after a voltage step called the collective activation curve satisfies the self-consistent equation

$$
m_{\infty}^{J}(V)=m_{\infty}\left(V+K J\left(m^{J}(t)\right)^{x} H_{0}\right) .
$$


For the following analysis we take $x=1$ as suggested by recent in vitro recordings of $\mathrm{Na}^{+}$currents in cortical neurons (Baranauskas \& Martina, 2006), where the activation time course was best fitted by a linear rising mono-exponential function.

\section{The Critical Coupling Strength}

The steady state solution of $m_{\infty}^{J}$ is obtained from the intersection points of the two curves $y=m$ and $y=f(m)$, where $f(m)$ is defined as

$$
f(m)=\frac{1}{1+\exp \left[-\left(V+\lambda m-V_{\frac{1}{2}}\right) / k_{A}\right]}
$$

with $\lambda=H_{0} K J$. Figure 10.2 shows how the curve of $y=f(m)$ changes as the parameters $\lambda$ and $V$ increase. It becomes steeper with increasing effective
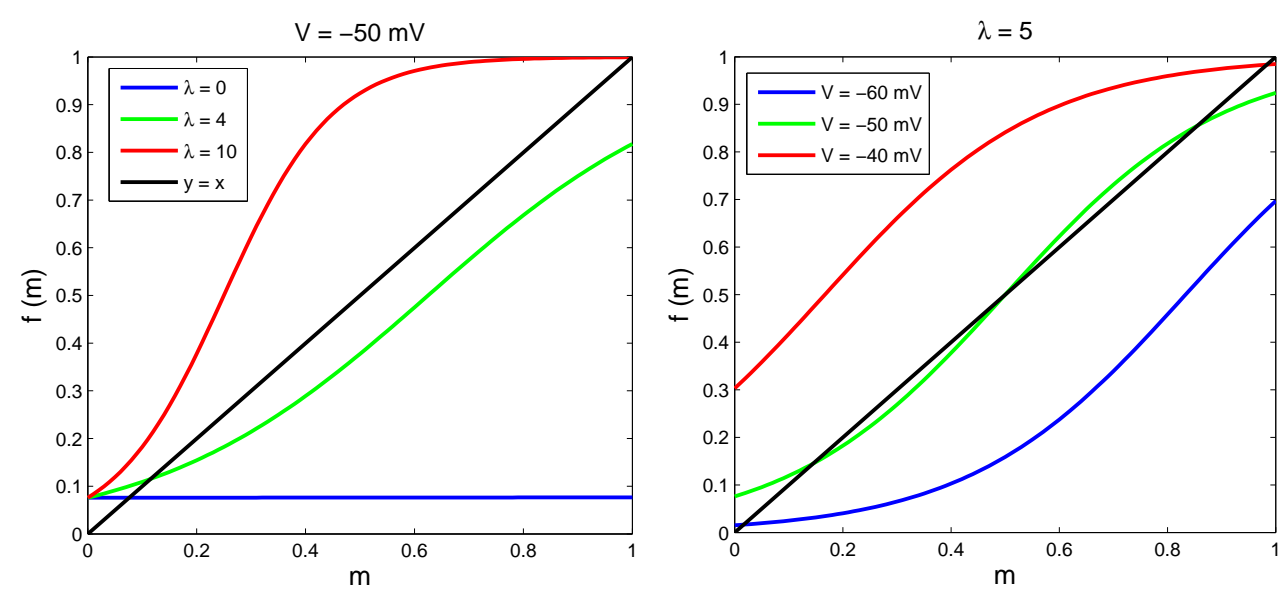

Figure 10.2: The steady state values of $m_{\infty}^{J}$ as intersection points of the two curves $y=m$ and $y=f(m)$. (A) The curve of $\mathrm{f}(\mathrm{m})$ is steeper as the coupling strength $\lambda$ increases. (B) The curve of $\mathrm{f}(\mathrm{m})$ is shifted to the left as $V$ increases.

coupling strength $\lambda$. When $\lambda$ is very small, there is only one intersection point at a small value of $m_{0}$ close to 0 . When $\lambda$ is very large, the intersection point shifts to a value close to 1 . In a critical range of $\lambda^{*}$, there exist three intersection points at certain ranges of voltages. By increasing $V$, the curve of $f(m)$ is shifted from right to left, thus a jump occurs from a small value of the fixed point $m_{0} \simeq 0$ to a larger value near 1 . At this transition point, the two curves of $y=f(m)$ and $y=m$ intersect tangentially. The critical value of $\lambda^{*}$ is thus obtained by solving the system

$$
\left\{\begin{array}{l}
F\left(m_{0}\right)=m_{0}+m_{0} A \exp \left(-\lambda m_{0}\right)-1=0 \\
\dot{F}\left(m_{0}\right)=1+A \exp \left(-\lambda m_{0}\right)-m_{0} A \lambda \exp \left(-\lambda m_{0}\right)=0
\end{array}\right.
$$


where

$$
A=\exp \left(-\frac{V-V_{\frac{1}{2}}}{k_{A}}\right), \quad \lambda=\frac{K J H_{0}}{k_{A}} .
$$

The solutions of $m_{0}$ are obtained from

$$
1-\lambda m_{0}+\lambda m_{0}^{2}=0
$$

which has real roots only if $\Delta=\lambda^{2}-4 \lambda \geq 0$. The value of $\lambda$ must be positive, hence $\lambda \geq 4$. The corresponding coupling strength has to satisfy

$$
J \geq \frac{4 k_{A}}{K H_{0}}
$$

to allow for a finite jump in collective activation curve.

\section{The Voltage Threshold}

Close to the transition point, the function of $f(m)$ can be approximated as an exponential expression of $m$ :

$$
f(m)=\frac{\exp \left[\left(V+\lambda m-V_{\frac{1}{2}}\right) / k_{A}\right]}{1+\exp \left[\left(V+\lambda m-V_{\frac{1}{2}}\right) / k_{A}\right]} \simeq \exp \left[\left(V+\lambda m-V_{\frac{1}{2}}\right) / k_{A}\right] .
$$

The critical value of $V^{*}$ is then obtained by solving the system

$$
\left\{\begin{array}{l}
G\left(m_{0}\right)=m_{0}-A \exp \left(\lambda m_{0}\right)=0 \\
\dot{G}\left(m_{0}\right)=1-A \lambda \exp \left(\lambda m_{0}\right)
\end{array}\right.
$$

where

$$
A=\exp \left(\frac{V-V_{\frac{1}{2}}}{k_{A}}\right), \quad \lambda=\frac{K J H_{0}}{k_{A}} .
$$

The solutions of $m_{0}$ and $A$ for a given $\lambda$ are

$$
m_{0}=\frac{1}{\lambda}, \quad A=\frac{1}{\lambda \exp (1)}
$$

and the corresponding threshold potential is

$$
V^{*}=-k_{A}(\ln \lambda+1)+V_{\frac{1}{2}}
$$


(a)

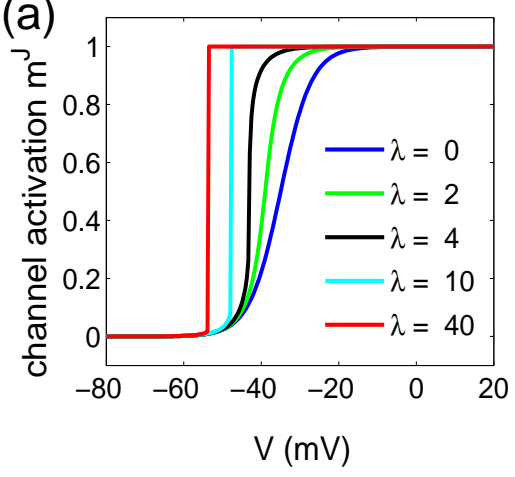

(c)

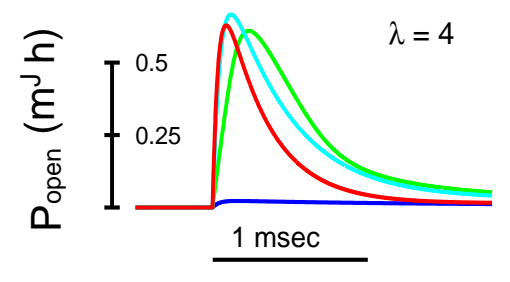

(b)

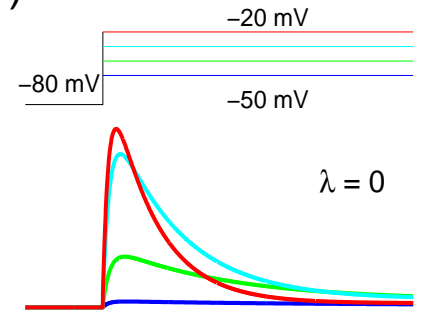

(d)

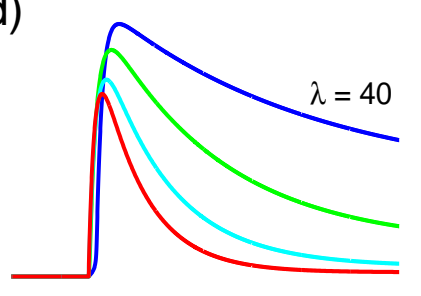

Figure 10.3: Dynamics of cooperative gating with varying degrees of coupling strength $\lambda\left(k_{A}=4, V_{1 / 2}=-35 \mathrm{mV}\right)$. (a) Collective activation curves for increasing strength of channel coupling. (b-d) Simulated open probability in response to a voltage-clamp protocol shown above the traces in (b). Currents are depicted for channel cooperativity smaller than, equal to, or greater than the critical value $\lambda^{*}=4$. 


\section{The Collective Activation Curve}

The shape of the collective activation curve obtained for different coupling constants depends only on the effective coupling strength $\lambda=K J H_{0} / k_{A}$ and is shown in Fig. 10.3 for different values of $\lambda$. For low values of $\lambda$ its slope increases with increasing $\lambda$ and at the critical effective coupling strength of $\lambda^{*}=4$ it develops a discontinuity at the threshold voltage $V^{*}$. For stronger coupling the threshold moves to more negative potentials and the collective activation curve approaches a step function such that almost all channels are closed below $V^{*}$ and almost all open above $V^{*}$.

This behavior was also apparent in simulated voltage clamp experiments, in which Eq. (10.1) was numerically solved assuming $\tau_{m}(V)=\phi /\left(\alpha_{m}(V)+\beta_{m}(V)\right)$ with the activation and deactivation rates functions and the kinetics of sodium channel inactivation as in the Wang-Buzsaki model (Wang \& Buzsáki, 1996) and $\phi=0.1$ to achieve a peak activation time constant of $50 \mu$ s as suggested by recent measurements of cortical sodium currents (Baranauskas \& Martina, 2006). In these simulations sodium channel opening above the critical coupling became basically an all or none event. Similar behavior was found for $x=3$ (data not shown).

\section{Conductance Based Models with Channel Cooperativity}

To assess the impact of sodium channel cooperativity on AP dynamics and encoding we included a fraction $p$ of cooperative sodium channels in a well characterized Hodgkin-Huxlev-tvpe neuron model (Wang \& Buzsáki. 1996: Fourcaud-Trocmé et al., 2003). In this cooperative WB model (cWB), the current balance equation reads as

$$
\begin{aligned}
& C \dot{V}(t)=g_{L}\left(V_{L}-V(t)\right)+\bar{g}_{K} n^{4}(t)\left(E_{K}-V(t)\right)+I_{\mathrm{ext}}(t) \\
& +\bar{g}_{\mathrm{Na}}\left[p\left(m^{J}(t)\right)^{x} h^{J}(t)+(1-p) m_{\infty}^{\prime 3} h^{\prime}(t)\right]\left(V_{N a}-V(t)\right),
\end{aligned}
$$

and the activation variable of the cooperative sodium channel fraction is given by Eq. (10.1). The inactivation curve of the cooperative channels $h_{\infty}^{J}$ has the same amount of voltage shift $h_{\infty}^{J}(V)=h_{\infty}^{\prime}\left(V+H_{0} K J m^{J}(V)\right)$ and $\tau_{h^{J}}(V)=$ $\tau_{h_{\infty}^{\prime}}\left(V+H_{0} K J m^{J}(V)\right)$ as the activation kinetics. All kinetic equations for the other gating variables are as in Chapter 9 (Wang \& Buzsáki, 1996).

\subsection{AP Waveforms and Onset Dynamics}

\section{Onset Rapidness and Bi-phasic APs in Phase Plots}

In this model, action potential waveforms as assessed by graphs of $\dot{V}$ vs. $V$ (phase plots) were very sensitive to the fraction of cooperative channels and the coupling 
strength (Fig. 10.4). In general the rapidness of AP onsets strongly increased with coupling strength. For a large fraction of cooperative channels this resulted in monophasic APs of rapid onset. For a small fraction of cooperative channels the AP waveform was typically biphasic (Fig. 10.4, Lower left panel). In both regimes AP onset was essentially determined by the activation of the cooperative fraction. Moreover, for a low fraction of strongly cooperative channels, the model reproduces the bi-phasic action potential dynamics frequently observed in neurons of the mammalian central nerves system.
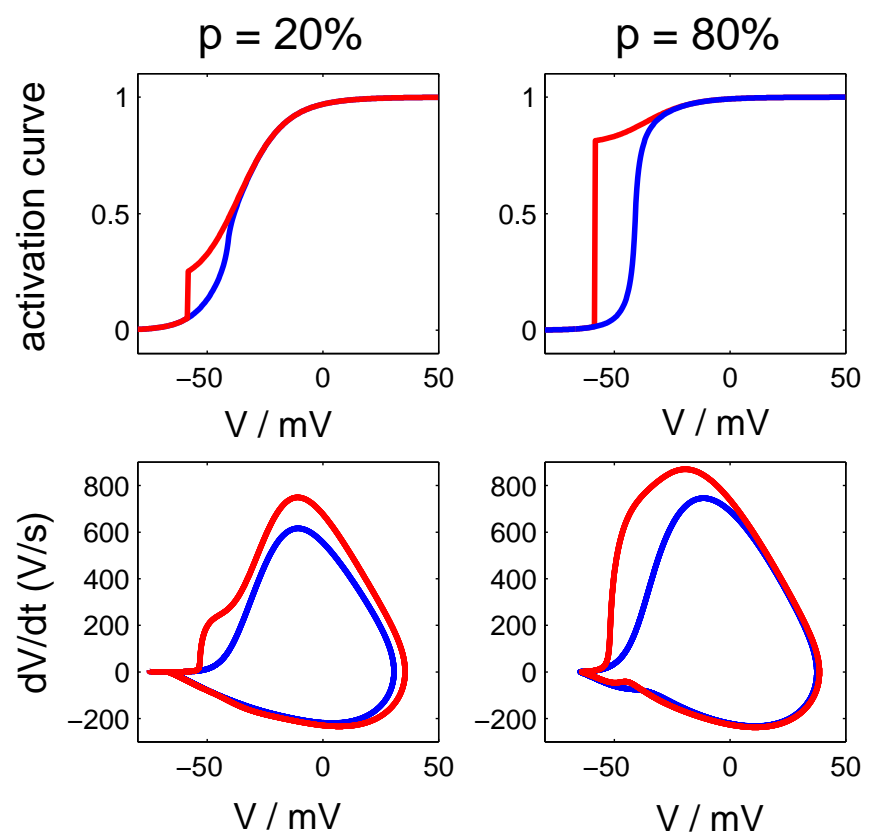

Figure 10.4: Channel activation and AP dynamics with small and large fractions of cooperative sodium channels. Upper panels: voltage dependence of total fraction of activated sodium channels; lower panels: phase plots of action potential generated by model Eq. (10.8). Blue: $K J=20 \mathrm{mV}$. Red: $K J=320 \mathrm{mV}$.

This behavior appears insensitive to detailed properties of model. For instance we found a similar dependence when we constructed the kinetics of the cooperative sodium channel fraction from $m_{\infty}(V)$ of the WB model instead of the Bolzmannian Eq. (10.2) and when we used an exponent $x=3$ that leads to a delayed activation of sodium channels. For this model, Fig. 10.5] shows the phase diagram of the AP waveforms. The onset dynamics of APs is quantified by the slope of the phase plot at the characteristic points of AP initiation. The onset rapidness increases monotonically with increasing coupling strength. With weak inter-channel coupling $(K J<200 \mathrm{mV})$ APs exhibit a gradual rising phase (inset panel, left); with strong coupling $(K J>400 \mathrm{mV})$ among a large fraction of channels APs show a steep rising phase (inset panel, top right); strong cou- 
pling among a small fraction of channels induces biphasic APs with a fast initial phase of cooperative activation followed by a slow rising phase of non-cooperative channel activation (inset panel, bottom right).

The phase diagram in Fig. 10.5 also indicates that a tight coupling between cooperative channels would be required to quantitatively reproduce experimentally observed AP waveforms. APs of cortical neurons under physiological conditions exhibit an onset rapidness of at least $20 / \mathrm{ms}$. The total coupling strength $K J$ needed to obtain such a rapid onset in our model is on the order of $400 \mathrm{mV}$ or more. Assuming for instance that each channel is coupled to roughly 10 neighbors this would imply that opening of a single neighboring channel is equivalent to a voltage shift by $40 \mathrm{mV}$. It is expected that such a strong inter-channel coupling would lead to highly synchronized gating such that the coupled clusters of channels behave as one effective functional unit. It is intriguing that all direct reports of coupled activation of ion channels have described exactly this type of highly synchronized channel opening and closing (Undrovinas et al., 1992; Molina et al., 2006; Marx et al., 1998; Schindler, 1984).

(a)

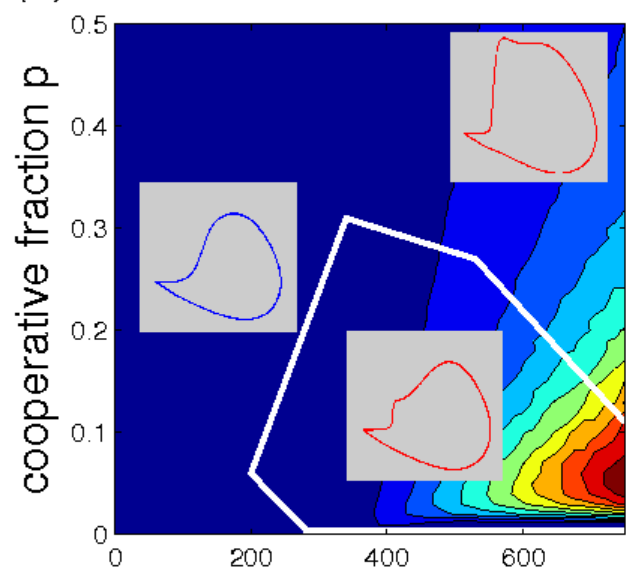

coupling strength $\mathrm{KJ}(\mathrm{mV})$ (b)

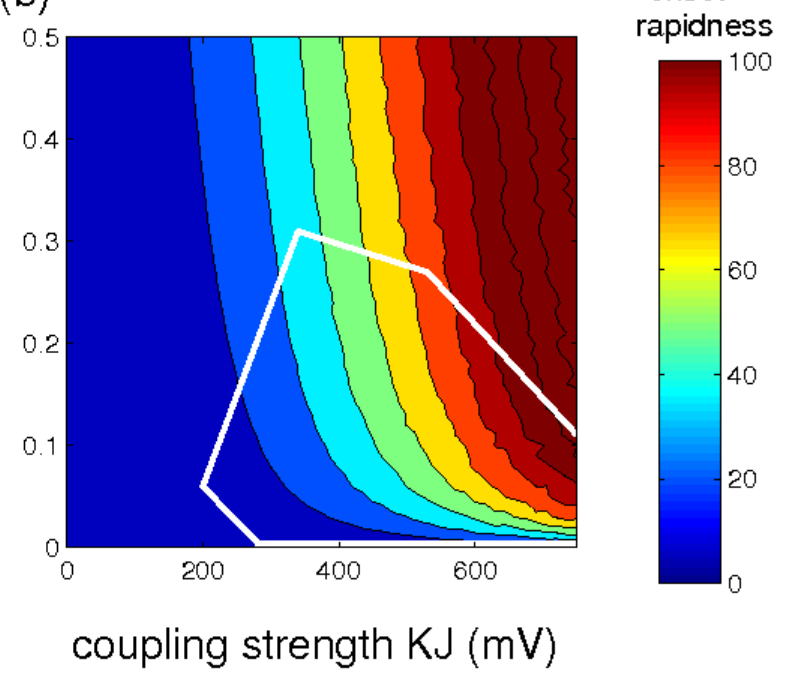

Figure 10.5: Phase diagram of the AP waveforms. white contour: bounder line between the monophasic and the biphasic APs (see insets); color code: onset rapidness measured as the phase slope at (a) $\mathrm{d} V / \mathrm{d} t=25 \mathrm{~V} / \mathrm{s}$ and at (b) the maximum second derivative $\mathrm{d}^{2} V / \mathrm{d} t^{2}$.

\section{Threshold Variability is Insensitive to the Coupling Strength}

In the presence of strong channel cooperativity, APs are initiated at the discontinuous jump of $m_{\infty}^{J}(V)$. As previously described in Eq. (10.7), the corresponding 
voltage threshold is given by

$$
V^{*} \simeq-k_{A} \log \left(H_{0}\right)-k_{A} \log \left(K J / k_{A}\right)-k_{A}+V_{\frac{1}{2}} .
$$

This relation implies that the threshold variability caused by different levels of $H_{0}$ is not affected by the coupling strength $K J$. We injected noisy synaptic currents to the model defined by Eq. (1-4) with different coupling strengthes. The phase plots of APs exhibit fast onset with strong inter-channel cooperativity and slow onset with weak coupling, whereas the threshold variability is largely unaffected (Fig. 10.6). In contrast, it has been shown in Naundorf et al. (2006) that the onset rapidness and threshold variability, as two characteristic features observed in APs of cortical neurons, are antagonistic in Hodgkin-Huxley-type models. Instead, our models of a cooperative AP generator illustrated a mechanism of fast onsets highly independent of threshold variability, as predicted by Naundorf et al. (2006).
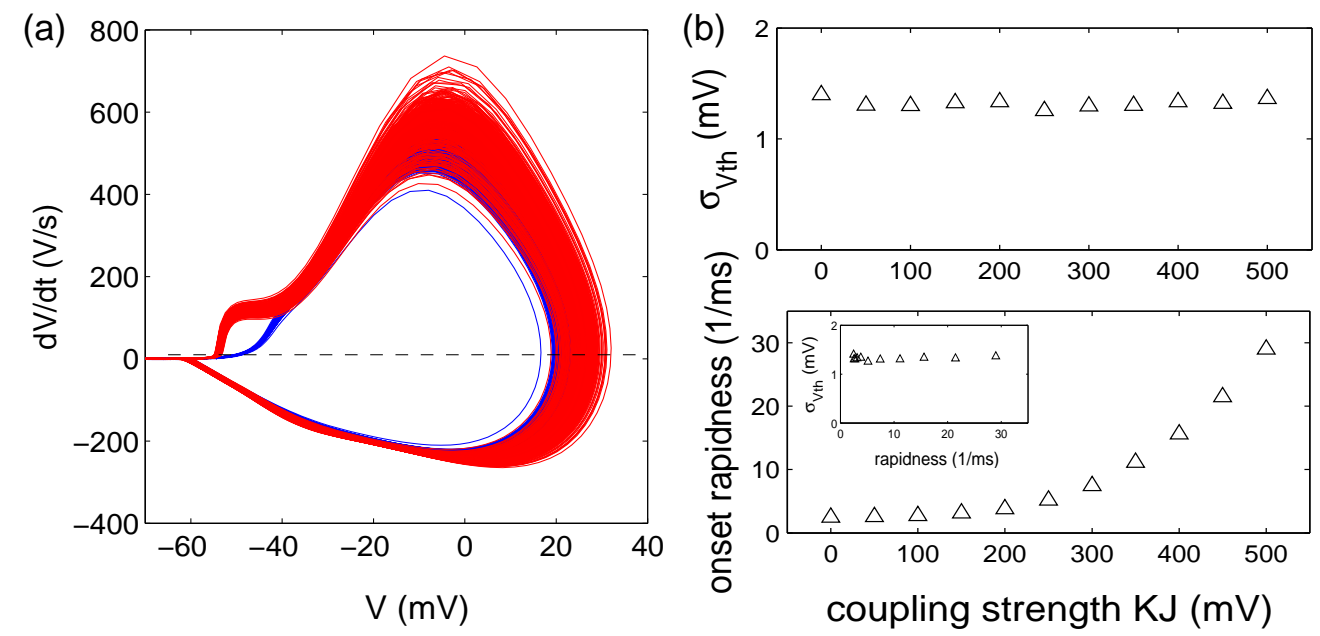

Figure 10.6: Threshold variability is insensitive to the strength of channel coupling. The model contains two populations of cooperative and non-cooperative $\mathrm{Na}^{+}$channels as those in Fig. 10.4. APs are generated by injection of the noisy synaptic current modeled as an Ornstein-Uhlenbeck process. (a) Phase plots of two models with $K J=100 \mathrm{mV}$ (blue trace) and $K J=400 \mathrm{mV}$ (red trace); $p=10 \%$. (b) The standard deviation of the voltage threshold $\sigma_{V_{\mathrm{th}}}$ varies only slightly with increasing coupling strength $K J$ from 0 to $500 \mathrm{mV}$ (upper panel); the onset rapidness of APs increases monotonically with $K J$ (lower panel); inset: $\sigma_{V_{\mathrm{th}}}$ as a flat function of onset rapidness.

\subsection{Impact on Dynamic Response Properties}

What are the coding properties of AP generators with cooperative channel gating? Following the approach introduced in the previous chapter, we characterized the 
firing rate dynamics in response to noisy oscillatory inputs of different frequencies. A time dependent current

$$
I(t)=I_{0}+I_{1} \cos (2 \pi f t)+\sigma_{I} I_{\mathrm{syn}}(t)
$$

was injected with the background synaptic noise $I_{\text {syn }}(t)$ generated from a stochastic Ornstein-Uhlenbeck process

$$
\tau_{c} \dot{I}_{\mathrm{syn}}=-I_{\mathrm{syn}}+\sqrt{1-\exp \left(-2 \Delta t / \tau_{c}\right)} \xi_{t},
$$

where $\xi_{t}$ is a Gaussian random variable. In linear response regime the instantaneous firing rate could be well approximated by

$$
\nu(t)=\nu_{0}+\nu_{1} \cos (2 \pi f t+\phi(f)) .
$$

In simulations $\nu_{0}$ was set to $10 \mathrm{~Hz}$ by adjusting the constant current level $I_{0}$. We graphed the modulation of the instantaneous firing rate against the input frequency.

As shown in Fig. 10.7, the high frequency modulation improves substantially with cooperative channel gating. The modulation amplitude at input frequency $f>200 \mathrm{~Hz}$ is almost one order of magnitude larger than that in the uncoupled model. For high frequencies the modulation gain decays roughly exponentially, which deviates somewhat from the power law behaviors previously reported for conventional conductance-based models (Knight, 1972a b; Brunel et al., 2001; Fourcaud-Trocmé et al., 2003; Naundorf et al., 2005a; Badel et al., 2008). In contrast, with all channels statistically independent, even a 10-fold increase of $\mathrm{Na}^{+}$channel density had no effects on the high frequency coding properties of the model although the peak rate of rise of APs was strongly increased. This result shows that a minor fraction of channels with cooperative gating can strongly affect the encoding properties of a neuronal AP generator.

\subsection{Summary and Discussion}

In summary, our analysis characterizes a new class of models for AP generation with cooperative channel gating. The AP waveforms of cortical neurons are known to exhibit fast onset dynamics and sometimes two distinct components in phase plots. In the past, this biphasic waveform was interpreted as lateral current caused by antidromic AP invasion from AIS to the soma (Eccles et al., 1958; Bean, 2007; Yu et al., 2008). Our study raises an alternative interpretation and suggests the possibility that the first phase is induced by activation of the cooperative channel fraction. Our results directly demonstrate that cooperativity even in a small fraction can strongly affect high frequency encoding. Intriguingly several recent studies suggest that cortical neurons can faithfully encode fluctuating inputs above $100 \mathrm{~Hz}$ (Silberberg et al., 2004; Köndgen et al., 2008). As 


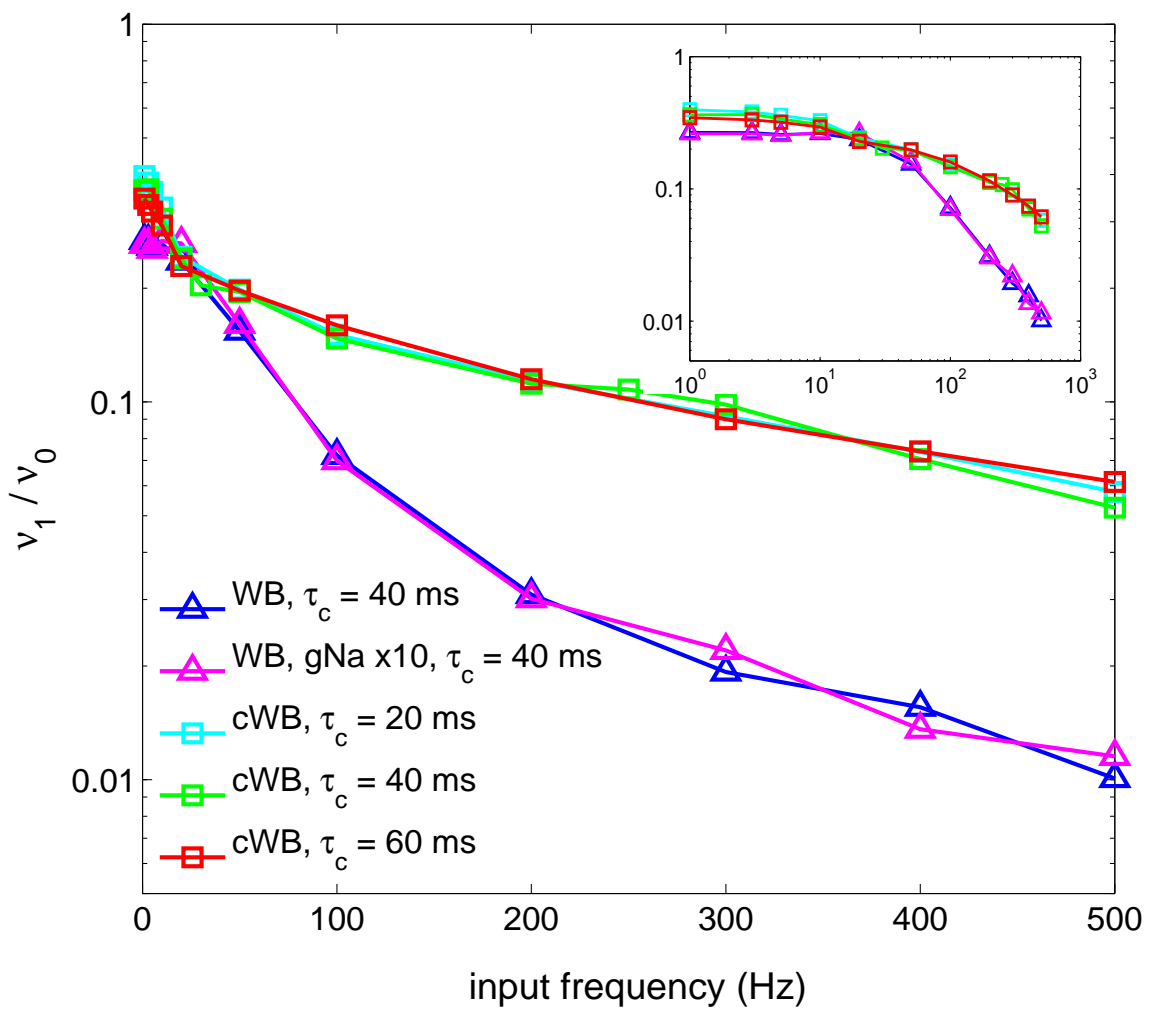

Figure 10.7: Cooperative channel activation improves the high-frequency response of a conductance-based model. Triangles: WB model; squares: WB model combined with $10 \%$ of cooperative $\mathrm{Na}^{+}$channels, $K J=1000 \mathrm{mV}$. Inset: data plotted in log-log scale. 
channel cooperativity has been directly observed in a wide variety of biological ion channels, it is an attractive hypothesis that it may contribute to high frequency sensitivity observed in these experiments.

So far direct evidence for channel cooperativity in cortical neurons is still missing. What is the potential mechanism of the strong coupling between cooperatively gated channels? First, there might be mechanical contact between channels through membrane associated proteins such as spectrin and ankyrin, whose conformational change would enforce a synchronized opening of neighboring channels. Second, the channels might interact through the local electric field where excess charges are brought in by opening of neighboring channels. Third, the voltage sensor of $\mathrm{Na}^{+}$channels not only detect the transmembrane electrical field. They also generate a field that may align the sensors of neighboring ion channels. Fourth, there might be $\mathrm{Na}^{+}$sensors remarkably sensitive to the influx of $\mathrm{Na}^{+}$ions. Finally, bilayer-mediated elastic forces has been proposed to drive conformational changes of transmembrane proteins cluster into cooperative groups (Ursell et al., 2007). Our study calls for the more detailed biophysical studies to uncover the mechanism behind strong inter-channel coupling.

On the other hand, an alternative mechanism for the fast somatic AP onset has been proposed by ( $\mathrm{Yu}$ et al., 2008), where they reported a smooth AP onset at axon initial segment (AIS) but a steep onset at soma, which was hypothesized to be caused by antidromic AP invasion from AIS to the soma. However, the use of axonal bleb recording was highly likely to destroy a tight coupling of channels if it exists in the neuronal membrane (Naundorf et al., 2007). In the following chapters, we will shift from point-neuron models to multi-compartmental models, to study the AP initiation and propagation in spatially extended neuron models and the coding properties of such models. 
106 Dynamic Response in Models with Cooperative Channel Gating 


\section{Chapter 11}

\section{AP Propagation and Neuron Geometry}

\section{$11.1 \quad$ Introduction}

In the previous chapter, we have studied the impact of cooperative gating of sodium channels on the dynamics and coding properties of neuronal action potentials. The cWB model with strong channel cooperativity is capable of reproducing the biphasic AP waveforms and the fast onset dynamics observed in cortical neurons. However, the observed shape of somatic APs in the cortex may be biased by antidromic AP invasion of the soma, as has been proposed by McCormick et al. (2007) and Yu et al. (2008). These authors claim that spike initiated at the axon initial segment(AIS) rises as smoothly as described by the Hodgkin-Huxley-type model; due to spatial inhomogeneity and the large lateral current supplied by the axonal spike, the AP back-propagating into soma results in a rapid rising phase. If this so-called 'lateral current hypothesis' is true, the fast AP onset at soma accompanied by a slow rise at the initiation site would not be expected lead to the ability of neuronal population to follow fast signal reliably.

Although there have been extensive studies on AP propagation in the literature (Hodgkin \& Huxlev, 1952; Jack et al., 1975; Rall, 1977; Meeks et al., 2005; Grill et al., 2007), they mainly focus on the reliability and velocity of propagation. The concrete waveforms of APs have received little attention except that in an early work by Goldstein \& Rall (1974) in which it was stated that the peak height of APs are reduced by flare of cable diameter.

We thus re-address the question: how is AP waveform, especially its onset rapidness, changed by propagation and by neuron geometry and in particular by the influence of a thin axon on a large soma? Before we discuss and implement the detailed physiological parameters and sometimes contradicting recordings of the neuronal channel distribution and electrophysiological properties, we first study 
the propagating AP in a geometric model with homogenous channel properties.

\subsection{Methods}

\subsubsection{Spatiotemporal Dynamics}

The spatial-temporal dynamics of signal propagtion in a neuron is mathematically described by the cable equation (for classical text books see Jack et al. (1975); Rall (1977); Davan \& Abbott (2001))

$$
c_{m} \frac{\partial V}{\partial t}=\frac{a}{2 r_{L}} \frac{\partial^{2} V}{\partial x^{2}}-i_{m}+i_{e}
$$

for a segment of cable of radius $a$ and intracellular resistivity $r_{L}$. The electrode current $i_{e}$ and the membrane current $i_{m}$ are expressed as currents per unit area of membrane. By convention the inward membrane currents have negative sign.

Since the kinetics of voltage-gated channels and synaptic conductances often induce a highly nonlinear behavior, they are neglected at the first step of analysis. One can make a linear approximation near the resting potential of the neuron, where the membrane current $i_{m}$ is replaced by product of the membrane potential and the leak conductance $g_{L} V$. A convenient expression is obtained by multiplying both sides of the equation by $r_{m}=1 / g_{L}$ :

$$
\tau_{M} \frac{\partial V}{\partial t}=\lambda^{2} \frac{\partial^{2} V}{\partial x^{2}}-V+r_{m} i_{e} .
$$

This expression shows that the membrane time constant $\tau_{M}=r_{m} c_{m}$ sets a scale of the temporal dynamics and a length constant $\lambda$ scales the spatial diffusion of the membrane potential

$$
\lambda=\sqrt{\frac{a r_{m}}{2 r_{L}}},
$$

\section{Time constants in a spatially extended neuron}

The membrane time constant $\tau_{M}$ sets the time scale of subthreshold membrane potential (MP) variation. The MP in an iso-potential patch of membrane responses to a current step by an exponential growth towards the steady state value:

$$
V(t)=V_{\infty}\left(1-\exp -t / \tau_{M}\right)
$$

However, a different rule of time evolution has been found in neurons with spatially extended structure, as suggested by the solution of the linear cable equation (Jack et al. (1975), first solved by Hodgkin \& Rushton (1946)). In response to a current step, the voltage change in an infinite cable at the site of current injection is governed by

$$
V(t)=V_{\infty} \times \operatorname{erf}\left(\sqrt{t / \tau_{M}}\right)
$$


The different time windows in the response of a infinite cable and a point neuron

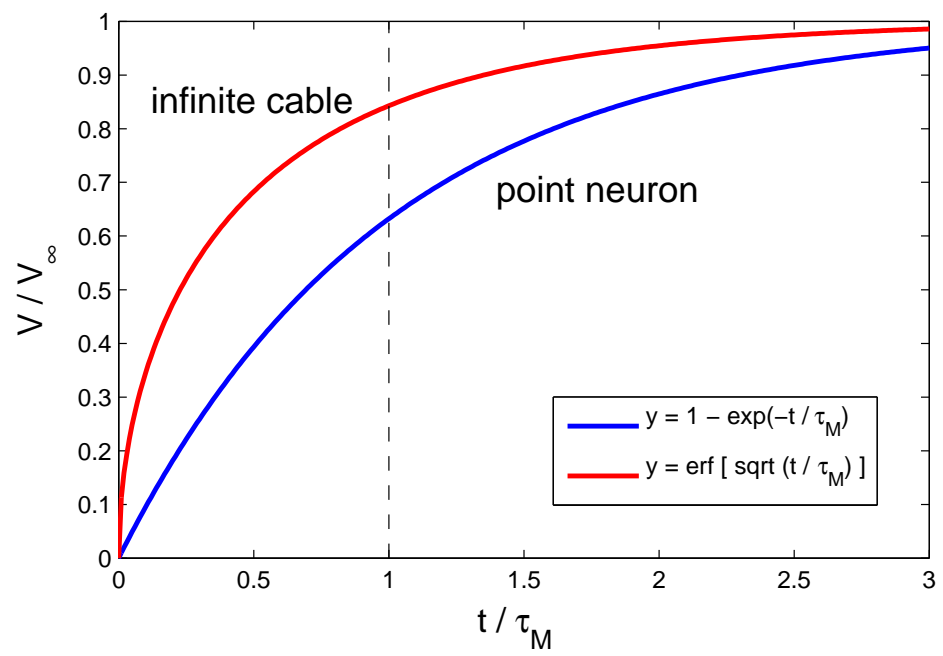

Figure 11.1: Voltage dynamics in a infinite cable and in a point neuron. In response to a current step at $\mathrm{t}=0$, the MP at the current injection site of an infinite cable rises faster to its steady state value (Eq. 11.5) than the exponential growth in an iso-potential patch of membrane (Eq. 11.4). Voltage is normalized to its steady state value and time is measured in units of membrane time constant $\tau_{M}$.

is depicted in Figure 11.1. Intuitively, the model of point neuron is equivalent to an iso-potential system of a uniform structure with constant current injection at every location. The same total amount of current caused a faster voltage rise at the injection site of the cable. In a realistic system of a finite cable, its temporal dynamics is then dependent on the spatial extension of the system.

\section{Length constant in modeling neuron morphology}

In a passive cable the changes of membrane potentials induced by a localized current injection decay back to zero over a distance of the same order as the electrotonic length constant $\lambda$.

From Eq. 11.3 the value of $\lambda$ is determined by the neuron's morphology (the radius $a$ ) and its physiological properties such as membrane resistance $r_{m}$ and the intracellular resistivity $r_{L}$. An experimental estimation yield a value of $\lambda \simeq$ $400 \mathrm{\mu m}$ in cortical neurons (Shu et al., 2006).

The length constant $\lambda$ sets a spatial scale for modeling of neuronal morphology. For example, a neurite much longer than $\lambda$ represents the behavior of an infinite cable whereas early terminations of axon and dendrite are expected to influence the voltage response at the soma. 
To track the spatial-temporal dynamics in simulations, the spatial resolution should be much smaller than the length constant $\lambda$, especially in simulations of a soma with varying geometry and of the transition zone of two adjancent parts with different physiological properties.

\subsubsection{Model Neuron Geometry}

In the following, the soma geometry is modeled as a sphere or an ellipsoid with a long and narrow axon going through one of the main axis of the ellipsoid (Figure 11.2). In this simplified model, the expansion of the soma from a homogenous cable can be quantitatively controlled, as described by the soma size factor $\alpha$, which measures the expansion ratio with respect to the length of the soma, such that the $\alpha$ value of zero indicates a homogenous cylinder, the value of one stands for a spherical soma and other values between zero and two represent ellipsoids.

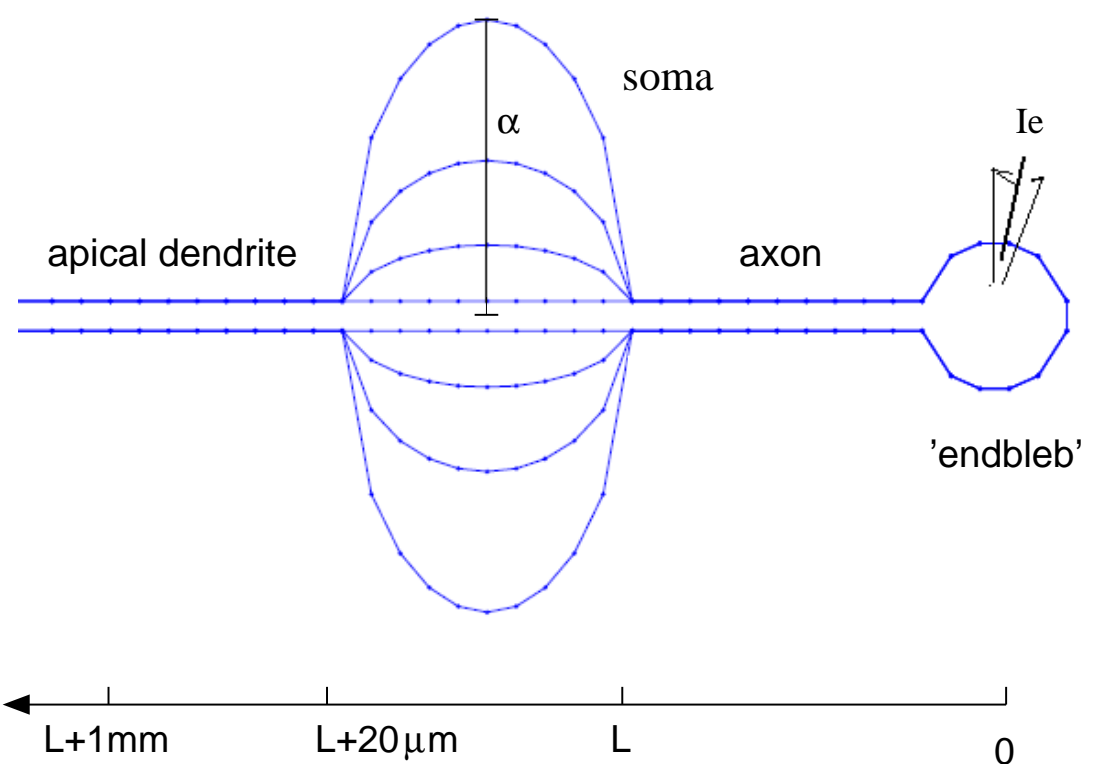

Figure 11.2: Morphology of the geometric model The distance from current injection site at axon to soma is measured by L, ranging from 0 to $2 \mathrm{~mm}$. The soma size factor $\alpha$ ranges between 0 and 2. $\alpha=0$ : cylinder; $\alpha=1$ : sphere; $\alpha=2$ : ellipsoid. The $\mathrm{Na}^{+}$channel density $\bar{g}_{N a}$ is assumed to be constant across the entire neuron, which was adjusted between 300 to $3000 \mathrm{pS} / \mathrm{\mu m}^{2}$ in simulations.

The AP propagation within the neuron is modeled by the cable equation (Eq11.1, for the discrete dynamical equations see Davan \& Abbott (2001)), where the membrane current $i_{m}$ consists of leak current, voltage-gated $\mathrm{Na}^{+}$and 
$\mathrm{K}^{+}$currents. The channel kinetics and physiological parameters are described by the Wang-Buzsaki model.

To account for the effect of propagation and spatial geometry per se, the electrical properties of the entire neuron were set to be uniform, as summarized in Table 9.2 of Chapter 9. Other conductance-based neuron models are discussed later. Detailed descriptions of different model kinetics can be found in Appendix of Chapter 9 .

To study the lateral current invasion from the axon into the soma, we induced antidromic APs at the end of the axon (modeled as an 'active bleb' in Figure 11.21). The propagating AP waveforms were studied in simulations systematically varying the distance from the injection site to the soma ( $L$, ranging from 0 to $2 \mathrm{~mm}$ ), the soma size factor ( $\alpha$, ranging from 0 to 2 ), and the sodium channel density $\left(\bar{g}_{N a}\right.$, ranging from 300 to $\left.3000 \mathrm{pS} / \mathrm{\mu m}^{2}\right)$.

\subsubsection{Computational Methods}

The model neuron (Figure 11.2) consists of the axonal endbleb, the distal axon (10 $\mu \mathrm{m}$ of segment length; $1 \mu \mathrm{m}$ in diameter), the proximal axon(1 $\mu \mathrm{m}$ of segment length; $1 \mu \mathrm{m}$ in diameter; 20 segments), the soma region(1 $\mu \mathrm{m}$ of segment length, 10 segments), the proximal dendrite (1 $\mathrm{mm}$ of segment length; $1 \mu \mathrm{m}$ in diameter; 10 segments), and the distal dendrite (20 $\mathrm{m}$ of segment length; $1 \mu \mathrm{m}$ in diameter; 50 segments). The number of the axonal segments ranging from one single compartment to 209 compartments, was adjusted according to the axon length $L$.

The geometry of the soma was modeled as an ellipse along the axonal axis. The soma diameter was obtained from the elliptic equation:

$$
\frac{x^{2}}{a^{2}}+\frac{y^{2}}{b^{2}}=1,
$$

where $a$ denotes the half length along the axonal axis and $b=\alpha L_{s} / 2$ is the half length in the perpendicular direction, which is controlled by the parameter $\alpha$. If we denote $L_{s}$ as the length of the soma and $r_{a}$ as the radius of the axon, then the soma meets the axon at the point with coordinates $\left(L_{s} / 2, r_{a}\right)$. By plugging the coordinates into Eq. 11.6. we get $a^{2}$ as a function of b:

$$
a^{2}=\frac{L_{s} \cdot b^{2}}{b^{2}-r_{a}} .
$$

Taken together, at any coordinate $\mathrm{x}$ of the point in the soma region, the corresponding y coordinate on the ellipse can be found by the formula:

$$
y^{2}=b^{2}-\frac{x^{2}\left(b^{2}-r_{a}^{2}\right)}{L_{s}^{2}} .
$$


A good spatial discretization was achieved by cone approximation instead of cylinder approximation of each compartment so that different discretization degrees would not change the overall structure of the neuron geometry.

Antidromic action potentials were triggered in simulations by constant current injection at the end of the axon. The current level was adjusted to get a constant firing rate of 10 Hzunder different parameter choices.

Simulations were performed using a $\mathrm{C}++$ program and all the figures were generated by user-specified Matlab functions. The dynamics of the system was solved by an implicit backward Euler method with a time step of $10 \mu \mathrm{s}$, which corresponds to a sampling frequency of $100 \mathrm{kiloHz}$.

\subsection{Simulation Results}

Figure 11.3 shows two examples of the propagating wave patterns. The AP waveforms at the initiation site were broader with smaller amplitudes. By propagation they became narrower and steeper until an invariant waveform was reached. Accordingly, on the phase plane representation (Figure 11.3 A2, B2), the limit cycles of the dynamics at subsequent locations expands until a linear phase is approached, which is corresponding to the exponential growth of the membrane potentials.

For comparison with the experimental recordings, we determined the point of AP onset at which the MP rate of change $d V / d t$ reached a value of $10 \mathrm{mV} / \mathrm{ms}$. Figure 11.3 A3 and Figure 11.3 B3 plot the onset latency against the propagation distance. The reciprocal of the slope on the latency-distance plot gives an estimation of the propagation velocity, which is about $0.6 \mathrm{~m} / \mathrm{s}$ with $g_{\mathrm{Na}}=300 \mathrm{pS} / \mathrm{\mu m}^{-2}$, and $1.0 \mathrm{~m} / \mathrm{s}$ with $g_{\mathrm{Na}}=1200 \mathrm{pS} / \mathrm{\mu m}^{-2}$.

The onset rapidness was measured by the slope of the phase plot $\dot{V}-V$ at the point of AP onset $\dot{V}=10 \mathrm{~ms}^{-1}$. While propagating away from the initiation site, the onset rapidness showed an 8-fold increase with low $\mathrm{Na}^{+}$channel density (Figure 11.3 A4) and a 20-fold increase with high $\mathrm{Na}^{+}$channel density (Figure 11.3 B4). However, if the soma was close to the initiation site (red circle, $\mathrm{L}=50 \mathrm{\mu m}$ ), the $\mathrm{AP}$ onset rapidness was as low as $3 \mathrm{~ms}^{-1}$. The effect of propagation became prominent only after a certain distance, in the order of one electrotonic length $(\lambda \simeq 400 \mu \mathrm{m})$.

To clarify the dependence on the various geometric and physiological parameters, we performed extensive simulations with the model varying distance $L$, soma size $\alpha$ and the maximum $\mathrm{Na}^{+}$conductance $\bar{g}_{\mathrm{Na}}$. For each simulation we computed the onset rapidness of the somatic AP, and plotted it in Figure 11.4 with respect to the three parameters.

The propagating APs exhibited a transition from the initial slow onset to a rapid onset (Figure 11.4 A). As the $\mathrm{Na}^{+}$channel density increased, the transition point was shifted closer to the AP initiation site, while the value of onset rapidness 

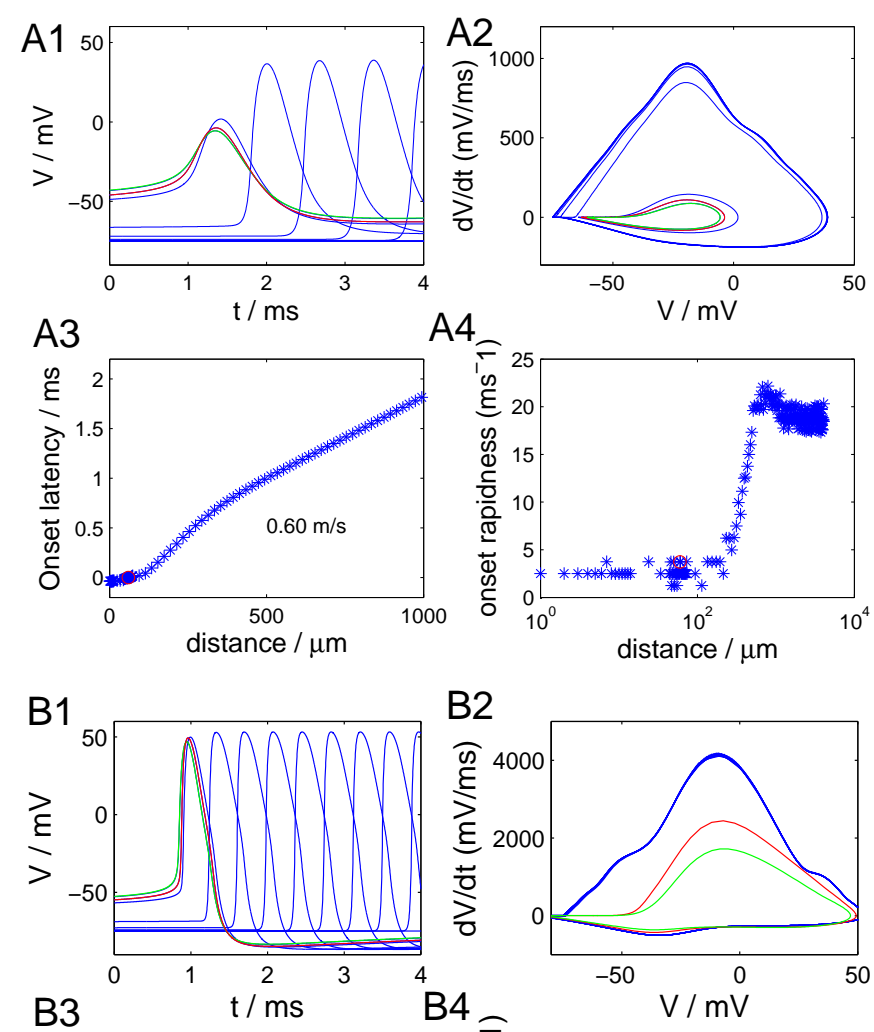

B2
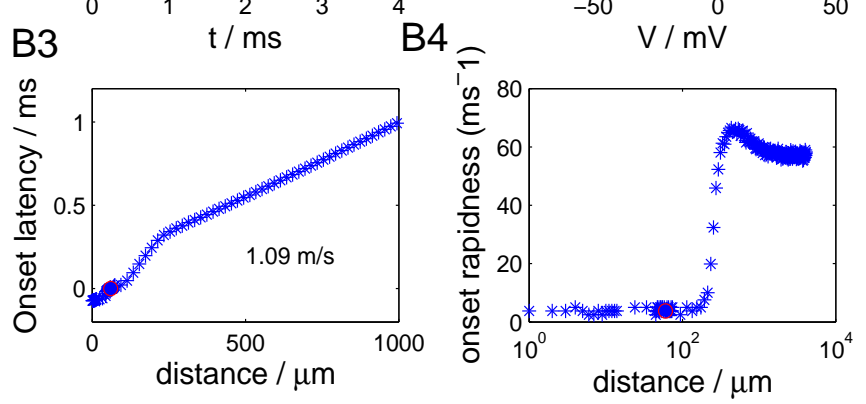

Figure 11.3: Two simulation examples in the geometric model (A) $L=$ $50 \mu \mathrm{m}, \alpha=1, \bar{g}_{\mathrm{Na}}=300 \mathrm{pS} / \mu^{2}$. (B) $L=50 \mu \mathrm{m}, \alpha=1, \bar{g}_{\mathrm{Na}}=1200 \mathrm{pS} / \mu^{2}$. (A1, B1) APs evoked at different locations are superimposed in time. Green trace: current injection site at the axon end; Red trace: soma; Blue: dendrite. (A2, B2) Phase plane representation: the rates of change of the membrane potential are computed for each location. (A3, B3) The AP onset latency plotted over distance. The point of AP onset is defined as $\mathrm{d} V / \mathrm{d} t=10 \mathrm{~V} / \mathrm{s}$. (A4, B4) The onset rapidness of APs at different locations. 


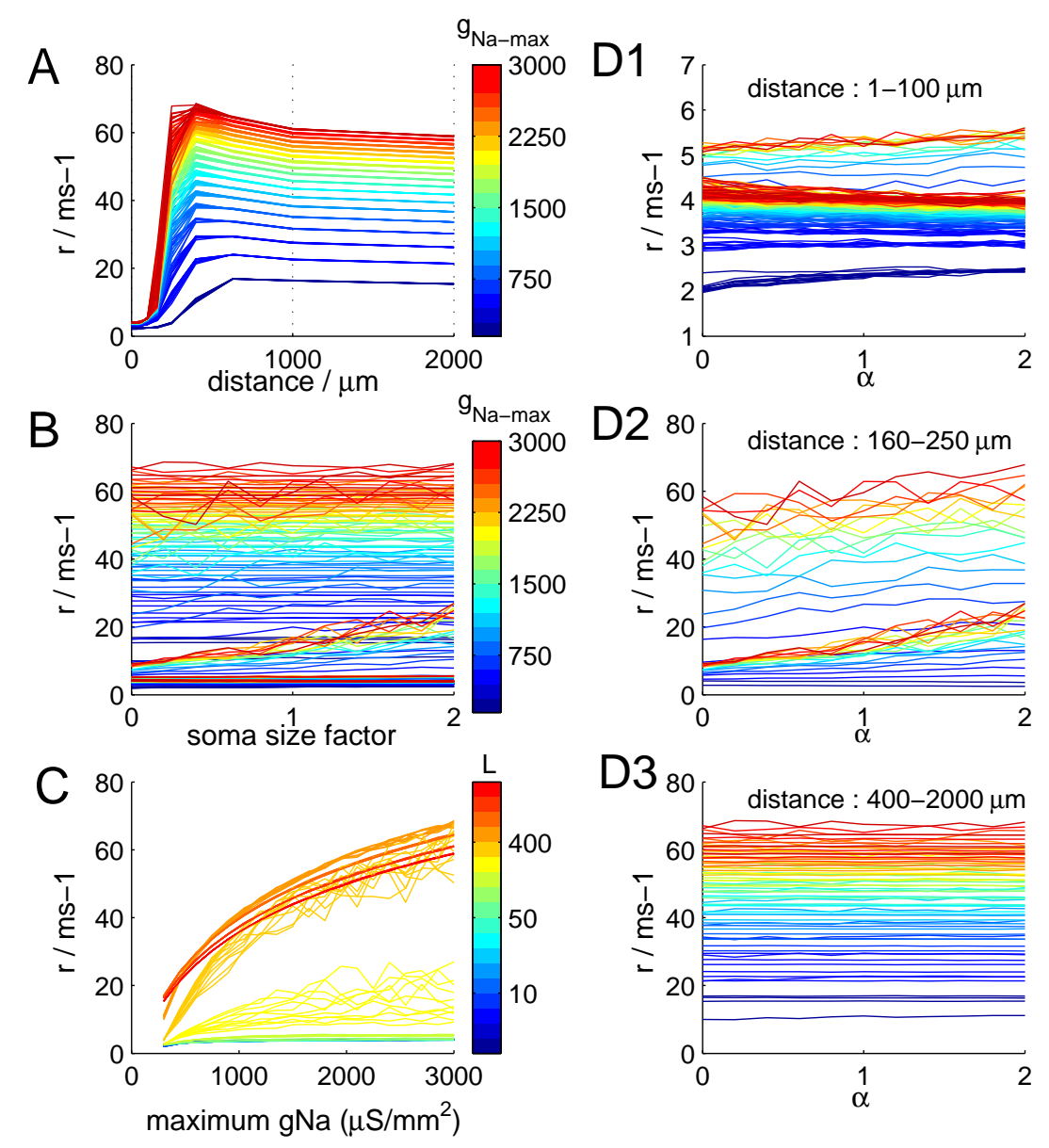

Figure 11.4: Parameter dependence of somatic AP onset rapidness. The onset rapidness of the somatic AP influenced by (A) the distance from initiation site, (B) the soma size factor, and (C) the maximum $\mathrm{Na}^{+}$conductance. (D1-D3) The effects of soma size vary at different ranges of propagation distance. 
saturated at a higher plateau. This resulted from the increase of the MP rate of change with higher channel density. However, when the soma site was close to the AP initiation site $(L<50 \mu \mathrm{m})$, which appearers to be the case in most cortical neurons for orthodromic spikes (Kole et al., 2007; Meeks \& Mennerick, 2007; Palmer \& Stuart, 2006), the onset rapidness was insensitive to the increase of $\mathrm{Na}^{+}$channel density (Figure $11.4 \mathrm{C}$, green traces).

We found different effects of increasing the soma size on the AP waveforms. If the soma was close to the initiation site $(L<50 \mu \mathrm{m})$, the expansion of the soma resulted in a larger current load, hence slowing down the propagating AP (Figure $11.4 \mathrm{D} 1$ ); the effect was neglectable if the soma is far from the initiation site (Figure 11.4 D3); in the transition regime ( $\mathrm{L}$ is between 100 and $250 \mu \mathrm{m}$ ), with high $\mathrm{Na}^{+}$channel density, the somatic current source outweighed the local current load, thus increasing the soma size speeded up AP onset (Figure 11.4 D2).
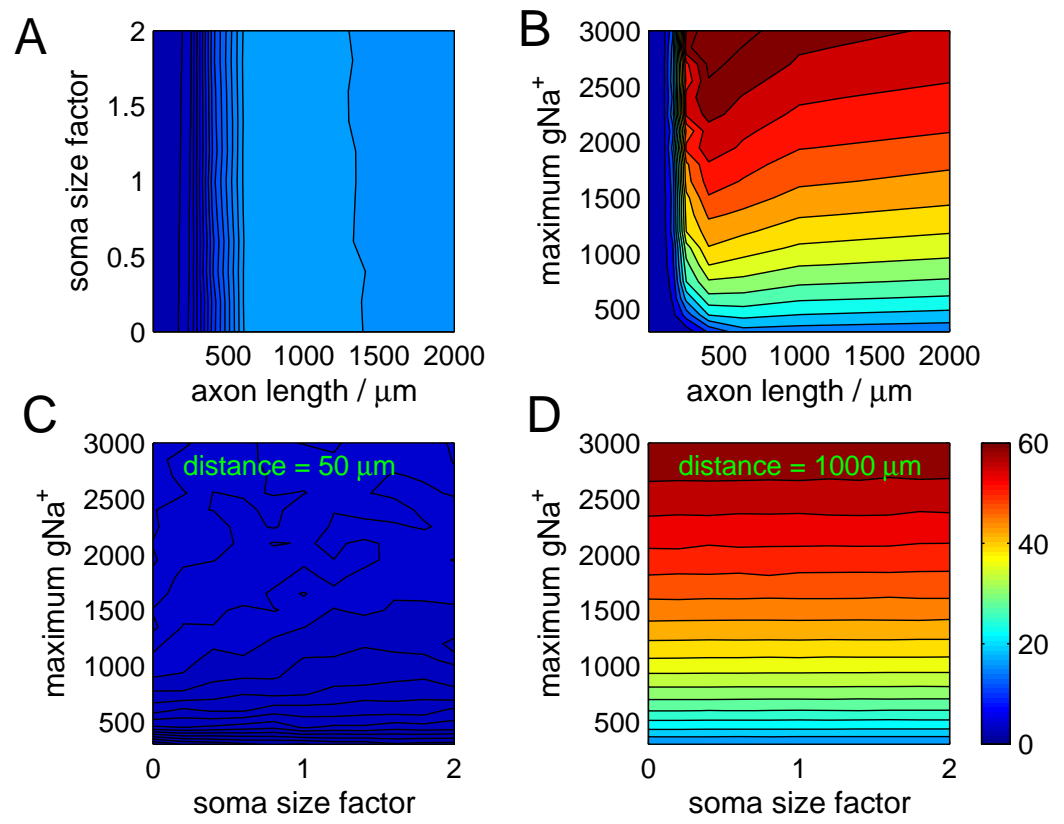

Figure 11.5: Phase diagrams of somatic AP onset rapidness. Parameter regimes with (A) $g_{\mathrm{Na}^{+}}=350 \mathrm{pS} / \mathrm{\mu m}^{2} ;$ (B) $\alpha=1$; (C) $L=50 \mu \mathrm{m}$; (D) $L=1 \mathrm{~mm}$. The color codes for onset rapidness the somatic AP, which is measured by the slope of phase plot at the point of AP onset.

This parameter dependence is illustrated by phase diagrams shown in Figure 11.5. Applying a peak $\mathrm{Na}^{+}$conductance of $350 \mathrm{pS} / \mathrm{\mu m}^{2}$ as in the original Wang-Buzsaki model (Figure 11.5 A), the AP onset rapidness attained the value of $20 \mathrm{~ms}^{-1}$ after propagating for more than one electrotonic length $(\lambda \simeq 400 \mu \mathrm{m})$. Even faster onset can only be induced by higher $\mathrm{Na}^{+}$channel densities. However, regions close to the AP initiation site $(L<100 \mu \mathrm{m})$ were insensitive to an in- 
crease of $\mathrm{Na}^{+}$channel densities (Figure $11.5 \mathrm{~B}$, blue region on the left side). In other words, the rapid onset of the propagating wavefront only occurred in a large distance from the AP initiation site.

\subsection{Onset Rapidness vs. Propagation Velocity}

In this section, we derive an analytical expression for the onset rapidness of the action potential when it propagates far enough to reach the asymptotic state with invariant waveforms.

The cable equation for a homogenous axon can be written as:

$$
\tau \frac{\partial V}{\partial t}=-V+\lambda^{2} \frac{\partial^{2} V}{\partial x^{2}}+i(x, t)
$$

We are searching for the stationary solution of the traveling wave such that the wavefront has an invariant shape, which means, at a given time $t_{1}>t_{0}$, the graph of $V\left(x, t_{1}\right)$ has the same appearance as that of $V\left(x, t_{0}\right)$ except that the wave is moved to the right by the amount of $\theta \cdot t$. The constant $\theta$ represents the propagation velocity of the traveling wave. In order to represent $V(x, t)$ by a function of one variable, we introduce a new variable

$$
y=x-\theta \cdot t,
$$

then Eq. (11.9) becomes

$$
-\tau \theta \frac{\mathrm{d} V}{\mathrm{~d} y}=-V+\lambda^{2} \frac{\mathrm{d}^{2} V}{\mathrm{~d} y^{2}}+i(y)
$$

At the leading edge of the AP, the lateral current is one order of magnitude larger than the local sodium current. We can then neglect the ionic current $i(y)$ and the system is reduced to a linear ordinary differential equation:

$$
V-\tau \theta V^{\prime}-\lambda^{2} V^{\prime \prime}=0
$$

As the upstroke of the action potential approaches the linear phase, we search for a special solution of the form $V=V_{0} \exp (k y)$. The problem then becomes an eigenvalue problem for the equation

$$
1-\tau \theta k-\lambda^{2} k^{2}=0
$$

The positive solution of this equation is

$$
k=\frac{-\tau \theta+\sqrt{\tau^{2} \theta^{2}+4 \lambda^{2}}}{2 \lambda^{2}}
$$


The onset rapidness $r$ is defined as the first derivative of the function $\dot{V}(V)$ at the AP onset point of $\dot{V}=10 \mathrm{~V} / \mathrm{s}$ :

$$
\begin{aligned}
r & =\frac{\partial \frac{\partial V}{\partial t}}{\partial V}=\frac{\partial \frac{\mathrm{d} V}{\mathrm{~d} y} \cdot \frac{\partial y}{\partial t}}{\partial V}=\frac{\partial(k V \cdot \theta)}{\partial V}=k \theta \\
& =-\frac{\tau \theta^{2}}{2 \lambda^{2}}+\frac{\theta \sqrt{\tau^{2} \theta^{2}+4 \lambda^{2}}}{2 \lambda^{2}}
\end{aligned}
$$

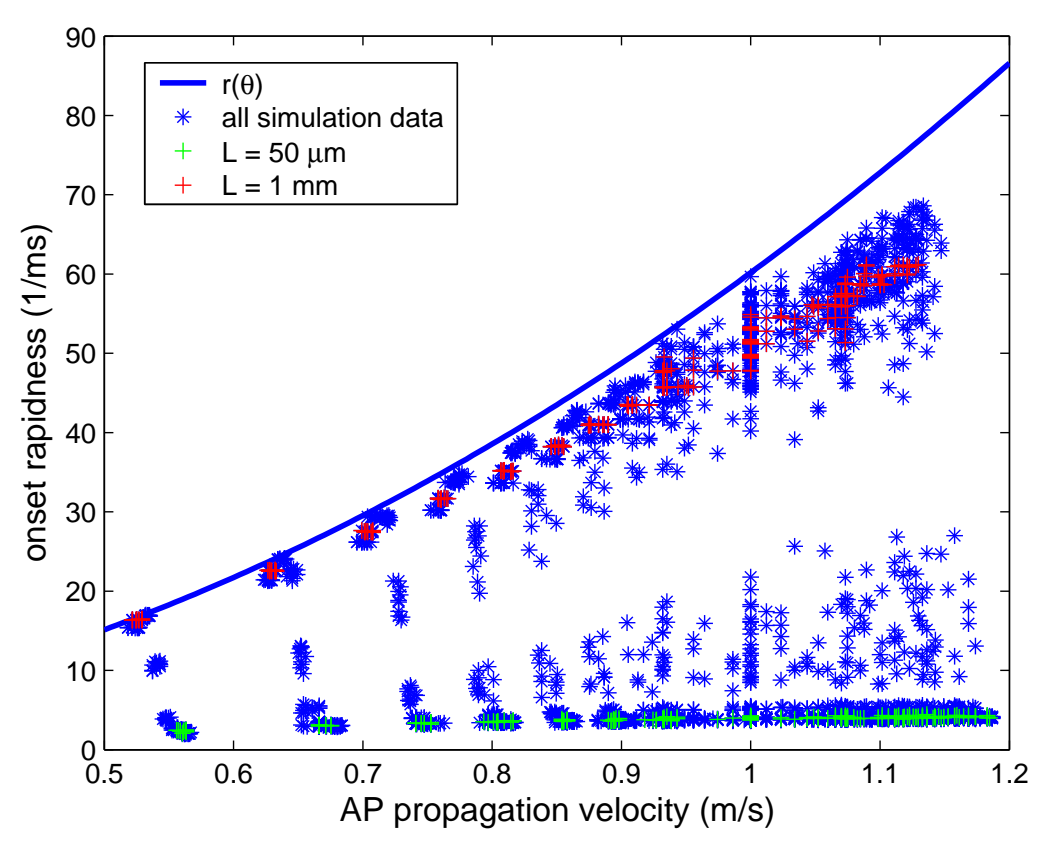

Figure 11.6: AP onset rapidness and the propagation velocity All simulation data from Figure 11.4. The onset rapidness of somatic AP plotted against the propagation velocity of the cable. Blue dots: all data points; red crosses: the subset of data with $\mathrm{L}=1 \mathrm{~mm}$; green crosses: the subset of data with $\mathrm{L}=50$ $\mu \mathrm{m}$; solid line: analytical solution in Eq. 11.16.

To compare the analytical solution with the numerical results, we computed for each simulation the propagation velocity of the traveling waves. The propagation velocity increased with the maximum $\mathrm{Na}^{+}$conductance (data not shown). Figure 11.6 shows the scatter plot of the onset rapidness of the somatic AP versus the propagation velocity recorded at axon. The solid line is the analytical solution of the traveling waves as in Eq. 11.16, which describes the AP onset when the soma is away from the initiation site in the order of several electrotonic length (red crosses: $\mathrm{L}=1 \mathrm{~mm}$ ). This solution gave a good approximation in the regime of low velocity, which means $\bar{g}_{N a}$ is small, thus it was safe to neglect the local ionic current in Eq. (11.9). However, if the distance between soma and initiation 
site was smaller than one electrotonic length (green crosses: $\mathrm{L}=50 \mu \mathrm{m}$ ), the onset rapidness hardly changed with propagation velocity or with increasing $\bar{g}_{N a}$. The data points lie on a horizontal line of the scatter plot and the values of onset speed are much below $10 \mathrm{~ms}^{-1}$.

\subsection{Effects of Lateral and Local Currents}

In the previous section we have examined the asymptotic solution for the traveling wave, where the onset rapidness is mainly determined by the propagation velocity. It raises the question how the AP waveforms are changed by propagation? In particular, how big is the influence of the lateral currents at different propagation distance?
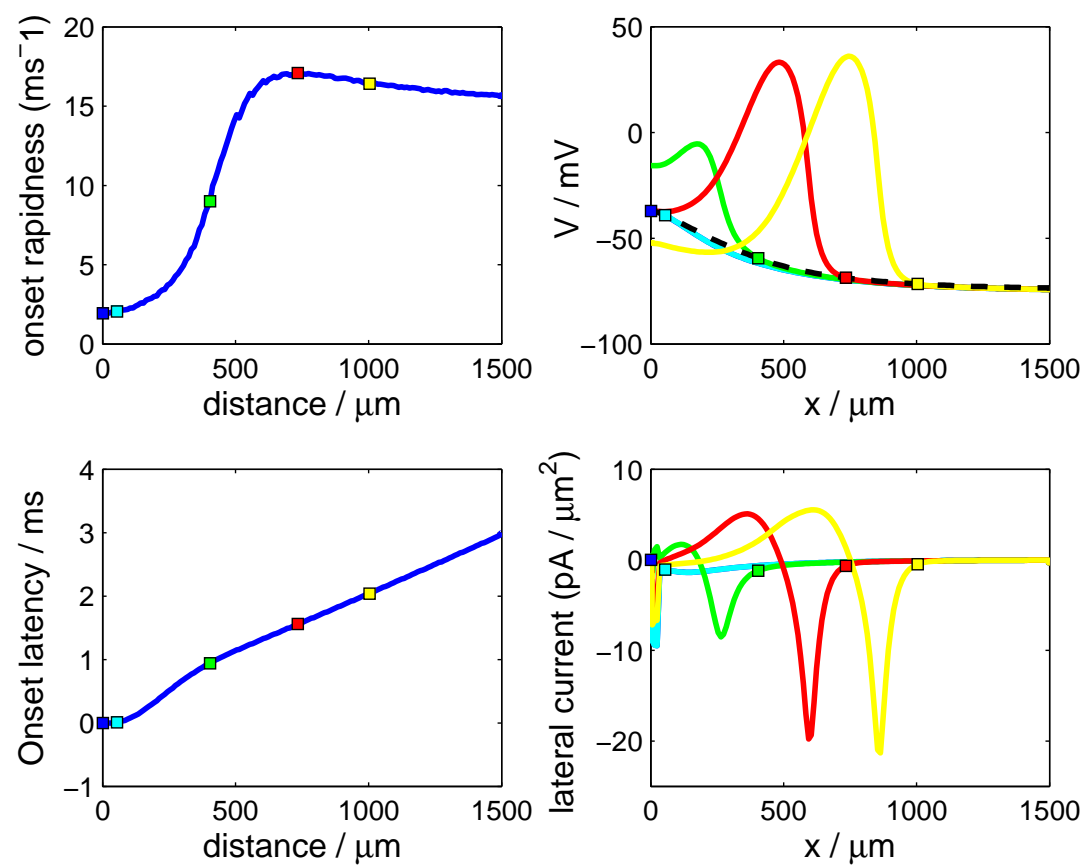

Figure 11.7: The big AP in space and the lateral current. (A) The AP onset rapidness plotted over distance. The blue, cyan, green, red, yellow squares correspond to the distance of 0,50,400,734 and $1000 \mu \mathrm{m}$ from the initiation site. (B) The instantaneous $V(x)$ at the AP onset time of the corresponding locations. The black dashed curve shows the threshold voltage at different locations. (C) The AP onset latency plotted over distance. The squares indicate the same locations with the same colors as in (A). (D) The instantaneous lateral currents at the AP onset time of the corresponding locations. Simulation data from the same example in Figure $11.3 \mathrm{~A}\left(L=50 \mu \mathrm{m}, \alpha=1, g_{\mathrm{Na}}=300 \mathrm{pS} / \mathrm{\mu m}^{2}\right)$. 
To answer this question, we analyzed the spatial potentials and the lateral currents induced by propagation. Figure 11.7] shows the same example as plotted in Figure 11.3. The onset rapidness of the propagating AP gradually increased over a large distance $(L \simeq 2 \lambda)$ and peaked at $L=734 \mu \mathrm{m}$. The onset rapidness underwent a dynamical change even after the propagating velocity saturated to a constant value (Figure 11.7 A, B, green-red-yellow squares). In Figure 11.7 C, we plotted the instantaneous $V(x)$ at the onset time of APs in the four locations marked by squares. Assuming the duration of an AP around $1 \mathrm{~ms}$, and the propagation velocity around $1 \mathrm{~m} / \mathrm{s}$, the spatial AP spreads over a distance as large as $1 \mathrm{~mm}$. However, such a 'big AP' emerged only if it has traveled away from the initiation site for a distance on an order of $\lambda$.

The voltage threshold became more negative while propagating away from the initiation site (Figure 11.7B). At the initiation site, the action potential was generated at the highest voltage threshold, where many $\mathrm{Na}^{+}$channels were inactivated and the peak AP was small. At a distance far from the initiation site AP was mainly triggered by the lateral current. Figure 11.7D plots the lateral currents at the time of $\mathrm{AP}$ onset in the corresponding locations. The instantaneous lateral currents (marked by squares) fed into these sites were similar, indicating the deflection rate of the membrane potential at $10 \mathrm{~V} / \mathrm{s}$ characterized as the onset of spikes. However, the acceleration rate and the peak magnitude of the lateral currents increased significantly by propagating for such distances that a 'big AP' had been established in space.

Besides the local $\mathrm{Na}^{+}$currents and the lateral currents, another current source of the spatial extended neuron came from the constant current injection at the end of the axon. We explored the influence of the injected current by terminating the constant input after the first AP was initiated at the injection site. As shown in Figure 11.8 the following APs were delayed, but the changes of AP waveforms by propagation were not altered by terminating the injected current.

Finally, the increase of onset rapidness by propagation can not simply be explained by the inactivation of the $\mathrm{Na}^{+}$channels induced by the constant current injection. Even if we removed the $\mathrm{Na}^{+}$channel inactivation from the model, the onset dynamics showed the same behavior on the large spatial scale of propagation (data not shown).

\subsection{Decomposing the Somatic AP}

Another experimental evidence that has been invoked to support the lateral current hypothesis are the sometimes observed double peaks on the second derivative of the AP trace. By focal TTX application at the proximal axon one of the peaks can be eliminated, which suggests that orthodromic spikes were initiated in the proximal axon and back propagated to the soma (Meeks \& Mennerick, 2007). A frequently found interpretation is that the first peak of somatic APs results from 

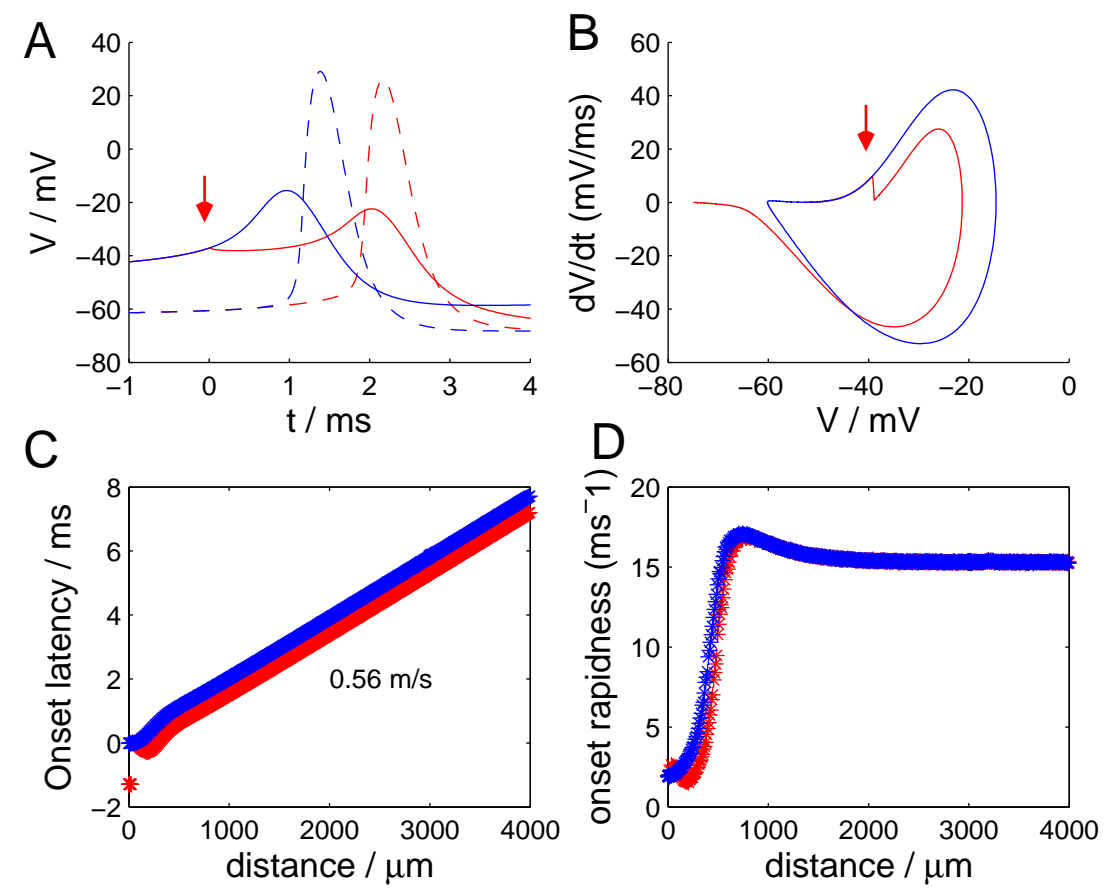

Figure 11.8: AP waveforms affected by termination of the current injection. (A) The AP traces at the current injection site (solid line) and $1 \mathrm{~mm}$ away (dashed line). (B) The phase plots of the APs at the initiation site. (C) Onset latency plotted against the distance. (D) Onset rapidness plotted against the distance. Blue traces: with constant current input; Red traces: the current was terminated at the onset time of the first AP. Arrows: the points of current termination. 
the lateral current invasion from the axon to the soma and the second from the local $\mathrm{Na}^{+}$channel activation at the soma.

In this section we comprehensively characterize the somatic AP waveforms by computing the first and second derivatives of the somatic voltage traces. The traces of $V, \dot{V}, \ddot{V}$ derived from one simulation are shown in Figure 11.9]A. The ratios between the first and the second peaks were computed in each simulation and presented in the phase diagrams shown in Figure 11.9B and Figure 11.9]

A
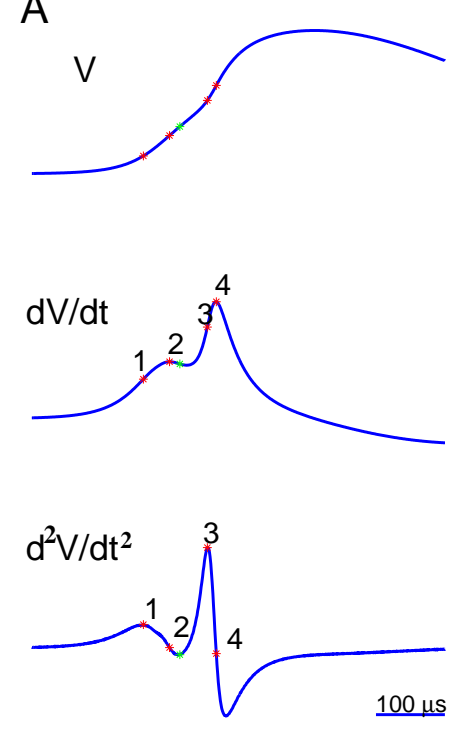
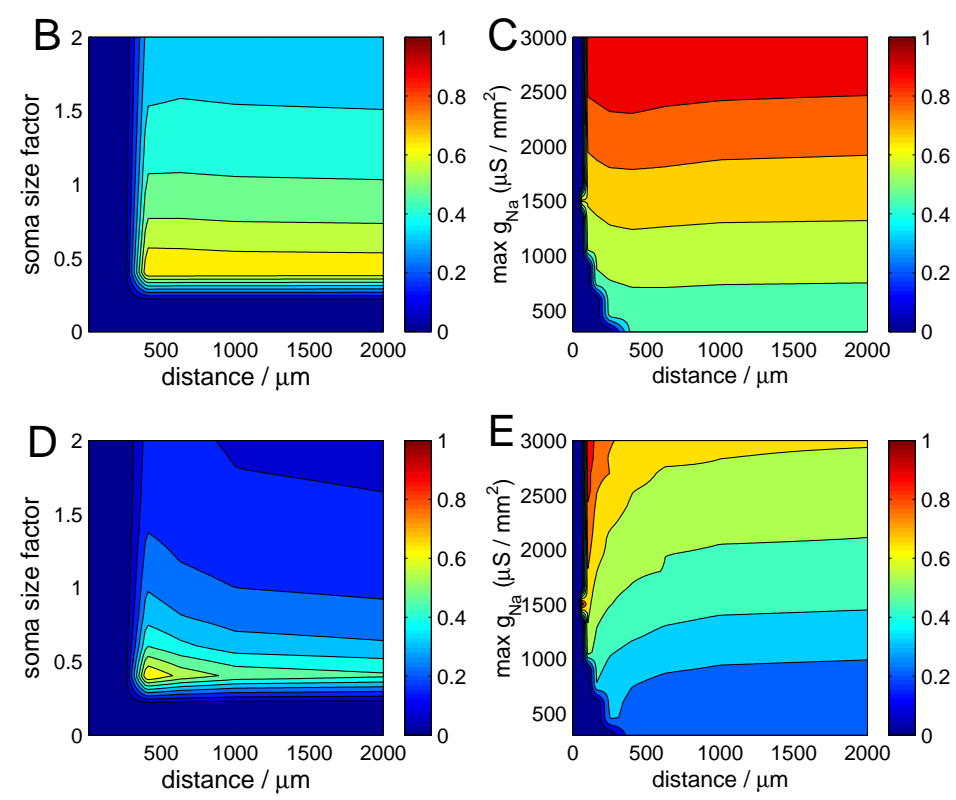

Figure 11.9: Two components of the somatic action potential (A) Example traces of $\mathrm{V}, \dot{V}$, and $\ddot{V} . \mathrm{L}=1000 \mu \mathrm{m}, \alpha=1, \bar{g}_{N a}=300 \mathrm{pS} / \mathrm{\mu m}^{2}$. Peaks of the first and second derivatives are marked by red asterisks. The two peaks of $\ddot{V}$ are numbered by 1 and 3, whereas the two peaks of $\dot{V}$ are numbered by 2 and 4 . (B, C) Color coded representation of the ratios of peak $\dot{V}$ at peak 2 to that at peak 4 in the parameter regimes of (B) $\bar{g}_{N a}=300 \mathrm{pS} / \mu^{2}$ and (C) $\alpha=1$. (D, E) Color coded representation of the ratios of peak $\ddot{V}$ at peak 1 to that at peak 3 in the parameter regimes of (D) $\bar{g}_{N a}=300 \mathrm{pS} / \mu \mathrm{m}^{2}$ and (E) $\alpha=1$.

We first analyzed solutions in which the local $\mathrm{Na}^{+}$channel activation contributed to the major component of the somatic AP, whereas the first peak induced by the lateral current was smaller. From the phase diagram shown in Figure 11.9 B, the somatic AP was monophasic (ratio $\rho=0$ ) if the soma size is smaller than 0.3 or the distance from the initiation site to the soma is shorter than $250 \mu \mathrm{m}$. Enhancing the $\mathrm{Na}^{+}$channel density from $300 \mathrm{pS} / \mathrm{\mu m}^{2}$ to $1200 \mathrm{pS} / \mathrm{\mu m}^{2}$, biphasic APs were found at a distance closer to initiation sites. However, the AP waveforms within the first $50 \mu \mathrm{m}$ of the propagation distance were usually 
A
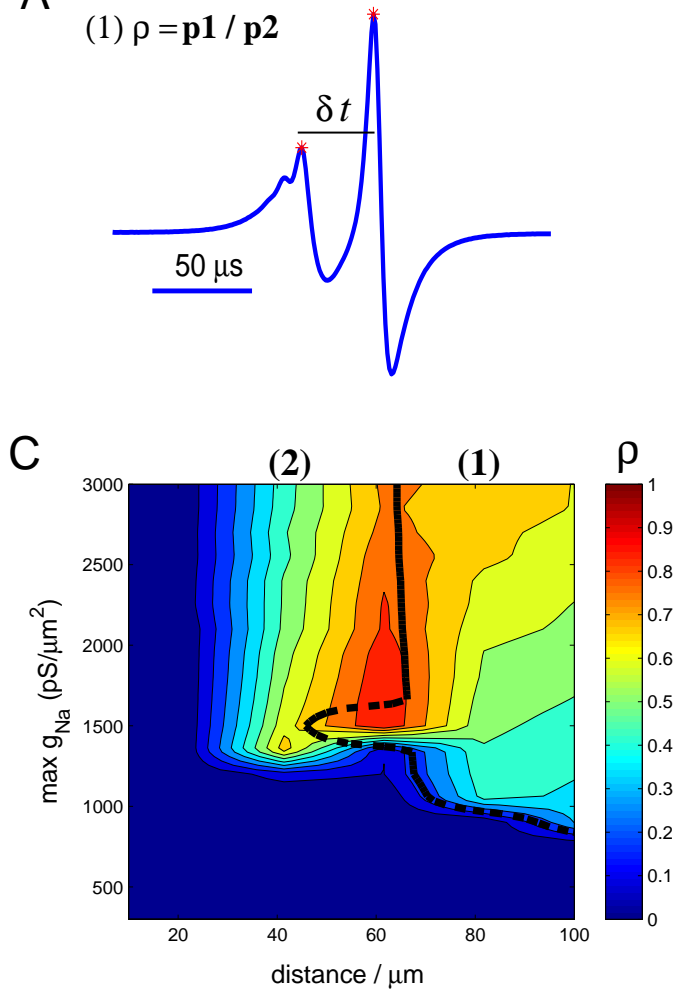

B
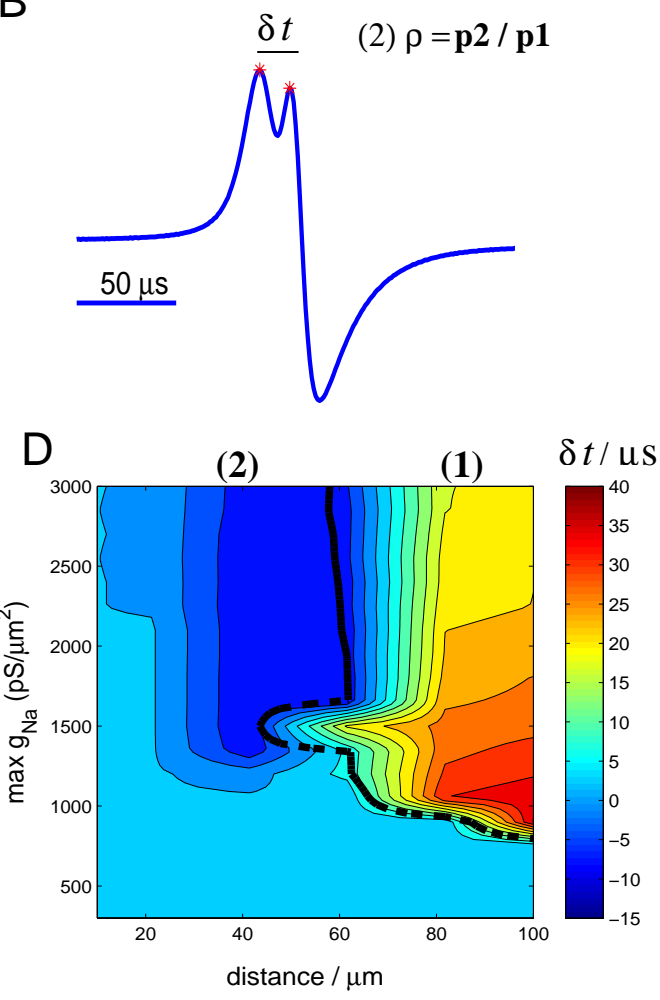

Figure 11.10: Double peaks reveal contributions of the lateral current and the local sodium current on the somatic APs. (A) An example trace of $\ddot{V}\left(\mathrm{~L}=1 \mathrm{~mm}, \alpha=1, \bar{g}_{N a}=1200 \mathrm{pS} / \mathrm{\mu m}^{2}\right)$ shows a smaller peak followed by a larger peak. (B) An example trace of $\ddot{V}\left(L=50 \mu \mathrm{m}, \alpha=1, \bar{g}_{N a}=1200 \mathrm{pS} / \mathrm{\mu m}^{2}\right)$ shows a larger peak preceding a smaller peak. (C) The phase diagram $(\alpha=1)$ of the ratio of the smaller peak value to the larger peak value. (D) The phase diagram $(\alpha=1)$ of the latency between two peaks. Figures are zoomed in to a small range of the distance between $1 \mu \mathrm{m}$ and $100 \mu \mathrm{m}$. Black line shows the boarder line of case (1) and case (2). 
monophasic (blue regions in the phase diagram) even if we further increased the $\mathrm{Na}^{+}$channel density to $3000 \mathrm{pS} / \mathrm{\mu m}^{2}$.

Then we searched for the solutions with a larger 'axonal' peak preceding a smaller 'somatic' peak, which appeared to occur only if the distance from the initiation site to the soma was between 30 and $60 \mu \mathrm{m}$. If they were too close, the smaller peak would merge into the bigger one; if they were too far apart, the traveling wave front arrived before the local $\mathrm{Na}^{+}$channels were activated, hence only the first but not the second peak was left. Such a distance of 30 to 60 $\mu \mathrm{m}$ away from the soma happens to be the location of the AP initiation site as suggested by the experimental recording.

Figure 11.10D depicts the latency between the two peaks. The temporal separation of two peaks indicated the current flow from thin axon to big soma. The latency was shorter $(<20 \mu \mathrm{s})$ when the larger peak of the 'axonal component' proceeded the smaller peak of the 'somatic component'. When a smaller axonal component was followed by a larger 'somatic component', the latency between two peaks was also influenced by the kinetics of local $\mathrm{Na}^{+}$current such that larger $\mathrm{Na}^{+}$channel density results in shorter latency.

In general this short latency does not match the latencies observed in experiments, which might result from one simplification in this study, namely, the homogenous cable property in the geometric model and the instantaneous activation of $\mathrm{Na}^{+}$channels in the Wang-Buzsaki model. Nevertheless, here we present a useful tool to characterize more detailed models with specific properties at AP initiation site.

\subsection{Effects of Delayed Sodium Channel Kinetics}

The Wang-Buzsaki model makes an idealization that the activation of sodium channels is instantaneous. In reality the activation time is finite, although the detailed properties of $\mathrm{Na}^{+}$channel gating and activation kinetics are still to be uncovered for cortical neurons. The activation time constant estimated from in vitro recordings of $\mathrm{Na}^{+}$currents in cortical neurons is around $200 \mu \mathrm{s}$ at $23^{\circ} \mathrm{C}$, and is expected to be even faster at the phvsiological temperature (Baranauskas \& Martina, 2006; Engel \& Jonas, 2005).

To study the impact of delayed channel kinetics we introduced the voltage- and time- dependent activation of sodium channels into our baseline model. Following Hodgkin and Huxley's formalism, the $\mathrm{Na}^{+}$current is controlled by two kinetic processes, activation and inactivation:

$$
I_{N a}=\bar{g}_{N a} m^{n} h\left(V-E_{N a}\right),
$$

where $m$ is the probability that any one of the $n$ independent gating events for activation has occurred, and the exponent $n$ indicates the number of subunit gates. 
A recent study by Baranauskas \& Martina (2006) suggested that the activation time course was better fit by a mono-exponential function rather than the $\mathrm{m}^{3}$ kinetics originally proposed in the Hodgkin-Huxley model. For a systematic comparison of the different candidate models, we therefore constructed two types of channel kinetics, the $\mathrm{WBm}^{1}$ model and the $\mathrm{WBm}^{3}$ model. To facilitate comparison, in both models we use the same activation curve as shown in Figure 11.117 A and the same voltage dependent activation time constant:

$$
\tau_{m}(V)=\varphi /\left(\alpha_{m}(V)+\beta_{m}(V)\right),
$$

where $\varphi$ is a scaling factor to set the maximum activation time constant $\tau_{m}^{0}$ around $200 \mu \mathrm{s}$ (Figure 11.11B, solid line). In both models, if the maximum activation time constant was larger than $200 \mu \mathrm{s}$, slow activation of $\mathrm{Na}^{+}$channels coupled with faster inactivation resulting in bursting of APs (data not shown).

In the $\mathrm{WBm}^{1}$ model the activation variable $m$ approaches its steady state value $m_{\infty}^{3}$ mono-exponentially:

$$
\begin{aligned}
g_{N a} & =\bar{g}_{N a} m h, \\
\tau_{m}(V) \frac{\mathrm{d} m}{\mathrm{~d} t} & =m_{\infty}^{3}(V)-m .
\end{aligned}
$$

And the $\mathrm{WBm}^{3}$ model follows the original $\mathrm{HH}$ model formulation:

$$
\begin{aligned}
g_{N a} & =\bar{g}_{N a} m^{3} h, \\
\tau_{m}(V) \frac{\mathrm{d} m}{\mathrm{~d} t} & =m_{\infty}(V)-m .
\end{aligned}
$$

To depict the different channel kinetics in the $\mathrm{WB}, \mathrm{WBm}^{1}$ and $\mathrm{WBm}^{3}$ models, we applied a voltage step from $-65 \mathrm{mV}$ to $-40 \mathrm{mV}$ and plotted the channel opening probabilities and the corresponding $\mathrm{Na}^{+}$currents in Figure 11.11. As expected from the channel kinetics, the WB model with instantaneous activation exhibited a step increase of the $\mathrm{Na}^{+}$current and then decayed exponentially due to the channel inactivation. In both $\mathrm{WBm}^{1}$ and $\mathrm{WBm}^{3}$ models $\mathrm{Na}^{+}$currents increased as a continuous functions at time initially rising linearly or parabolically $\left(\backsim t^{3}\right)$, respectively.

Figure 11.12A plots spike patterns of these models. Different activation kinetics did not affect the rheobase current to generate action potentials. Given the same level of injected currents, the first AP fired simultaneously in the three models. However, the subsequent APs showed deviation in their time course: APs in the $\mathrm{WBm}^{1}$ model (green trace) preceded those in the $\mathrm{WBm}^{3}$ model, while the WB model exhibited the longest inter-spike intervals. Thus fast activation and inactivation would space the subsequent spikes more sparsely. The phase plots in Figure 11.12B verify that the onset dynamics agrees with the channel kinetics, i.e., fast kinetics resulted in fast onset dynamics. To obtain the same 

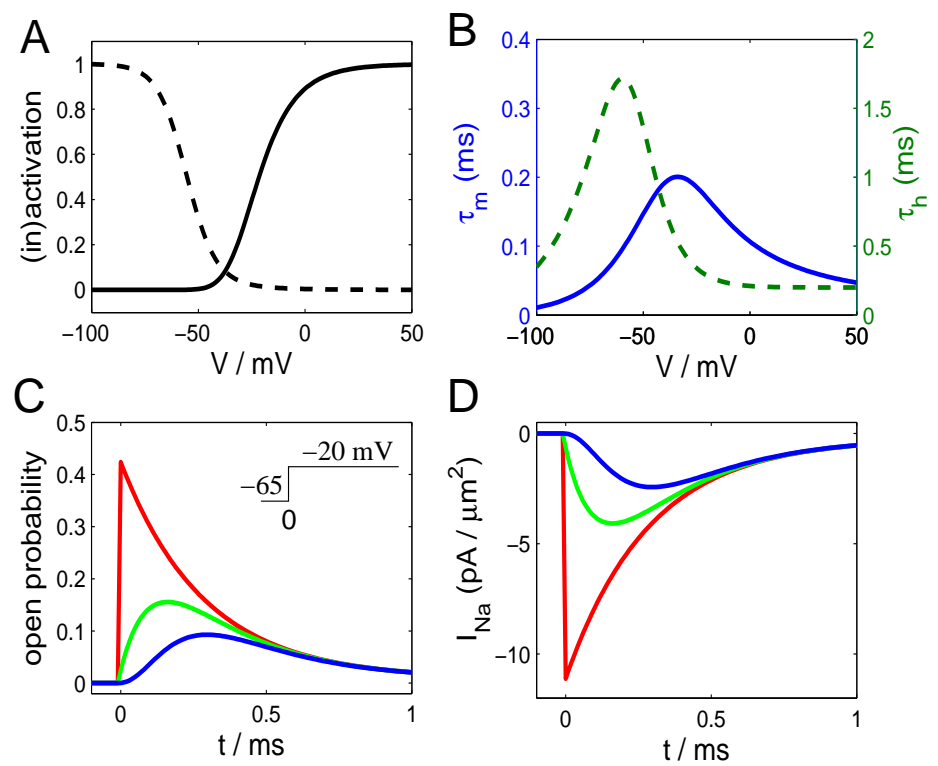

Figure 11.11: $\mathrm{Na}^{+}$Currents in Wang-Buzsaki model with delayed channel kinetics. (A) Activation curve (solid line) and inactivation curve (dashed line). (B) Activation time constant (solid line) and inactivation time constant (dashed line). (C) The channel open probabilities change with a step of the command voltage from -65 to $-40 \mathrm{mV}$ at $\mathrm{t}=0$. (D) The $\mathrm{Na}^{+}$currents under the same voltage clamp protocol. red: WB model; green: $\mathrm{WBm}^{1}$ model; blue: $\mathrm{WBm}^{3}$ model. 

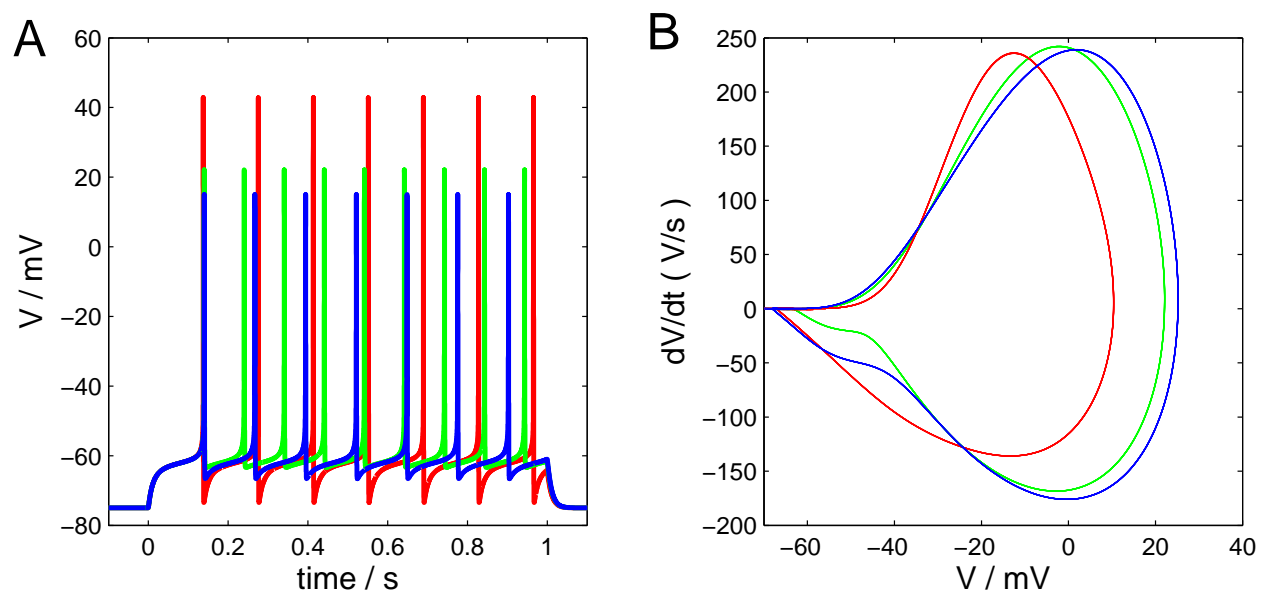

Figure 11.12: Single compartment WB Models with $m^{1}$ and $m^{3}$ kinetics. Red trace: WB model; Green trace: $\mathrm{WBm}^{1}$ model; Blue trace: $\mathrm{WBm}^{3}$ model. (A) The spike patterns generated by the same constant current input: Ie = $10 \mathrm{nA} / \mathrm{mm}^{2}, \overline{\mathrm{g}}_{\mathrm{Na}}=600 \mathrm{pS} / \mathrm{\mu m}^{2}$. (B)Phase plots explicit onset dynamics of the three models. The maximum $\mathrm{Na}^{+}$channel conductances were adjusted such as to obtain similar levels of peak $\mathrm{d} V / \mathrm{d} t$ values. WB model: $\overline{\mathrm{g}}_{\mathrm{Na}}=300 \mathrm{pS} / \mathrm{\mu m}^{2}$; $\mathrm{WBm}^{1}$ model: $\overline{\mathrm{g}}_{\mathrm{Na}}=600 \mathrm{pS} / \mathrm{\mu m}^{2} ; \mathrm{WBm}^{3}$ model: $\overline{\mathrm{g}}_{\mathrm{Na}}=750 \mathrm{pS} / \mathrm{\mu m}^{2}$.

level of peak $\dot{V}$, a higher maximum $\mathrm{Na}^{+}$channel conductance was required in compensate of the slower activation kinetics.

Then we applied the different kinetic models to the multi-compartment simulations of the geometric neuron. The AP onset rapidness was quantified for different combinations of the three parameters $L, \alpha$, and $\bar{g}_{N a}$ using the same procedure as described before for the WB model. The simulation results are shown in Figure 11.13. The parameter dependencies of the AP onset dynamics were similar as that in the WB model except that the peak onset rapidness was dramatically reduced from $70 \mathrm{~ms}^{-1}$ (WB model) to $20 \mathrm{~ms}^{-1}$ (WBm ${ }^{1}$ model) and $10 \mathrm{~ms}^{-1}$ (WBm ${ }^{3}$ model $)$.

Another Hodgkin-Huxley type $m^{3}$ model widely used in cortical neuron simulations is the Mainen-Sejnowski model (Mainen et al., 1995). More details about its channel kinetics can be found in the appendix to Chapter 9. Figure 11.14 summarizes the onset rapidness of somatic APs obtained by applying MainenSejnowski channel kinetics in the geometric model. The effect induced by propagation was qualitatively the same but quantitatively much smaller in this model, with the maximum value of onset rapidness below $10 \mathrm{~ms}^{-1}$ in all simulations. 

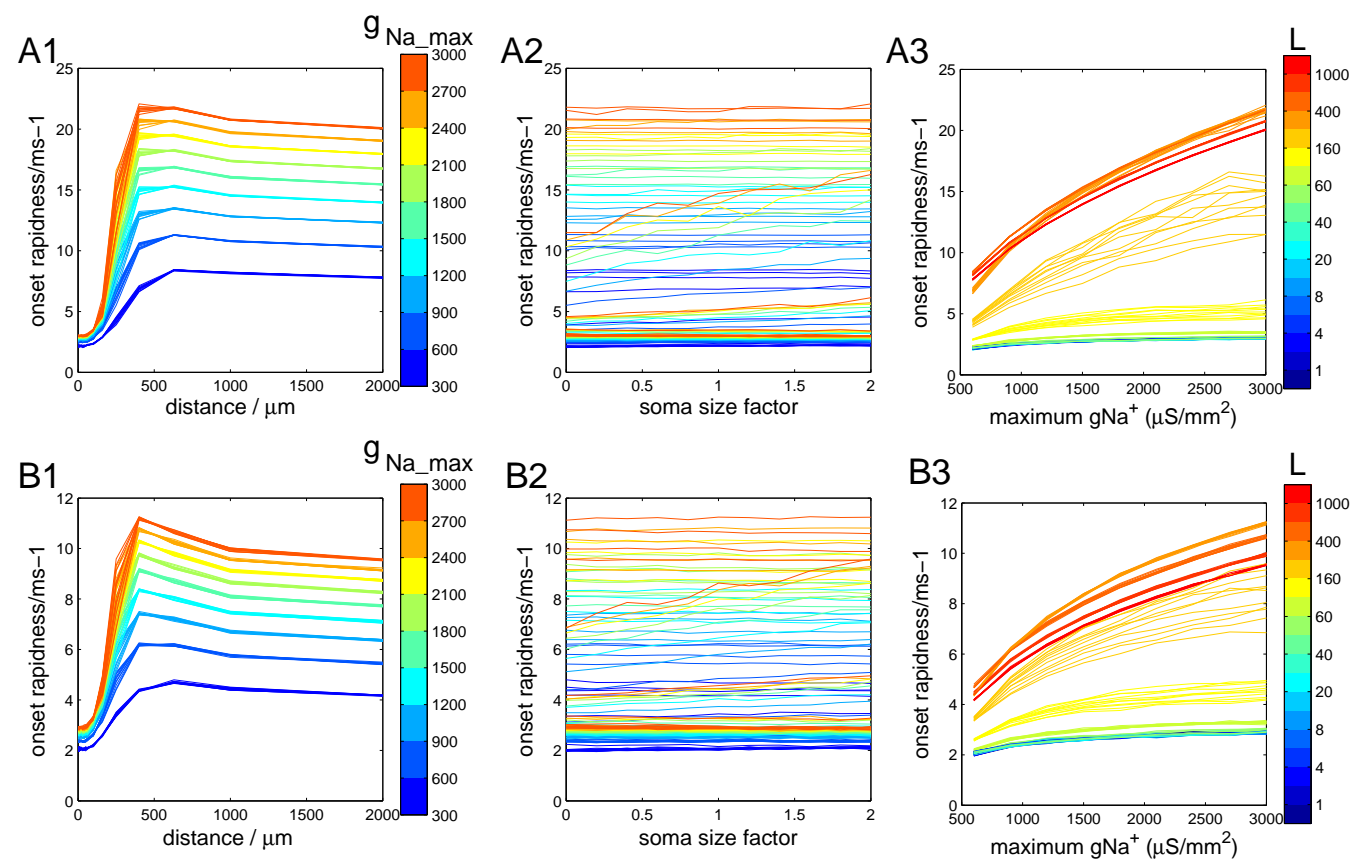

Figure 11.13: Somatic AP onset in $\mathbf{W B} \mathbf{m}^{\mathbf{1}}(\mathbf{A})$ and $\mathbf{m}^{\mathbf{3}}(\mathrm{B})$ models The onset rapidness of somatic AP depends on (A1, B1) the distance from initiation site, (A2, B2) the soma size factor and (A3, B3) the maximum $\mathrm{Na}^{+}$conductance. 

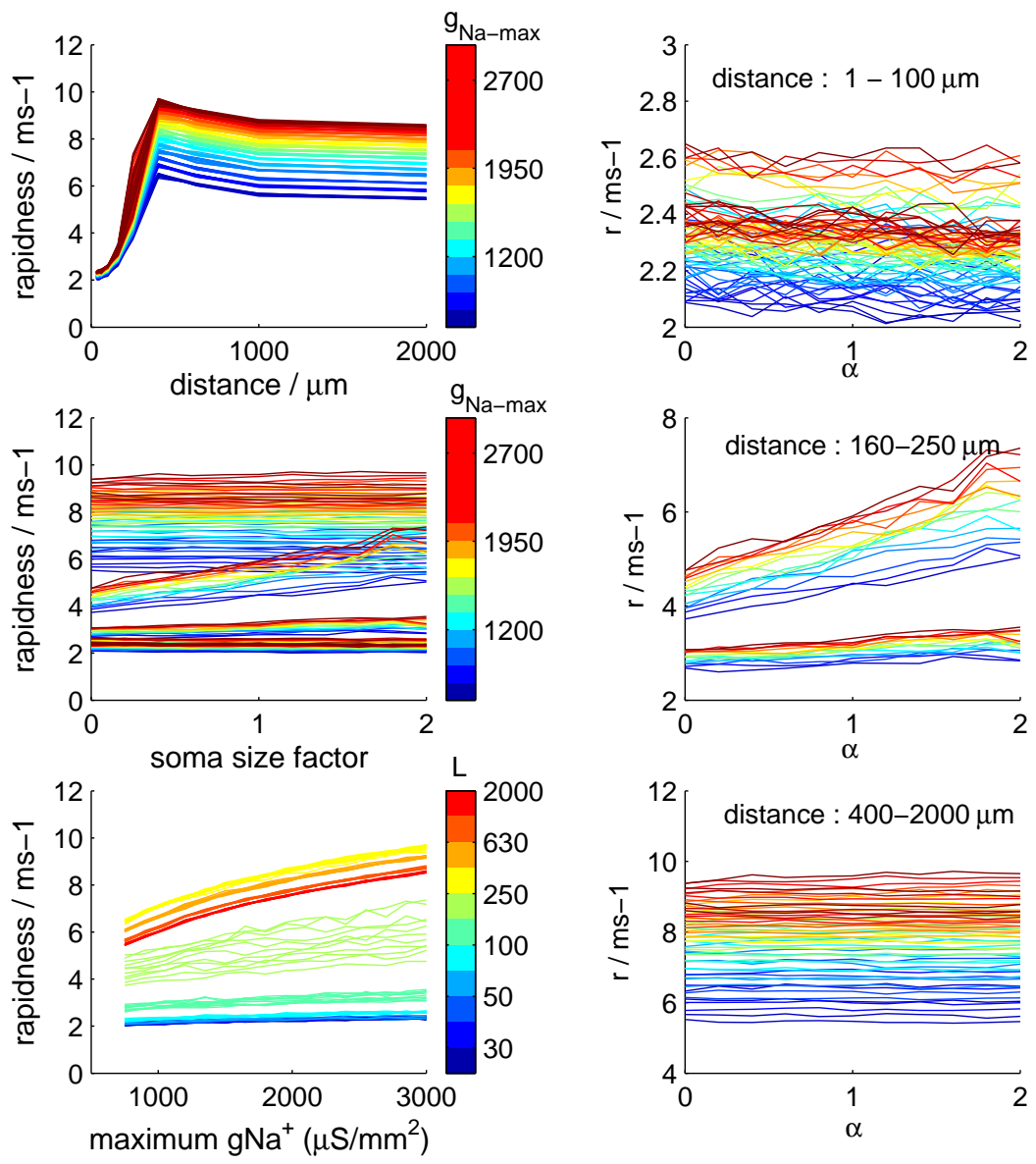

Figure 11.14: Somatic AP onset in the Mainen-Sejnowski model The onset rapidness of the somatic AP depends on (A) the distance from initiation site, (B) the soma size factor, and (C) the maximum $\mathrm{Na}^{+}$conductance. (D1-D3)The soma size effects vary at different distance ranges. 


\subsection{Summary and Discussion}

In this chapter we presented a comprehensive study of the somatic action potential waveforms using an idealized geometric model with axonal AP initiation and back propagation.

First, the waveforms of the somatic APs were quantified by the measure of onset rapidness. Its parameter dependence was characterized with respect to the distance from the AP initiation site to the soma, the soma size, and the overall $\mathrm{Na}^{+}$channel density.

In our simulations the waveforms of APs exhibited a transition from an initially slow onset to a rapid onset with increasing propagation distance. If the spikes were triggered within $50 \mu \mathrm{m}$ away from the soma, the onset dynamics of somatic APs was as slow as that at the initiation site. At a large propagation distance, the traveling APs reached an invariant waveform. For these traveling APs, we derived the onset speed as a function of the propagation velocity (Eq. 11.16).

The impact of soma geometry was found to depend on the propagation distance. If the propagation distance was small $(L \ll \lambda)$, expansion of the soma area introduced a larger current load, so that the AP onset was slowed down; If the propagation distance was large $(L>\lambda)$, the traveling wave had reached an invariant waveform such that increasing the soma size had hardly any impact on the onset dynamics of the somatic AP; At an intermediate distance in the transition regime $(L \approx \lambda)$ with a high $\mathrm{Na}^{+}$channel density, the local current source outweighed the current load, thus a larger somatic membrane area actually speeded up the AP onset in the middle point of the soma.

$\mathrm{Na}^{+}$currents provide the major current source for the generation of APs. A higher $\mathrm{Na}^{+}$channel density was reflected by increase of the peak rate of rise $\mathrm{d} V / \mathrm{d} t$. The impact on the AP onset dynamics differed at different propagation distance. Close to the initiation site, the onset dynamics remained slow and was rather insensitive to an increase in $\mathrm{Na}^{+}$channel density. At an intermediate propagation distance, increasing $\mathrm{Na}^{+}$channel density dramatically increased the onset speed; At a large distance, increasing $\mathrm{Na}^{+}$channel density speeded up the propagation velocity, thus the onset speed was increased correspondingly.

Second, we investigated the relative impacts of the lateral and local currents. Our results suggested that APs propagating over a distance on the order of one electrotonic length established a strong potential field in space, generating a large lateral current. The contributions of lateral currents and local $\mathrm{Na}^{+}$currents to the somatic AP waveforms were revealed by the double peaks on the traces of $\mathrm{d} V / \mathrm{d} t$ and $\mathrm{d}^{2} V / \mathrm{d} t^{2}$. Two components in the first and second derivatives could be detected only if the soma size, the distance, and the channel density were within a certain range. Particularly for a larger lateral current to preced a smaller local $\mathrm{Na}^{+}$current, this could happen only within a small range of the propagation distance between 30 and $60 \mu \mathrm{m}$. The corresponding somatic APs exhibit slow onset regardless of the double peaks in $\ddot{V}$. Thus biphasic APs, even with a salient 
peak induced by invasion of axonal APs, do not necessarily result in fast onset.

Finally, different models of Hodgkin-Huxley type show qualitatively the same parameter dependence of the AP onset dynamics. The changes induced by propagation were more prominent with faster $\mathrm{Na}^{+}$channel activation kinetics, i.e., the onset dynamics of somatic APs was fastest in the WB model, slower in the $\mathrm{WBm}^{1}$ and $\mathrm{WBm}^{3}$ model, and even slower in the Mainen-Sejnowski models. 


\section{Chapter 12}

\section{Dynamics of AP Initiation in the Soma-Proximal Axon-Complex}

\section{$12.1 \quad$ Introduction}

In view of the comprehensive study of the simplified geometric model in Chapter 11. one might argue that the spatial inhomogeneity of electrical properties in a real neuron could shape significantly the AP initiation and propagation. In the following, we characterize the impact of AP initiation and propagation on somatic AP waveforms using a myelinated axon while implementing the channel kinetics as realistic as possible. To distinguish it from the simplified geometric model we term it 'the full model'.

The myelinated axon is not a homogeneous cable but can be subdivided into functionally different regions of axon hillock, axon initial segment(AIS), the internode myelinated segment, and the nodes of Ranvier.

Though the soma is considered the integration site of incoming synaptic inputs, it is not the spike initiation site. Axonal initiation of action potential has long been demonstrated in cortical neurons (Stuart et al., 1997). Voltage sensitive dye imaging (VSDI) studies have suggested that AP initiate at AIS of pyramidal neurons, about 20-40 $\mathrm{m}$ away from the axon hillock (Palmer \& Stuart, 2006), which was corroborated by simultaneous somatic and axonal recordings (Yu et al., 2008).

The region of AIS is characterized by intense antibody labeling of voltagegated $\mathrm{Na}^{+}$channels and cytoskeleton proteins. The exact location of the peak channel density has however remained ambiguous. Peak channel densities have been found at $10 \mu \mathrm{m}$ (Kole et al., 2008) or 40-50 $\mu \mathrm{m}$ (Meeks \& Mennerick, 2007) from the soma, or in the first $50 \mu \mathrm{m}$ of the proximal axon (Hedstrom \& Rasband, 2006; Inda et al., 2006). Neither soma nor proximal dendrite was visible by $\mathrm{Na}^{+}$channel antibody staining.

Assuming that channels have similar ion gating properties, a surplus of chan- 
nels in the AIS should lead to significant larger $\mathrm{Na}^{+}$current at AIS. However electrophysiological studies using either cell-attached or outside-out patch clamp mode revealed only a small difference between soma, proximal dendrite and AIS, incompatible with the large density differences (Colbert \& Pan, 2002; Kole et al., 2008).

In this chapter we constructed a full model of myelinated neuron according to experimental observations. By varying the length of the AIS alongside its $\mathrm{Na}^{+}$channel density in simulations, we tested the resulting AP initiation sites and the corresponding waveforms of somatic spikes. In particular, neurons with passive dendrites showed different behaviors compared to neurons with uniform $\mathrm{Na}^{+}$channel distributions across soma and dendrite.

\subsection{Model Description}

\subsubsection{Model Neuron Morphology}

The morphology of the full model (Figure 12.1) was contructed according to $\mathrm{Na}^{+}$imaging recordings of pyramidal neurons (Data provided by Michael Gutnick, Hebrew University).

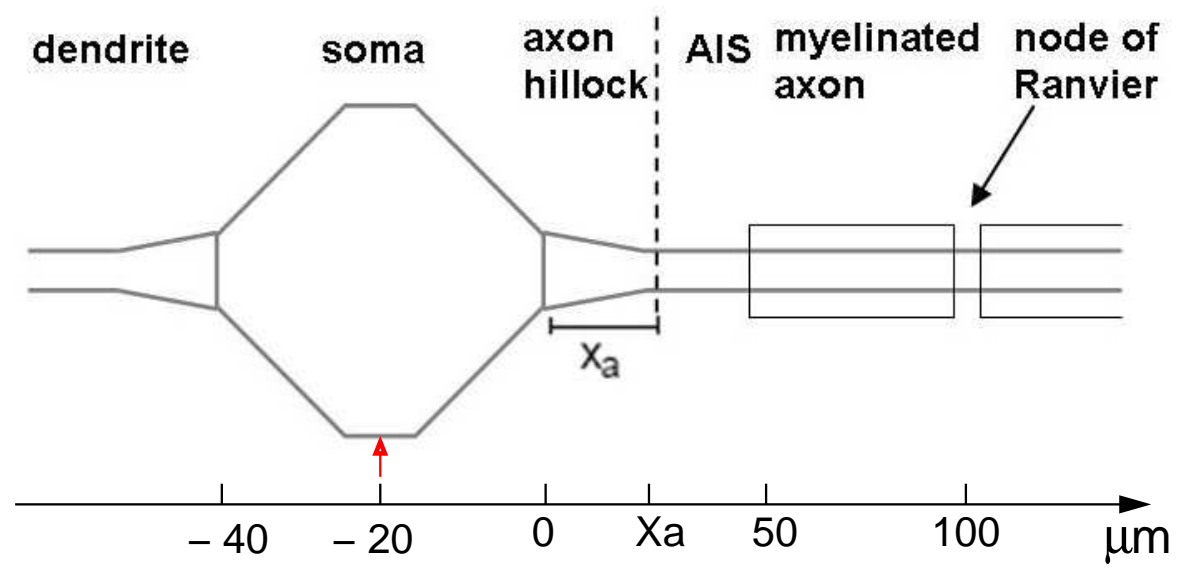

Figure 12.1: Modeling the morphology of a myelinated cortical neuron Details refer to the text. Distance is measured from the end of the soma to the axon. Red arrow indicates current injection into the middle of the soma.

The soma was modeled as an octagon with a length of $40 \mu \mathrm{m}$ and a maximum width of $20 \mu \mathrm{m}$. The total somatic membrane area is $1414 \mathrm{\mu m}^{2}$, which was divided to 20 segments of $2 \mu \mathrm{m}$ length.

Attached to the soma is a myelinated axon consisting of axon hillock $\left(x_{a}\right.$ in length, tapping from $3 \mu \mathrm{m}$ to $1 \mu \mathrm{m}$ in diameter), the axonal initial segment (AIS, 
$\left(50-x_{a}\right) \mu \mathrm{m}$ in length, $1 \mu \mathrm{m}$ in diameter), the alternating myelinated internodes (50 $\mathrm{mm}$ in length, $1 \mu \mathrm{m}$ in diameter, 25 segments) and nodes of Ranvier (2 $\mu \mathrm{m}$ in length, $1 \mu \mathrm{m}$ in diameter, one segment).

At the other end of the soma is the dendrite, which was modeled as an equivalent cylinder tapping from $2 \mu \mathrm{m}$ at the proximal part to $1 \mu \mathrm{m}$ at the distal part. The proximal dendrite of the first $100 \mathrm{\mu m}$ was divided to 10 segments of $10 \mu \mathrm{m}$ segment length, and the distal dendrite in length of $500 \mu \mathrm{m}$ was divided to 7 segments of $50 \mathrm{\mu m}$ segment length in Section 12.3. The passive dendrite in Section 12.4 consists of 58 segments of distal dendrite, yielding a total dendritic length of $3000 \mu \mathrm{m}$ and a total membrane area of $9582 \mathrm{\mu m}^{2}$.

\subsubsection{Passive Electrical Parameters}

Passive parameters were adjusted to match experimental observations. Membrane capacitance $C_{\mathrm{m}}$ was assumed to be $10 \mathrm{nF} / \mathrm{mm}^{2}$ everywhere except that for the myelinated part, $C_{\mathrm{m}}^{\text {myelin }}=0.1 \mathrm{nF} / \mathrm{mm}^{2}$. The leak conductance of somatic membrane was taken as $g_{\mathrm{L}}=1 \mathrm{pS} / \mathrm{\mu m}^{2}$ according to the Wang-Buzsaki model. Thus the somatic region has a membrane resistance (the inverse of the leak conductance) of $1 \mathrm{M} \Omega \cdot \mathrm{mm}^{2}$ and a membrane time constant of $10 \mathrm{~ms}$. Due to myelination the membrane of internode parts is supposed to be less leaky than that of nodes. We chose the leak conductance of the nodes and the internode segements $g_{\mathrm{L}}^{\text {node }}=10 \mathrm{pS} / \mathrm{\mu m}^{2}$ and $g_{\mathrm{L}}^{\text {myelin }}=0.1 \mathrm{pS} / \mathrm{\mu m}^{2}$, correspondingly. The intracelluar resistivity $r_{L}$ was assumed to be a constant value of $5 \mathrm{k} \Omega \cdot \mathrm{mm}$ across the neuron, which is an important quantity determining the electrotonic length and the input resistance of the neuron, as discussed later.

Simulations were performed to test the sensitivity of the model behavior to different choices of passive parameters. In particular, our baseline model was set to fullfill three requirements:

- The axonal electrotonic length $\lambda \simeq 400 \mu \mathrm{m}$ (Shu et al., 2006).

- Onset latency between soma and initiation site $\Delta t \simeq 100 \mu$ s (Stuart et al., 1997; Clark et al., 2005; Palmer \& Stuart, 2006; Kole et al., 2007).

- AP propagating speed $\theta \simeq 1 \mathrm{~m} / \mathrm{s}$ (Stuart et al., 1997; Kole et al., 2007).

\section{Electrotonic Length}

The linear cable equation for mathematical analysis of signal propagation down a cable-like structures of axons or dendrites is written as:

$$
\tau_{M} \frac{\partial V}{\partial t}=\lambda^{2} \frac{\partial^{2} V}{\partial x^{2}}-V+r_{m} i_{e},
$$




\section{Dynamics of AP Initiation in the Soma-Proximal Axon-Complex}

where $\tau_{M}$ and $\lambda$ measure the temporal and spatial scales of the membrane potential dynamics. For a cable of radius $a$, membrane resistence $r_{m}$, and intracellular resistivity $r_{L}, \lambda$ is given by

$$
\lambda=\sqrt{\frac{a r_{m}}{2 r_{L}}} .
$$

Thus, the steady state membrane potential along a cable for a constant current injection at the point $x=0$ is described as:

$$
V(x)=I_{e} R_{\text {in }} \exp \left(-\frac{|x|}{\lambda}\right),
$$

where $I_{e}$ is the total amount of injected current, and $R_{\text {in }}$ is the input resistance at injection site.

Since the axon consists of alternating myelinated internodes ( $50 \mu \mathrm{m}$ in length, $1 \mu \mathrm{m}$ in diameter) and nodes of Ranvier ( $2 \mu \mathrm{m}$ in length,1 $\mu \mathrm{m}$ in diameter), the 'hybrid' electrotonic length $\lambda$ is obtained by solving the equation:

$$
\frac{x_{1}+x_{2}}{\lambda}=\frac{x_{1}}{\lambda_{1}}+\frac{x_{2}}{\lambda_{2}}
$$

For the myelinated axon, $x_{1}=50 \mu \mathrm{m}, x_{2}=2 \mu \mathrm{m}, a=0.5 \mu \mathrm{m}$. We assumed an invariant intracelluar resistivity $r_{L}=5 \mathrm{k} \Omega \cdot \mathrm{mm}$ so that $\lambda_{1}=500 \mu \mathrm{m}$ and $\lambda_{2}=50 \mu \mathrm{m}$ from Eq12.2, yielding $\lambda=371 \mu \mathrm{m}$.

\subsubsection{Channel Distribution}

The channel kinetics of the Wang-Buzsaki model was summarized in the appendix of Chapter 9. The maximum $\mathrm{Na}^{+}$and $\mathrm{K}^{+}$conductances at soma were taken as $300 \mathrm{pS} / \mathrm{\mu m}^{2}$ and $150 \mathrm{pS} / \mathrm{\mu m}^{2}$, respectively.

Assuming a similarity between soma and axon hillock, we used the same channel density at axon hillock as the somatic density. The maximum $\mathrm{Na}^{+}$conductance $\bar{g}_{\mathrm{Na}}$ at the axon initial segment (AIS) was varied between $300 \mathrm{pS} / \mathrm{\mu m}^{2}$ and $6000 \mathrm{pS} / \mathrm{\mu m}^{2}$ so as to explore its impact on the AP initiation sites and the somatic AP waveforms.

The $\mathrm{Na}^{+}$channel density in the nodes of Ranvier was assumed to be $3000 \mathrm{pS} / \mathrm{um}^{2}$, 10-fold of the somatic density. Additional simulations were performed at a higher nodal $\mathrm{Na}^{+}$channel density of up to 50 -fold, $15000 \mathrm{pS} / \mathrm{um}^{2}$, which was found not to alter the results presented below. For simplicity the active components of voltage-gating channels were assumed to be absent in the myelinated segments.

Physiological studies have revealed similar $\mathrm{Na}^{+}$channel densities between soma and apical dendrites in pyramidal neurons by either whole cell (Huguenard et al., 1989) or single channel patch recordings (Huguenard et al. 1989: Stuart \& Sakmann, 1994; Kole et al., 2008). In Section 12.3 we applied a uniform $\mathrm{Na}^{+}$channel 
distribution throughout soma and dendrite. As a comparison, we used a passive dendrite with only $10 \%$ of somatic channel density in Section 12.4.

The $\mathrm{K}^{+}$channel density was adjusted from the baseline level of somatic $\bar{g}_{\mathrm{K}}$ proportionally to the $\mathrm{Na}^{+}$channel density, so that the basic shape of AP waveforms was maintained while varying the channel density.

\subsection{Models with Active Dendrites}

In simulations of models with active dendrites we systematically varied the starting point $x_{a}$ of AIS ranging between $5 \mu \mathrm{m}$ and $45 \mu \mathrm{m}$ measured from the end of soma. The $\mathrm{Na}^{+}$channel density $\bar{g}_{\mathrm{Na}}$ at AIS varied from $300 \mathrm{pS} / \mathrm{\mu m}^{2}$ to $3900 \mathrm{pS} / \mathrm{\mu m}^{2}$. A constant current was injected into the soma and the level of current was adjusted such that the model neurons fire repetitively at $10 \mathrm{~Hz}$.

Figure 12.2 shows an example with $x_{a}=20 \mu \mathrm{m}$ and $\bar{g}_{\mathrm{Na}}=900 \mathrm{pS} / \mathrm{\mu m}^{2}$. The local membrane potentials were recorded simutaneously thoughout the neuron (Figure 12.2A). The onset of action potential was detected when the rate of change $\mathrm{d} V / \mathrm{d} t$ crossed $10 \mathrm{~V} / \mathrm{s}$. The AP initiation site was recognized at the location with the most negative onset latency compared to the somatic AP onset (Figure 12.2C). In this typical example, an AP was initiated at the axonal site of $35 \mu \mathrm{m}$ away from the end of the soma with an onset latency of around -100 $\mu \mathrm{s}$.

The onset rapidness of APs was measured by the slope on the phase plot (Figure 12.2B) at the point of AP onset, which sustained a value as low as $3 \mathrm{~ms}^{-1}$ in both the initiation site and the soma. The onset rapidness started to increase only after a distance of $100 \mu \mathrm{m}$ propagating away from soma (Figure 12.2D).

We compared these simulation results with those of the simplified 'ball-andstick' models in the previous chapter (Figure 11.3). The models behaved qualitatively similar, particularly with respect to the fast onset requiring a minimum propagation distance of $\sim 100 \mu \mathrm{m}$.

\subsubsection{Sites of AP Initiation}

In the full model of a myelinated neuron, we applied the current injection at the soma and searched for parameter regimes with action potentials preferentially initiated at the axonal initial segement (AIS). In this model, the axonal APs were triggered by applying a higher $\mathrm{Na}^{+}$channel density $\bar{g}_{\mathrm{Na}}$ at AIS compared to that at the soma.

We recorded the sites of AP initiation in different simulations varying the starting point $x_{a}$ of the AIS and the maximum $\mathrm{Na}^{+}$channel conductance $\bar{g}_{\mathrm{Na}}$ at AIS (Figure 12.3). In these models, a channel density of $\bar{g}_{\mathrm{Na}}>900 \mathrm{pS} / \mathrm{\mu m}^{2}$ was sufficient to bring the AP initiation site from the current injection site in the soma to the distal part of AIS. By moving the starting point $x_{a}$ away from the 

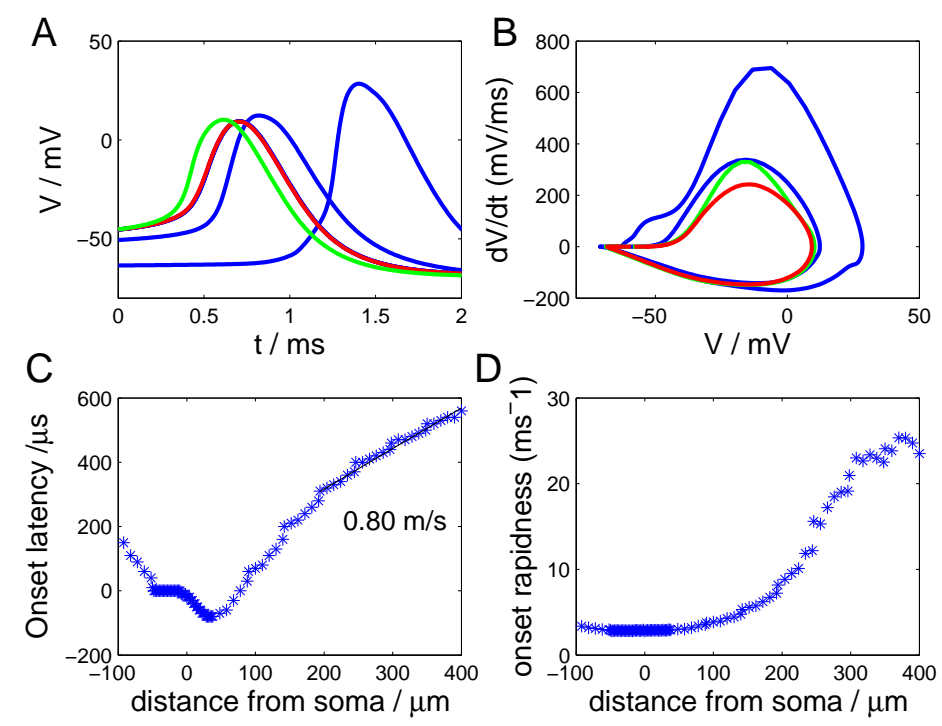

Figure 12.2: Simulation of neurons with active dendrites (A) APs evoked at different locations are superimposed on the same time axis. Green trace: AP at the axonal initial segment (AIS); red trace: AP at the somatic site; blue traces: APs at dendrites. (B) Phase plots depict the rate of change in membrane potential $\mathrm{d} V / \mathrm{d} t$ with respect to $V$ at the corresponding locations. (C) AP onset latency plotted over distance. (D) Onset rapidness of APs at different locations. $x_{a}=20 \mu \mathrm{m}, \bar{g}_{\mathrm{Na}}=900 \mathrm{pS} / \mathrm{\mu m}^{2}$.
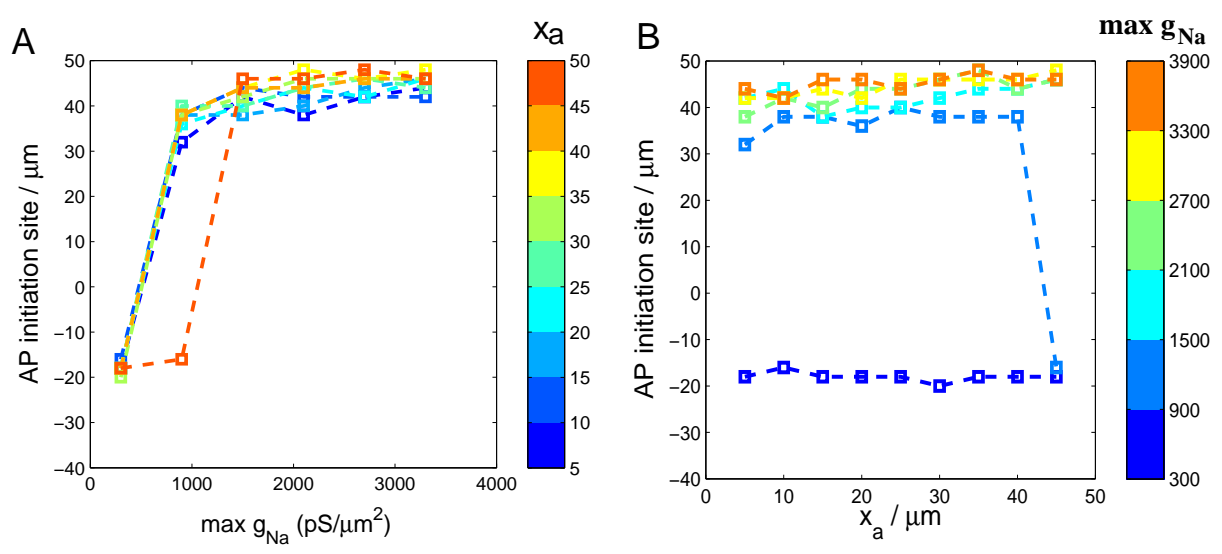

Figure 12.3: AP initiation sites in neurons with active dendrites (A) The sites of AP initiation shifted from soma ( -20 to $0 \mu \mathrm{m}$, with current injection at $10 \mu \mathrm{m})$ to the distal AIS (30 to $50 \mu \mathrm{m})$ as $\bar{g}_{\mathrm{Na}}$ increased above $900 \mathrm{pS} / \mathrm{\mu m}^{2}$ in the model. (B) Axonal AP initiation sites were preferentially at distal AIS (30-50 $\mu \mathrm{m})$ while varying the starting point of AIS between 5 and $45 \mu \mathrm{m}$. 
soma, the total membrane area of AIS was decreased, thus it required higher $\mathrm{Na}^{+}$channel density at AIS for an AP initiation(Figure 12.3A A, red trace).

On the other hand, when the AP was initiated at axon, a small positive correlation was detected between $x_{a}$ and the initiation site such that a larger $x_{a}$ resulted in an initiation site further away from the soma (Figure 12.3B). However, even if the AIS region started right after the soma $\left(x_{a}=5 \mu \mathrm{m}\right)$, the site of axonal AP initiation was found to be at the end of the AIS ( $30 \mu \mathrm{m}$ away from the axon hillock), which might be due to the fact that the distal AIS is electrically isolated from the current sink of soma and dendrites, such that the membrane at the distal AIS is most easily depolarized by local activation of $\mathrm{Na}^{+}$channels.

\subsubsection{Onset Dynamics of the Somatic AP}

From these simulations, we further analyzed the waveforms of the somatic AP, in particular its onset dynamics. As shown in Figure 12.4A, the onset rapidness of the somatic APs increased with the $\mathrm{Na}^{+}$channel density $\bar{g}_{\mathrm{Na}}$ at AIS. Such
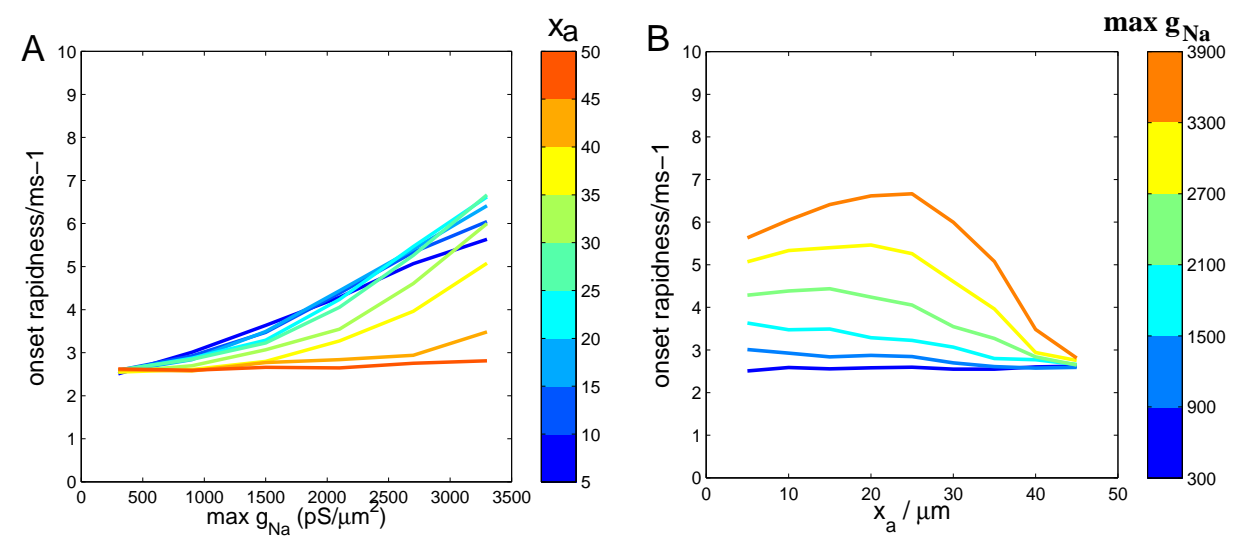

Figure 12.4: Somatic AP onset in neurons with active dendrite A The onset rapidness of somatic spikes increased slightly with enhancing $\bar{g}_{\mathrm{Na}}$ at AIS. B The changes of somatic AP onset while varying the starting point of AIS $x_{a}$ between $5 \mu \mathrm{m}$ and $45 \mu \mathrm{m}$ distance from soma.

increase was more pronounce when the AIS started at a closer distance $x_{a}$ away from the soma (Figure [12.4 $\mathrm{B}$ ). Our interpretation is that the total number of $\mathrm{Na}^{+}$channels at the membrane area between AP initiation site and the soma might account for the increase of onset rapidness at soma, due to more $\mathrm{Na}^{+}$current recruited along the way propagating from the initiation site to the soma. A larger propagation distance by increasing $x_{a}$ was compromised by the reduction of the total membrane area of AIS, resulting in an optimal distane of $x_{a}$, a value which was larger for higher $\mathrm{Na}^{+}$channel density. If the channel density was relatively 
low $\left(\bar{g}_{\mathrm{Na}} \leq 1500 \mathrm{pS} / \mathrm{\mu m}^{2}\right)$, the onset rapidness of the somatic AP was found to decrease monotonically with increasing $x_{a}$.

Above all, the somatic APs exhibited a smooth onset for the length of AIS between $5 \mu \mathrm{m}$ and $45 \mu \mathrm{m}$ and the $\mathrm{Na}^{+}$channel density at AIS ranging from 300 to $3900 \mathrm{pS} / \mathrm{um}^{2}$. The fastest onset we found in these simulations was far below $20 \mathrm{~ms}^{-1}$ as observed from the cortical neuron recordings.

\subsection{Models with Passive Dendrites}

To explore the potential impact of neuronal compartments distant from the AP initiation site, we generated models with a $3000 \mu \mathrm{m}$ long dendrite with much lower $\mathrm{Na}^{+}$channel density $\bar{g}_{\mathrm{Na}}=30 \mathrm{pS} / \mathrm{\mu m}^{2}$. The dendritic membrane was to the extreme so passive that it even failed to regenerate propagating APs. APs indeed only invaded the first $150 \mu \mathrm{m}$ of the model dendrites, which was about $5 \%$ of the total dendritic area in the model. In these models with passive dendrites, we studied the somatic AP waveforms varying $\bar{g}_{\mathrm{Na}}$ at AIS up to $6000 \mathrm{pS} / \mathrm{\mu m}^{2}$. Figure 12.5 shows a simulation example with $x_{a}=20 \mu \mathrm{m}$ and $\bar{g}_{\mathrm{Na}}=6000 \mathrm{pS} / \mathrm{um}^{2}$.
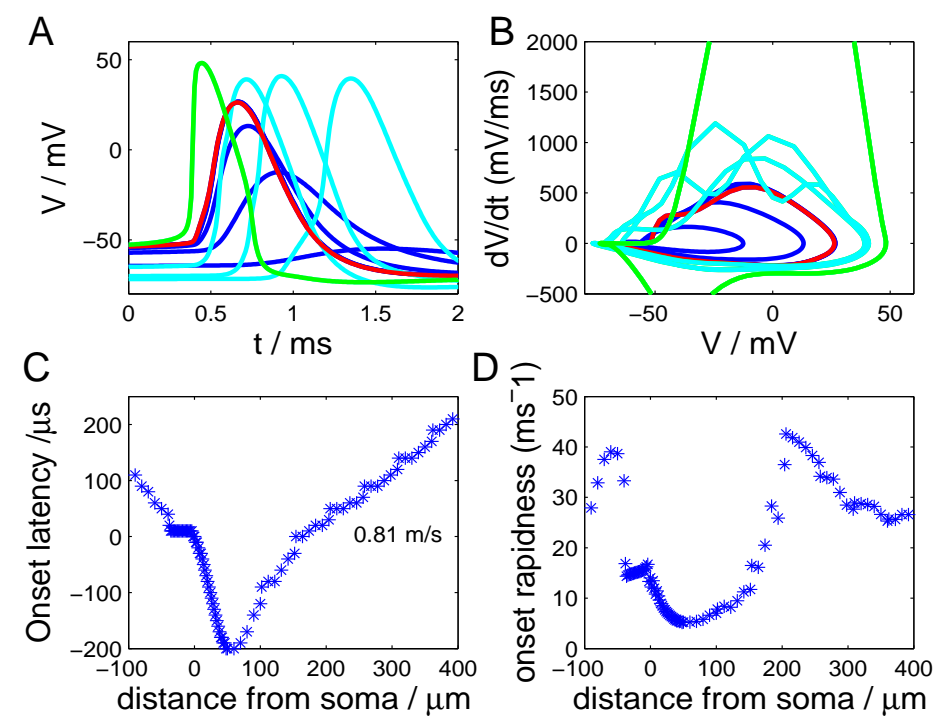

Figure 12.5: Simulation example of neurons with passive dendrite(A) APs evoked at different locations are superimposed on the same time axis: green trace: $\mathrm{AP}$ at the axon initial segment (AIS); red trace: AP at the somatic site; blue traces: APs at dendrites; cyan traces: APs propating down the axon. (B) Phase plots depict the rate of change in membrane potential $\mathrm{d} V / \mathrm{d} t$ with respect to $V$ at the corresponding locations. (C) AP Onset latency plotted over distance. (D) Onset rapidness of APs at different locations. $x_{a}=20 \mu \mathrm{m}, \bar{g}_{\mathrm{Na}}=6000 \mathrm{pS} / \mathrm{\mu m}^{2}$.

When we compared it to propagating APs with active dendrite in Figure 12.2, 
here the peak values of APs decreased dramaticly during back propagation (from green trace to red and to blue traces in Figure $12.2 \mathrm{~A}$ ). The AP waveforms became broader and smaller from the AIS back to soma and further into the passive dendrites.

Moreover, the peak rate of changes $\mathrm{d} V / \mathrm{d} t$ in membrane potentials at the AIS was unrealistically high being much larger than $2000 \mathrm{~V} / \mathrm{s}$ (Figure 12.2B). Such a high value of $\mathrm{d} V / \mathrm{d} t$ has never been observed in experimental recordings but resulted in the generation of double peaks in the phase plots of APs propagating down the axon (cyan trances in Figure 12.2B).

From Figure 12.5C the onset latency between the AP initiation site (around $50 \mu \mathrm{m}$ ) and the soma was as large as $200 \mu \mathrm{s}$. The propagation speed of APs along the axon was also smaller compared to the simulations with active dendrites.

The onset rapidness recorded between the AIS and the somatic site was not a flat function any more, As shown in Figure 12.5D, the AP onset became faster at both the left and right sides of the AP initiation site. The value of onset rapidness increased abruptly at the transition point between the soma and the dendrites. The somatic AP shows an onset rapidness of about $15 \mathrm{~ms}^{-1}$ in this simulation example.

\subsubsection{Sites of AP Initiation}

The AP initiation sites in the model with passive dendrite were no longer restricted to the soma and the distal AIS. As in the model with active dendrites, neurons with homogeneous channel density $\bar{g}_{\mathrm{Na}}$ across the soma and the AIS showed AP initiation at the soma; neurons with much higher channel density at the AIS and a relatively smaller value of $x_{a}$ showed AP initiation at the distal AIS. Given an intermediate $\bar{g}_{\mathrm{Na}}$ at the AIS, the models with passive dendrites showed AP initiation at the proximal axon, which was sometimes even before the starting point of the AIS. The sites of AP initiation moved closer to the soma while varying the starting point of AIS $x_{a}$ away from the soma (Figure 12.6).

This result seems counter-intuitive at first glance. Our interpretation is as follows. The preference for AP initiation at the AIS compared to that at the soma was due to either a higher $\mathrm{Na}^{+}$channel density at the AIS, or an electrical isolation of the axon from the large current sink induced by the long passive dendrite. When $\bar{g}_{\mathrm{Na}}$ at the AIS was high enough, both of the effects resulted in an AP initiation at the distal part of AIS. However, due to the large current sink induced by the long passive dendrites, if $\bar{g}_{\mathrm{Na}}$ at the AIS was not high enough and the membrane area of the AIS was relatively smaller with a larger value of $x_{a}$, the local activation of $\mathrm{Na}^{+}$channels per se was not sufficient to trigger an AP. Thus the AP initiation was induced at the proximal axon which was strongly depolarized by the current injection at the soma. 

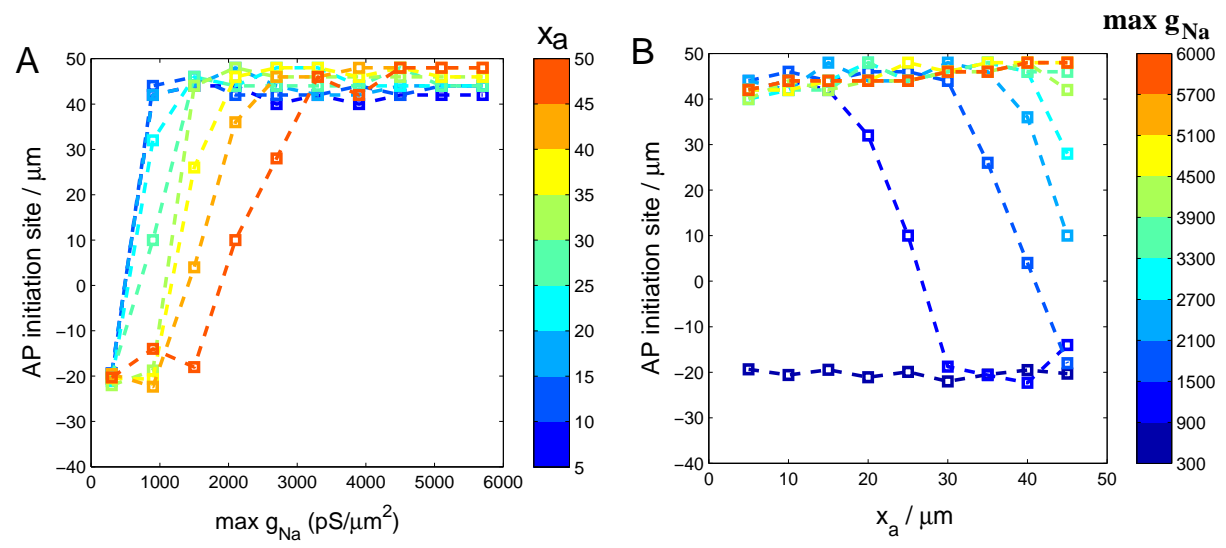

Figure 12.6: AP initiation site in neurons with passive dendrites (A) The site of AP initiation moved from the somatic site $(-20$ to $0 \mu \mathrm{m}$, with current injection at $10 \mu \mathrm{m}$ ) to the distal AIS as $\bar{g}_{\mathrm{Na}}$ at the AIS increased; (B) The sites of AP initiation while varying the starting point of AIS $x_{a}$ away from the soma; Color code indicates the $\mathrm{Na}^{+}$channel density at the AIS (For a detailed explanation see the text).

\subsubsection{Onset Dynamics of the Somatic AP}

The onset dynamics of the somatic APs were analysed in the models with passive dendrites. For all those simulations with AP initiation at the distal AIS, the onset of the somatic APs speeded up significantly with increasing $\bar{g}_{\mathrm{Na}}$ at AIS (Figure [12.7A). On the other hand, if the initiation site was close to the soma (i.e., compare the red trace in Figure 12.6 and that in Figure 12.7A ), the somatic APs exhibited a smooth onset far below $5 \mathrm{~ms}^{-1}$. Given the AIS starting at 30 to $40 \mu \mathrm{m}$ away with a channel density of $5000 \mathrm{pS} / \mathrm{\mu m}^{2}$, the somatic AP onset could be as fast as $20 \mathrm{~ms}^{-1}$.

The onset rapidness of the somatic APs versus the starting point $x_{a}$ of the AIS peaked at intermediate values of $x_{a}$. When $x_{a}$ was small, the propagation distance from the AIS to the soma was correspondingly small; When $x_{a}$ was large, the total membrane area of the AIS was reduced and thus less $\mathrm{Na}^{+}$channels were activated during the period of backpropagation from the AP initiation site to the soma. As $\bar{g}_{\mathrm{Na}}$ at AIS increased, the optimal value of $x_{a}$ was shifted to a more distal site (Figure 12.7B).

To summarize the parameter dependence of the AP initiation site and the onset rapidness of the somatic APs, we presented the simulation results of our models with passive dendrites by the phase diagrams shown in Figure 12.8. The AP initiation sites were found mostly at the distal AIS $(L>40 \mu \mathrm{m})$ given the $\mathrm{Na}^{+}$channel density $\bar{g}_{\mathrm{Na}}>1000 \mathrm{pS} / \mathrm{\mu m}^{2}$ at the AIS. Only if the $\mathrm{Na}^{+}$channel density was not so high at the AIS or the total membrane area of the AIS was 

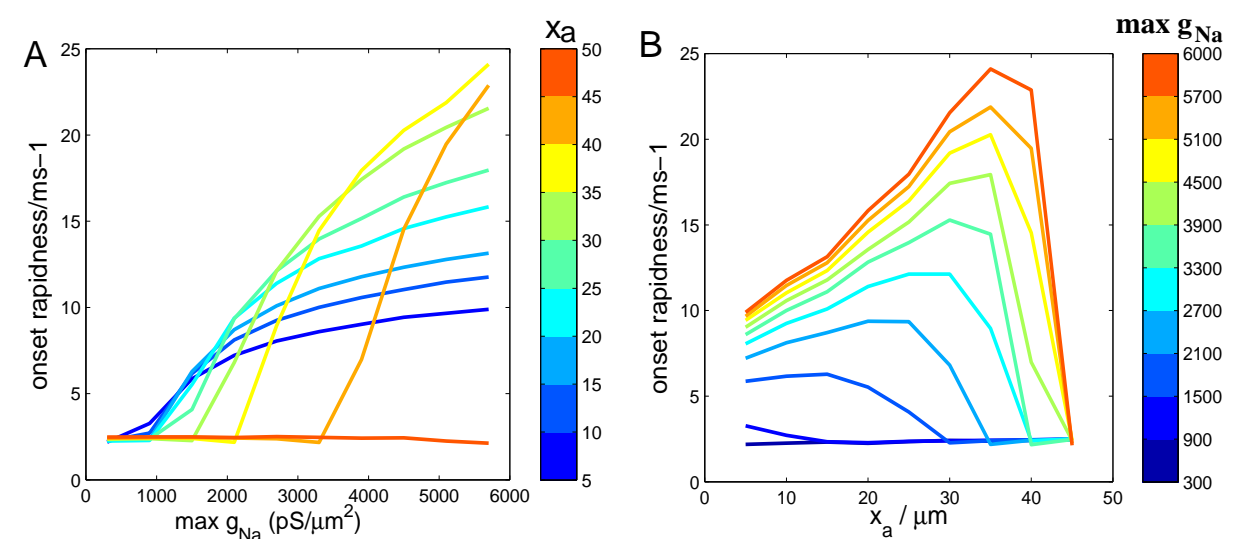

Figure 12.7: Somatic AP onset in neurons with passive dendrite (A) The onset rapidness of somatic spikes increased significantly with enhancing $\bar{g}_{\mathrm{Na}}$ at AIS. (B) The changes of somatic AP onset while varying the starting point of AIS $x_{a}$ between $5 \mu \mathrm{m}$ and $45 \mu \mathrm{m}$ away from soma. The maximum onset speed of somatic spikes was induced by intermediate values of $x_{a}$, which was shifted to a larger value as $\bar{g}_{\mathrm{Na}}$ increased.

relatively small, the APs were found to initiate at the soma or the proximal AIS (Figure 12.8A). The onset rapidness of the somatic APs is shown in Figure 12.8B.
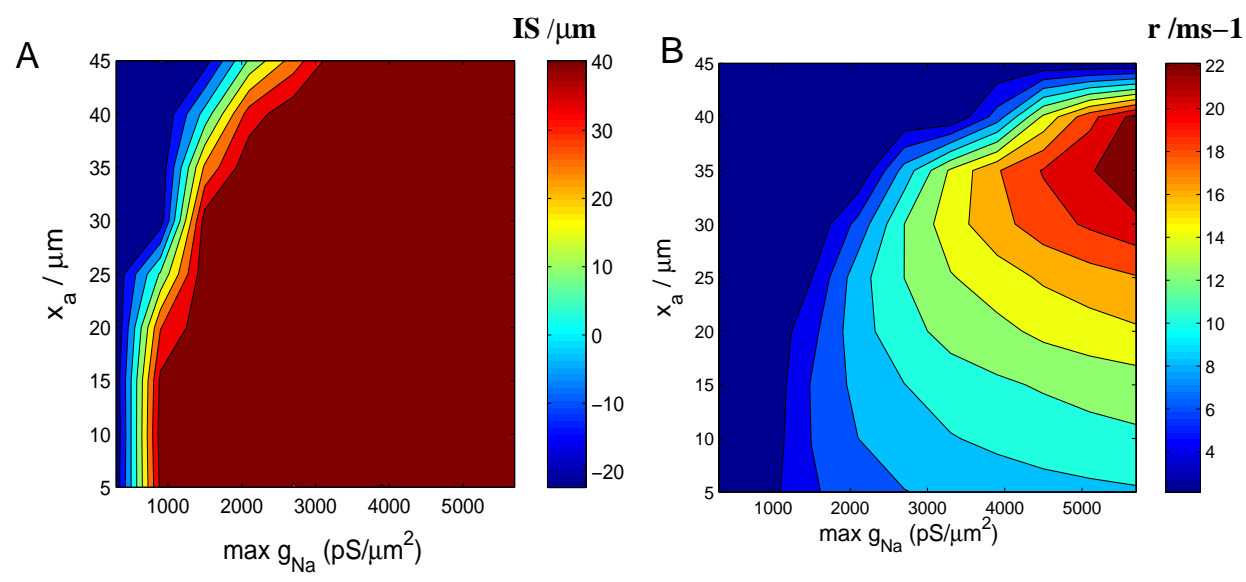

Figure 12.8: Phase diagram in models with passive dendrites (A) AP initiation site and (B) onset rapidness of the somatic APs in simulations varying the starting point $x_{a}$ of the AIS and its $\mathrm{Na}^{+}$channel density.

The changes of the somatic waveforms induced by propagation became more pronounce with increasing $\bar{g}_{\mathrm{Na}}$ at the AIS and the starting point $x_{a}$ of the AIS further away from the soma. Among these models the fastest onset of the somatic 
APs was found in the simulation with $\bar{g}_{\mathrm{Na}}=6000 \mathrm{pS} / \mu \mathrm{m}^{2}$ and $x_{a}=35 \mu \mathrm{m}$, yielding a value of $r=24 \mathrm{~ms}^{-1}$.

\subsection{Impact on Dynamic Response Properties}

What are the response properties of a spatially extended neuron model with somatic current injection and axonal AP initiation? In the following, we investigate the voltage response at the soma and the AIS in the presence of synaptic noise. The voltage traces at these two sites revealed a highly linear correlation. The corresponding population response function is compared to those in the single compartment neuron models.

\subsubsection{Voltage Dynamics at the Soma and the AIS}

To study a neuron's input-output function to convert fluctuating synaptic inputs into spike trains, we simulated the multi-compartment neuron models applying time-varying inputs at the soma:

$$
I(t)=I_{0}+\sigma_{I} I_{\mathrm{syn}}(t)
$$

where the synaptic noise $I_{\text {syn }}(t)$ was generated from a stochastic Ornstein-Uhlenbeck process (see Chapter 9.1).

Figure 12.9]A shows the voltage traces at the soma and the AIS in models with a 10-fold increase of $\mathrm{Na}^{+}$channel density at the AIS compared to the soma. Different from repetitive firing under the constant current injection, the membrane potentials showed subthreshold fluctuation interrupted by firing of one or more action potentials. Due to the high channel density, action potentials were first generated at the AIS and then propagated into soma with an onset latency of around 200 us (Figure 12.9A, inset).

The phase plots in Figure $12.9 \mathrm{~B}$ shows a monophasic waveform at the AP initiation site with a smooth onset of about $4 \mathrm{~ms}^{-1}$. The somatic APs exhibit biphasic waveforms in the phase plot and the onset speed is slightly faster, with a value around $7 \mathrm{~ms}^{-1}$. Given the maximum $\mathrm{Na}^{+}$channel conductance $\bar{g}_{\mathrm{Na}}$ at the AIS was $3500 \mathrm{pS} / \mathrm{\mu m}^{2}$, such as 10 -fold of the somatic $\mathrm{Na}^{+}$channel density, the peak rate of MP rise $d V / d t$ at the AIS was as high as $2000 \mathrm{~V} / \mathrm{s}$.

We computed the variances of the subthreshold MP fluctuations at the soma and the AIS, as shown in Figure 12.9C. To erase memory effects from the previous spikes, we used only time windows starting from $30 \mathrm{~ms}$ after each spike to the onset of the next spike. The subthreshold membrane potential fluctuation at the soma and at the AIS showed a highly linear correlation in all models we investigated.

Figure 12.9D plots the voltage thresholds of AP onsets at both sites, which also suggests a strongly linear correlation. The voltage thresholds at the soma 
were more depolarized and showed a slightly smaller variability than those at the AIS.
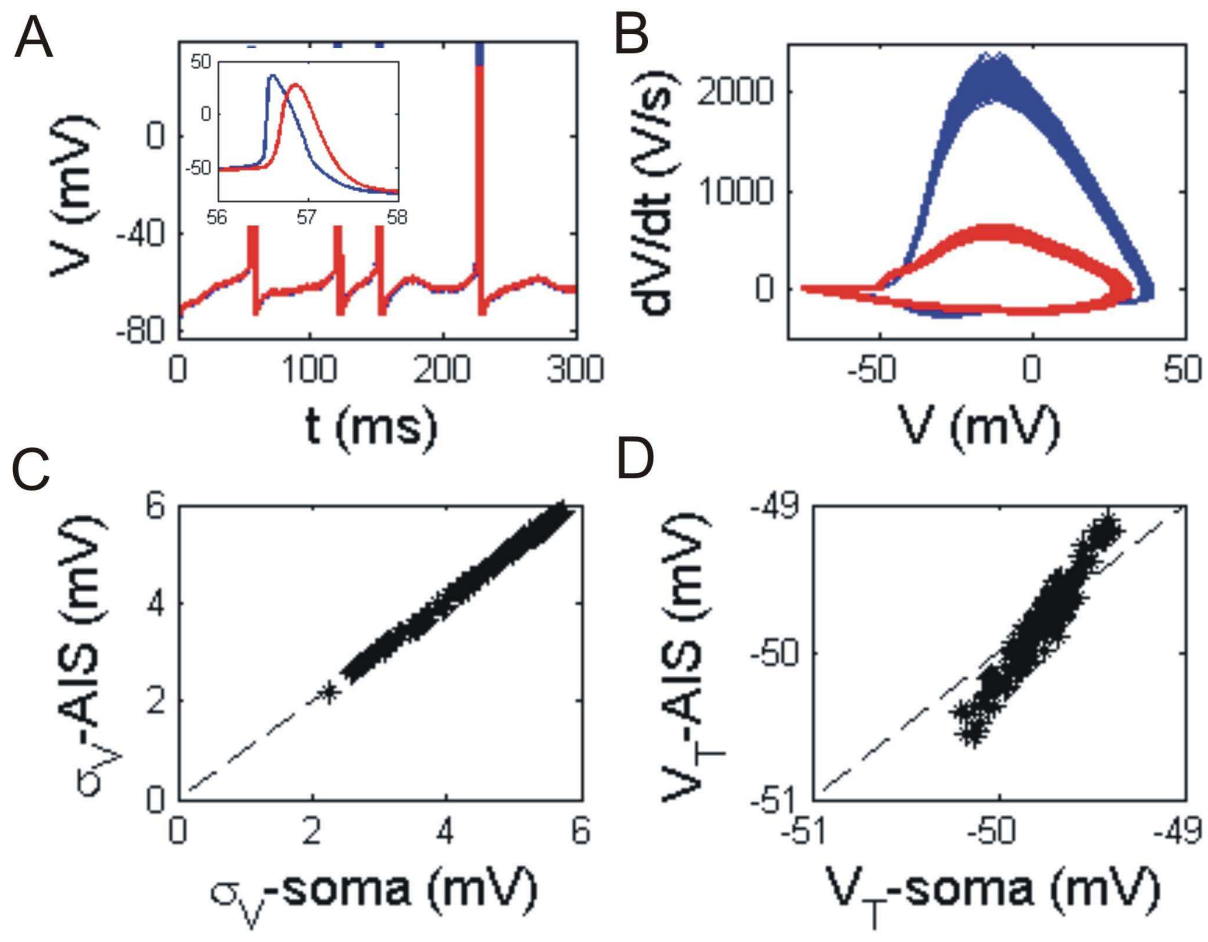

Figure 12.9: Response to noisy inputs in models with $\bar{g}_{\mathrm{Na}} \times 10$ at AIS. The model with active dendrites: $\bar{g}_{\mathrm{Na}}=300 \mathrm{pS} / \mathrm{\mu m}^{2}$ at the soma; $\bar{g}_{\mathrm{Na}}=3000$ $\mathrm{pS} / \mathrm{\mu m}^{2}$ at the AIS; $x_{a}=30 \mu \mathrm{m}$. The injected current was generated from an Ornstein-Uhlenbeck stochastic process with $\tau_{c}=40 \mathrm{~ms}$. (A) The voltage traces at the soma (red) and the AIS (blue). Inset: the enlarged window of one AP indicating axonal initiation of spikes. (B) The phase plots showing the rate of changes in membrane potentials v.s. the voltages at the soma (red) and the AIS (blue). Inset: the enlarged phase plot of the somatic APs. (C) Correlation of the sub-threshold membrane potential fluctuations at the soma and the AIS. (D) Correlation of the voltage thresholds at the soma and the AIS. The total simulation time: $20 \mathrm{~s}$; time step: $10 \mu \mathrm{s}$.

\subsubsection{Frequency Response to Oscillatory Noisy Inputs}

Finally, we compared the coding properties of these multi-compartment neuron models with those of point neuron models. We simulated the multi-compartment models by somatic injection of noisy oscillatory inputs

$$
I(t)=I_{0}+I_{1} \cos (2 \pi f t)+\sigma_{I} I_{\mathrm{syn}}(t),
$$




\section{Dynamics of AP Initiation in the Soma-Proximal Axon-Complex}

The constant current level $I_{0}$ was adjusted in simulations to set the mean firing rate $\nu_{0}$ around $10 \mathrm{~Hz}$. The modulation of the input currents $I_{1}$ was taken as $10 \%$ of $I_{0}$. The magnitude of the synaptic noise $\sigma$ was chosen such that the subthreshold fluctuation of somatic MP had a standard deviation between 3 and $5 \mathrm{mV}$ and the temporal correlation of noisy current has a time constant of $40 \mathrm{~ms}$.

The population response of these model neurons was characterized by the instantaneous firing rate $\nu(t)$ averaged over thousands of trials and fitted by the linear response function

$$
\nu(t)=\nu_{0}+\nu_{1} \cos (2 \pi f t+\phi(f)) .
$$

The frequency modulation $\nu_{1}$ of the population firing rate was plotted against the input frequency in Figure 12.10 .

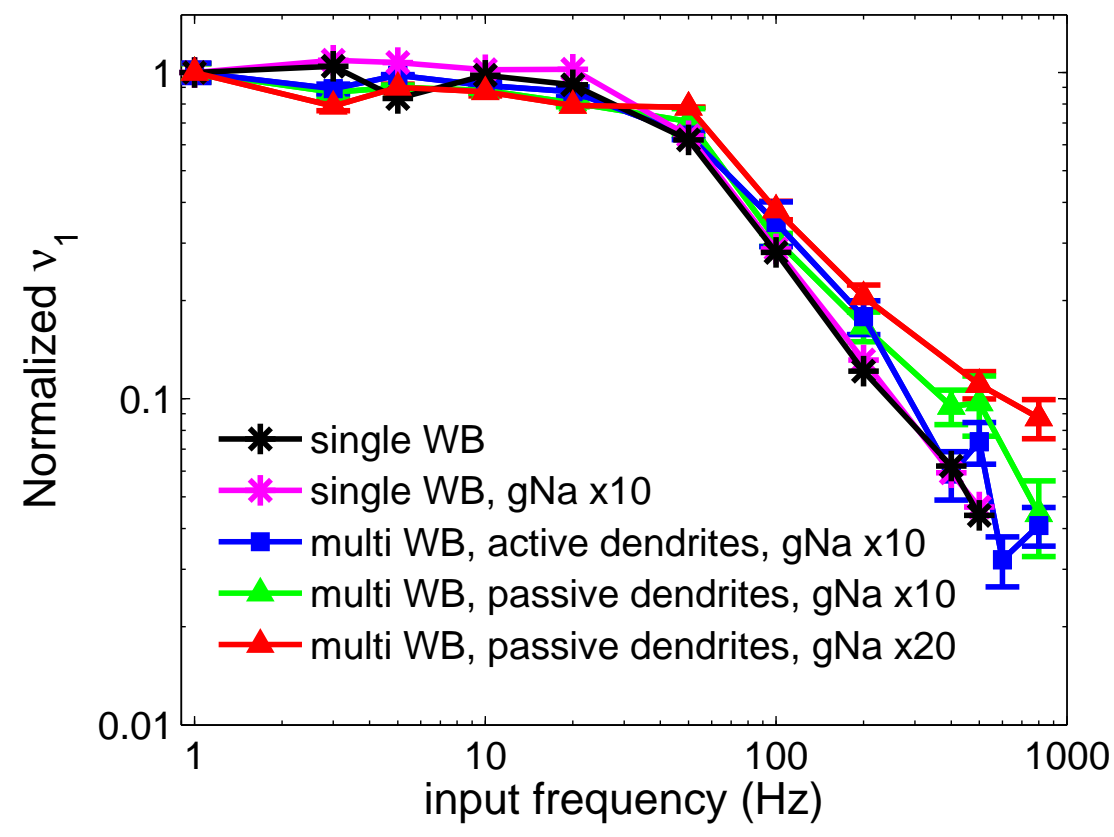

Figure 12.10: Frequency modulation of Population Firing Rate in multicompartment models. Asterisk: single-compartment Wang-Buzsaki models with $\bar{g}_{\mathrm{Na}}=300 \mathrm{pS} / \mathrm{\mu m}^{2}$ (black) and $\bar{g}_{\mathrm{Na}}=3000 \mathrm{pS} / \mathrm{\mu m}^{2}$ (magenta). Squares: multi-compartment Wang-Buzsaki models with active dendrites; $\bar{g}_{\mathrm{Na}}=300$ $\mathrm{pS} / \mathrm{\mu m}^{2}$ at the soma and $\bar{g}_{\mathrm{Na}}=3000 \mathrm{pS} / \mathrm{\mu m}^{2}$ at the AIS. Triangles: multicompartment Wang-Buzsaki models with large passive dendrites; $\bar{g}_{\mathrm{Na}}=300$ $\mathrm{pS} / \mathrm{\mu m}^{2}$ at the soma, $\bar{g}_{\mathrm{Na}}=3000 \mathrm{pS} / \mathrm{\mu m}^{2}$ (green) and $6000 \mathrm{pS} / \mathrm{\mu m}^{2}$ (red) at the AIS. The temporal correlation of the background synaptic noise $\tau_{c}=40 \mathrm{~ms}$. Error bars represent the standard error of the mean (SEM) across independent trials. 
From Figure 12.10, the strength of the oscillatory response $\nu_{1}$ in these multicompartment models showed similar damping at high input frequency as was found in the single-compartment Hodgkin-Huxley-type models. In the presence of synaptic noise with $\tau_{c}=40 \mathrm{~ms}$, the cutoff frequency was about $50 \mathrm{~Hz}$ in both models. For input frequencies $f \geq 200 \mathrm{~Hz}$, the response modulation $\nu_{1}$ went up significantly when we increased the $\mathrm{Na}^{+}$channel density at AIS from $300 \mathrm{pS} / \mathrm{\mu m}^{2}$ to $3000 \mathrm{pS} / \mathrm{\mu m}^{2}$ and $6000 \mathrm{pS} / \mathrm{\mu m}^{2}$ in the passive dendrite models. On the contrary, a 10-fold increase of $\mathrm{Na}^{+}$channel density in single compartment models and active dendrite models has rarely any impact on the modulation amplitude of high frequency response.

\subsection{Summary and Discussion}

In this chapter, we studied two classes of multi-compartment models of myelinated neurons using physiologically constrained parameters. The onset dynamics of somatic APs were analyzed in simulations while systematically varying the length of the AIS and its $\mathrm{Na}^{+}$channel density. In models with homogenous channel distribution across soma and dendrites, the somatic AP onset was as smooth as that at the AP initiation sites; In models with large passive dendrites where APs only invaded $5 \%$ of the total dendritic membrane, the somatic AP waveforms were strongly shaped by lateral currents in the parameter regime with a distal AIS and a 10-fold or even higher $\mathrm{Na}^{+}$channel density at the AIS.

Different from the single compartment neuron models, the input and output sites are spatially separated in real neurons, where soma is considered to be the integration site of synaptic inputs, and AIS is the initiation site of action potentials. How is the neurons' output function affected by the spatial isolation of soma and AIS in the multi-compartment models?

We have shown above that in most physiological models there are no significant differences between soma and AIS in AP onset dynamics. Due to the fact that APs are initiated in the AIS, the proximal site of the axon, which is electrically tightly coupled to the soma, subthreshold MP fluctuations of these two sites are found to be highly correlated. Our simulations further predict a strong linear correlation of the voltage thresholds at both sites (Figure 12.9). These results demonstrated that it is plausible to predict the spike generation at AIS from the temporal dynamics of the MP fluctuations at soma.

Intriguingly, although dendrites are often treated as the sites of synaptic inputs, our study suggests that the morphology of the dendritic tree has impact on the somatic AP waveform and the output function of the neuron. The somatic $\mathrm{AP}$ onset could reach the observed value of about $20 \mathrm{~ms}^{-1}$ in models with large passive dendrite if the $\mathrm{Na}^{+}$channel density at AIS was as high as $5000 \mathrm{pS} / \mathrm{\mu m}^{2}$. However, so far no direct experimental evidences support the existence of large passive dendritic trees and extremely high $\mathrm{Na}^{+}$channel density at AIS. Thus an 


\section{Dynamics of AP Initiation in the Soma-Proximal Axon-Complex}

overwhelmingly strong lateral current is not very likely to occur in AP initiation dynamics of cortical neurons.

We also computed the response function to noisy input of different frequencies in the spatially-extended neuron models. There seemed to be a small but significant improvement of the high frequency response in these models with passive dendrites compared to the point neuron model and the models with active dendrites. Nevertheless, all the multi-compartment models we have investigated exhibited a power-law-like decay of the modulation amplitude for input frequency $f>50 \mathrm{~Hz}$, contradicting to the experimental observation of undamped responses up to $200 \mathrm{~Hz}$ in cortical neurons (Köndgen et al., 2008). 


\section{Chapter 13}

\section{Discussion and Conclusion of Part II}

In this study, we characterized the phase plot dynamics of action potentials in different biophysical models of cortical neurons and explored the population coding properties of these models. A new class of $\mathrm{CB}$ model called the cooperative Wang-Buzsaki (cWB) model was constructed by implementing the mechanism of channel cooperativity. This model including a small fraction of strongly coupled $\mathrm{Na}^{+}$channels exhibited an abrupt AP onset and a substantial improvement of its high frequency response. Our comprehensive simulations of multi-compartment models suggested that phase plots of somatic APs largely represented the dynamics of AP initiation at AIS. Exceptionally, neuron models with large passive dendrites and an extremely high $\mathrm{Na}^{+}$channel density at the AIS showed a fast onset dynamics and biphasic phase plots. These models appeared slightly more sensitive to high frequency inputs with increasing of $\mathrm{Na}^{+}$density at the AIS. Thus our results suggested two possible mechanisms in modeling of cortical neurons with improved high-frequency coding properties.

\section{Do phase plots of somatic APs represent the dy- namics of AP generation?}

In the past years, many studies have used phase plots from somatic recordings to characterize the dynamics of AP initiation, however, the interpretation of the observed somatic AP waveforms is still an open question. In many spatially extended neuron models we investigated in this study, phase plots obtained from somatic APs provide a valid tool to infer the characteristic $\mathrm{Na}^{+}$current activation. We performed systematic simulations of multi-compartment neuron models varying the AP propagation distance, the soma size, and the $\mathrm{Na}^{+}$channel density. Different models of Hodgkin-Huxley-type channel kinetics showed qualitatively the same parameter dependence of the AP onset dynamics. Even the Wang- 
Buzsaki model with the fastest conceivable $\mathrm{Na}^{+}$channel activation kinetics failed to reproduce a rapid onset of somatic APs as recorded in cortical neurons. In these modes, the somatic AP waveforms remained as smooth as at the AP initiation site under the physiological constraint that the propagation time from AIS to soma was consistent with experimental observations and for a large range of channel distributions.

We found two special situations where the AP waveform at the soma was strongly influenced by lateral currents: this can occur either for antidromic APs with a long propagation distance, or by APs initiated at the distal AIS back propagating into soma connected to a large passive dendrite.

In the first case, the antidromic APs should propagate over a distance at least one electrotonic length in order to establish a strong potential field in space and thus large lateral currents. Previous studies have provided substantial experimental evidence that action potentials of the pyramidal neurons are initiated in the axon initial segment (AIS). 30-40 um awav from the soma (Palmer \& Stuart, 2006; Kole et al., 2007; Meeks \& Mennerick, 2007). In our simulations the passive parameters were chosen such that the electrotonic length of the cable was $\lambda \simeq 400 \mathrm{um}$. as suggested bv simultaneous recordings from soma and axon (Shu et al., 2006). With this parameter choice, if the spike is triggered within $50 \mu \mathrm{m}$ away from the soma, the onset dynamics of somatic APs was as slow as that at the initiation site. We conclude that antidromic propagation per se cannot explain the observed high onset rapidness if the AP initiation site is as close to the soma as currently believed.

In the second situation, neurons with a large passive dendrite could strongly shape the onset dynamics of the somatic APs. One example of such a model has been recently described by $\mathrm{Yu}$ et al. (2008). Such a passive dendrite contains few $\mathrm{Na}^{+}$channels not even enough to regenerate back-propagating APs. Indeed APs invaded only a small amount of dendritic area, which covered $5 \%$ of the total dendritic area in our simulation. However, physiological studies have revealed similar $\mathrm{Na}^{+}$channel densities across soma and dendrites (Huguenard et al., 1989; Stuart \& Sakmann, 1994; Kole et al., 2008). Dendritic membrane was found to be rather excitable in many studies of dendritic APs such that backpropagating APs spread effectively throughout the dendritic tree (Stuart \& Sakmann, 1994; Magee \& Carruth, 1999; Williams \& Stuart, 2000). Due to the large current sink provided by passive dendrites, APs in those models were delayed before propagating into the soma, which apparently leads to a large lateral current and a change in somatic AP waveform. As a consequence, such a model yielded an extremely high rate of rise of MPs at the initiation sites, with a rather value up to $3000 \mathrm{~V} / \mathrm{s}$. In contrast, the experimentally recorded peak rate of rise $\mathrm{d} V / \mathrm{d} t$ at axon is about $1000 \mathrm{~V} / \mathrm{s}$ or even below. Our study of passive dendrite models thus resulted in a rather unrealistic situation.

Except for the two situations discussed above, in most cases phase plots of somatic APs reveal the characteristics of the AP generator. A recent study by 
(Badel et al., 2008) used the dynamic $I-V$ curves obtained from phase plots of experimental recordings to generate abstract neuron models, which very precisely predicted both the subthreshold and super-threshold responses of the neuron to novel stimuli. The success of these predictions is hardly understandable if the onset dynamics of the somatic AP is fundamentally different from that in the initiation site. Our results showed the congruence of the AP onset dynamics in the soma and in the AIS, justifying the use of phase plots from somatic recordings to predict a neuron's output function.

\section{What makes cortical neurons sensitive to high fre- quency inputs?}

To explore the mechanism in the spike generation of cortical neurons that facilitates the fast response of a cortical neuronal population, we computed the neurons' response function to noisy inputs of various frequencies in different conductance-based models.

Our study characterized the AP onset dynamics and response function in a new class of CB models for AP generation, implementing the mechanism of channel cooperativity. Cooperativity of channel gating has been reported in studies of various types of ion channels Molina et al. 2006: Marx et al. 2001: Undrovinas et al., 1992). Only recently, Naundorf et al. (2006) hypothesized that cooperative gating of $\mathrm{Na}^{+}$channels may underly the observed abrupt AP onset in cortical neurons. Our results directly demonstrate that the cooperative Wang-Buzsaki (cWB) model exhibits a rapid AP onset and two distinct components in the phase plots, resulting from collective activation of the cooperative channel fraction. Given $10 \%$ of cooperative $\mathrm{Na}^{+}$channels with strong coupling strength, the modulation amplitude of the neuronal response at input frequency $f>200 \mathrm{~Hz}$ is about one order of magnitude larger than that in the uncoupled models (Figure 10.7). The modulation gain at high input frequency decays roughly exponentially, which deviates somewhat from the power law behaviors previously reported in conventional conductance-based models (Knight, 1972a, b; Brunel et al.. 2001: Fourcaud-Trocmé et al.. 2003: Naundorf et al.. 2005a: Badel et al., 2008).

Because there exists an alternative explanation of the rapid somatic AP onset by the 'lateral current hypothesis', we further study the frequency-dependent encoding in spatially extended neuron models with noisy current injection to the soma and AP initiation in the AIS. How is a neuron's response function influenced by the spatial displacement of the input and output sites in real neurons? Our study computed for the first time the frequency response function in multi-compartment neuron models. The high frequency response of these models showed power-law decay at input frequencies $f>50 \mathrm{~Hz}$ (Figure 12.10) similar 
to the behaviors found in the Hodgkin-Huxley-type single compartment models. However, the response strength slightly increased with increasing of $\mathrm{Na}^{+}$channel density at the AIS, resulting in a partial undamping of the high frequency response. To the contrary, a 10-fold increase of $\mathrm{Na}^{+}$channel density in the single compartment neuron models had no effect on the high frequency response. Our results suggest a small but significant improvement of the high frequency response in multi-compartment models with increasing $\mathrm{Na}^{+}$channel density at AIS.

Recently, Köndgen et al. (2008) reported for cortical neurons a rather flat frequency response function with no attenuation of the modulation amplitude up to $200 \mathrm{~Hz}$ (see Figure 9.3.2). Despite the significant improvement of high frequency coding properties, neither of the model neurons we discussed above is yet in full agreement with the cortical neurons with respect to their capability of tracking fast-varying inputs.

\section{Conclusion}

The spatio-temporal dynamics of AP initiation and the response properties of cortical neurons has become very prominent over the past years. Recent experimental observation of abrupt onset and fast response of cortical APs (Silberberg et al., 2004; Naundorf et al., 2006; Köndgen et al., 2008) have been inspired by previous theoretical studies that explored the coding properties of simplified neuron models and their relation to the underlying spike generation mechanism Fourcaud-Trocmé et al. 2003: Fourcaud-Trocmé \& Brunel. 2005: Naundorf et al., 2005a, b). Intriguingly, so far no conductance-based model has been found that simultaneously reproduces the onset dynamics and coding properties of cortical neurons.

Our study identifies two different ways to construct such a neuron model more sensitive to fast varying inputs. First, in the cWB models with cooperative channel gating, a small fraction of strongly coupled $\mathrm{Na}^{+}$channels induces abrupt changes of AP initiation and biphasic features in phase plots. The high frequency responses of these models are substantially improved compared to the uncoupled models. Second, most multi-compartment models we have studied behave similarly as the single compartment models, such that phase plots of somatic APs are representative of the activation kinetics of the axonal AP generator. However, in neuron models with large passive dendrites and an extremely high $\mathrm{Na}^{+}$channel density at AIS, phase plots of somatic APs exhibit rapid onset and two distinct components as those in experimental recordings. These models with higher $\mathrm{Na}^{+}$channel density at AIS showed a slightly slower gain attenuation in the high frequency sensitivity.

In both models there appears to be a correlation between the rapid onset dynamics of somatic APs and improved high frequency response properties. Apparently, this correlation is due to entirely different underlying mechanisms in 
the two models. Further studies are thus required to clarify the relation of onset rapidness and dynamical response properties in $\mathrm{CB}$ models.

Our simulation results indicate that neither cooperative channel gating nor the spatial separation of input integration site (soma) and spike initiation site (AIS) can easily reproduce the response properties of cortical neurons with no attenuation up to the input frequency of $200 \mathrm{~Hz}$. This shows that the requirement to reproduce dynamic response functions of cortical neurons puts very tight constraints on biophysical models of these neurons, a test which most ad hoc models will not pass. Based on our results, it is tempting to hypothesize that both mechanisms might synergistically act on the functions of cortical neurons and in concert contribute to the coding properties for high frequency inputs. Whether this is the case or not is an interesting question for the future study. 


\section{Bibliography}

Azouz, R. \& Gray, C. M. (1999). Cellular mechanisms contributing to response variability of cortical neurons in vivo. J. Neurosci., 19, 2209-2223.

Badel, L., Lefort, S., Brette, R., Petersen, C. C., Gerstner, W., \& Richardson, M. J. (2008). Dynamic i-v curves are reliable predictors of naturalistic pyramidal-neuron voltage traces. J Neurophysiol, 99, 656-666.

Baranauskas, G. \& Martina, M. (2006). Sodium currents activate without a hodgkin-and-huxley-type delay in central mammalian neurons. J Neurosci, 26, 671-684.

Bean, B. P. (2007). The action potential in mammalian central neurons. Nat Rev Neurosci, 8, 451-465.

Bray, D. \& Duke, T. (2004). Conformational spread: the propagation of allosteric states in large multiprotein complexes. Annu Rev Biophys Biomol Struct, 33, 53-73.

Brunel, N., Chance, F. S., Fourcaud, N., \& Abbott, L. F. (2001). Effects of synaptic noise and filtering on the frequency response of spiking neurons. Phys Rev Lett, 86, 2186-2189.

Buzsáki, G. \& Draguhn, A. (2004). Neuronal oscillations in cortical networks. Science, 304, 1926-1929.

Clark, B. A., Monsivais, P., Branco, T., London, M., \& Häusser, M. (2005). The site of action potential initiation in cerebellar purkinje neurons. Nat Neurosci, 8, 137-139.

Colbert, C. M. \& Pan, E. (2002). Ion channel properties underlying axonal action potential initiation in pyramidal neurons. Nat Neurosci, 5, 533-538.

Dayan, P. \& Abbott, L. (2001). Theoretical neuroscience: computational and mathematical modeling of neural systems. (MA: The MIT Press). 
Destexhe, A. \& Paré, D. (1999). Impact of network activity on the integrative properties of neocortical pyramidal neurons in vivo. J Neurophysiol, 81, 15311547.

Eccles, J. C., Libet, B., \& Young, R. R. (1958). The behaviour of chromatolysed motoneurones studied by intracellular recording. J Physiol, 143, 11-40.

Engel, D. \& Jonas, P. (2005). Presynaptic action potential amplification by voltage-gated na + channels in hippocampal mossy fiber boutons. Neuron, 45, $405-417$.

Fourcaud-Trocmé, N. \& Brunel, N. (2005). Dynamics of the instantaneous firing rate in response to changes in input statistics. J Comput Neurosci, 18, 311-321.

Fourcaud-Trocmé, N., Hansel, D., van Vreeswijk, C., \& Brunel, N. (2003). How spike generation mechanisms determine the neuronal response to fluctuating inputs. J Neurosci, 23, 11628-11640.

Gardiner, C. (1994). Handbook of Stochastic Methods. (Springer Verlag).

Giugliano, M., Arsiero, M., Darbon, P., Streit, J., \& H.-R., L. (2006). Emerging network activity in dissociated cultures of neocortex: novel electrophysiological protocols and mathematical modelling. Kluwer/Plenum Press (USA).

Goldstein, S. S. \& Rall, W. (1974). Changes of action potential shape and velocity for changing core conductor geometry. Biophys J, 14, 731-757.

Greenberg, D. S., Houweling, A. R., \& Kerr, J. N. (2008). Population imaging of ongoing neuronal activity in the visual cortex of awake rats. Nat Neurosci, 11, 749-751.

Grill, W., Cantrell, M., \& Robertson, M. (2007). Antidromic propagation of action potentials in branched axons: implications for the mechanisms of action of deep brain stimulation. J Comput Neurosci.

Hansel, D. \& Mato, G. (2003). Asynchronous states and the emergence of synchrony in large networks of interacting excitatory and inhibitory neurons. Neural Comput, 15, 1-56.

Hedstrom, K. L. \& Rasband, M. N. (2006). Intrinsic and extrinsic determinants of ion channel localization in neurons. J Neurochem, 98, 1345-1352.

Hille, B. (2001). Ion Channels of Excitable Membranes. (Sunderland,MA: Sinauer Associates).

Hodgkin, A. L. \& Huxley, A. F. (1952). A quantitative description of membrane current and its application to conduction and excitation in nerve. J Physiol, 117, 500-544. 
Hodgkin, A. L. \& Rushton, W. A. H. (1946). The electrical constants of a crustacean nerve fibre. Proc. R. Soc. Lond. (Biol.), 133, 444-479.

Hopfield, J. J. (1995). Pattern recognition computation using action potential timing for stimulus representation. Nature, 376, 33-36.

Huguenard, J. R., Hamill, O. P., \& Prince, D. A. (1989). Sodium channels in dendrites of rat cortical pyramidal neurons. Proc Natl Acad Sci U S A, 86, 2473-2477.

Inda, M. C., DeFelipe, J., \& Mu?oz, A. (2006). Voltage-gated ion channels in the axon initial segment of human cortical pyramidal cells and their relationship with chandelier cells. Proc Natl Acad Sci U S A, 103, 2920-2925.

Jack, J., Noble, D., \& Tsien, R. W. (1975). Electrical Current Flow in Excitable Cells. (Oxford: Clarendon Press).

Jolivet, R., Kobayashi, R., Rauch, A., Naud, R., Shinomoto, S., \& Gerstner, W. (2008). A benchmark test for a quantitative assessment of simple neuron models. J Neurosci Methods, 169, 417-424.

Knight, B. W. (1972a). Dynamics of encoding in a population of neurons. J Gen Physiol, 59, 734-766.

Knight, B. W. (1972b). The relationship between the firing rate of a single neuron and the level of activity in a population of neurons. experimental evidence for resonant enhancement in the population response. J Gen Physiol, 59, 767-778.

Koch, C. \& Segev, I. (1998). (eds.) Methods in Neuronal Modeling: From Ions to Networks. (MA: The MIT Press).

Kole, M. H., Ilschner, S. U., Kampa, B. M., Williams, S. R., Ruben, P. C., \& Stuart, G. J. (2008). Action potential generation requires a high sodium channel density in the axon initial segment. Nat Neurosci, 11, 178-186.

Kole, M. H. P., Letzkus, J. J., \& Stuart, G. J. (2007). Axon initial segment kv1 channels control axonal action potential waveform and synaptic efficacy. Neuron, 55, 633-647.

Köndgen, H., Geisler, C., Fusi, S., Wang, X. J., Lüscher, H. R., \& Giugliano, M. (2008). The dynamical response properties of neocortical neurons to temporally modulated noisy inputs in vitro. Cereb Cortex.

Lampl, I., Reichova, I., \& Ferster, D. (1999). Synchronous membrane potential fluctuations in neurons of the cat visual cortex. Neuron, 22, 361-374. 
Magee, J. C. \& Carruth, M. (1999). Dendritic voltage-gated ion channels regulate the action potential firing mode of hippocampal ca1 pyramidal neurons. J Neurophysiol, 82, 1895-1901.

Mainen, Z. F., Joerges, J., Huguenard, J. R., \& Sejnowski, T. J. (1995). A model of spike initiation in neocortical pyramidal neurons. Neuron, 15, 1427-1439.

Mainen, Z. F. \& Sejnowski, T. J. (1996). Influence of dendritic structure on firing pattern in model neocortical neurons. Nature, 382, 363-366.

Marx, S. O., Gaburjakova, J., Gaburjakova, M., Henrikson, C., Ondrias, K., \& Marks, A. R. (2001). Coupled gating between cardiac calcium release channels (ryanodine receptors). Circ. Res., 88, 1151-1158.

Marx, S. O., Ondrias, K., \& Marks, A. R. (1998). Coupled gating between individual skeletal muscle ca2 + release channels (ryanodine receptors). Science, 281, 818-821.

McCormick, D. A., Shu, Y., \& Yu, Y. (2007). Neurophysiology: Hodgkin and huxley model-still standing? Nature, 445, 1-2.

Meeks, J. P., Jiang, X., \& Mennerick, S. (2005). Action potential fidelity during normal and epileptiform activity in paired soma-axon recordings from rat hippocampus. J Physiol, 566, 425-441.

Meeks, J. P. \& Mennerick, S. (2007). Action potential initiation and propagation in ca3 pyramidal axons. J Neurophysiol, 97, 3460-3472.

Molina, M., Barrera, F., Fernandez, A., Poveda, J., Renart, M., Encinar, J., Riquelme, G., \& Gonzalez-Ros, J. M. (2006). Clustering and coupled gating modulate the activity in KcsA, a potassium channel model. J. Biol. Chem., $281,18837-18848$.

Naundorf, B., Geisel, T., \& Wolf, F. (2005a). Action potential onset dynamics and the response speed of neuronal populations. J Comput Neurosci, 18, 297309.

Naundorf, B., Geisel, T., \& Wolf, F. (2005b). Dynamical response properties of a canonical model for type-i membranes. Neurocomputing, 20, 421-428.

Naundorf, B., Wolf, F., \& Volgushev, M. (2006). Unique features of action potential initiation in cortical neurons. Nature, 440, 1060-1063.

Naundorf, B., Wolf, F., \& Volgushev, M. (2007). Neurophysiology: Hodgkin and huxley model - still standing? (reply). Nature, 445, E2-E3. 
Palmer, L. M. \& Stuart, G. J. (2006). Site of action potential initiation in layer 5 pyramidal neurons. J Neurosci, 26, 1854-1863.

Post, J. A., Leunissen-Bijvelt, J., Ruigrok, T. J., \& Verkleij, A. J. (1985). Ultrastructural changes of sarcolemma and mitochondria in the isolated rabbit heart during ischemia and reperfusion. Biochim Biophys Acta, 845, 119-123.

Rall, W. (1977). Core conductor theory and cable properties of neurons. In Kandel, E.R., J.M. Brookhardt, and V.M. Mountcastle eds. Handbook of physiology, cellular biology of neurons. (Bethesda, MD: American Physiology Society).

Rauch, A., La Camera, G., Luscher, H. R., Senn, W., \& Fusi, S. (2003). Neocortical pyramidal cells respond as integrate-and-fire neurons to in vivo-like input currents. J Neurophysiol, 90, 1598-1612.

Saito, A., Inui, M., Radermacher, M., Frank, J., \& Fleischer, S. (1988). Ultrastructure of the calcium release channel of sarcoplasmic reticulum. J Cell Biol, $107,211-219$.

Schindler, H. (1984). Cooperativity between acetylcholine-receptor channels. Prog Clin Biol Res, 164, 291-294.

Shu, Y., Hasenstaub, A., Duque, A., Yu, Y., \& McCormick, D. A. (2006). Modulation of intracortical synaptic potentials by presynaptic somatic membrane potential. Nature, 441, 761-765.

Silberberg, G., Bethge, M., Markram, H., Pawelzik, K., \& Tsodyks, M. (2004). Dynamics of population rate codes in ensembles of neocortical neurons. J Neurophysiol, 91, 704-709.

Steinmetz, P. N., Roy, A., Fitzgerald, P. J., Hsiao, S. S., Johnson, K. O., \& Niebur, E. (2000). Attention modulates synchronized neuronal firing in primate somatosensory cortex. Nature, 404, 187-190.

Steriade, M. (2001). Impact of network activities on neuronal properties in corticothalamic systems. J Neurophysiol, 86, 1-39.

Stuart, G., Schiller, J., \& Sakmann, B. (1997). Action potential initiation and propagation in rat neocortical pyramidal neurons. J Physiol, 505, 617-632.

Stuart, G. J. \& Sakmann, B. (1994). Active propagation of somatic action potentials into neocortical pyramidal cell dendrites. Nature, 367, 69-72.

Thorpe, S., Fize, D., \& Marlot, C. (1996). Speed of processing in the human visual system. Nature, 381, 520-522. 
Undrovinas, A., Fleidervish, I., \& Makielski, J. (1992). Inward sodium current at resting potentials in single cardiac myocytes induced by the ischemic metabolite lysophosphatidylcholine. Circ. Res., 71, 1231-1241.

Ursell, T., Huang, K. C., Peterson, E., \& Phillips, R. (2007). Cooperative gating and spatial organization of membrane proteins through elastic interactions. PLoS Comput Biol, 3, e81.

Volgushev, M., Malyshev, A., Balaban, P., Chistiakova, M., Volgushev, S., \& Wolf, F. (2008). Onset dynamics of action potentials in rat neocortical neurons and identified snail neurons: quantification of the difference. PLoS ONE, 3.

Wang, X. J. \& Buzsáki, G. (1996). Gamma oscillation by synaptic inhibition in a hippocampal interneuronal network model. J Neurosci, 16, 6402-6413.

Williams, S. R. \& Stuart, G. J. (2000). Backpropagation of physiological spike trains in neocortical pyramidal neurons: implications for temporal coding in dendrites. J Neurosci, 20, 8238-8246.

Yu, Y., Shu, Y., \& McCormick, D. A. (2008). Cortical action potential backpropagation explains spike threshold variability and rapid-onset kinetics. J Neurosci, 28, 7260-7272. 


\section{Summary}

Studies of pattern formation in many physical systems have identified many tools of nonlinear dynamical systems theory that appear suitable for the investigation of spatio-temporal patterns in neuroscience. In this thesis we apply these tools to study pattern formation in the cerebral cortex on two different scales: the development of spatially complex visual maps on the level of cortical columns, and the onset dynamics of action potential generation on the level of single neurons.

In the first part of this thesis, we develop a quantitatively controlled numerical method to study the dynamical processes resulting from competitive Hebbian learning in the development of orientation preference maps (OPMs). Kohonen's self-organization feature mapping has been widely used to simulate the emergence and arrangement of different feature maps in the visual cortex. Even though the formation of OPMs in this model is known to be affected by an unstable dynamics that removes biologically important structures called spontaneous pinwheel annihilation, the problem of pinwheel stability has been left unexplored in almost all studies of this model and the model's attractor states have largely remained un-characterized.

Our study identifies different dynamical regimes in the Kohonen model and performs a comprehensive study for various system sizes, feature space dimensionalities and stimulus distributions. We first consider the development of OPMs coupled only with retinotopy in a $2 \mathrm{D}+2 \mathrm{D}$ model and characterize the unstable pinwheel dynamics by quantitative analysis of the kinetics of pinwheel annihilation. Different from previous simulation work that overlooked this instability and the resulting pattern rearrangement in map dynamics, our study demonstrates a general way to characterize the long term dynamics in large systems.

Including more feature dimensions in this model, we further test whether the pinwheel dynamics may be stabilized by interactions of different feature maps. Intriguingly, we find an essential 2D mapping close to the symmetry breaking threshold, whereas the additional $n-2$ feature dimensions are suppressed until subsequent bifurcations. Our study reveals the new phenomena of dimension suppression and pinwheel crystallization in different dynamical regimes. The transformation from an initial pinwheel-rich aperiodic pattern to either pinwheelfree orientation stripes or pinwheel crystals appears to be a robust dynamical phenomenon in developmental models. 
In the second part of this thesis, we study the phase plot dynamics of action potential (AP) initiation in conductance based (CB) neuron models and its impact on population coding properties. Cortical recordings of somatic APs have revealed an abrupt onset in phase plots unexpected from canonical HodgkinHuxley-type models. The fast onset of individual spikes has been hypothesized to result from cooperative sodium channel gating and has been hypothesized to lead to a more reliable population response to high frequency inputs.

In our study, we construct and characterize a new class of CB model implementing the mechanism of channel cooperativity. For a low fraction of strongly cooperative channels, this model reproduces the rapid onset dynamics and biphasic waveforms observed in phase plots of cortical APs. Strong sodium channel cooperativity is found to boost the encoding of fast varying inputs.

To examine an alternative explanation for the rapid onset of somatic APs, namely that it is due to lateral currents from the axon initial segment (AIS), we further characterize AP waveforms in multi-compartment neuron models. In spatial extended neuron models constrained to fit known physiological parameters, phase plots of somatic APs faithfully reveal the characteristics of the AP generator. Lateral currents are found to have little impact on the onset dynamics of somatic APs except in the models with large passive dendrites and an extremely high $\mathrm{Na}^{+}$channel density at the AIS. Finally, we test the coding properties of these models and find a slightly weaker gain attenuation in the high frequency sensitivity with increasing of $\mathrm{Na}^{+}$density at AIS than is observed in single compartment models.

In summary, we identify two possible mechanisms for modeling AP generation in cortical neurons with a rapid onset and a fast coding property. We point out that neither the cooperative channel models nor the spatially extended neuron models can easily reproduce the coding properties of cortical neurons which appear to exhibit no attenuation in their high frequency response up to $200 \mathrm{~Hz}$. 


\section{Acknowledgements}

My foremost thanks go to my advisor, Prof. Fred Wolf, for guiding my first steps in the beautiful world of theoretical neuroscience and offering me unique opportunities to do research in various fields. His enthusiasm for science and never-ending supply of insightful ideas has been a continual inspiration to me. I would like to thank Prof. Stefan Treue and Prof. Tobias Moser for being my thesis committee members and supporting me with valuable comments and suggestions throughout my study.

I especially thank to Prof. Michael Hörner and Sandra Drube in IMPRS Neuroscience Program for taking thoughtful care of us international students and making my life and study in Göttingen smooth and enjoyable.

I am grateful to all my colleagues in the Department of Nonlinear Dynamics and BCCN Göttingen. Thanks to Prof. Theo Geisel for heading the vigorous department. Special thanks go to Dr. Matthias Kaschube, Dr. Michael Schnabel, Wolfgang Keil and Lars Reichl for knowledgeable thought and inspiring talks on map project, to Tatjana Tchumatchenko, Michael Kreissl and Dr. Wei Wei for stimulating discussions in 'spike club' and everlasting help, to Anna and Georg Martius for good company in the daily life and warmhearted help in everything, to Dr. Andreas Neef, Dr. Raoul-Martin Memmesheimer and Dr. Marc Timme for sharing with me their scientific visions, and to all members of 'lunch club' for wonderful food and funny stories.

I appreciate all the administrative support from Katharina Jeremias, Corinna Trautsch, Regina Wunderlich and Dr. Tobias Niemann. I especially appreciate the technical help in all sorts of computer-related problems from Yorck-Fabian Beensen and Georg Martius. 
I also wish to thank Prof. Siegrid Löwel and Dr. Karl Schmidt for the delicate cat experiments they have shown me and the hard work together. I want to thank Prof. Tobias Moser and Prof. Maxim Volgushev for providing a professional working environment and teaching on electrophysiological recordings in their laboratories, and Prof. Mike Gutnick for scientific communication and general encouragement.

I owe many thanks to a long list of lovely friends in Göttingen for the happy and memorable time together. Besides several names mentioned above, there are still Le, Yunyun, Tsaiwen, Bell, Stephan, Arwed, Jasmin, Amit, Schanila, Henry, Kamila, et.al. from Neuroscience program and my other Chinese friends like Kefei, Jianfeng and Hong. Special thanks go to the members of 'Weekend /Go Club' (Jin, Tzvetomir, Wei and Xuejie), for fantastic ideas, delicious food and interesting games as well as scientific and cultural exchange.

Last but not least, I should thank my parents for their constant supports in the past years. My deepest thanks are reserved to Xiang, who brings me a wonderful new life in many ways, including raising our lovely daughter together. 


\section{Curriculum Vitae}

Forename: Min Address: Bunsenstr. 10

Surname: Huang

Date of Birth: Sep. 19th, $1981 \quad$ Phone: $\quad+49-(0) 17665781252$

Place of Birth : Fujian, P. R. China +86-(0)13621059405

Citizenship: P. R. China Email: min@nld.ds.mpg.de

\section{Scientific Education}

2004- PhD study in Neurosciences,

Max-Planck-Institute for Dynamics \& Self-organization, Göttingen

Advisor: Prof. Dr. Fred Wolf

2003-2004 Master study in Neurosciences,

Max Planck Research School Neuroscience Program, Göttingen

2003 Bachelor's degree in Biological Science,

School of Life Sciences, Peking University, Beijing

1999-2003 Undergraduate study in Biological Science, School of Life Sciences, Peking University, Beijing

\section{Working Experience}

2005-2006 Teaching Assistant on Neural Modeling,

Neuroscience Program of IMPRS, Göttingen

2001-2003 Editor in a college publication, Science worm, Beijing 


\section{Publications}

- Huang, M., Geisel, T., Löwel, S. and Wolf, F. (submitted) Dimension Suppression and Pinwheel Crystallization in Competitive Hebbian Models of Visual Cortical Development.

- Huang, M., Volgushev, M. and Wolf, F. (submitted) Sodium Channel Cooperativity in Action Potential Initiation and Neural Encoding.

\section{Poster Presentations}

1. Huang, M. and Wolf, F. (2005) Dynamics of Orientation Preference Maps in the Kohonen Model.

6th International Summer School/Conference "Let's Face chaos through nonlinear dynamics" in Maribor, Slovenia

2. Huang, M., Geisel, T., Löwel, S. and Wolf, F. (2007) Dynamics of Orientation Preference Maps in the Kohonen Model.

3rd Bernstein Symposium of the Bernstein Center for Computational Neuroscience in Göttingen, Germany

3. Huang, M. and Wolf, F. (2008) How do Axonal Initiation and Propagation Shape the Waveform of Action Potentials?

2008 Sloan-Swartz Summer Meeting for Computational Neuroscience in Princeton, USA. 